Final Report

FHWA/IN/JTRP-2000/8

\title{
A Highway Travel Information System: Forecasting and Publicizing Delays in the Indiana State Highway Network
}

\author{
By \\ Shu-Ling Yu \\ Research Assistant \\ School of Civil Engineering \\ Purdue University \\ Jon D. Fricker \\ Professor \\ School of Civil Engineering \\ Purdue University \\ Joint Highway Research Program \\ Project Number: C-36-54BBB \\ File Number: 3-3-54 \\ SPR-2204
Conducted in Cooperation with the and the
U.S. Department of Transportation
Federal Highway Administration \\ Indiana Department of Transportation
}

The contents of this report reflect the views of the authors, who are responsible for the facts and the accuracy of the data presented herein. The contents do not necessarily reflect the official views or policies of the Indiana Department of Transportation or the Federal Highway Administration at the time of publication. This report does not constitute a standard, specification, or regulation.

\author{
Purdue University \\ West Lafayette, IN 47907 \\ July 2004
}




\section{TECHNICAL Summary}

INDOT Research

Technology Transfer and Project Implementation Information

TRB Subject Code 55: Traffic Flow Capacity and Measurement

Publication No.: FHWA/IN/JTRP-2000/8, SPR-2204

July 2004

Final Report

\section{A Highway Travel Information System: Forecasting and Publicizing Delays in the Indiana State Highway Network}

\section{Introduction}

The Highway Travel Information System (HTIS) is a long-term pre-trip information system providing information about expected events, such as road constructions. Recent research has focused on real-time and short-term pre-trip information system. Real-time and short-term pre-trip information systems are the most beneficial to those drivers already on the road or about to depart. The HTIS can be beneficial to both highway travelers and construction managers of roadway projects. Construction managers can use the HTIS to check the forecasted traffic impacts of a proposed construction project schedule. Travelers who have flexible schedules and would like to do pretrip planning to avoid unnecessary delays can obtain knowledge of traffic conditions in the near future from the HTIS.

An approach called Workzone Delay Equilibrium Estimation (WDEE) is used by the HTIS to predict traffic conditions as construction zones age. Two extreme values of link volumes are calculated based on two scenarios: "no information”, in which no drivers know about a new road capacity reduction, and "complete information", in which all drivers have adequate information about road construction zones. A relationship between link volumes and the age of a project has been hypothesized and applied to the two extreme cases to estimate link volumes during construction periods. Finally, expected delays on links under construction are estimated based on the estimated link volumes.

The objectives of the HTIS are:

- To predict the delays on the major highways in Indiana that are caused by scheduled roadway projects for expected travel levels and patterns;

- To build a user-friendly interface providing individuals and institutions with easy access to this travel information system;

- To provide INDOT with a method to review (and possibly revise) its construction project schedules;

To establish standard procedures to operate, maintain and update the HTIS.

\section{Findings}

The delays in relation to the age of construction are estimated using the approach of Workzone Delay Equilibrium Estimation (WDEE) hypothesized for the HTIS. WDEE involves the following three steps:

1. Analysis for the "no information" scenario:

In the "no information" scenario, the traffic demands on each link with no construction and at the beginning of road construction are treated the same. The "no information" link flows are estimated based on historical flows at the time before the workzone is added, using a simplified traffic volume forecasting technique developed for the HTIS. The travel delays for the "no information" case can be estimated by applying queueing theory using the "no information" link flows as arrival rates and workzone capacities as service rates. 
2. Analysis for the "complete information" scenario:

In the "complete information" scenario, it is assumed that drivers will divert to alternate routes rather than change their departure times to minimize delays. The travel between each O-D pair for a specific time period and day type is treated to be the same for both the "complete information" and "no information" scenarios. To simulate the "complete information" scenario, two analyses are involved - O-D estimation and traffic assignment. O-D estimation generates an O-D table based on the link flows for the "no information" case. Equilibrium traffic assignment is applied to the network with workzone capacities in place, using the O-D table obtained from O-D estimation. The travel delays for the "complete information" case can be estimated by applying queueing theory using the "complete information" link volumes as arrival rates and workzone capacities as service rates.
3. Estimation of delays during the construction period

The delay on a link is related to the traffic flow on the link. In queueing theory, it is assumed that delay occurs on a link when the traffic volume is greater than its capacity. When delay occurs, the delay on a link decreases as the link volume decreases. The volumes at a workzone are expected to decrease as construction proceeds and more drivers become aware of the construction. The traffic patterns during the construction period are determined by analyzing historical traffic data during construction periods. The relationship between the historical data and the age of construction can be applied to the links in workzones to obtain expected link volumes at any time during the construction period. The expected delays on each link can be estimated based on the expected link volumes.

\section{Implementation}

The HTIS is divided into three subsystems for the purpose of meeting the needs of different groups of users. They are the modeling, project scheduling, and web access subsystems. The modeling and project scheduling subsystems are for management purposes and the web access subsystem is for use by the traveling public.

\section{Modeling subsystem}

The modeling subsystem is built using the Excel VBA and the TransCAD GISDK interfaces. The Excel VBA interface implements the simplified traffic volume forecasting technique proposed by HTIS. The TransCAD GISDK interface performs the analysis of the "no information" scenario, the "complete information" scenario, Workzone Delay Equilibrium Estimation (WDEE) during the transition construction period, delay estimation and delay database generation.

\section{Project scheduling subsystem}

The project scheduling subsystem is built using the TransCAD GISDK interface. There are two major tasks for the project scheduling subsystem. The first one is to manage construction schedule databases. The second one is to assist project scheduling personnel in determining scheduling strategies that avoid excessive delays to traffic by providing functions to adjust project starting date, end date, and lane closure strategies in terms of workzone capacities.

\section{Web access subsystem}

The web access subsystem is built on several scripting languages, including Hyper Text Markup Language (HTML), JavaScript, VB-script, and Active Server Pagers (ASP). The JavaScript and VB-script provide an interactive environment. Drivers may input their desired departure time and receive information about expected delays at workzones that are active during their proposed trips. Motorists can use the information to plan their trips up to two months in advance. 


\section{Contact}

For more information:

Prof. Jon D. Fricker

Principal Investigator

School of Civil Engineering

Purdue University

West Lafayette IN 47907

Phone: (765) 494-2205

Fax: (765) 496-1105
Indiana Department of Transportation

Division of Research

1205 Montgomery Street

P.O. Box 2279

West Lafayette, IN 47906

Phone: (765) 463-1521

Fax: (765) 497-1665

\section{Purdue University}

Joint Transportation Research Program

School of Civil Engineering

West Lafayette, IN 47907-1284

Phone: (765) 494-9310

Fax: (765) 496-1105 
TECHNICAL REPORT STANDARD TITLE PAGE

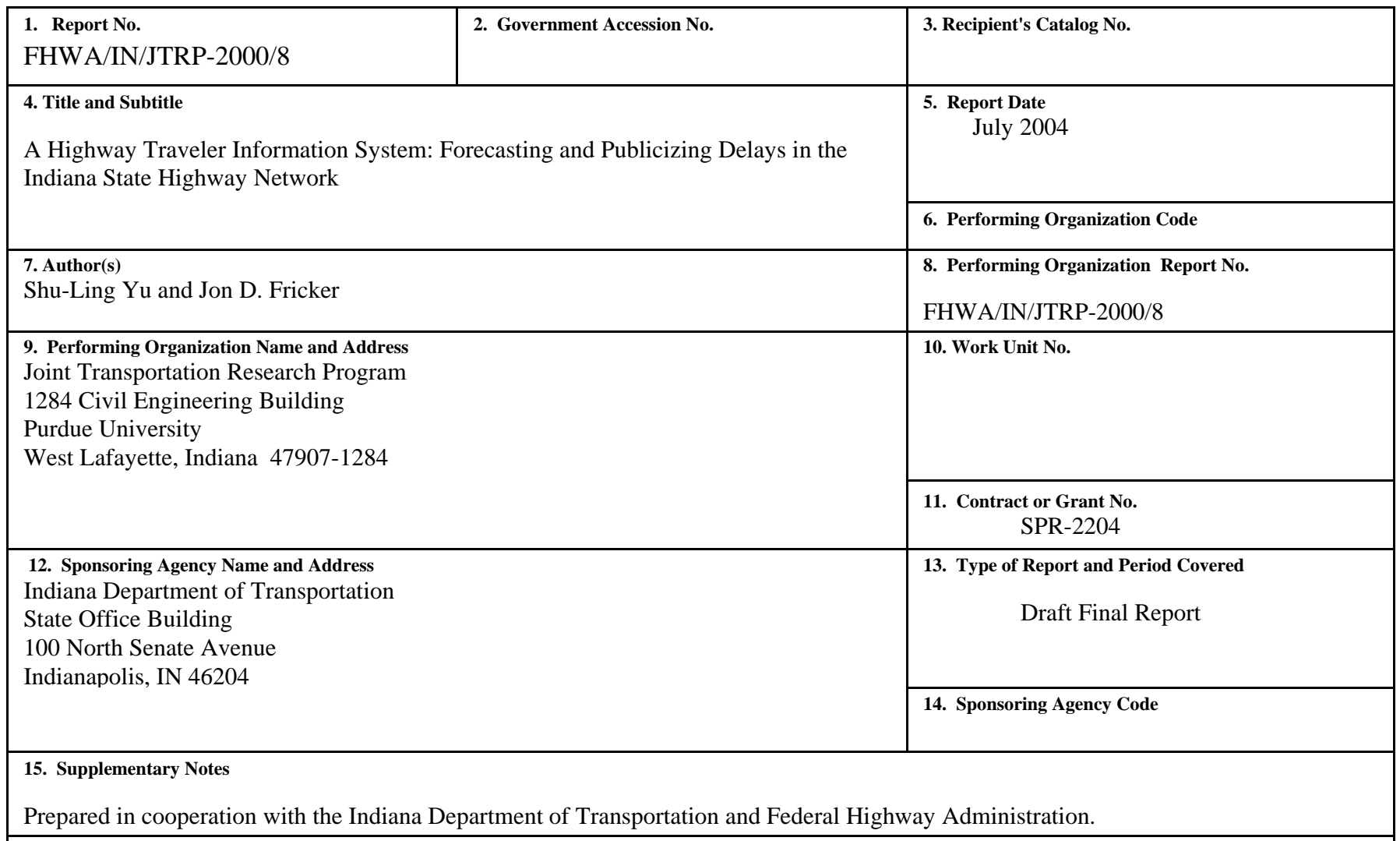

\section{Abstract}

The Highway Travel Information System (HTIS) is a long-term pre-trip information system providing information about expected events, such as road construction. The HTIS can be beneficial to both highway travelers and roadway project schedulers. Project schedulers can use the HTIS to check the forecasted traffic impacts of a proposed construction project schedule. If the schedule would cause unacceptable delays, a scheduler can try new schedules to reduce the delay. Travelers who have flexible schedules and would like to do pre-trip planning can obtain knowledge of traffic conditions in the near future from HTIS to avoid unnecessary delays.

An approach called Workzone Delay Equilibrium Estimation (WDEE) is used by the HTIS to predict traffic conditions as construction zones age. Two extreme values of link volumes are calculated based on two scenarios: "no information", in which no drivers know about a new road capacity reduction, and "complete information", in which all drivers have adequate information about road construction zones. A relationship between link volumes and the age of a construction project has been hypothesized and applied to the two extreme cases to estimate link volumes during construction periods. Finally, the expected delays on links under construction are estimated based on the estimated link volumes.

The HTIS is divided into three subsystems for the purpose of meeting the needs of different groups of users. They are the modeling, project scheduling, and web access subsystems. The modeling and project scheduling subsystems are for management purposes and the web access subsystem is for use by the traveling public. The modeling subsystem is built using Excel VBA and TransCAD GISDK interfaces. The main function of the modeling subsystem is to model traffic conditions and generate a delay database. The project scheduling subsystem is built on the TransCAD GISDK interface. The major tasks for the project scheduling subsystem include schedule database management and assisting project scheduling. The web access subsystem is built on several scripting languages, including Hyper Text Markup Language (HTML), JavaScript, VB-script, and Active Server Pagers (ASP). With the web access subsystem, travelers may input their proposed departure times and receive information about expected delays at workzones that are expected to be active during their trips. Motorists can use the information to plan their trips up to two months in advance.

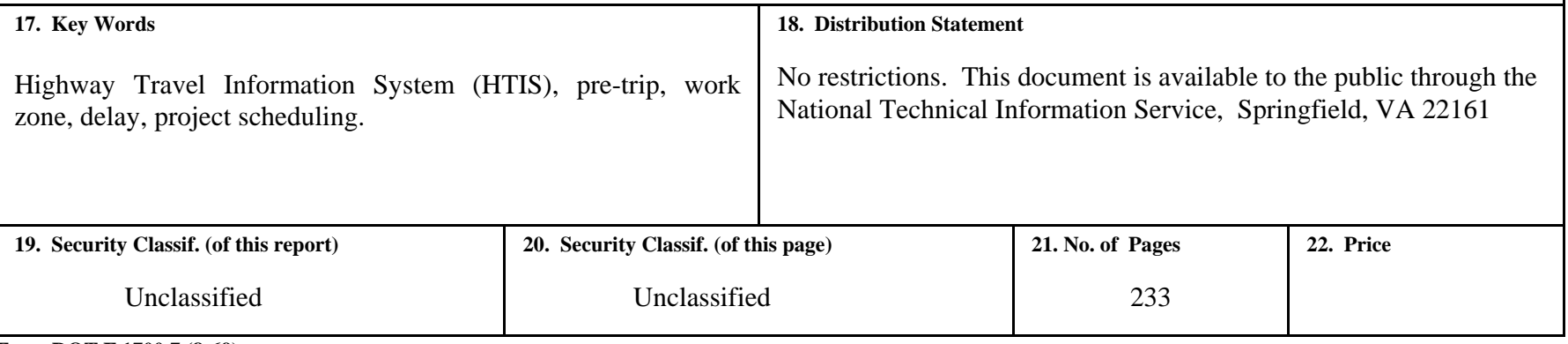




\section{Acknowledgments}

The authors would like to thank the members of the Study Advisory Committee for

their helpful support. These persons are Mike Andrews, Rebecca Black, David Boruff, Jay DuMontelle, Yi Jiang, Roger Manning, Wendall Meyer, Dean Munn, John Nagle, Dan Shamo, Stephen Smith, Mike Wood, and Rick Yunker. Special thanks to Stephen Smith and Dean Munn for providing modeling related reports, assisting collecting data and loaning software; Roy Wasson and Geradine Lampley for providing ATR data; Rick Yunker and John Nagle for furnishing data about construction schedules; David Boruff for helping to find suitable construction projects; Dan Shamo for stimulating new ideas; Richard Lively for clarifying web publishing issues; Yi Jiang for providing reports related to workzone delay; Rebecca Black for assisting on growth factor related issues. 


\section{TABLE OF CONTENTS}

\section{Page}

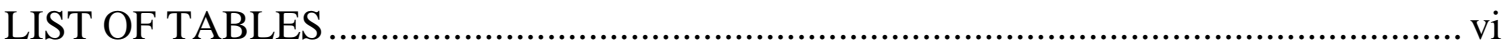

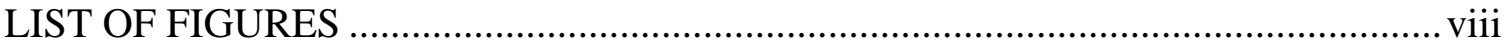

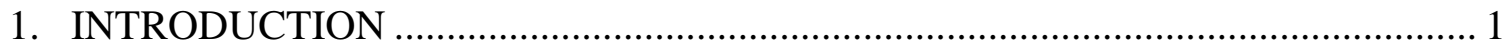

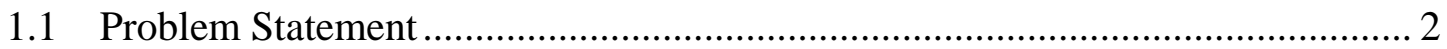

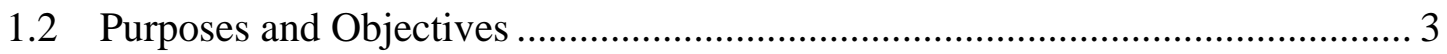

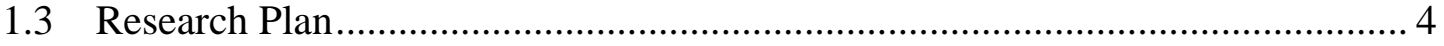

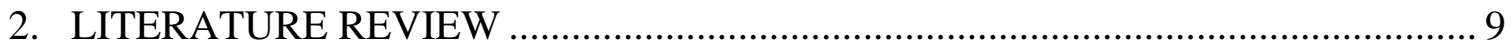

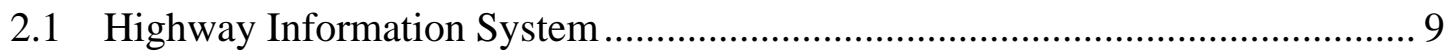

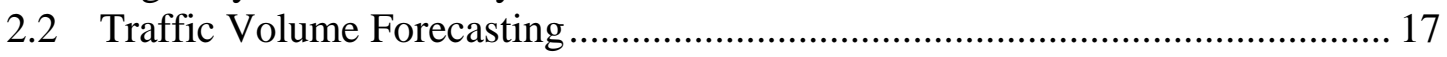

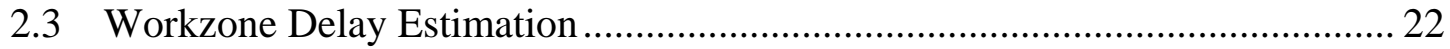

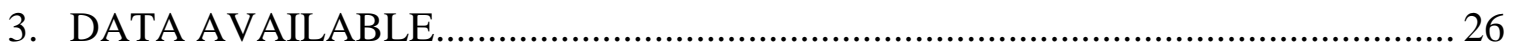

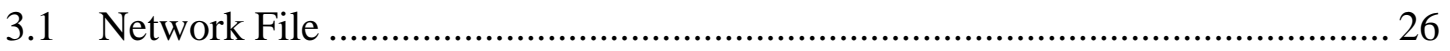

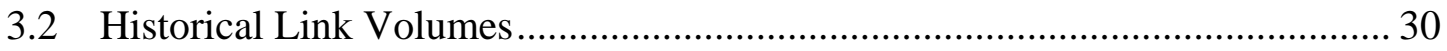

3.2.1 Automated Traffic Recorders (ATRs)....................................................... 30

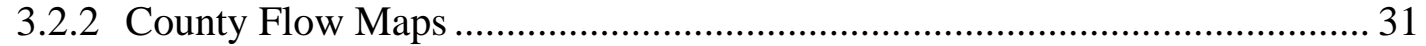

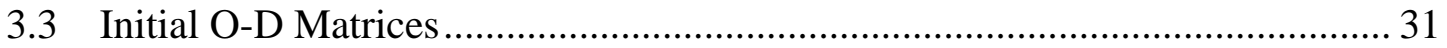

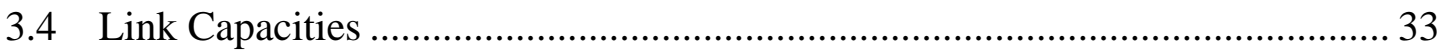

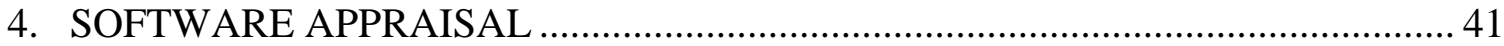

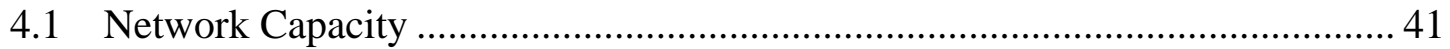

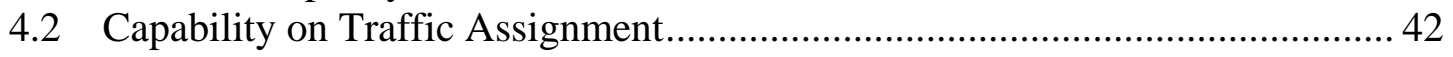

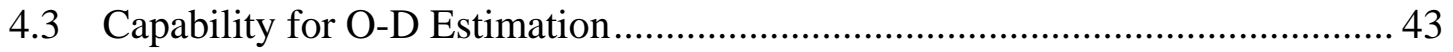

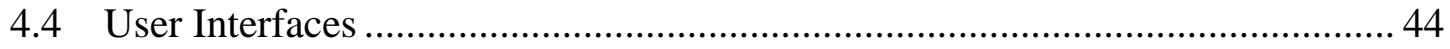

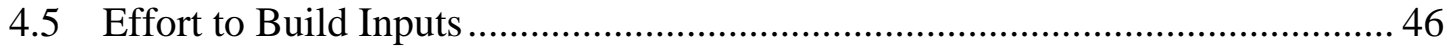

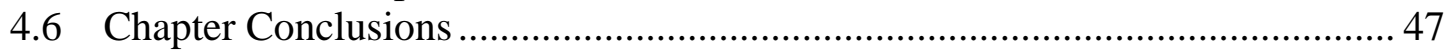




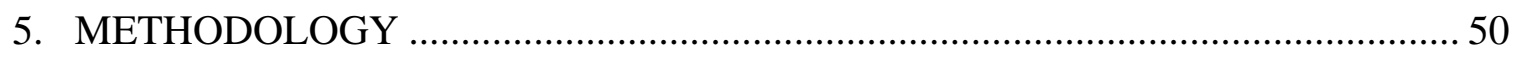

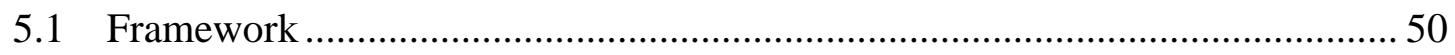

5.2 Traffic Volume Forecasting........................................................................... 55

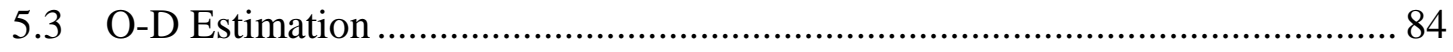

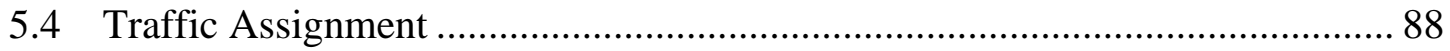

5.5 Delay Estimation........................................................................................ 92

5.5.1 Estimating Upper and Lower Bounds for Delay ......................................... 94

5.5.2 Delay Refinement.......................................................................................... 107

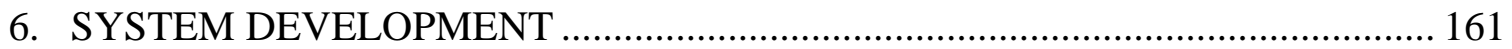

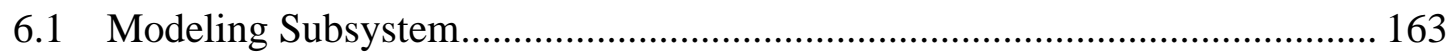

6.2 Project Scheduling Subsystem...................................................................... 169

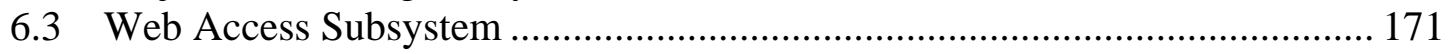

6.4 System Maintenance ....................................................................................... 172

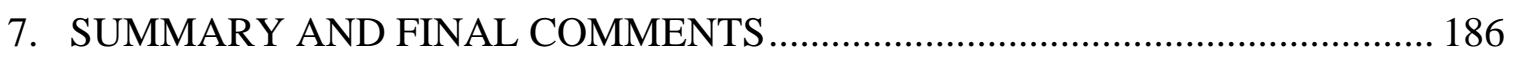

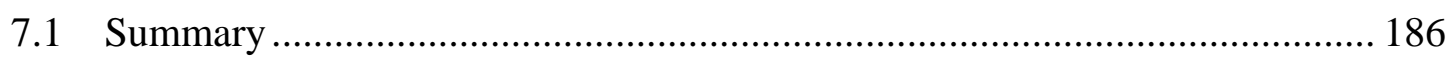

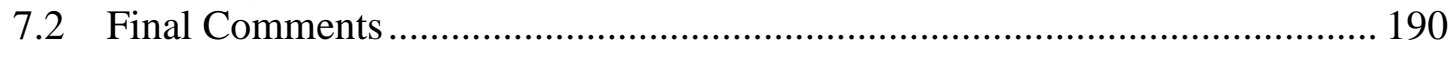

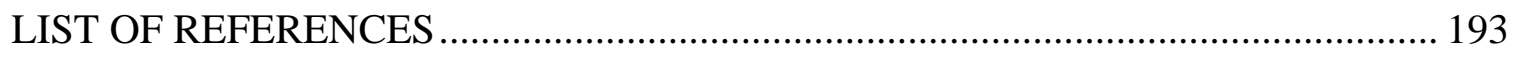

APPENDIX HIGHWAY TRAVEL INFORMATION SYSTEM USER'S GUIDE .... 197

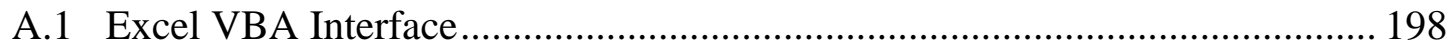

A.2 TransCAD GISDK Interface...................................................................... 203

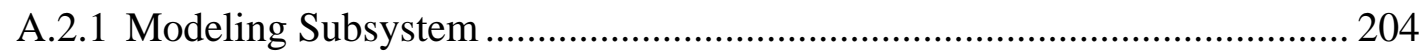

A.2.1.1 Link Volume Forecasting.................................................................. 204

A.2.1.2 Delay Verifications............................................................................... 209

A.2.1.3 Delay Database Generation and Conversion......................................... 210

A.2.2 Project Scheduling Menu ...................................................................... 211

A.3 Web Access Interface ................................................................................... 217 


\section{LIST OF TABLES}

Table

Page

4.1 Comparisons of TRANPLAN and TransCAD as the selected traffic demand software for the highway travel information system

$5.1 r_{s}$ of Spearman Rank Correlation Test on ATR Station 2400 (November $3 \sim$ November 9, 1997).

5.2 48 periods ahead forecasts at base period 160 and 24 periods ahead updated forecasts at base period 184 (November 1997)

5.3(a) Raw data for two ATR stations belonging to the urban interstate highway road class $(11 / 1 / 97 \sim 11 / 7 / 97)$

5.3(b) Raw data for two ATR stations belonging to the urban interstate highway road class $(11 / 1 / 97 \sim 11 / 7 / 97)$.....

5.4 Sum of hourly volumes for both directions and both stations........................... 120

5.5 Peak period definitions and peak period factor calculations ............................. 121

5.6 Peak period definitions based on a single ATR station .................................... 122

5.7(a) Sum of volumes for each direction for days belonging to the same day type...... 123

5.7(b) Sum of volumes for each direction for days belonging to the same day type...... 124

5.8 Larger sum of volumes between two directions for days belonging to the same day type

5.9 Sum of larger sum of volumes between two directions for different day types for all stations belonging to the same road class.

5.10 Directionality calculation 
5.11 Annual growth factors by functional class from 1990 to 1999 in Indiana State .. 128

5.12 Seasonal Adjustment Factors by Functional Class: 1994-1998

5.13 Comparisons of annual growth factors based on the TMG with the ones adopted by INDOT. 130

5.14 Stability analysis on the number of iterations in UE and SUE assignment models

5.15 Workzone capacity formula

5.16 Comparison of measured travel time with calculated values 


\section{LIST OF FIGURES}

Figure $\quad$ Page

1.1 The relationship between link volumes and construction schedules...................... 8

3.1(a) Locations of ATR stations in Indiana (old system) ............................................. 35

3.1(b) Locations of ATR stations in Indiana (new system) ........................................ 36

3.2 Malfunction occurred between November 13 and November 17 in Westbound of ATR Station 1400 in 1997 ........................................................... 37

3.3 Excerpted ADTs in the "ADTVOL" field of a TransCAD network file................ 38

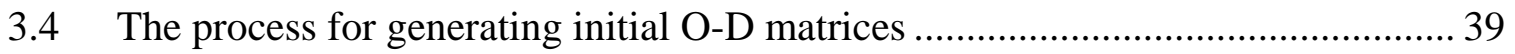

3.5 Example link capacities for levels of service A to E (CapA CapE) in TransCAD network file .................................................................................... 40

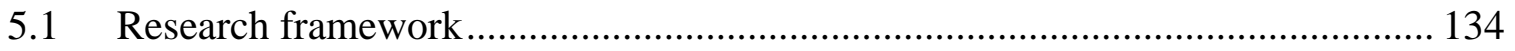

5.2(a) Northbound traffic patterns for normal days at station 2400 (November 1997).. 135

5.2(b) Southbound traffic patterns for normal days at station 2400 (November 1997).. 135

5.3(a) Northbound traffic patterns for the weeks before, during, and after Thanksgiving Holiday at station 2400 (November 1997) ................................. 136

5.3(b) Southbound traffic patterns for the weeks before, during, and after Thanksgiving Holiday at station 2400 (November 1997)

5.4 Northbound traffic patterns for Indy500 car racing event at station 2400 (May 1997)

5.5 The first 160 weekday hourly volumes at ATR station 2400 in November 1997. 
5.6 Autocorrelation functions of the original series ........................................... 140

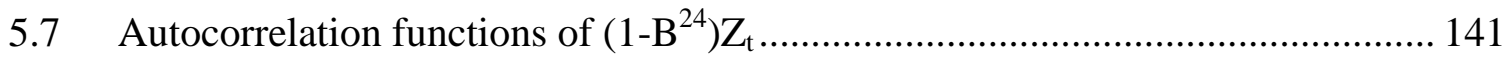

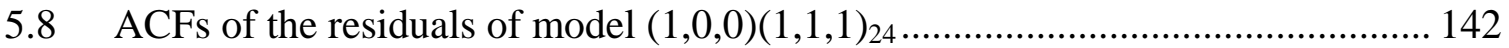

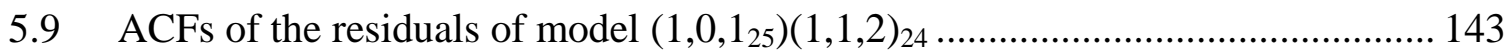

5.10 48-period-ahead forecasts of hourly traffic volumes at base period 160 and 24-periods-ahead updated forecasts of hourly traffic volumes at base period 184 (November 1997)

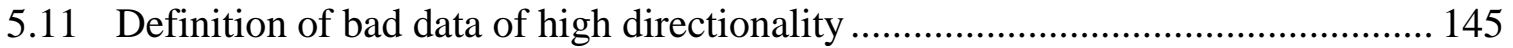

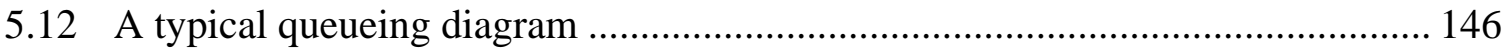

5.13 Probability distribution of actual workzone capacity .................................... 147

5.14 A queueing diagram for situations when the AM-peak period is not followed immediately by the PM-peak period $\left(\mathrm{T}_{\mathrm{b} 1} \leq \mathrm{t}<\mathrm{T}_{\mathrm{e} 1}\right)$

5.15 A queueing diagram for situations when the AM-peak period is not followed immediately by the PM-peak period $\left(\mathrm{T}_{\mathrm{e} 1} \leq \mathrm{t}<\mathrm{T}_{\mathrm{q} 1}\right.$ and $\left.\mathrm{t}+1<\mathrm{T}_{\mathrm{q} 1}\right)$

5.16 A queueing diagram for situations when the AM-peak period is not followed immediately by the PM-peak period $\left(\mathrm{T}_{\mathrm{e} 1} \leq \mathrm{t}<\mathrm{T}_{\mathrm{q} 1}\right.$ and $\left.\mathrm{t}+1 \geq \mathrm{T}_{\mathrm{q} 1}\right)$

5.17 Queueing diagrams for situations when the PM-peak period comes immediately after the AM-peak period

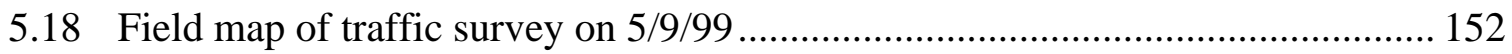

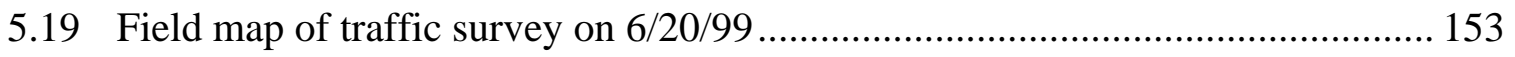

5.20 Queueing diagram for the first survey ...................................................... 154

5.21 Field map of traffic survey on 4/26/2000 through 5/9/2000 .............................. 155

5.22 Comparison of the demands from survey in 2000 and the historical volumes in 1996 156

5.23 Comparison of the demands from survey in 2000 and the historical volumes in 1996 (excluding weekends). 
Figure

5.24 Difference between the demands from survey on the second week and on the first week

5.25 A hypothesized relationship between the volume on workzone link and the age of construction using a logistic function

5.26 Logistic curves for two different percentages of uninformed travelers 160

6.1 Relationships among three subsystems of HTIS 181

6.2 Framework of the modeling subsystem.....

6.3 Flowchart of the Excel VBA interface.

6.4 Flowchart of the modeling subsystem's TransCAD GISDK interface 184

6.5 Time span definitions for traffic assignment.

Appendix Figure

A.1 Relationship of HTIS users’ interfaces 220

A.2 Framework of the Excel VBA interface 221

A.3 Excel VBA introduction menu for the modeling subsystem. 222

A.4 Excel VBA main menu for modeling subsystem. 223

A.5 Open ATR files from the Excel VBA main menu 224

A.6 An example ATR file in federal format (*.fed)............................................ 225

A.7 Special days input menu in the Excel VBA interface ....................................... 226

A.8 Definitions of peak periods for different day types and road classes.................. 227

A.9 The output file of the peak period factors from the Excel VBA interface ........... 228

A.10 The output file of MADTs from the Excel VBA interface ................................ 229

A.11 Framework of the TransCAD GISDK interface............................................ 230

A.12 Screen capture for entering the TransCAD GISDK interface for HTIS ............. 231 
A.13 Main menu of the TransCAD GISDK interface.

A.14 Input menu for TransCAD GISDK interface in modeling subsystem .................. 233

A.15 A map file in TrnsCAD map format (*.map) ……............................................. 234

A.16 An existing hourly volume file in dBase IV format (*.dbf).............................. 235

A.17 An O-D matrix file in TransCAD matrix format (*.mtx)..................................... 236

A.18 Main menu for delay verification for TransCAD GISDK interface in modeling subsystem

A.19 Output of delay verification for upper bound analysis only 238

A.20 The output of delay verification for both upper bound and lower bound analyses

A.21 The dialog box for inputting the file name for delay database. 240

A.22 Dialog box for creating a new Access database

A.23 Dialog box for importing a dBaseIV database 242

A.24 An example delay database 243

A.25 Input menu for TransCAD GISDK interface in project scheduling subsystem ... 244

A.26 Schedule management menu for TransCAD GISDK interface in project scheduling subsystem.

A.27 The existing records in the scheduling database are shown in a new table and on the map ......

A.28 Scheduling evaluation menu in project scheduling subsystem 247

A.29 The total delay of a specific period of time ........................................................ 248

A.30 Framework of the web access interface ……………………............................ 249

A.31 Users' interface for travel information access subsystem .................................... 250

A.32 Output screen for travel information access subsystem ........................................ 251 


\section{INTRODUCTION}

As traffic congestion worsens (The CQ Researcher, 1999), many highway users are eager for information to plan and adjust their trips to avoid unnecessary delays. To improve traffic conditions, advanced traveler information systems (ATIS) are being developed using different methodologies. ATIS focus mainly on providing real-time information such as incident-induced delays. Real-time information systems are the most beneficial to those travelers on the road. For travelers who have not yet started their trips, have flexible schedules and can change their departure times, knowledge of traffic conditions in the near future would be valuable. The travelers could avoid unnecessary delays by scheduling trips departures at non-congested times or by planning beforehand to travel on routes with less or no predicted delays. Therefore, this research proposes a highway travel information system (HTIS) for long-term pre-trip purposes, permitting highway users to improve their travel time, and assisting road construction managers in revising schedules to avoid (if possible) restricted capacity during high traffic demand periods.

Travel information can be presented in a variety of formats. Some information, such as scheduled construction, scheduled events, levels of congestion, detour information, and weather conditions, may be provided either during a trip or before departure, while others such as incidents can be provided only during the trip. 
The time at which information is provided affects the decisions of both highway users and road construction managers. Highway users may use information about scheduled events to determine their departure time and use real-time information to decide to whether reroute while driving. Construction managers, on the other hand, require information about forecasted high demands of traffic to schedule construction projects in order to avoid causing additional delays.

The effectiveness of a travel information system depends on its accuracy, ease of use and ease of maintenance. Accuracy and ease of use can win the trust of the system users. Ease of maintenance makes the information system maintainers' jobs easier and prolongs the life of the system. It is the goal of the system developers to build a travel information system with all of the above features.

Therefore, the main purpose of the HTIS is to establish an accurate, user-friendly and easy-to-maintain pre-trip travel information system to meet the needs of highway users, construction managers and system maintainers.

\subsection{Problem Statements}

Some problems may confront several parties in the absence of an appropriate travel information system.

\section{A. Highway users}

Excessive delay may occur if drivers have incomplete information about scheduled or existing reductions in roadway capacity. Drivers can benefit from information provided during their trips. They can also plan and adjust their trips if relevant information is provided before a trip is made. Drivers may use real-time information to avoid a traffic 
jam, or use information about scheduled events before their departures to avoid the most congested periods.

B. Road construction managers

Sometimes, scheduled construction coincides with periods of high traffic demand. Highway construction is one of the major causes of delay to traffic flow. If construction managers can reschedule projects to avoid seasons, days, or hours of high traffic demand, the negative impacts of highway construction on traffic flow can be reduced. If delay costs to society are compared against the possible increases in construction costs, the rescheduling of projects may be justified.

\subsection{Purposes and Objectives}

One of the problems that the maintainers of some travel information systems have is how to estimate the diversion rate from a route with road construction to alternate routes. The diversion rate depends on many factors, such as the amount and nature of travel information understood by the driver, the traffic conditions on major routes relative to those on alternate routes, drivers' preferences and weather conditions. For example, some drivers may choose to divert on sunny days while stay on the original routes on snowy days. Among these factors, the amount and nature of travel information understood by the driver have the greatest influence on the diversion rate. Diversion rates vary as construction projects go on. At the beginning of a road project, few drivers may have adequate information with which to choose alternate routes. The diversion rates may be small at first. As the road construction continues, more drivers become aware of the capacity reductions, and consider alternate routes. The diversion rates 
become larger. Because diversion rates have this dynamic characteristic, the actual volumes on the original routes become hard to predict.

The dynamic characteristic of diversion rates as construction projects go on is addressed in this research. Once this dynamic characteristic of diversion rates can be successfully addressed, the delays caused by scheduled roadway projects can be predicted. With the delay information, highway users may schedule their trips to avoid unnecessary delays. Project schedulers may use the delay information to evaluate the impacts on construction projects on traffic and make necessary adjustments.

The objectives of this research are listed as follows:

(1) To predict the delays on the major highways in Indiana State caused by scheduled roadway projects for expected travel levels and patterns;

(2) To build a user-friendly interface providing individuals and institutions with easy access to this travel information system;

(3) To provide INDOT with a method to review (and possibly revise) its construction project schedules;

(4) To establish standard procedures for information updates using the newest available data.

\section{$\underline{1.3 \text { Research Plan }}$}

This research introduces an approach called Workzone Delay Equilibrium Estimation (WDEE) to predict traffic conditions as construction ages. WDEE takes into consideration that a certain percentage of uniformed travelers may not make informed decisions, and fins a new "steady state" when the existence of the workzone has become 
widely known. By applying a hypothetical relationship between link volumes and the age of construction, the link volume at any time during the period before the system reaches a new "steady state" can be estimated.

The flow rate entering a workzone can fall between two extreme values. These extreme values correspond to two scenarios:

- The "no information"scenario, in which no drivers know about a new road capacity reduction,

- The "complete information" scenario, in which all drivers have adequate information about road construction zones.

To simulate the "no information" scenario, the link flows are estimated based on historical flows at the time before the workzone is added. The link flows for the "no information" case are assumed to be the same as that for a diversion rate of zero. The travel delays for the "no information" case can be estimated based on the link flows for the "no information" case and workzone capacities. To simulate the "complete information" scenario, two analyses are involved, O-D estimation and traffic assignment. O-D estimation is applied to generate an O-D table based on the link flows for the "no information" case. Equilibrium traffic assignment is applied to the network with workzone capacities in place based on the O-D table obtained from O-D estimation. Neither scenarios will be strictly true, but they form the upper and lower bounds on travel delay during the road construction period.

A relationship between link volumes and construction schedules is hypothesized and applied to estimate link volumes during construction periods (Figure 1.1). As road construction proceeds, diversion rates and link volumes gradually shift from the results of 
the "no information" scenario toward the results of the "complete information" scenario. The link volume for any time during construction periods can be estimated by applying the hypothetical relationship to the two extreme values of link volumes.

Finally, the expected delays on each link are estimated based on the estimated link volumes. With WDEE, the expected delays are linked to construction schedules. The changes of the link volumes related to construction schedules can therefore be addressed without having to predict diversion rates from the main route.

The major tasks of this research include:

(1) Collect and process historical traffic data: Find the traffic patterns associated with some scheduled events and holidays to forecast the demands on the roadway links within Indiana.

(2) Estimate statewide trip tables: Apply O-D estimation to estimate statewide trip tables from forecasted demands on links within the Indiana State highway network.

(3) Perform traffic assignment: Apply equilibrium traffic assignment to simulate the “complete information” scenario in which all drivers have adequate information about road construction zones.

(4) Estimate delays: Generate delay using a formula based on queueing theory.

(5) Refine delay estimates: Generate rules to depict dynamic changes of traffic conditions due to different levels of driver knowledge about roadway capacity reductions and alternate routes.

(6) Verify and feedback: Conduct traffic surveys to verify the estimated delays. Receive feedback from initial users and system maintainers. Modify the system correspondingly. 
(7) Build user interfaces to disseminate traffic information.

(8) Propose standard procedures for system maintenance. 


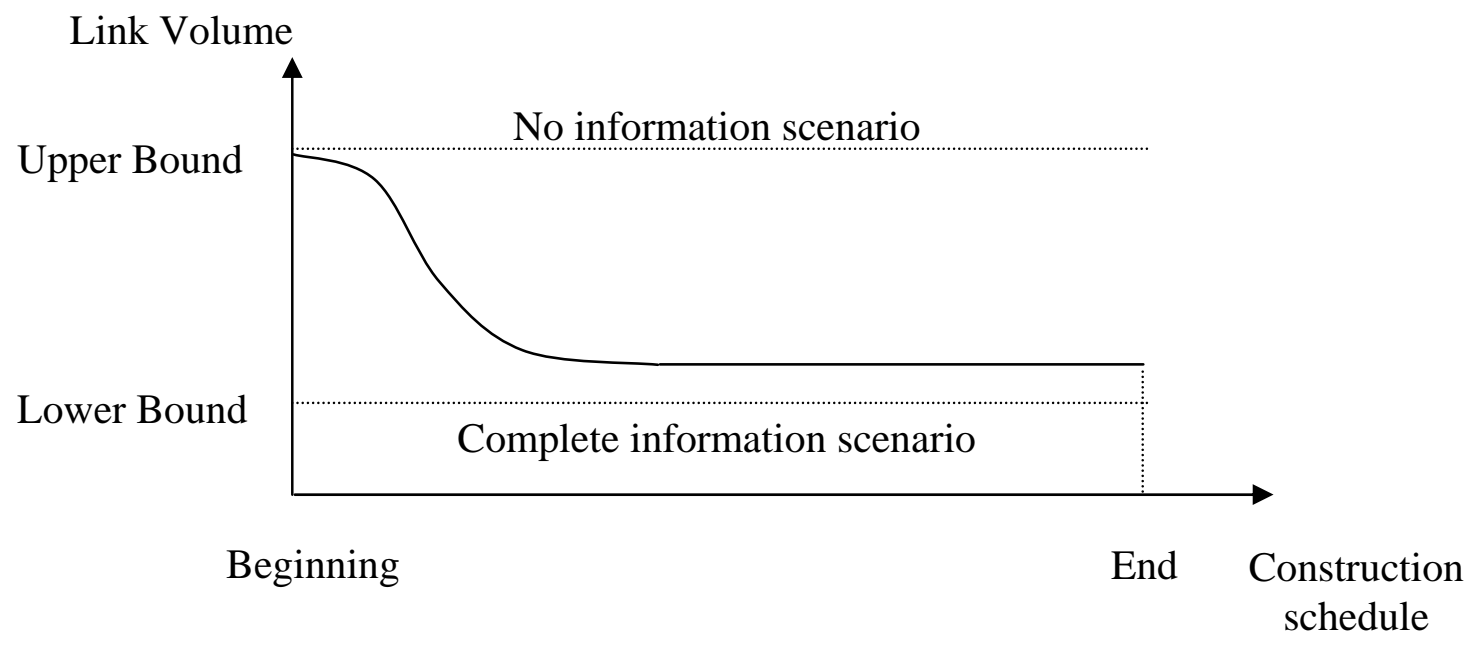

Figure 1.1 The relationship between link volumes and construction schedules 


\section{LITERATURE REVIEW}

This chapter reviews past studies on three important topics related to the HTIS highway information systems, traffic volume forecasting methods and workzone delay estimation. Section 2.1 reviews past studies of highway information systems. Previous research about travel information systems can be categorized into three parts based on the time at which information is provided - real-time information systems, short-term pretrip information systems, and long-term pre-trip information systems. The characteristics and development of these three information systems are summarized.

Section 2.2 presents several traffic volume forecasting methods proposed in the past. Not only various models and techniques applied to traffic forecast are reviewed, the difficulties of forecasting traffic volumes in this research are also brought up.

Finally, a review of workzone delay estimation is given in Section 2.3, in which four main approaches used to estimate workzone delay are introduced to readers. Their pros and cons are discussed so that an appropriate approach for the HTIS is suggested.

\subsection{Highway Information System}

The current information systems can be divided into three groups - real-time, shortterm pre-trip and long-term pre-trip -information systems according to the time information is provided. Real-time information systems provide travelers information 
during driving, short-term pre-trip information systems provide information shortly before departure (normally within one day), and long-term pre-trip information systems provide information one day or more before departure. Real-time and short-term pre-trip information systems are the most suitable for disseminating information about unexpected events such as incidents, while long-term pre-trip information systems are more suitable for informing drivers of expected events such as roadway construction. The following discusses related research on real-time and short-term pre-trip information systems, and long-term pre-trip information systems.

A. Real-time and short-term pre-trip information systems

Recent research on real-time and short-term pre-trip information systems has focused on developing advanced traveler information systems (ATIS). ATIS are an integral component of Intelligent Transportation Systems (ITS). The main objective of ATIS is to provide travelers with dynamic route guidance and real-time information via different media, such as in-vehicle route guidance systems, cellular telephones, cable television, information kiosks and the Internet. The evolution of ATIS started from the one-way communication systems, such as variable message signs (VMS) and highway advisory radio (HAR) (Adler and Blue, 1998). In order to provide individual travel assistance, a wide range of new technologies has been applied, such as real-time traffic surveillance and control systems, electronic route guidance systems (ERGS) and in-vehicle route guidance systems (IVRGS). These new technologies enable users to interact with ATIS and provide individualized path search and dynamic route guidance. Despite of the above evolutions, the development of ATIS is still in progress. 
The effects of information provided by ATIS on travelers' behavior have been discussed by several researchers. Because ATIS are not well developed, the assessments of the effects of information on travelers' behavior are based on existing information about dissemination media or simulation results. Polydoropoulou et al. (1994) categorized these studies into two groups - revealed preference approach and stated preference approach.

The revealed preference approach analyzes drivers' behavior in real-life situations on the basis of respondents' reports, such as diary surveys and field observations. The advantage of applying the revealed preference approach is that the resulting data are based on travelers' actual behavior. The disadvantage of this approach is that the costs of collecting data are high. In addition, this approach may not be applied to new technologies that have not been implemented, because no actual data are available. Polydoropoulou et al. (1994) analyzed travelers' behavior under the influence of traffic information based on diary surveys. About 22\% commuters received pre-trip traffic information from radio and 5\% from TV. Sixteen percent of commuters had their route choice influenced by pre-trip information. It was concluded that a more reliable and more frequently updated traffic information system than radio would gain driver confidence and stimulate the acquisition of traffic information.

The stated preference approach obtains drivers' impressions and reactions to hypothetical scenarios through questionnaires, phone interviews and simulators. The advantages of applying the stated preference approaches are that some environmental factors are controllable and the costs of obtaining data are relatively lower than using the revealed preference approach. However, the resulting data may not reflect travelers' 
actual behavior. Additional verification is required. There are a lot of studies using the stated preference approach. Some of them are discussed herein. According to Barfield et al.'s (1991) study about the influence of information from radio, TV, variable message sign (VMS) and phone on Seattle commuters' behavior, about one third of commuters would check information via TV before driving and over $60 \%$ of commuters would check information via commercial radio stations before and during driving. Over $50 \%$ of commuters have changed departure times or switched to alternate routes based on traffic information. Only a small percentage of commuters have changed modes based on traffic information. Khattak et al. (1995) evaluated the influence of radio traffic reports (RTR) on Chicago commuters. Over $65 \%$ of commuters use RTR during driving and $40 \%$ use RTR before driving. About $60 \%$ of commuters have changed departure times or switched to alternate routes based on RTR. Khattak et al. (1999) conducted phone interviews about the influence of information via radio, TV, phone on the behavior of residents in San Francisco Bay Area. About 48\% of residents listen to radio before departure and 26\% watch TV before departure. Eighty-two percent of automobile noncommuters, $68 \%$ of automobile commuters, $66 \%$ of transit commuters, and $48 \%$ of transit noncommuters are likely to adjust trips in response to travel information. Allen et al. (1991) applied a self-designed simulator to analyze the responses of sampled drivers to four congestion scenarios (11-minute delay - long-trip, 18-minute delay - long-trip, 30-minute delay - long-trip, and 30-minute delay - short-trip) and five in-vehicle navigation systems (none, static map, dynamic map, advanced map and route guidance). The results show that drivers diverted much earlier for both 30-minute delay conditions. 
In addition, the more sophisticated navigation systems are, the higher the diversion percentages are.

As can be seen from the studies with either the revealed preference or the stated preference approaches, the effects of information on the choices regarding route and departure times for each traveler are quite different. There are only a few models built to describe the system performance resulting from the aggregation of individual behavior. Arnott et al. (1991) proposed a simplified model for describing system performance due to incidents on a two-route case. The effects of incidents were simulated by treating link capacity as a stochastic variable. The probability of low capacity on a particular route represents the significance of the incident that occurred on that route. The system performances were divided into three scenarios based on the level of conveying information - zero information, partial information and full information. With zero information, travelers learn nothing about road capacities. Travelers' choices of routes and departure times are based on rational expectations and past experience. With full information, travelers learn all about road capacities early enough for them to adjust their routes and departure times, so that an equilibrium status can be reached. In equilibrium, no one can reduce travel costs by changing route or departure time. It was concluded that the expected costs per driver in the partial information scenario may be higher than in the no information scenario due to the effect of "concentration", which results from too many drivers (but not all of them) following the route guidance.

The concept of the zero information, partial information and full information scenarios in Arnott et al.'s model is the same as in the HTIS. The differences between their model and the HTIS include: 
(1) The stochastic link capacities in their model are for simulating the effects of incidents. It is the effects of workzones that are the focus of the HTIS. Usually the workzone capacities are treated as deterministic;

(2) Their model considered at most two links, while the HTIS addresses the entire road network of Indiana;

(3) The effect of "concentration" happens only when too many travelers (but not all of them) follow the same route, which is suggested by a route guidance system. The HTIS deals with workzone information instead of recommending a specific route for travelers to follow. Therefore, the effect of "concentration" is not discussed in the HTIS.

The effects of information disseminated via the World Wide Web (WWW) on traveler behavior regarding route choices and departure time choices are yet to be decided. The WWW has been widely used for disseminating different kinds of information, such as news, weather and advertising. The formats of information that can be disseminated via the web range form pure text and images to audio and video clips. Several scripting languages such as Javascript, VB-script, Common Gateway Interface (CGI) and Microsoft Active Server Pages (ASP) enable the WWW to interact with users and provide personalized information according to users' preferences. An example of a multimedia-based highway information system (MMHIS) in geographic information system (GIS) shell was shown in Wang and Elliott's study (1996). The MMHIS has a feature of dynamic displaying data with video frames upon a user’s request by clicking on a location on the map. As computer devices continue to improve and the prices of these devices continue to drop, the number of Internet users in the nation has reached 106 
million, over $52 \%$ of American adults (Sefton, 2000). In considering the WWW soon to become one of the major media, the HTIS includes it as one of the media for disseminating travel information.

B. Long-term pre-trip information systems

Little attention has been given to long-term pre-trip information systems. Recent research has focused on the real-time and short-term pre-trip information systems. The real-time and short-term pre-trip information systems, however, provide only information at the time it is accessed, in order to meet the requirements of disseminating information about unexpected events. The long-term pre-trip information systems, on the other hand, can provide motorists with knowledge of traffic conditions about expected or planned events several months in advance, depending on the characteristics of those events. Travelers who are planning ahead and have flexible schedules may be able to reschedule their trips. Drivers in traffic may adjust their routes according to the real-time information to minimize expected delay. Drivers about to begin a trip who consult short-term pre-trip information system may be able to reschedule their trips or modify their routes.

Previous studies on the effects of road construction (one of the most common planned events) on traffic have diverted much attention toward the short-term effects resulting from different traffic control strategies. Nemeth and Rouphail (1982) studied the merging behavior in response to traffic control devices using the self-built microscopic simulation model Freeway Construction (FREECON). Dudek et al. (1986) conducted surveys on lane closures on 4-lane highways in Dallas, Texas and Oklahoma City, Oklahoma, and studied the relationships between hourly volumes and user costs induced by workzones. Zhang et al. (1989) applied the simulation model FREQ10PC to assess 
the different effects of traffic control strategies on traffic at the Oakland Bay Bridge in San Francisco, California, and the impacts of nighttime lane closures on the traffic on I80 northeast of the Oakland Bay Bridge. Robertson, Palumbo and Rice (1995) evaluated the impacts of a workzone at Metropolitan Boulevard in Montreal on adjacent roads. Boruff (1994) evaluated the impacts of the I70 reconstruction from I465 to the Belmont Avenue overpass in Indianapolis, Indiana by calculating the percentage changes of total traffic volumes on both the major route and the alternate route.

In the studies mentioned above, none explores day-to-day variations in traffic patterns due to workzones during the transition time before an equilibrium state is reached. The day-to-day variations in traffic patterns due to workzones were evaluated in Jha and Sinha's study (1996). Jha and Sinha applied a dynamic traffic simulator (DYNASMART) to a test network with 10 zones, 50 nodes and 168 links, and simulated the effects of different percentages of travelers who trust and follow the route guidance on the system performance in terms of average travel time. Their study can be a good starting point for studying the effects of long-term pre-trip information on traffic. Two aspects in their study need to be further developed:

(1) The lane closure strategies considered are only full lane closures (closures of all lanes on links under construction), which are not common for state highways or higher. The most common lane closure strategies for Indiana highways are partial lane closures, in which the traffic flow is maintained.

(2) The network used in their study is only a test network. The implementation of DYNASMART on long-term events in real networks of a large scale such as the Indiana network can be difficult, because complicated modeling skills are required. 
The HTIS further explores day-to-day variations in traffic patterns due to workzones during the transition time before an equilibrium state is reached in two ways:

(1) Consider both full lane closure and partial lane closure strategies.

(2) Verify the impacts of workzones on traffic using a real network.

\subsection{Traffic Volume Forecasting}

Previous research on traffic volume forecasting applied various techniques. Some of the studies applied neural network models (Dougherty and Cobbett, 1997; and Yun et al., 1998), while some used time series models (Voort, Dougherty and Watson, 1996) to forecast traffic volumes. The comparisons of these two models with several other techniques are shown in Kirby et al.’s study (1997) and Smith and Demetsky’s study (1997).

Kirby et al. (1997) evaluated the accuracies of using upstream volumes to predict downstream volumes based on three forecasting models - neural network models, time series models and the ATHENA model, which is a short-term forecasting model developed by Institut National pour la Recherche sur les Transports et leur Sécurité (INRETS). The data at three stations located about 30 kilometers upstream from the target station were used to forecast the volumes of the target station. It was concluded that both the neural network and the time series models can get a good forecasting result. The ATHENA model was evaluated to be better than both the neural network and the time series models.

Smith and Demetsky (1997) reviewed the accuracies of forecasting results using four models - historical average, time series, neural network and nonparametric regression 
models. They also accessed the transferability of these models. The data used for forecasting were two sets of historical data from two locations. The first set of data was used to evaluate the accuracy of the results based on the four models. It was shown that the nonparametric regression model outperforms the other methods. The neural network model was the second, followed by the time series model third and the historical average model last. The time series model was excluded from the evaluations of model transferability because it does not allow missing data, a common situation in practice. The remaining three models were further evaluated for their transferability using the second set of data from a different location. The transferability of the neural network model was evaluated by processing the second set of data without "retraining", which means the parameters generated based on the first set of data are used directly without updates from the second set of data. The forecasting results showed the nonparametric regression model still remains in first place. The results using the neural network model without "retraining" are even worse than using the historical average model. The neural network model was concluded to be not transferable.

The accuracy of the forecasting results from applying the time series model to the data at the same location needs to be evaluated. The evaluation of the time series model conducted in Kirby et al.’s (1997) study was based on the data 30 kilometers upstream from the target location. In Smith and Demetsky’s (1997) study, the time series model was excluded because it was unable to process nonconsecutive data. Although the situation of missing data also occurs to the ATR data due to malfunctions of equipment, it is still not difficult to find adequate ATR data for use of the time series model in this HTIS study. Consequently, the time series model being unable to process 
nonconsecutive data is not a problem for this research. The evaluation of the time series model based on data at an ATR location in Indiana is performed in Section 5.2.

In addition to the accuracy of forecasting results and model transferability, the selection of a suitable traffic volume forecasting technique for the HTIS also depends on the availability of historical data and the time required performing traffic volume forecasting on all links within Indiana.

(1) Availability of historical data: The historical hourly volumes are needed either for use in building forecasting models or for directly forecasting volumes by transferring models from other locations. Forecasting volumes by transferring models from other locations may not need as much historical data as when building models "from scratch.” The forecasting models themselves cannot forecast hourly volumes without using any historical hourly volumes as a basis. Most of the links within the Indiana do not have historical hourly volumes. The only historical data available for all links within Indiana are the average daily traffic volumes (ADTs) on county flow maps. The problem of insufficient historical volume data is explained in Section 3.2. (2) Time required performing traffic volume forecasting on all links within the Indiana: The existing Indiana highway network file in TransCAD format contains over 16,000 links, and the network file in TRANPLAN format contains over 1,100 links. The traffic volume forecasting process needs to be repeated for each link within the network because the demand for each link is required for the O-D estimation process in the "complete information” scenario to generate the demand for each O-D pair. If the time to process a single link is one minute, the time to process all links in the TransCAD network file is over 260 hours, and the time to process all links in the 
TRANPLAN network file is over 18 hours. Therefore, any forecasting technique that requires over hours to process a single link is not practical for use in the HTIS. In considering the above two reasons, advanced traffic volume forecasting models such as nonparametric regression, time series and neural network techniques are not appropriate for use in the HTIS. A simplified approach for traffic volume forecasting is proposed for the HTIS by modifying the approach to adjust 48-hour counts taken on sample sections to AADTs for the same base year in the Traffic Monitoring Guide (TMG) (FHWA, 1995).

The TMG issued by the Federal Highway Administration (FHWA) provided a simplified approach for estimating average adjustment factors for link volumes (monthly factors, day-of-week factors and growth factors) for links of the same group for use in adjusting daily volumes from short-term surveys to AADTs of the same base year. The criterion for grouping links with similar traffic patterns is by classification of links. The TMG suggests five groups - urban interstate highways, other urban highways, rural interstate highways, other rural highways, and recreational highways. It is not the interest of the HTIS to distinguish recreational highways from other highways. Therefore, only the first four groups are used to group the ATR data. The data required for calculating the average adjustment factors for link volumes include two sets of data that are routinely collected: (1) hourly volumes collected from the Automated Traffic Recorders (ATRs), which are loop detectors and weigh-in-motion devices used for collecting continuous traffic data; and (2) the 48-hour counts taken on sample sections. The calculation process involves three steps: (1) grouping ATR data based on road classes of links; (2) calculating average factors for each group based on the ATR data of the same group; (3) 
applying the average factors for each group to the 48-hour counts taken on sample sections, which belong to the same group.

Because the AADTs in the base year 1995 are available in the TransCAD network file, there is no need for AADT conversions. However, the AADTs in the TransCAD network file are required to be converted to AADTs in current year. The average growth factors are calculated following the process suggested in the TMG. The calculation process for average growth factors is implemented in the HTIS (see Section 5.2).

The HTIS proposes a simplified traffic volume forecasting approach by modifying the daily volumes adjustment procedure proposed in the TMG. This simplified approach adopts the same grouping technique as in the TMG. The differences between this approach and the TMG link volumes adjustment procedure are the required data and the calculation process. The data required include the ATR data and the AADTs from the TransCAD network file, which are converted from the data stored in the county flow maps. The calculation process involves five steps:

(1) Grouping ATR data based on road classes of links;

(2) Calculating average growth factors for each group based on the ATR data of the same group;

(3) Applying the growth factors for each group to the AADTs from the TransCAD network file to obtain AADTs of current year;

(4) Calculating average peak period factors and directionality for each group based on the ATR data of the same group; and

(5) Applying the peak period factors and directionality for each group to the AADTs of current year to obtain hourly volumes for different peak periods. 
The detailed calculation processes of this simplified traffic volume forecasting approach and an example are shown in Section 5.2.

One of the advantages of this simplified approach is that it does not require historical hourly volumes for all links. The only required historical data are historical hourly volumes for ATR stations and historical daily volumes for all links stored in the TransCAD network file. Unlike the existing traffic volume forecasting techniques, this approach can be applied to all links, including those without historical hourly volumes. In addition, the processing time of estimating hourly volumes for all links using this simplified approach is over $90 \%$ shorter than using advanced traffic volume forecasting techniques such as time series and neural network techniques.

\section{$\underline{2.3 \text { Workzone Delay Estimation }}$}

Research on the impacts of highway workzones on traffic has used different ways to quantify the effects. The most commonly used quantitative indices to evaluate the impacts of workzones on traffic include, queueing lengths, workzone user costs, workzone delays and total travel time.

Queueing lengths can be estimated in several ways, such as a deterministic queueing approach, stochastic queueing methods, a shock wave approach and a coordinate transformation time-depend technique. Dixon et al. (1998) compared the queueing length estimation techniques of deterministic, shock wave, and coordination transformation with an estimated field queue. Among the three techniques, the coordinate transformation technique can produce the closest values to the estimated field queue. All of the three techniques tend to underestimate the queue length. The use of queue length as an index 
for evaluating the impacts of workzones may be sufficient for choosing better traffic control strategies. However, queue length is not the way most drivers measure traffic congestion; they do it in terms of time. Therefore, providing queue lengths as travel information cannot efficiently assist travelers on trip planning.

The workzone user costs convert the effects of all impacts of workzones into money values. The definitions of user costs at workzones vary in different studies. Memmott and Dudek (1982) defined the total user costs at workzones using seven components: (1) cost of delay, (2) delay cost of going through the workzone at reduced speed, (3) cost of the speed-change cycle, (4) operating cost of the speed-change cycle before a queue is present, (5) additional operating cost of the speed-change cycle when a queue is present, (6) vehicle running cost before a queue is present, and (7) additional vehicle running cost when a queue is present. Soares and Najafi (1998) divided workzone user costs into three components - travel time delay costs, vehicle operating costs, and accident costs. The advantage of using workzone user costs to evaluate the impacts of workzones is that it includes money-related costs in addition to time-related costs. The use of workzone user costs as travel information has the same problem as queueing lengths. The value of workzone user costs is not meaningful to travelers as travel time and queueing delay.

Workzone delays and travel time - as opposed to queue lengths and workzone user costs - provide direct information that travelers can use for trip decisions. Researchers have adopted different approaches to estimate workzone delays and travel time.

Davies et al. (1981) proposed a model to estimate the delays in four different traffic conditions - "free-flow”, "queue starts to form”, “diversion” and "diversionary route at capacity." In the "free-flow" condition, the link demand on the main route is less than its 
capacity and there are only speed reductions through the workzone. In the "queue starts to form" condition, the link demand on the main route is greater than the capacity and no traffic has been diverted. In the "diversion" condition, the traffic starts to divert to the alternate route. In the "diversionary route at capacity", both the main route and the alternate route have reached their capacities. Davies, Vincent and Jacoby’s study was to estimate the impacts due to short-term construction, such as maintenance work.

Cassidy and Han (1993) proposed a model for estimating delays and queue lengths at two-lane highway workzones. When one of the two lanes closed, traffic in both directions needs to share the remaining open lane. This type of lane closure was called “one-way traffic control” by the authors. Because the "one-way traffic control” is a special case, the applications of Cassidy and Han's study cannot be extended to other types of lane closures.

Jiang (1999) reviewed the time-related costs defined in Memmott and Dudek’s study and categorized total delays into four parts: (1) delay due to vehicle deceleration before entering workzone, (2) delay due to reduced speed through workzone, (3) delay for resuming freeway speed after exiting workzone, and (4) delay due to vehicle queues. The total delays were further used to calculate workzone costs. The difference between Jiang's study and Memmott and Dudek’s study is the technique used to estimate delay. Memmott and Dudek used queueing diagrams to calculate queueing delays, while Jiang applied queueing theory. Both of these two studies focus on estimating the total workzone user costs within a specified time period. The total delays calculated in these two studies are with respect to all vehicles in the system during a certain period, instead of for only those vehicles entering the system during the time period. 
The HTIS applies queueing diagrams to model different combinations of arrival rates and derives a general formula for these models. These models can be used to estimate the total delays with respect to vehicles entering the system during a certain time period and average workzone delay per vehicle. The queueing models used in the HTIS are explained in Section 5.5 . 


\section{DATA AVAILABLE}

Several data are required to implement this highway travel information system. The required data include a network file, historical link volumes, initial O-D matrices, and workzone capacity constraints. This chapter explains the uses of these data, the formats of the data obtained and the problems encountered if the data obtained cannot be used directly.

\section{$\underline{3.1 \text { Network File }}$}

\section{A. The uses of network files}

Network files are an important data resource for highway travel information systems. They are large databases that contain information related to highway links, intersections and interchanges such as geographical locations, road classifications, historical link volumes, link lengths, speed limits, and link capacities.

Geographical locations of links and nodes are used to construct highway networks. The geographical location of a highway link is defined by the coordinates of its two end nodes. Road classifications of links are used for forecasting traffic patterns on links during the link volume forecasting process. The traffic patterns for sample links of a road class can represent those links of the same road class under the assumption that the traffic patterns of the same road class are similar. Historical link volumes in the network files 
are used as the basis for link volume forecasting. Link volumes in the near future are forecasted based on historical link volumes. Link lengths and speed limits are used in calculating travel time, which is for use in traffic assignment and O-D estimation. Link capacities are for use in traffic assignment, O-D estimation and delay estimation.

B. The formats of the obtained data

There are two Indiana State network files available: one in TransCAD format and the other in TRANPLAN format. The network file in TransCAD format was obtained from Indiana Department of Transportation (INDOT). The TransCAD file includes all the required data fields of all major highways in the Indiana state network and major arterials in urban areas. Although the data for some of the highway links (such as link volumes) are absent, only a few of the missing data are critical for our analysis.

The network file in TRANPLAN format is from the previous research project (Yang and Fricker, 1996). Although the TRANPLAN file includes only some of the required data for major highways in the Indiana State, it is capable of O-D estimation, using a method called Fast Matrix Calibration. Detailed comparisons of TransCAD and TRANPLAN in terms of record storage capacities and analysis capabilities appear in Chapter 4.

To make use of the advantages of both TransCAD and TRANPLAN, the HTIS attempted to convert the TransCAD network file to TRANPLAN formats, so as to use the capability of Fast Matrix Calibration in TRANPLAN.

The original TransCAD network file contains 761 Traffic Analysis Zones (TAZs), which include 667 township-level internal zones and 94 external zones. In considering the degree of precision, the township-level TAZs may be too detailed for statewide trips. 
The number of trips per day between most pairs of townships in the state is very small. Because processing time increases as the number of TAZs increases, the HTIS planned to combine the township-level TAZs into 134 TAZs, to include 92 county-level internal zones and 42 external zones.

Several tasks were undertaken in the attempt:

(1) Internal TAZ centroid modifications: In combining the township-level internal TAZs to county-level internal TAZs, the township centroids and their centroid connectors need to be removed and new county centroids and centroid connectors need to be added.

(2) External zones adjustments: The number of external zones needs to be reduced as the number of internal TAZs decreases.

(3) O-D matrix modifications: The O-D matrix also needs to be modified correspondingly to match the new identification numbers of the internal and external TAZ centroids.

(4) Modified network verifications: The assigned link flows from the assignment of the modified O-D matrix to the modified network are compared with the ones from the assignment of the original O-D matrix to the original network. The differences between the two assigned link flows should be within acceptable ranges.

(5) Network file conversion: The modified TransCAD network file is converted into the formats of TRANPLAN.

C. The problems encountered

There were several problems encountered as the township-level TAZs were being combined into county-sized TAZs. 
(1) It requires considerable effort and time: Each task mentioned above requires considerable effort and time. In addition, the first four tasks above may need to be repeated if the results of verifications are not satisfactory.

(2) The verification results are not satisfactory: After investing a lot of effort and time, the modified network file and O-D matrix were verified by performing traffic assignment. The results showed a lot of zero-flow links, even on several major roads. Possible causes of this problem include a complicated network system with few centroids and inappropriate centroid connectors.

(3) There are limitations on data storage in TRANPLAN: The TransCAD database contains large amounts of data that TRANPLAN could not handle. The version of TRANPLAN we used limits the total node number to 10,000 , which is less than the 15,223 links in the TransCAD network file.

In conclusion, the attempts to make use of the advantages of both TransCAD and TRANPLAN network files were not successful. The large effort and time required, the unsatisfactory verification results, and limitations on data storage in TRANPLAN make the conversion of TransCAD network file to TRANPLAN format not worthwhile. An alternative to building a complete network file is to use the existing network files in TransCAD or TRANPLAN formats without conversions. Some modifications need to be made when choosing to use either the TransCAD network file or the TRANPLAN network file:

- TransCAD network file: Fill in missing data critical for analysis. No simplifications are made. 
- $\quad$ TRANPLAN network file: Add in required data fields and fill in data for all highway links.

Detailed comparisons of TransCAD and TRANPLAN and the final selection of the software to use appear in Chapter 4.

\section{$\underline{3.2 \text { Historical Link Volumes }}$}

Historical link volumes are the data on the highway link volumes in the past. They are used as the bases for link flow forecasting for specific time frames in the near future. The forecasted values of link volumes for a specific time are treated as the demands on the highway links at that time. These values are then used for delay estimations and O-D matrix estimations.

The available historical link volumes are in the following two formats: Automated Traffic Recorders (ATRs) and county flow maps.

\subsubsection{Automated Traffic Recorders (ATRs)}

ATRs are permanent stations that collect highway link volumes. There are two sources of ATR data: Telemetry stations and Weigh In Motion (WIM) stations. There are 58 Telemetry stations and 35 WIM stations within Indiana. Figure 3.1a shows the old system used before 1999, and Figure 3.1b the new system used after 1999. Each station

records continuous hourly volumes when the detectors and equipment function well. Some missing data due to malfunctions of the detectors and equipment are found in files provided by INDOT (Figure 3.2). 


\subsubsection{County Flow Maps}

County flow maps contain Annual Daily Traffic (ADT) volumes for each road segment in the state highway network. The source of these data is 48-hour traffic counts conducted by INDOT in each county every three to five years. The data in the county flow map are not from the same time frame, because the counts are conducted over a 3to-5-year cycle. The problem with ADTs in different time frames has been solved by INDOT by updating the ADTs in the county flow maps into the same time frame, such as 1995. The updated ADTs for 1995 are available in the "ADTVOL" field of the network file (Figure 3.12).

In order to forecast the hourly traffic patterns of the links, the converted ADTs in county flow maps need to be further converted into hourly format. The HTIS uses the data of ATR stations as the basis for updating and forecasting link volumes in the county flow map. The process is discussed in Section 5.2.

\section{$\underline{3.3 \text { Initial O-D Matrices }}$}

A. The use of initial O-D matrices

Initial O-D matrices are tables recording the number of trips that occurred between each pair of TAZs. The use of initial O-D matrices is one of the inputs of O-D estimation, which generates estimated O-D matrices for specific time periods, based on link volumes for those time periods. The estimated O-D matrices and link capacities with workzone capacity constraints for specific time periods are then used in traffic assignment to determine the link volumes under the scenario in which the road construction locations are known by all road users. 
B. The formats of initial O-D matrices

The initial O-D matrices obtained from INDOT are in two different formats: the daily O-D table and multi-hour O-D tables that are in 3-hour and 18-hour periods. The daily O-D table contains the number of trips between each pair of all TAZs in a weekday. The source of the daily O-D tables is the result of using TransCAD's O-D estimation feature with a seed O-D table, which was developed with available Census, household travel survey and socioeconomic data representative of travel condition in Indiana, and daily link volumes contained in the network file as inputs (Cambridge Systematics Inc., 1998).

The multi-hour O-D tables provided through INDOT, on the other hand, contain only the number of trips between each pair of internal TAZs for periods less than 24 hours, including AM-peak, PM-peak, and off-peak periods. The AM-peak and the PM-peak period each lasts three hours. The off-peak period, which includes the remaining periods in a weekday, lasts eighteen hours. The sources of the multi-hour O-D tables are the results of the daily O-D table applied by the time-of-day factors obtained from the household travel survey data (Cambridge Systematric Inc., 1998).

The process of generating initial O-D matrices is shown in Figure 3.13.

C. The problems encountered

As explained earlier, initial O-D matrices are one of the inputs for O-D estimation. In order to perform O-D estimation, the time period formats of the initial O-D matrices should match those of the link volumes, which are in hourly formats. Therefore, the initial O-D matrices in both daily and multi-hour formats should be converted into hourly formats. 
In addition, the multi-hour O-D tables include only internal trips. The internalexternal and external-external trips are also required for O-D estimation. Therefore, these tables are not suitable in the HTIS.

The daily O-D table, on the other hand, contains internal-internal, internal-external and external-external trips. It can be used for O-D estimation after being converted to hourly trip table. The daily to hourly conversion factor is obtained from the ATR data. The process is discussed in Section 5.3.

\section{$\underline{3.4 \text { Link Capacities }}$}

A. The uses of link capacities

The link capacities under prevailing roadway, traffic, and control conditions are referred as normal capacities (HCM, 1997). The values of link capacities that change due to the effects of road construction are referred as work zone capacities.

The uses of link capacities are for traffic assignment and delay estimation. Link capacities are one of the inputs to traffic assignment. As explained in the use of initial OD matrices, the traffic assignment procedure applying workzone capacities to the links under construction, and normal capacities to all other links, can determine the link volumes under the scenario, in which the road construction locations are known to all road users.

Workzone capacities are also one of the inputs for delay estimation due to road construction. Delay estimation is discussed in Section 5.5. 
B. The formats of road capacities

The normal capacities in the network file provided by INDOT are in two formats: daily and hourly. The hourly normal capacities are in five different levels of service, from level of service A to E. (See Figure 3.14.) The source of these normal capacities is from a research project (Whitford and Opsuth, 1997) that calculated link capacities based on the maximum flow that can be served under a specific level of service defined in the Highway Capacity Manual (HCM, 1997).

Typical workzone hourly capacities have been established by Dudek and Richards’ study (1981). The detailed derivation of workzone capacities is discussed in Section 5.5.

C. The problems encountered

The hourly normal capacities at level of service $\mathrm{E}$ for all lanes in the network file provided by INDOT are used in the HTIS, because the volumes at level of service E are usually treated as capacities. However, the hourly normal capacities in the INDOT file at level of service E are two times higher than the values suggested in HCM (1997). A temporary factor of 0.5 is applied to those values in the network file. 


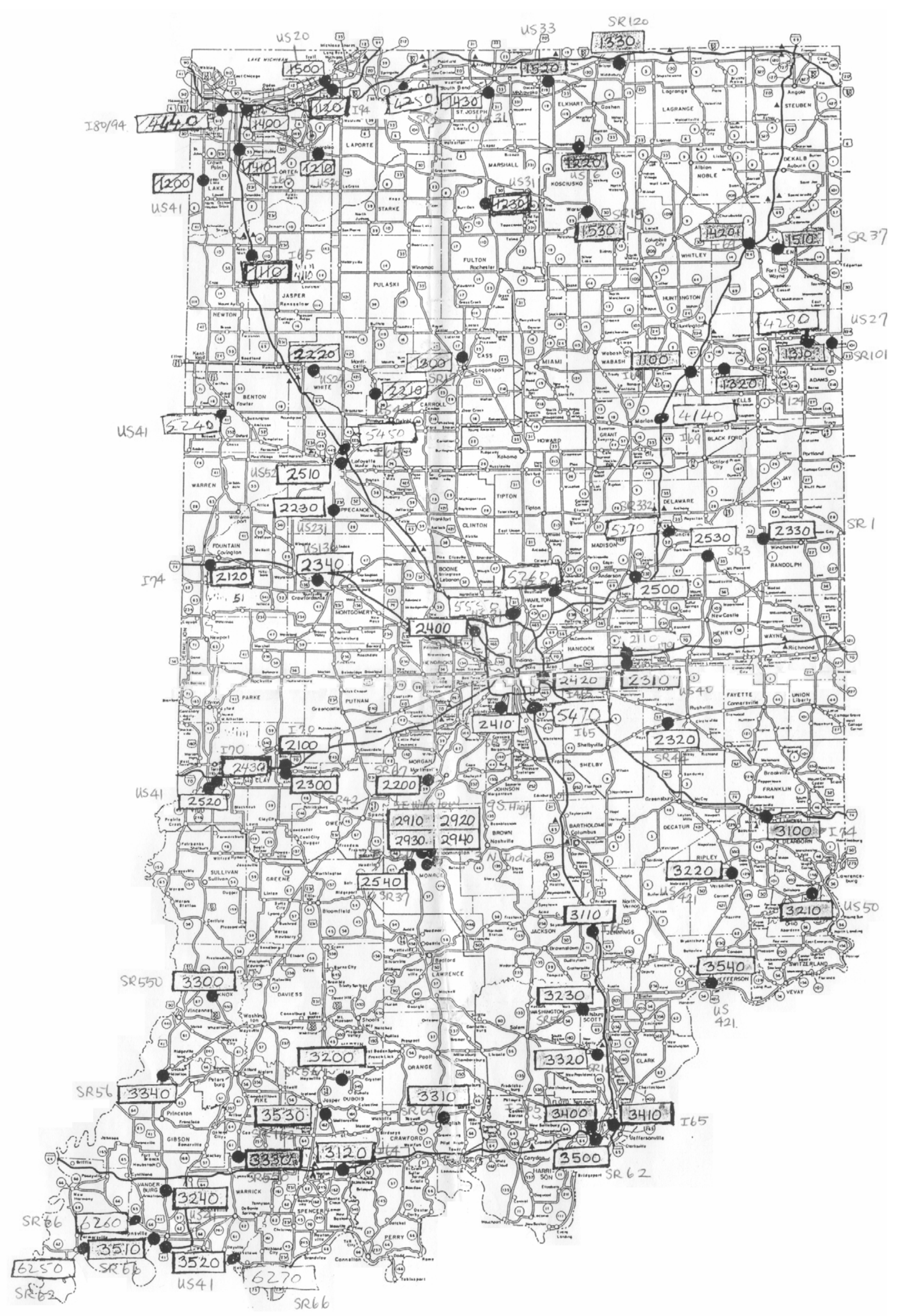

Figure 3.1(a) Locations of ATR stations in Indiana (old system) 


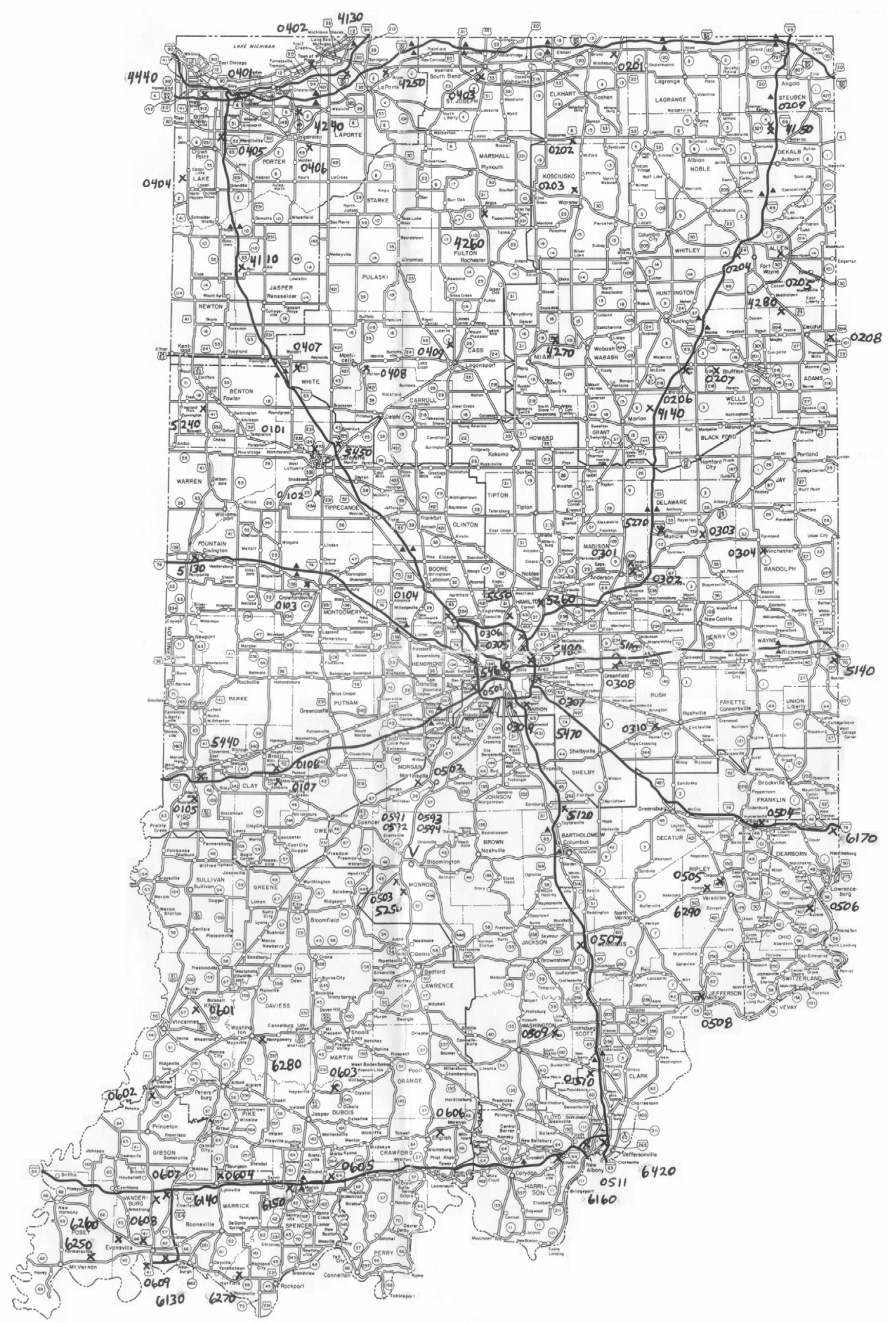

Figure 3.1(b) Locations of ATR stations in Indiana (new system) 


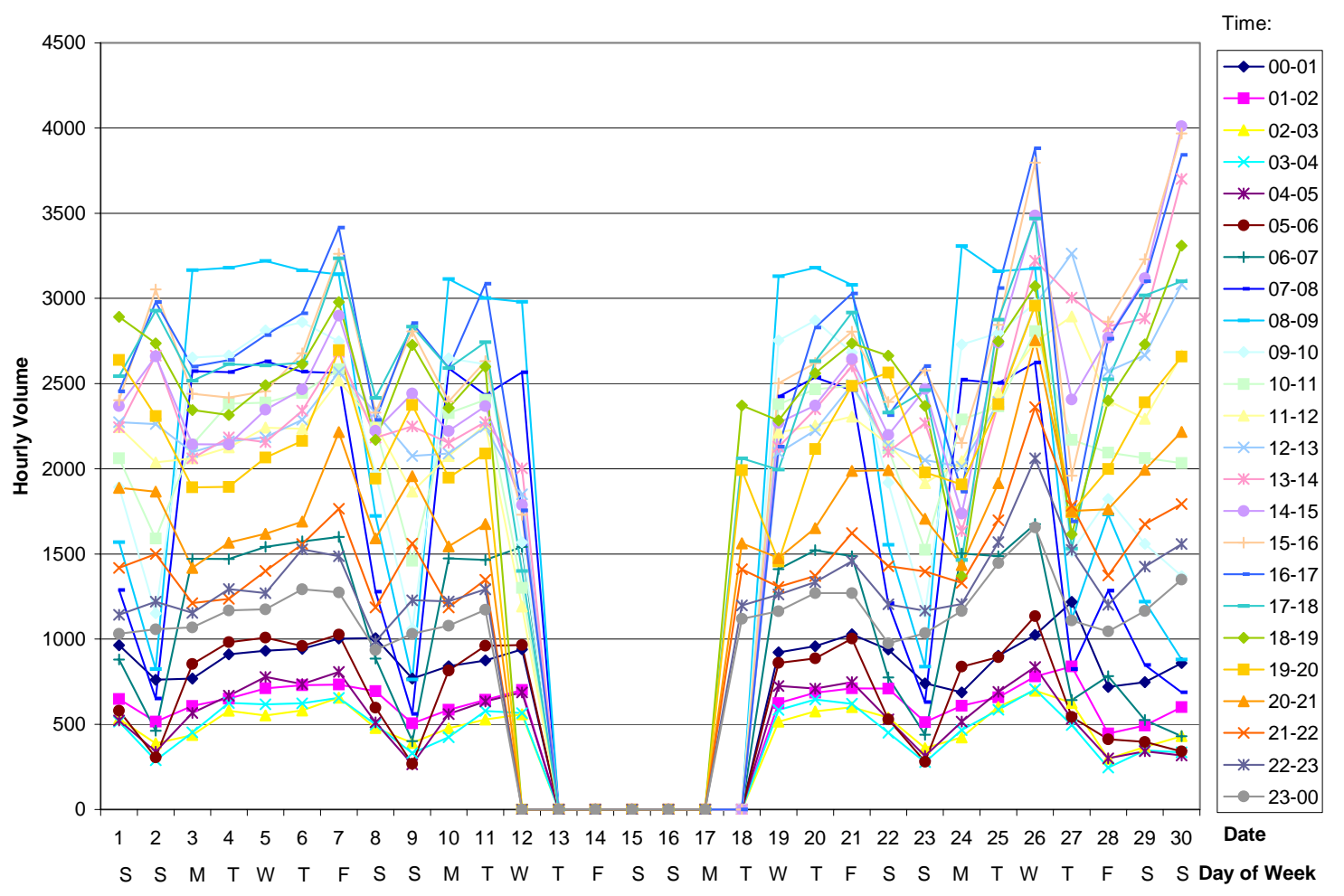

Figure 3.2 Malfunction occurred between November 13 and November 17 in Westbound of ATR Station 1400 in 1997 


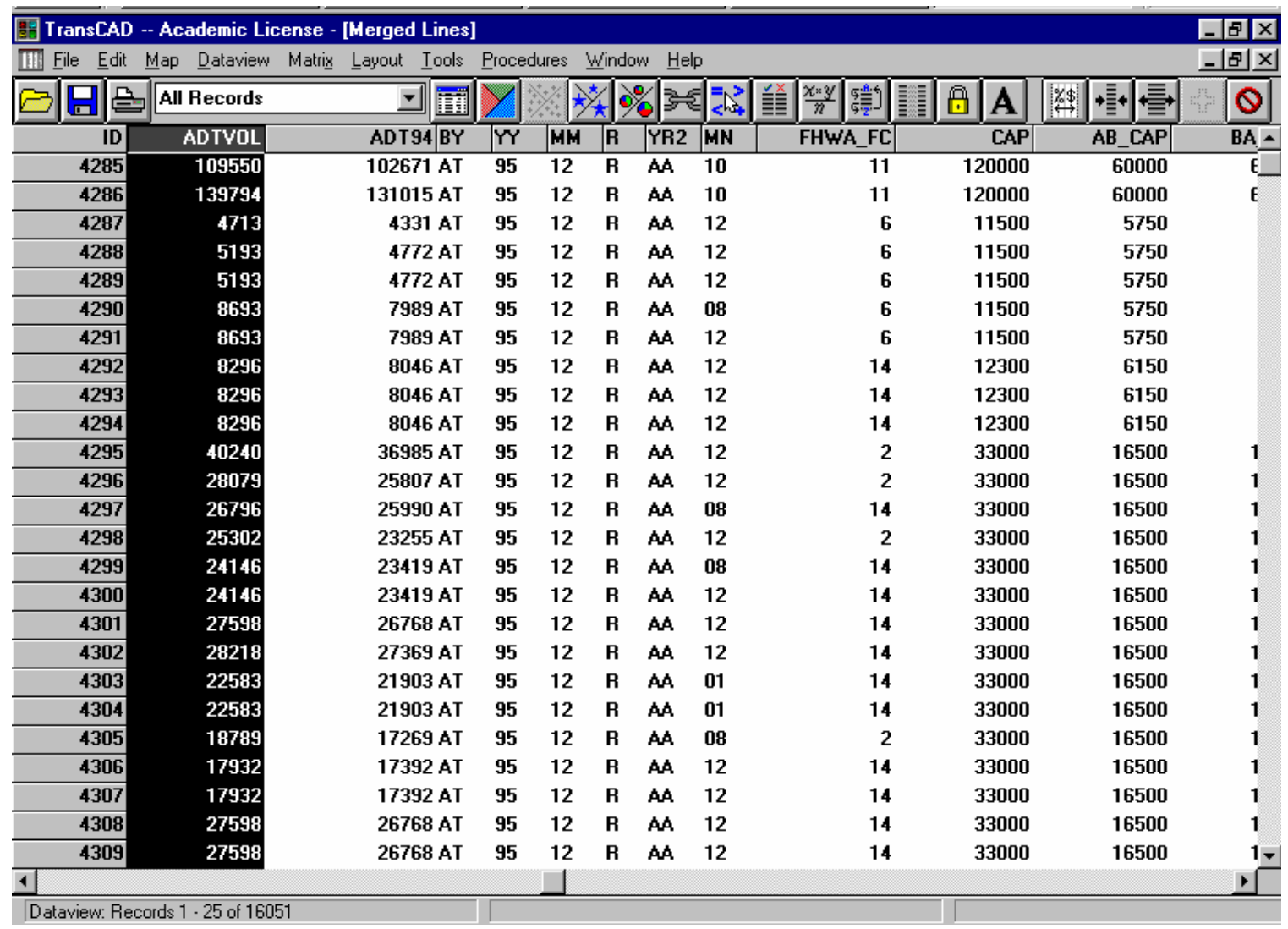

Figure 3.3 Excerpted ADTs in the “ADTVOL” field of a TransCAD network file 


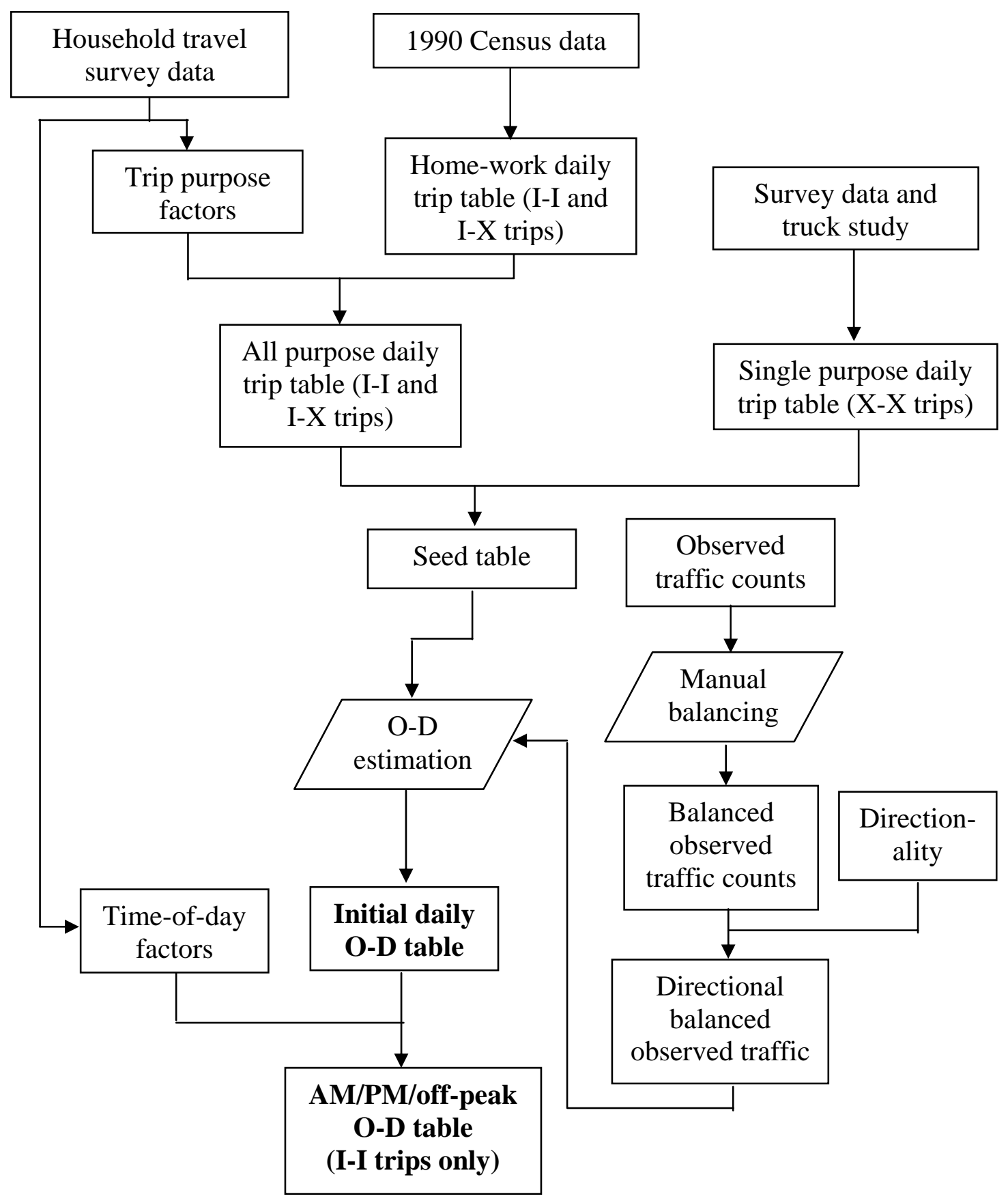

Figure 3.4 The process for generating initial O-D matrices 


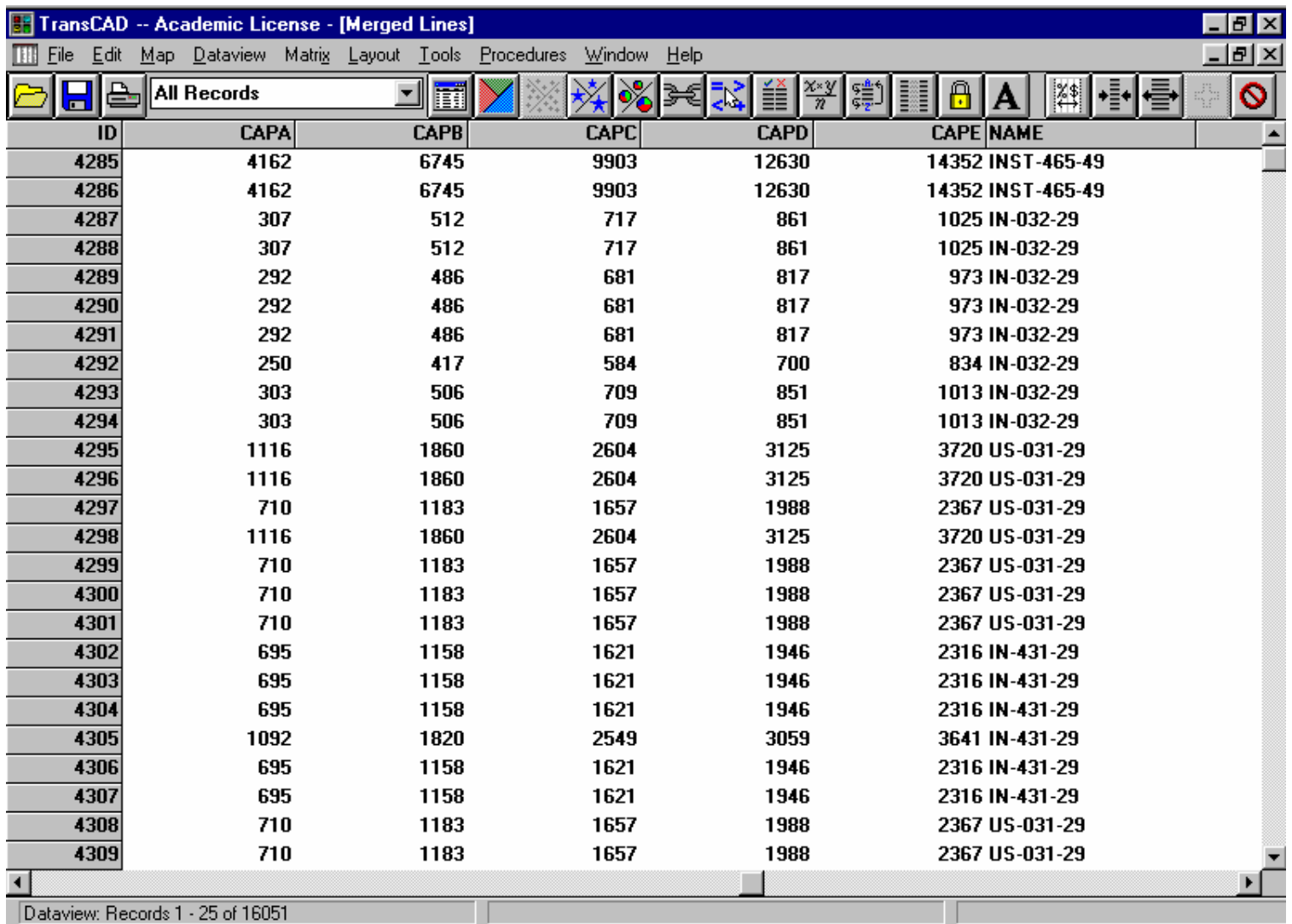

Figure 3.5 Example link capacities for levels of service A to E (CapA CapE) in TransCAD network file 


\section{SOFTWARE APPRAISAL}

Because the HTIS intends to use travel demand modeling procedures to forecast the traffic conditions that could occur on specific links at specified times during the next year, appropriate software tools are necessary to implement travel demand models. The main object of this chapter is to compare two eligible software packages, TransCAD and TRANPLAN, and to suggest an appropriate one for use in this research.

Two main features are considered in making the final decision: the problem-solving functions provided by the package and the software efficiency in implementing the HTIS. The first feature is evaluated by examining the package capabilities in three respects: network capacity, traffic assignment, and O-D estimation. Then, two aspects regarding software efficiency are taken into account: the user interface and the efforts required to build inputs. These considered aspects are elaborated on separately in Sections 4.1 to 4.5. In addition, Section 4.6 gives the conclusion for the discussion.

\section{$\underline{4.1 \text { Network Capacity }}$}

A TRANPLAN study area is limited to 1,500 zones, 10,000 nodes, and node names (expressed as numbers) no greater than 16,000. TransCAD, though not specifying its limitations in the manual, is capable of dealing with networks beyond TRANPLAN's 
limitation on total node numbers and node names. The current TransCAD network file for Indiana contains 761 zones and 15,223 nodes, with a maximum node name 311,326.

\subsection{Capability on Traffic Assignment}

Several methodologies of traffic assignments are developed based on different optimal objectives, such as all-or-nothing, iterative capacity restraint, stochastic, user equilibrium, stochastic user equilibrium, and system optimum, each of which is applied for different purposes (Ortuzar and Willumsen, 1996). Among these traffic assignment methods, user equilibrium and stochastic traffic assignments seemed to be most appropriate for use in the HTIS.

User equilibrium traffic assignment is applied to simulate the "complete information” scenario, in which all drivers have adequate information about road construction zones. Under user equilibrium, no user may reduce his/her travel time by changing the route taken. Therefore, user equilibrium can simulate the situation in which all drivers make their optimal choices based on “complete information.”

Stochastic traffic assignment is well-suited to simulating users’ decisions when facing two or more adjacent parallel routes. Even if one of the routes is superior to the other route(s) in terms of saving time, some users may still choose another one. Unlike all-ornothing traffic assignment, none of the reasonable route alternates will have zero flow when stochastic traffic assignment is used.

Both TRANPLAN and TransCAD provide the user equilibrium and stochastic options for traffic assignment. Therefore, given the same network and the same 
demands, both models can be tested to see if they produce network loadings that are reasonable.

\subsection{Capability for O-D Estimation}

The O-D estimation techniques are needed in the HTIS to build statewide O-D matrices based on observed traffic counts because most existing O-D tables from surveys are prepared for local cities. Mathematically, the solutions (the O-D matrices) are usually not unique, because the number of constraints (the number of traffic counts) is far fewer than the number of unknown variables (the number of O-D pairs) (Ortuzar and Willumsen, 1996).

The objective here is to find an O-D matrix that can produce a flow pattern that adequately replicates the actual traffic situation. The more reliable the traffic counts are, the more likely the resulting $\mathrm{O}-\mathrm{D}$ table is to generate link volumes that are closer to the “reliable” counts. The ability to constrain the O-D estimation procedure to match more closely the more reliable data is a desirable trait.

TRANPLAN uses an add-on function called Fast Matrix Calibration (FMC) that can adjust an existing origin-destination matrix to match given traffic flow constraints at screenlines and zonal boundaries (BGC, 1993). FMC allows users to assign an elasticity value to each screenline and zonal boundary. The elasticity governs the extent to which a screenline and a zonal boundary can be adjusted relative to other screenlines and zone boundaries. A higher elasticity of a screenline or a zonal boundary indicates that the number of observed trips passing through the screenline or the boundary is trustworthier. Therefore, the traffic assignment results based on the estimated O-D matrix should 
produce the number of trips, which pass through the screenline close to the number of trips observed. In this way, O-D matrices are adjusted according to the assigned elastic boundary conditions. FMC can thus provide the user with more control over the results of the O-D estimation.

TransCAD uses a Single Path Matrix Estimation Method (SPME) to estimate O-D matrices from counted traffic by minimizing the average deviation between calculated and counted traffic along the optimal path used by each zone pair. The optional path is calculated by using All-or-Nothing assignment.

SPME solves the problem of unbalanced counted traffic that does not meet node equilibrium by considering traffic counts as stochastic variables. However, because the objective function in SPME is not to minimize the average deviation of the cells in the estimated and old O-D matrices, the patterns of the estimated O-D matrices may be completely different from the patterns of the old O-D matrices, which are usually obtained from household surveys. The results of TransCAD's O-D estimation are hard for the user to control.

\subsection{User Interfaces}

User interfaces are an essential factor in the ease of use of highway travel information systems. The users are those who responsible for building or operating this highway information system, including system maintainers, INDOT personnel, and people authorized to update it. The interfaces refer to the shells that accept human commands to control the functions provided by the software. A friendly user interface can not only 
effectively flatten the learning curve, but also shorten the time to build a new system and make the maintenance easier.

TRANPLAN is a modularized transportation planning software package. Most tasks in TRANPLAN require users to create control files and input files in text format. The control files are executed through a console program under the DOS environment. Like a computer language compiler, the console program always examines the inputs of control files (and generate warning or error messages if there were any) before proceeds the commands. For other tasks demanding a graphic interface to operate, TRANPLAN provides an independent program called the Highway Network Information System (HNIS), which generates and demonstrates geographic plots of network files. Separate modules are also provided to control the database interface and to convert network data from and to ARC/INFO GIS format.

The characteristic of modularizing separate functions in TRANPLAN makes it run efficiently without including unnecessary functions in computer memory. However, users are required to learn several different interfaces and to maintain files for each interface. The learning curve in getting acquainted with several different interfaces is relatively steep and file management is difficult. In addition, TRANPLAN does not provide macro functions to create an independent interface for the travel information system. All operations must be done through executing control files under the DOS environment. The required of level of understanding of TRANPLAN itself is somewhat high, which makes it not very user-friendly.

TransCAD is transportation-oriented Geographic Information System (GIS) software that links the transportation database system to the graphical network. Users not only can 
have a clear view of the graphical presentation of the network data, but also be able to maintain and query network databases, and perform planning analysis procedures under a single Graphic User Interface (GUI). Although TransCAD requires more computer resources to run, because all functions under a single interface are included, it provides macro functions to generate an independent interface for the travel information system. Through the independent interface for the travel information system, users may perform all required operations with only a basic understanding of how to operate TransCAD. Therefore, the user interface of TransCAD is better than that of TRANPLAN for use in the HTIS.

\subsection{Effort to Build Inputs}

The main effort of this research is to implement a highway travel information system. The effort needed to build inputs to a network file for specific software should be reasonable, rather than time-consuming. The best choice of a network file would be the one that is ready for use. However, if the effort to build inputs for a network for specific software is reasonable, the selection of software should be based on all factors, not just the effort to build inputs.

As explained in Chapter 3, the TRANPLAN network file created in a previous research project (Yang and Fricker, 1996) includes only some of the required data for major highways in Indiana, such as link length, speeds, and capacities. Other data, such as historical link traffic counts, road names, road classifications, county codes, and numbers of lanes, are essential to travel information systems and would need to be added to the TRANPLAN network file. The effort to add such inputs to the TRANPLAN 
network file would be considerable. On the other hand, the TransCAD network file obtained from INDOT includes all the required data fields, except for a few missing data on several links. The only missing data that are essential to O-D estimation are historical link traffic counts for some major highways. Only a little amount of time and effort are needed to input those data into the TransCAD network file.

\subsection{Chapter Conclusions}

TRANPLAN and TransCAD were first chosen as the candidates for traffic demand models for the HTIS because they both had statewide network files developed by previous projects. These two candidates were evaluated based on the requirements of this research. The comparisons of these two software packages are summarized in Table 4.1.

Both TRANPLAN and TransCAD provide sufficient options in traffic assignments for use in the HTIS. Although TRANPLAN has the FMC function to provide better control on the O-D estimation results, the lack of sufficient network capacity, a steep learning curve, and the large effort required to build inputs for its network file make it harder to be applied. TransCAD is superior because of its high network capacity, selfbuilt and friendly user interface, and ready-to-use network file. Although TransCAD does not allow user control of the outcome of O-D estimation, the capabilities of TransCAD are otherwise excellent for use in the HTIS.

Moreover, as discussed in Chapter 3, the available Indiana State network files are in TransCAD format and in TRANPLAN format. To make use of the advantages of both software packages, the HTIS first attempted to convert INDOT's TransCAD network file to TRANPLAN format. However, the conversion process could not be successfully 
accomplished because of the large amount of effort and time required, the disappointing verification results, and limitations on data storage in TRANPLAN. Therefore, the options are to use either the TransCAD network file or the TRANPLAN network file, but not to attempt a conversion. The first alternative is preferable because the network file in TRANPLAN format is too simplified and it lacks of several essential information on

links.

Based on the comparative evaluation described in this chapter, TransCAD was selected as the traffic demand model to be used in the HTIS. 
Table 4.1 Comparisons of TRANPLAN and TransCAD as the selected traffic demand software for the highway travel information system

\begin{tabular}{|l|c|c|}
\hline \multicolumn{1}{|c|}{ Capability } & TRANPLAN & TransCAD \\
\hline Network capacity & & $\checkmark$ \\
\hline Traffic Assignment & $\checkmark$ & $\checkmark$ \\
\hline O-D estimation & $\checkmark$ & 0 \\
\hline User Interface & & $\checkmark$ \\
\hline Efforts to build inputs & & $\checkmark$ \\
\hline
\end{tabular}

$$
\begin{gathered}
\text { (note) } \checkmark: \text { excellent } \\
\text { O: good }
\end{gathered}
$$




\section{METHODOLOGY}

The methodology used in the HTIS is described in this chapter. This research applies traffic volume forecasting techniques, traffic demand models and delay estimation to forecast delays due to scheduled road construction. Traffic volume forecasting techniques are applied to obtain forecasted link volumes before construction. O-D estimation and traffic assignment, which are elements of traffic demand modeling, are applied to forecast link flows after workzone traffic reaches a steady state. Delay estimation forecasts the delays during the transition period before workzone traffic reaches a steady state.

\section{$\underline{5.1 \text { Framework }}$}

A key element of the HTIS is to predict traffic conditions during road construction. As discussed in Chapter 2, several previous studies attempted to predict traffic conditions during road construction. However, some traffic models are too complicated to be implemented on the scale of an entire state, and some do not account for the dynamic changes in the traffic conditions during construction. In order to implement the travel information system for an entire state and have travel information available to the public based on a desired departure time entered by a traveler, a more practical approach is required. 
The HTIS introduces an approach called Workzone Delay Equilibrium Estimation (WDEE) that uses relatively little data to predict the dynamic traffic conditions that occur during construction. As explained in Chapter 1, WDEE involves three steps. First, two extreme values of link volumes are calculated based on two scenarios: "no information", in which no drivers know about a new reduction road in capacity, and "complete information", in which all drivers have adequate information about road construction zones. Next, the delays for the "no information" and "complete information" scenarios are estimated. Finally, the expected delays on each link as a function of time during construction are estimated based on the estimated link volumes. The framework for the HTIS is shown in Figure 5.1.

A. Link volumes for "no information" scenario

The "no information" scenario depicts road conditions when no drivers have information about road construction zones. This scenario is to simulate the condition at the beginning of a road construction if no drivers know that the new construction zone exists. Drivers will follow the same routes they normally use during the same time frame, unaware of any constrained capacities of some links on their routes. Therefore, the traffic demands on each link for a specific time frame with no construction and at the beginning of road construction are treated the same under "no information" scenario. For example, there were $2,000 \mathrm{vph}$ on a highway section when there was no construction. Because of construction, the capacity of the highway decreased from 3,600 vph to 1,700 vph. The 2,000 drivers did not have the information about the construction, so they kept on traveling on the same route, even though the capacity had been drastically reduced. 
The travel demand on each link for a specific time frame in the near future can be estimated by applying a traffic volume forecasting technique proposed by the HTIS. This technique first converts the ADTs in the TransCAD network file to an hourly basis as base-year traffic demands. Then the data from ATR stations are used to find peak period factors and growth factors for different road classes in different months. The link demands of a direction for a specific time frame in the near future are obtained by applying the peak period factors, growth factors and directionality to the base-year traffic demands. The traffic volume forecasting technique is discussed in Section 5.2, "Traffic Volume Forecasting."

B. Link volumes for "complete information" scenario

The "complete information" scenario assumes all road users have adequate information about road construction locations. This scenario simulates the case in which road construction has been proceeding long enough that all drivers considering use of that road know about it. Drivers make informed decisions about whether to stay on the original route or divert to alternate routes, based on their knowledge of the workzone and the surrounding network. Although some drivers may choose to departure at different time to minimize delays, this situation is not reflected in the "complete information" scenario. Under the assumption that drivers will only divert to alternate routes rather than departure at different time to minimize delays, the flows on each link under the "complete information" scenario may be different from the flows under the "no information" scenario. The travel demand between each O-D pair for a specific time period in a specific day type, however, is assumed to be the same for both the "complete information" and "no information" scenarios. 
The demand for trips between each O-D pair during a specific time frame is represented by an O-D table. By using the link volumes for the "no information" scenario and initial O-D matrices as inputs, the demand for trips between each O-D pair for a specific time frame can be estimated. This process is discussed in Section 5.3, "OD Estimation."

The link flows for the "complete information" scenario are obtained by assigning the demand for trips between each O-D pair for a specific time frame, the estimated O-D table from O-D estimation, to a network having work zone capacity constraints. The traffic assignment process is intended to simulate the conditions in which all drivers make informed decisions to minimize delays such that no drivers can save any time by changing their routes. This process is discussed in Section 5.4, "Traffic Assignment." C. Delays for "no information" and "complete information" scenarios

The delays on each link for both "no information" scenario and "complete information" scenario during the specific time frame are calculated based on queueing theory. The condition in which demand exceeds capacity is assumed to cause queueing delay. The delays for the "no information" scenario are treated as the upper bounds of possible expected delays, and the delays for the "complete information" scenario are treated as the lower bounds of possible expected delays. The two values form the possible range of delays, with the expected delays falling in between. The delay estimation model is discussed in Section 5.5.1.

D. Expected delays on each link in relation to age of workzone

The amount of delay on a link is related to the volume of the link. In queueing theory, it is assumed that delay occurs on a link when its volume is greater than its 
capacity. When delay occurs, the delay on a link decreases as the link volume decreases. By building the relationship between the volumes of a link at a workzone, the expected delay on the link can be estimated.

The link volume at a workzone is expected to decrease as construction proceeds and more drivers become aware of the construction. Some of the informed drivers may choose to divert to alternate routes. On those roadways with significant through traffic by infrequent drivers, there may always be a certain percentage of "uninformed" drivers. It is these drivers who could benefit most from a pre-trip HTIS.

The effects of diversion on volumes can be related to the time since construction began. At the beginning of construction, only a few drivers know about the situation and only some of the informed drivers decide to divert to alternate routes. At this time, most of the drivers remain on the main routes. The link volumes are close to the upper bounds, which are obtained from the "no information" scenario. The expected delays estimated based on the "no information" link volumes are close to the upper bounds. As the construction proceeds, more drivers become aware of the construction and some of them divert to alternate routes. When the construction has proceeded for a certain period of time, most of the drivers are aware of the construction and the diversion process reaches a "steady" state. The link volumes are close to the lower bounds, which are obtained from the "complete information" scenario. The expected delays estimated based on the "complete information" link volumes are closer to the lower bounds. The closeness to the lower bounds depends on the percentage of frequent drivers. The higher the percentage of frequent drivers is, the closer the resulting expected delays are to the lower bounds. For the expected delays in between the beginning of a construction and the 
"steady" state, a normalized volume-time relationship found from historical ATR data is applied to the upper and lower bounds of link volumes to dynamically estimate the link volume in relation to the age of the construction zone, which is then used to obtain the expected delay. (See Section 5.5.2 for details.)

\subsection{Traffic Volume Forecasting}

Traffic volume forecasting is used to forecast the flow on each link for the "no information" scenario, based on historical data to simulate the condition at the beginning of construction. As explained in Section 5.1, under the "no information" scenario, no drivers know about the construction, thus all drivers will follow the same routes as they normally do. As a result, the demands on each link at the beginning of construction will equal the demand on each link with no construction. The demands at the beginning of a construction project for different day types and different periods within a day can be forecasted based on the historical data when there was no construction.

The links required to do traffic volume forecasting include all links in Indiana. Although only the links under construction are concerned in the HTIS, all the other links that are not under construction are also required to do volume forecasting. The flow on each link is needed to estimate O-D tables for use in assigning traffic flows to each link under the "complete information" scenario.

The process for forecasting volumes of all links in Indiana using advanced traffic volume forecasting techniques such as time series models involves two steps: building forecast models for all ATR stations and forecasting volumes for all links without ATR stations on them. 
The process of building forecast models for each ATR station involves two steps: data classification and model building. Data classification is to classify the ATR data into several groups, each of which has similar traffic patterns. The traffic patterns can be classified into three categories: normal days, holidays, and special days.

(1) Normal days

Normal days include all days except holidays and special days. There are two types of traffic patterns on normal days: weekday patterns and weekend patterns. Weekday patterns have distinct AM-peak and PM-peak periods, while weekend patterns tend to have a longer and flatter peak period. Figure 5.2 shows the traffic patterns of a typical weekday and weekend at an ATR station located on the northwest side of Indianapolis in November 1997.

\section{(2) Holidays}

The traffic patterns of holidays are more complicated than those of normal days. Not only do the patterns during holidays change, but the patterns of the days and the patterns of the weeks before and after holidays change. The patterns during holidays have lower peaks, while the patterns of the days before and after holidays tend to have higher peaks when compared with the patterns of normal days. Figure 5.3 shows the traffic patterns of the weeks before, during, and after the Thanksgiving Holiday at the ATR station located on the northwest side of Indianapolis in 1997.

(3) Special days

Special days are the days when there are special events happening on a regular basis, such as the annual Indy 500 auto race. Special days are different from holidays in their geographic areas of influences, because the special events often affect local traffic only. 
Figure 5.4 shows the traffic patterns on the days at the ATR station located on the northwest side of Indianapolis from Saturday 3 May to Tuesday 27 May 1997 when the Indianapolis 500 auto race took place. The peak traffic appeared on 25 May through 27 May, because rain caused the final race day to be rescheduled twice. Only a few other ATR stations in the region were noticeably affected by that event.

For each category described above, more than one type of traffic pattern is involved. It is necessary to further classify them into more similar traffic patterns. The data in each category were tested for similarity within the category using the Spearman Rank Correlation Test. For each group of data that is shown to have high similarity, a forecasting process such as Time Series Analysis can be applied to predict link volumes in the near future.

\section{(1) Spearman Rank Correlation Test}

The Spearman Rank Correlation Test (Scheaffer and McClave, 1990) is a distribution-free analog of correlation analysis that does not make any assumptions about the distribution of the underlying data. It can be applied to compare two independent random variables, each at several levels (which may be discrete or continuous). Spearman's method works by assigning a rank to each observation in each group separately. Then, calculate the sums of the squares of the differences in paired ranks $\left(d_{i}^{2}\right)$ according to the formula:

$$
r_{s}=1-\frac{6 \times\left(d_{1}^{2}+d_{2}^{2}+\cdots+d_{n}^{2}\right)}{n \times\left(n^{2}-1\right)}
$$

in which $n$ is the number of observations. 
The Spearman's $r_{s}$ coefficient indicates agreement. A value of $r_{s}$ near one indicates good agreement; a value near zero, poor agreement.

The HTIS applied the Spearman Rank Correlation Test on the data of ATR station 2400 , which is located just northwest of Indianapolis, during November 3 and November 9 in 1997. The results are shown in Table 5.1. The shaded cells indicate high agreement among weekday traffic and that there exist constant trends in the weekday traffic patterns. The weekend traffic patterns, on the other hand, show less agreement with weekday traffic patterns and with themselves.

Overall, those groups of traffic volumes that show high agreement in the Spearman test, statistically, are considered to have similar and predictable patterns, making them ready for use in forecasting traffic trends.

\section{(2) Time Series Analysis}

The HTIS applies the autoregressive integrated moving average (ARIMA) models in time series analysis (Abraham and Ledolter, 1983) to forecast the patterns of the groups of traffic volumes that show high agreement in the Spearman test. The ARIMA models assume a series is correlated if the data are collected sequentially in time. Define $Z_{t}$ as an observed time series $\left(Z_{1}, Z_{2}, \ldots, Z_{n}\right)$. The correlation between a series $\left(Z_{t}\right)$ and the same series with a time lag of $K\left(Z_{t-K}\right)$ is defined as the Autocorrelation Function (ACF), $\rho_{K}$.

$$
\rho_{K}=\frac{\operatorname{COV}\left(Z_{t}, Z_{t-K}\right)}{\sqrt{\operatorname{VAR}\left(Z_{t}\right) \cdot \operatorname{VAR}\left(Z_{t-K}\right)}} K=0,1,2, \ldots
$$

By observing the ACFs of a series, a certain type of ARIMA model can be determined to best describe that series. The patterns of those ACFs that are larger than their $\pm 2 \sigma$ limits should be considered when determining the forecast model. When the 
ACFs are less than the $\pm 2 \sigma$ limits, they are considered "white noises" (uncorrelated random variables) and will not have significant effects on the forecast model.

An example series of hourly volumes was used to implement an ARIMA model. The sample series is the first 160 weekday hourly volumes at ATR station 2400 in November 1997 (Figure 5.5). This series shows a "seasonal" pattern, which repeats every 24 hours. Its ACFs decay exponentially for the first few periods, with large peaks starting from period 24 and small peaks from period 12 repeating every 24 periods (Figure 5.6). The two peaks of ACFs do not decay quickly, which indicates the possibility of requiring one step of seasonal difference at time lag 24 .

Define $B^{m}$ as the backshift operator with a time lag of $m$ such that $B^{m} Z_{t}$ equals $Z_{t-m}$. The ACFs of the series with one step of seasonal difference of order $24,\left(1-B^{24}\right) Z_{t}$, are shown in Figure 5.7. The ACFs decay exponentially for the first few periods and regain a peak at period 24. The possible models based on the ACFs of the series $\left(1-B^{24}\right) Z_{t}$ are $(1,0,0)(1,1,0)_{24},(1,0,0)(0,1,1)_{24},(1,0,0)(2,1,0)_{24},(1,0,0)(1,1,1)_{24}$, and $(1,0,0)(0,1,2)_{24}$. The six numbers in each model represent the parameters for autoregression variables, number steps of difference, the parameters for moving average variables, the parameters for seasonal autoregression variables, number of steps of seasonal difference, and the parameters for seasonal moving average variables. The time lag of the seasonal difference (24) is shown as the subscript for the second parentheses. Among these models, the model $(1,0,0)(1,1,1)_{24}$ has the highest probability for its residuals to be white noise (0.7819) and the smallest residual mean square (1536.81). It is chosen to be the initial forecast model. 
The ACFs of the initial model are shown in Figure 5.8. The ACF at period 25 exceeds its $\pm 2 \sigma$ limits. An additional parameter might be necessary. By adding one more parameter, the model becomes $\left(1,0,1_{25}\right)(1,1,1)_{24}$ (The parameter for moving average variables at period 25 is noted in subscript). The probability of the residuals of this model to be white noise is 0.8848 and the residual mean square for this model is 1451.865. Both values indicate the model $\left(1,0,1_{25}\right)(1,1,1)_{24}$ is better in interpreting the series.

The model $\left(1,0,1_{25}\right)(1,1,1)_{24}$ is further overfitted to check if it requires more parameters to interpret the series. The overfitted model $\left(1,0,1_{25}\right)(1,1,2)_{24}$ shows higher probability of its residuals to be white noise $(0.9555)$ and lower residual mean square (1391.495). It is selected as the final forecast model. The ACFs for the final model is shown in Figure 5.9. All ACFs are now within the $\pm 2 \sigma$ limits.

The weekday hourly volumes for the 48 periods after the first 160 periods at ATR station 2400 in November 1997 were forecasted using the model selected above. To simulate the actual situations in the real world, two sets of forecasting strategies were applied: one without knowing further information (forecasting base 160), the other with the first 24 periods of the following 48 periods known as updated information (forecasting base 184). The former simulates the situation at the beginning of the forecasting process, while the latter simulates the situation when updated information is available and can be used to update the forecasts accordingly without having to rebuild the forecasting model.

The forecast results and the $95 \%$ upper and lower confidence limits of the forecasts are shown in Figure 5.10 and Table 5.2. Both the forecasted traffic patterns for the 
forecasting base 160 and for the forecasting base 184 are very close to the actual values. For the 48-periods-ahead forecasts at the base period 160, the patterns of the weekday hourly traffic volumes are successfully described. The update process helps to pull the forecasts of the first few periods beginning at period 184 closer to the actual values.

The number of forecast models required for a specific month depends on the number of ATR stations and the number of day types in the month. The number of ATR stations within Indiana is 93 . If there are two day types (weekdays and weekends) for a specific month, the total number of forecast models required in the month is 186 .

The second step is to forecast volumes of all links without ATR stations on them. By defining the "area of influence" of each ATR station, the forecast models for each ATR station are applied to those links within the "area of influence" of that ATR station. The "area of influence" of an ATR station is defined by its geographical locations and the road class of the link on which it is located. However, the forecast models of an ATR station cannot be applied to links without ATR stations directly, because the forecast models require historical hourly volumes as forecast bases. Instead, the hourly volumes of those links without ATR stations are obtained by scaling the forecasts of hourly volumes for the ATR station according to the ratio of the ADTs for those links without ATR stations to the ADT for the ATR station under the assumption that the traffic patterns of the former and the latter are similar. For example, assume that the average ADT for ATR station 2400 is 17,000 vehs during weekdays in November, and the ADT for a link near ATR station 2400 is 20,000 vehs during the same periods. The time series model for ATR station 2400 is applied to the historical data for ATR station 2400 to obtain its hourly patterns in November. The hourly patterns for ATR station 2400 in 
November are multiplied by the ratio of $1.18(=20,000 / 17,000)$ to obtain the hourly patterns for the link near the ATR station.

Although the forecasting results using time series analysis successfully depicted the actual traffic trends, the total processing time for ATR station 2400 for a typical weekday during November 1997 was over 6 hours. The time for building all forecast models for a specific month could be over a month. In addition, time series analysis requires historical hourly volumes as forecast bases to apply time series analysis to forecast hourly volumes.

In considering a reasonable time to do traffic volume forecasting, the HTIS adopted an approach that use two available sources of link volume data: from ATR stations and from county flow maps. As explained in Section 3.2, the data from the 92 ATR stations are continuous hourly volumes. The data from county flow maps covering all links within Indiana are Annual Average Daily Traffic (AADT) volumes obtained from different years. The data from county flow maps have been converted to the same base year (1995) in the TransCAD network file. The data from ATR stations are excellent for delay estimation. However, there are only 92 ATR stations within Indiana. The data from county flow maps, on the other hand, cover all links on the Indiana highway system. However, the AADT format needs to be further subdivided in order to estimate delays during different periods within a day. Therefore, the HTIS uses the ATR data to estimate the peak-period traffic patterns and applies them to the data from county flow maps to estimate delay for different periods of time and different day types.

The way to link the ATR data to the county flow map data is referred to in the Traffic Monitoring Guide (TMG) (FHWA, 1995). The TMG groups ATR data based on road classes and calculates aggregate parameters such as mean and coefficient of variance for 
each group of ATR data. Following the same concept of using a subgroup of data from ATR stations belonging to the same road class to represent the characteristics of the road class, the HTIS assumes that the traffic patterns for links of a road class can be represented by the traffic patterns for the subgroup of ATR data belonging to the same road class.

According to the TMG, the minimum groups of ATR data based on road classes should include at least five classes: Interstate Rural, Other Rural, Interstate Urban, Other Urban, and Recreational. The first four groups are defined by FHWA functional codes for highway. The Recreational road class does not specifically define corresponding FHWA functional codes and requires the use of subjective judgment and knowledge of the travel characteristics of the State. Because it is not the interest of the HTIS to distinguish recreational highways from other highways, only the first four road classes are used to group the ATR data.

In addition to road class, the HTIS further classifies the ATR data based on day types. According to the Spearman Rank Correlation Test mentioned in Section 3.2, the data for weekdays at an ATR station exhibit high similarity, and so do the data for weekends. In some cases, such as holidays, the ATR data for those days do not have similarity to other day types. From the test results, the basic day types for ATR data are set to be weekdays and weekends. Additional day types may be added, depending on how may holidays there are in each month and how these holidays affect the patterns.

For each group of ATR data classified based on road classes and day types, the traffic patterns are represented as three periods: 3-hour AM-peak, 3-hour PM-peak, and 18-hour off-peak periods. The 3-hour AM-peak and PM-peak periods reflect the characteristic of 
statewide trips, which take a longer time to complete. The hourly volume within each 3hour period is represented by the average volume of the period.

The procedures for traffic volume forecasting include raw data screening, average peak period factors estimation, directionality estimation, growth factor estimation, and link volumes forecasting. Each procedure is explained as follows:

A. Raw data screening

Before calculating average peak period factors, any bad ATR data due to malfunctions of equipment must be excluded. The ultimate screening for bad data requires expert judgment. However, some bad data can be screened out automatically before manual judgment is applied. The HTIS uses three rules to screen out bad data:

- Some ATR data have exactly the same hourly volumes for both directions for the entire 24 hours in a day. This problem might be caused by data transmission errors. The data for both directions may have been accidentally copied from one of the directions. Normally, ATR data should have different hourly volumes in each direction. To detect and exclude this kind of bad data, a rule is generated: if the sums of volumes for each day for each station for two directions are the same, the data are excluded.

Define $V o l_{D L N J}$ as the hourly volume of direction $D$ at station $N$ for day type $L$ during hour $J . D_{I}$ and $D_{2}$ are the two directions for the traffic at station $N$. The data that match the following criteria are excluded.

$$
\sum_{J} \operatorname{Vol}_{D_{1} L N J}=\sum_{J} \operatorname{Vol}_{D_{2} L N J}, J \in\{0,1, \ldots, 23\}
$$


- Some ATR data recorded consecutive zero flows in one direction for a long period of time. These data needs to be excluded. For example, the records of ATR station 1100 on 19 May 1997 show volumes of 280 and 287 for the hours beginning at midnight and 2:00AM, and 0 for the rest of the day. This problem might be caused by malfunctions of recorders on all lanes in one direction during a day. Although zero flows may appear at some low-volume stations during offpeak periods, the zero flows usually do not last for a long period of time. To screen out this kind of bad data while avoiding the misinterpretation of true zeroflows cases at some low-volume stations, the HTIS adopts a rule: if the sums of the volumes for the entire AM period or for the entire PM period of a day in any direction at a station in a day equal zero, the data should be excluded. Any ATR data that meet the following criteria are excluded. That is, whenever the data for the entire AM or PM period in one direction equal zero, the data for both directions are discarded. The data of the opposite direction alone cannot be used to calculate the directionality of the station even if they are recorded normally.

$$
\sum_{J=0}^{11} V o l_{D_{1} L N J} \cdot \sum_{J=12}^{23} \operatorname{Vol}_{D_{1} L N J} \cdot \sum_{J=0}^{11} \operatorname{Vol}_{D_{2} L N J} \cdot \sum_{J=12}^{23} V o l_{D_{2} L N J}=0
$$

- Some ATR data exhibit unusual high directionality. This might be caused by malfunctions of recorders on a portion of multiple lanes in one direction. Although the data can pass the above screening process, the data should be excluded. To screen this kind of bad data, a rule is formulated to exclude the ATR data whose directionality is greater than $80 \%$. The $80 \%$ directionality is chosen based on the cumulative frequency of the hourly volumes for all ATR 
stations during the AM-peak periods in May, 1997 (Figure 5.11). The cumulative percentage of number of ATR stations for the directionality that is greater than or equal to $80 \%$ is over $95 \%$, which means the percentage of links whose directionality is greater than or equal to $80 \%$ is less than $5 \%$. By assuming that the malfunction rate at ATR stations is $5 \%$, the links with directionality greater than $80 \%$ are excluded. This criterion is formulated as follows:

$$
\begin{aligned}
& \frac{\sum_{J=0}^{23} V o l_{D_{1} L N J}}{\sum_{D} \sum_{J=0}^{23} V o l_{D L N J}}>80 \%, \text { or } \\
& \frac{\sum_{J=0}^{23} V o l_{D_{2} L N J}}{\sum_{D} \sum_{J=0}^{23} V o l_{D L N J}}>80 \% .
\end{aligned}
$$

B. Average peak period factors estimation

The average peak periods for a group of ATR data are defined as the most common peak periods for all ATR stations within that group. In order to find the average peak periods (AM and PM) for a group of ATR data, the peak periods for each station within the group are first found.

For each ATR station within a specific group, the 3-hour AM-peak and PM-peak periods in a day are determined by the maximum three-consecutive-hour volumes in both directions. That is, for each group of road class $M$ and day type $L$, define $I A M_{M L} \equiv(A M$ peak start time) ${ }_{M L}$

$$
\text { where } \sum_{D} \sum_{J=I A M_{M L}}^{I A M_{M L}+2} \sum_{N \in M} V o l_{D L N J}>\sum_{D} \sum_{J=J_{1}}^{J_{1}+2} \sum_{N \in M} V o l_{D L N J}, I A M_{M L}, J_{I} \in\{0,1, \ldots, 9\}, I A M_{M L} \neq J_{I}
$$


The above inequality represents the maximum three-consecutive-hour volumes in both directions between midnight and noon.

Similarly, define $I P M_{M L} \equiv(P M \text { peak start time })_{M L}$

$$
\text { where } \sum_{D} \sum_{J=I P M_{M L}}^{I P M_{M L}+2} \sum_{N \in M} V o l_{D L N J}>\sum_{D} \sum_{J=J_{2}}^{J_{2}+2} \sum_{N \in M} V o l_{D L N J}, I P M_{N L}, J_{2} \in\{12,13, \ldots, 21\}, I_{2} \neq J_{2}
$$

The above inequality represents the maximum three-consecutive-hour volumes in both directions between noon and midnight.

The average peak period factor for road class $M$ and day type $L$ is defined as follows:

$$
\text { (AM peak period factor) })_{M L}=\frac{\frac{1}{3} \sum_{D} \sum_{N \in M} \sum_{J=I A M}^{I A M} V O M_{D L N J}+2}{\sum_{D} \sum_{N \in M} \sum_{J=0}^{23} V o l_{D L N J}}
$$

where $\sum_{D} \sum_{N \in M} \sum_{J=I A M}^{I A M_{M L}} V_{M L N J}^{+2}=l_{D L N}=$ the sum of the maximum three-consecutive-hour volumes of both directions between midnight and noon for all stations $N$ that belong road class $M$, $\sum_{D} \sum_{N \in M} \sum_{J=0}^{23} V o l_{D L N J}=$ the sum of 24-hour volumes of both directions for all stations that belong to road class $M$.

$(P M \text { peak period factor })_{M L}=\frac{\frac{1}{3} \sum_{D} \sum_{N \in M} \sum_{J=I P M_{M L}}^{I P M_{M L}+2} V o l_{D L N J}}{\sum_{D} \sum_{N \in M} \sum_{J=0}^{23} V o l_{D L N J}}$ 
where $\sum_{D} \sum_{N \in M} \sum_{J=I P M_{M L}}^{I P M_{M L}+2} V o l_{D L N J}=$ the sum of the maximum three-consecutive-hour volumes of both directions from noon to the midnight for all stations $N$ that belong to road class $M$.

Off-peak periods include all periods in a day other than AM-peak and PM-peak periods. The off-peak period factor for road class $M$ and day type $L$ is defined as follows:

$$
\text { (Off peak period factor })_{M L}=\frac{\frac{1}{18} \sum_{D} \sum_{N \in M}\left(\sum_{J=0}^{I A M_{M L}-1} V o l_{D L N J}+\sum_{J=I A M_{M L}+3}^{I P M_{M L}-1} V o l_{D L N J}+\sum_{J=I P M_{M L}+3}^{23} V o l_{D L N J}\right)}{\sum_{D} \sum_{N \in M} \sum_{J=0}^{23} V o l_{D L N J}}
$$$$
\text { where } \sum_{D} \sum_{N \in M}\left(\sum_{J=0}^{I A M_{M L}-1} V o l_{D L N J}+\sum_{J=I A M_{M L}+3}^{I P M_{M L}-1} V o l_{D L N J}+\sum_{J=I P M_{M L}+3}^{23} V o l_{D L N J}\right)=\text { the sum of hourly }
$$

volumes of both directions for all periods in a day other than AM-peak and PM-peak periods day for all stations $N$ that belong to road class $M$.

An example of two ATR stations is used to demonstrate the process to calculate peak period factors. The raw data for two ATR stations (1420 and 2400) between 1 November 1997 and 7 November 1997 are shown in Table 5.3. Both stations belong to the "urban interstate highway" road class.

The raw data were first screened based on the three rules described in "raw data screening." The screening process shows no apparent errors in these raw data: the values of two directions for both stations are different, there are no consecutive zero flows for both stations, and the directionality for any day for both directions do not exceed $80 \%$. Therefore, the data are ready for calculation of peak-period factors. 
The hourly volumes for both directions and both stations were summed up ( $\left.\sum_{D} \sum_{N \in M} V o l_{D L N J}\right)$ and entered into Table 5.4. For example, the value of 1491 for the period between midnight and 1:00AM on 1 November 1997 in Table 5.4 comes from the sum of the values 461 and 449 for the northbound and southbound flows at station 1420 and the values 253 and 328 for the northbound and southbound flows at station 2400 for the same period of time in Table 5.3.

The data were further classified into two day types: weekdays, which include Monday through Friday, and weekends, which include Saturday and Sunday. In Table 5.5, the shaded cells indicate weekend days; all other cells are weekdays. For each day type, the AM-peak period is defined as the three consecutive hours that have the maximum sum of three consecutive hourly volumes in both directions between midnight and noon. That is, for each group of road class $M$ and day type $L$, define $I A M_{M L} \equiv(A M \text { peak start time })_{M L}$

$$
\text { where } \sum_{D} \sum_{J=I A M_{M L}}^{I A M_{M L}+2} \sum_{N \in M} V o l_{D L N J}>\sum_{D} \sum_{J=J_{1}}^{J_{1}+2} \sum_{N \in M} V o l_{D L N J}, I A M_{M L}, J_{l} \in\{0,1, \ldots, 9\}, I A M_{M L} \neq J_{I}
$$

By applying the above rule, the sum of volumes in both directions between 9:00AM and 12:00PM $\left(\sum_{J=9}^{12} \sum_{D} \sum_{N \in M} V_{o l} l_{D L N J}\right)$ has the maximum value of 24,858 . Therefore, the AMpeak period for "weekend" is the period between 9:00AM and 12:00PM. Similarly, the AM-peak period for "weekday" is the period between 7:00AM and 10:00AM. The AMpeak periods were shown with dashed border lines in Table 5.5.

The PM-peak period for each day type is defined as the three consecutive hours with the maximum sum of three consecutive hourly volumes in both directions between noon and midnight. That is, define $I P M_{M L}=(P M \text { peak start time })_{M L}$ 
where $\sum_{D} \sum_{J=I P M_{M L}}^{I P M_{M L}+2} \sum_{N \in M} V o l_{D L N J}>\sum_{D} \sum_{J=J_{2}}^{J_{2}+2} \sum_{N \in M} V o l_{D L N J}, I P M_{N L}, J_{2} \in\{12,13, \ldots, 21\}, I_{2} \neq J_{2}$

By applying the above rule, both the PM-peak periods for weekends and weekdays are the periods between 3:00PM and 6:00PM. The PM-peak periods are shown with wider border lines in Table 5.5 .

Off-peak periods for each day type are defined as the periods other than AM-peak and PM-peak periods, that is:

- From midnight to the start time of AM-peak periods;

- From the end of AM-peak periods to the start time of PM-peak periods;

- From the end of PM-peak periods to midnight.

The PM-peak periods are shown with double solid lines in Table 5.5.

As described previously, the average peak period factor for road class $M$ and day type $L$ is defined as follows:

(AM peak period factor) $_{M L}=\frac{\frac{1}{3} \sum_{D} \sum_{N \in M} \sum_{J=I A M_{M L}}^{L A M M_{M L}+2} V l_{D L N J}}{\sum_{D} \sum_{N \in M} \sum_{J=0}^{23} V o l_{D L N J}}$

(PM peak period factor) $)_{M L} \equiv \frac{\frac{1}{3} \sum_{D} \sum_{N \in M} \sum_{J=I P M_{M L}}^{I P M_{M L}+2} V o l_{D L N J}}{\sum_{D} \sum_{N \in M} \sum_{J=0}^{23} V o l_{D L N J}}$

(Off peak period factor $)_{M L} \equiv \frac{\frac{1}{18} \sum_{D} \sum_{N \in M}\left(\sum_{J=0}^{I A M_{M L}-1} V o l_{D L N J}+\sum_{J=I A M_{M L}+3}^{I P M_{M L}-1} V o l_{D L N J}+\sum_{J=I P M_{M L}+3}^{23} V o l_{D L N J}\right)}{\sum_{D} \sum_{N \in M} \sum_{J=0}^{23} V o l_{D L N J}}$ 
The numerator of the AM-peak factor for weekends is 24,858 (sum of volumes between 9:00AM and noon in Table 5.5) divided by 3, and the denominator of peak period factors for weekends is 148,261 in Table 5.5. Therefore, the AM-peak period factor for weekends is $0.056\left(=\frac{\frac{1}{3} \cdot 24,858}{148,261}.\right)$ Similarly, AM-peak period factor for weekdays is 0.061 , the PM-peak period factors for weekends and weekdays are 0.074 and 0.078 , and the Off-peak period factors for weekends and weekdays are 0.034 and 0.032 .

The calculation of peak period factors above is based on the sum of all ATR stations belonging to the same road class. Not all stations belonging to the same road class have the same three hours in the AM or PM peak period pattern, but we need to establish a standard three-hour peak period to apply to non-ATR links for which only AADT values are available. Table 5.6 shows that, for our two-station example, both weekday peak periods are the same and the weekend AM peaks also cover the same three hours. However, the weekend PM peaks differ at station 1420, the PM peak starts at 2:00PM on weekend; it starts at 3:00PM at station 2400 .

The above results support the approach using the sum of volumes for all ATR stations belonging to the same road class. This approach gives greater weight to the ATR stations with higher volumes, which are more likely to cause delays.

C. Directionality estimation

Directionality varies for different highways and different time frames. Because of this characteristic of directionality, the expected time and effort to be spent on estimating directionality for different highways and different time frames is very large. To simplify the directionality estimation process while still being able to predict the major delays for 
all highways within Indiana, the HTIS adopts a safe-side approach by applying the higher directionality to both directions for all highway links. The directionality for different day types and different road classes for a peak period is determined by dividing the sum of the larger volumes in the two directions for each hour within the peak period by the sum of volumes in both directions for each hour within the peak period.

Define $\operatorname{DirVol}_{L N J}$ as the larger volumes between two directions for day type $L$, station $N$, at hour $J$. That is,

$$
\operatorname{DirVol}_{L N J}=\operatorname{Max}\left(\operatorname{Vol}_{D_{1} L N J}, \operatorname{Vol}_{D_{2} L N J}\right)
$$

The directionality for road class $M$ for day type $L$ is defined as follows:

$$
\begin{aligned}
& \text { (AM-peak Directionality) }{ }_{M L} \equiv 100 \% \times \frac{\sum_{N \in M J=I A M_{M L}}^{I A M_{M M}+2} \operatorname{DirVol}_{L N J}}{\sum \sum \sum^{I A M_{M L}+2}}, D \in\left\{D_{1}, D_{2}\right\} \\
& \sum_{D} \sum_{N \in M} \sum_{J=I A M}^{I A M L} V_{M L} l_{D L N J} \\
& \left(P M \text {-peak Directionality) }{ }_{M L} \equiv 100 \% \times \frac{\sum_{N \in M J=I P M_{M L}}^{I P M_{M L}+2} \operatorname{DiVol}_{L N J}}{\sum \sum \sum^{I P M_{M L}+2}}, D \in\left\{D_{1}, D_{2}\right\}\right. \\
& \sum_{D} \sum_{N \in M} \sum_{J=I P M_{M L}}^{I P M_{M L}+2} V o l_{D L N J}
\end{aligned}
$$

(Off-peak Directionality) $_{M L} \equiv$

$$
100 \% \times \frac{\sum_{N \in M}\left(\sum_{J=0}^{I A M_{M L}-1} \operatorname{DirVol}_{L N J}+\sum_{J=I A M_{M L}+3}^{I P M_{M M}-1} \operatorname{DiV}_{L N}+\sum_{J=I P M_{M L}+3}^{23} \operatorname{DirVol}_{L N J}\right)}{\sum_{D} \sum_{N \in M}\left(\sum_{J=0}^{I A M_{M L}-1} V o l_{L N J}+\sum_{J=I A M_{M L}+3}^{I P M_{M L}-1} V o l_{L N J}+\sum_{J=I P M_{M L}+3}^{23} V o l_{L N J}\right)}, D \in\left\{D_{1}, D_{2}\right\}
$$

The same example shown in Table 5.3 is used to explain the calculation process for directionality estimation. The sums of volumes for all days belonging to the same day type for each direction and each ATR station $\left(V_{0} l_{D L N J}\right)$ are shown in Table 5.7. For 
example, the value of 831 for the period between midnight and 1:00AM for northbound weekend day type at station 1420 in Table 5.7 comes from the sum of the value of 461 and 370 on Saturday, 1 November and Sunday, 2 November 1997 for that time period Table 5.3.

The larger volumes between two directions ( $\left.\operatorname{DirVol}_{L N J}\right)$ were then selected (Table 5.8a). For instance, the value of 832 for the period between midnight and 1:00AM for weekends at station 1420 comes from the larger of 831 for southbound volumes and 832 for northbound volumes.

Next, the sums of the larger volumes between two directions for all stations belonging to the same road class $\left(\sum_{N \in M} \operatorname{DirVol}_{L N J}\right)$ were calculated (Table 5.9). For instance, the value of 1,385 for the period between midnight and 1:00AM for weekends for both stations comes from the sum of the value of 832 and 553 for the same time period for stations 1420 and 2400 in Table 5.8 .

Finally, the directionalities for different day types were estimated (Table 5.10). The numerators of AM-peak and PM-peak directionalities $\left(\sum_{N \in M J=I A M_{M L}}^{L A M_{M M}+2} \operatorname{DirVol}_{L N J}\right.$ and $\left.\sum_{N \in M J=I P M_{M L}}^{I P M_{M L}+2} D_{i r} V o l_{L N J}\right)$ represent the sum of the larger volumes between two directions for all stations belonging to the same road class during the peak periods. The numerators of AM-peak directionalities for weekends and weekdays are shown with dashed border lines in Table 5.10 (13,569 and 50,863). The numerators of PM-peak directionalities for weekends and weekdays are shown with solid border lines $(17,852$ and 61,945$)$. The numerators for off-peak directionalities are the sum of the larger volumes between two 
directions for all periods except AM-peak and PM-peak periods. The values are shown with double border lines in Table 5.10 (47,599 for weekends and 149,334 for weekdays).

The denominators of peak period directionalities represent the sum of volumes for both directions for all stations belonging to the same road class during the peak periods. The denominators of AM-peak directionalities for weekends and weekdays are 24,858 and 86,507 in Table 5.5, and the denominators of PM-peak directionalities for weekends and weekdays are 33,084 and 111,195 . The denominators of off-peak directionalities are the sum of volumes for both directions for all stations belonging to the same road class for all periods except AM-peak and PM-peak periods. The values of the denominators of off-peak directionalities for weekends and weekdays are 90,319 and 277,396.

The peak period directionalities can then be calculated. The AM-peak directionality for weekends is $54.6 \%\left(=\frac{13,569}{24,858} \times 100 \%\right.$.) Similarly, the AM-peak directionality for weekdays is $58.8 \%$, the PM-peak directionalities for weekends and weekdays are $54.0 \%$ and $55.7 \%$, and the off-peak directionalities for weekends and weekdays are $52.7 \%$ and $53.8 \%$.

D. Growth factor

Annual growth factors quantify the rate at which AADT increases from one year to the next. Annual growth factors are used to convert AADTs in different years to the same base year. In the following contents, three different methods for estimating growth factors are shown - INDOT method, TMG method, and the one used in the HTIS.

(1) INDOT method 
The annual growth factors used by INDOT were obtained from ATR data. The first step in the estimation processes is to calculate the monthly average daily traffic (MADT) for each ATR station for each month in a specific year. Define $V o l_{D J N T P Y}$ as the hourly volume of direction $D$ at station $N$ during hour $J$ on date $T$ in month $P$ and year $Y$, and $M A D T_{N P Y}$ as the MADT for station $N$ in month $P$ and year $Y$. Then, $M A D T_{N P Y}$ is calculated based on the following equation:

$$
M A D T_{N P Y}=\frac{\sum_{T} \sum_{D} \sum_{J=0}^{23} V o l_{D J N T P Y}}{m_{P Y}}
$$

where $m_{M Y}$ is the number of days in month $P$ and year $Y$.

Next, the AADT for the specific year for each station is calculated. Because malfunctions at ATR stations are not unusual, not all of the data for each station for each month of the specific year are available. Because estimation of annual growth factors involve data from two consecutive years, the calculations of AADTs of these two consecutive years should be based on the same months of data. Therefore, the AADT of each station is defined as the average of MADTs for all the common available months within the two consecutive years. For example, a particular ATR station did not function well in February and April 1996 and in February and August 1997. The MADTs of February, April and August in both 1996 and 1997 are discarded when calculating the AADTs of 1996 and 1997 for the estimation of the annual growth factor from 1996 to 1997. That is, the AADTs for both 1996 and 1997 are based on the same 9 months (excluding February, April and August). Define $A A D T_{\mathrm{NY}}$ as the AADT of station $N$ in year $Y$. It is calculated based on the following equation: 


$$
A A D T_{N Y}=\frac{\sum_{P} M A D T_{N P Y}}{q}
$$

The $q$ in the above equation is the number of months in year $Y$ of which the data are available for the two consecutive years from which the growth rates are intended to be calculated. For the above example, $q$ equals 12 months less than 3 months (February, April and August) for both the years 1996 and 1997.

The third step is to classify the ATR data into one of five categories based on functional classes: urban interstate highways, urban others, rural interstate highways, rural principal and minor arterials, and rural major and minor collectors and locals. For each category, the AADT of that category is calculated. Define $A A D T_{M Y}$ as the AADT of category $M$ in year $Y$. It is calculated based on the following equation:

$$
A A D T_{M Y}=\frac{\sum_{N \in M} A A D T_{N Y}}{n_{M Y}}
$$

where $n_{M Y}$ is the number of stations belonging to category $M$ in year $Y$.

Finally, the growth factors of different categories are obtained. Define $G_{M Y}$ as the growth factor for the conversions of AADT from year $Y-1$ to year $Y$. It is calculated based on the following equation:

$$
G_{M Y}=\frac{A A D T_{M Y}}{A A D T_{M(Y-1)}}
$$

The annual growth factors by functional classes from 1990 to 1999 adopted by INDOT are shown in Table 5.11. These values were applied to the data from county flow map to convert them to AADTs with a base year of 1995. The AADTs for 1995 are 
available in the TransCAD network file as in the field name "ADTVOL" as shown in Figure 3.3.

(2) TMG method (FHWA, 1995)

The basic TMG requirements for the estimations of annual growth factors are complete ATR data for the two consecutive years. The problem of incomplete ATR data is not addressed in TMG. For those stations that recorded complete data for the entire year, the AADT for each is simply the average of MADTs. That is,

$$
A A D T_{N Y}=\frac{1}{12} \sum_{P} M A D T_{N P Y}
$$

where $M A D T_{N P Y}$ is the $M A D T$ for station $N$ in month $P$ in year $Y$.

Next, the growth factors for each station are defined as the ratios of AADTs of each station for the corresponding two consecutive years.

Define the annual growth factor of station $N$ in year $Y$ as $G_{N Y}$.

$$
G_{N Y}=\frac{A A D T_{N Y}}{A A D T_{N(Y-1)}}
$$

where $A A D T_{N Y}=$ annual average daily traffic (AADT) at station $N$ for year $Y$, and

$$
A A D T_{N(Y-1)}=\text { AADT at station } N \text { for year } Y-1 \text {. }
$$

Finally, the growth factors for each road class is calculated based on the average of the growth factors for all ATR stations belonging to the same road class.

The annual growth factor $G_{M}$ for road class $M$ in year $Y$ is defined as

$$
G_{M Y}=\frac{\sum_{N \in M} G_{N Y}}{n_{M Y}}
$$


where $n_{M Y}=$ number of stations belonging to road class $M$ and functioning well in year $Y$.

(3) HTIS method

The method of estimating growth factors used in the HTIS is modified based on TMG method. The TMG method cannot deal with the problem of incomplete ATR data for the two consecutive years due to malfunctions at ATR stations. To overcome this problem, seasonal adjustment provided by INDOT were applied to those ATR stations that did not function well at certain times during a year. The seasonal adjustment factors (Table 5.12) are classified, based on different road classes, by years and months.

Define the sum of the valid MADTs of station $N$ for month set $S$ in year $Y$ as SubSum $_{M A D T, Y}$ and SF as the seasonal adjustment factor. The month set $S$ is the incomplete ATR data consisting of any combination of the number of months between one and eleven. Also define the sum of the MADTs of station $N$ for the entire year $Y$ as Sum $_{M A D T,}$, the sum of the SFs for the months in month set $\mathrm{S}$ in year $Y$ as $\operatorname{SubSum}_{S F, Y}$, and the sum of the SFs for the entire year $Y$ as $\operatorname{Sum}_{S F, Y}$. The sum of all MADTs in year $Y$ can be estimated by assuming that the percentage of the sum of the valid MADTs to the sum of all MADTs in year $Y$ is the same as the percentage of the sum of the corresponding seasonal factors to the sum of all seasonal factors in year $Y$. That is:

$$
\begin{aligned}
& \frac{\operatorname{SubSum}_{M A D T, Y}}{\operatorname{Sum}_{M A D T, Y}}=\frac{\operatorname{SubSum}_{S F, Y}}{\operatorname{Sum}_{S F, Y}} \\
& \Rightarrow \operatorname{Sum}_{A D T, Y}=\frac{\operatorname{SubSum}_{M A D T, Y} \cdot \operatorname{Sum}_{S F, Y}}{\operatorname{SubSum}_{S F, Y}}
\end{aligned}
$$

The AADT for station $N$ in year $Y$ is the average of the sum of all MADTs in the year. 


$$
A A D T_{N Y}=\frac{\operatorname{Sum}_{M A D T, Y}}{12}=\frac{\operatorname{SubSum}_{M A D T, Y} \cdot \operatorname{Sum}_{S F, Y}}{12 \cdot \operatorname{SubSum}_{S F, Y}}
$$

where $A A D T_{N Y}$ is the AADT for station $N$ in year $Y$.

For example, ATR station 1120 on a rural interstate highway recorded valid data throughout February, March, and May and had malfunctions in all other months in 1997. The MADTs of station 1120 in February, March and May 1997 were 28,000, 29,000, and 27,500. From Table 5.12, the SFs for road class of rural interstate highways in February, March, and May, 1997 are 1.042, 0.951 and 1.014, and the sum of the SFs for the same road class in 1997 is $12.321(=1.128+1.042+0.951+1.117+1.014+1.007+0.922+$ $0.938+1.036+1.011+1.032+1.124)$. The AADT for station 1120 in 1997 is calculated as follows:

$$
A A D T_{1120,1997}=\frac{1}{12} \times \frac{(28,000+29,000+27,500) \cdot 12.321}{1.042+0.951+1.014}=28853
$$

In addition to the problem of malfunctions at ATR station, the TMG cannot deal with missing ATR data. For example, the ATR data for January 1997 are not available from INDOT. In order to obtain the MADT of the missing data, the HTIS assumes that the growth rates for each station from 1997 to 1998 and are the same as the growth rates from 1996 to 1997 . The MADT for January 1997 can be estimated based on the MADTs for January 1996 and January 1998. That is,

$$
\frac{M A D T_{N, 1 / 1997}}{M A D T_{N, 1 / 1996}}=\frac{M A D T_{N, 1 / 1998}}{M A D T_{N, 1 / 1997}}
$$

where $M A D T_{N, 1 / 1996}=$ monthly average daily traffic (MADT) for station $N$ in January

$$
\begin{gathered}
1996, \text { and } \\
M A D T_{N, 1 / 1997}=\text { MADT for station } N \text { in January 1997, and }
\end{gathered}
$$




$$
\begin{aligned}
M A D T_{N, 1 / 1998} & =\text { MADT for station } N \text { in January } 1998 . \\
\Rightarrow M A D T_{N, 1 / 1997} & =\sqrt{M A D T_{N, 1 / 1996} \cdot M A D T_{N, 1 / 1998}}
\end{aligned}
$$

Moreover, there exists a problem of matching old ATR stations with new ones (Figures 3.1a and 3.1b). More than thirty percent of the ATR stations were relocated in 1999. Some stations were moved away from the original locations. The longest distance between old and new ATR location is 7 miles. Some stations were newly located and do not have corresponding old stations. Some of the old stations were even removed completely. This makes it hard to compare the trend in traffic patterns before and after 1999 for those relocated ATR stations.

For the purpose of comparing the estimation results from the processes suggested by the TMG with the ones adopted by INDOT, the HTIS calculates the annual growth factors from 1997 to 1998 following the TMG procedures. The problems of incomplete ATR data and missing ATR data are overcome by using the method proposed by the HTIS. The results are shown in Table 5.13. The road classification used by INDOT is different from the one used in the HTIS. The road classification adopted in the HTIS is based on the TMG - "urban interstate", "other urban", "rural interstate", and "other rural" highways. INDOT further classifies the "other rural" highways into two categories "rural arterials" and "rural collectors." The average growth factor for the "rural arterials" and "rural collectors" from INDOT is compared with the growth factor for the "other rural" highways in the HTIS.

In Table 5.13, all of the growth factors based on the TMG procedure are higher than the ones adopted by INDOT, except for the "rural other highways" road class. In order to 
consistent with the calculations of the base-year traffic demands (AADTs of 1995) in TransCAD network file, the values adopted by INDOT are used in the HTIS.

Because the growth factors from 1999 to the forecast year are not yet available from INDOT, the average growth rate from 1995 to 1999 is used as the growth rate from 1999 to the forecast year. The total growth rates of AADTs from base year 1995 to year $Y$ is defined as the product of annual growth factors from 1995 to 1999 and the power of $(Y$ 1999) to the average value of annual growth factors from 1995 to 1999 . For example, the total growth rate of AADTs for the road class "urban interstate highways" is the product of the annual growth factors from 1995 to $1999(1.047,1.066,1.038$ and 1.038) and the power of 2 applied to the average values of the annual growth factors from 1995 to 1999 , which is equal to 1.0473 . That is:

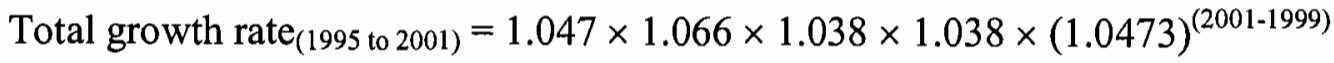

$$
\approx 1.319
$$

E. Link volume forecasting

Link volume forecasting is a process to obtain average link hourly volumes for three different periods in a day for different day types and road classes based on base-year traffic demands. The base-year traffic demands in the HTIS are defined as the daily volumes in the TransCAD network file, which are converted from the county flow map. The average hourly volumes in three different periods in a day, AM-peak, PM-peak, and off-peak, for each link for different day types, are calculated by applying the average peak period factors, directionality factors, and growth factors to the daily volumes in the TransCAD network file. 
The directionality factors are applied to both directions of each link. Because the directional distribution for each link is related to the geographic location of the link, the true direction distribution cannot be decided based on the ATR data. The hourly volumes in both directions are applied with directionality factors as conservative estimates.

Define $A D T_{X, 1995}$ as the daily volume of link $X$ in 1995 , and $V o l_{L X J, P / Y}$ as the hourly volume in either direction of the link belonging to road class $M$ for day type $L$ during hour $J$ in month $\mathrm{P}$ and year $Y$.

$\operatorname{Vol}_{L X, P / Y} \equiv A D T_{X, 1995} \times(\text { peak factor })_{M L J P} \times(\text { directionality })_{M L J P} \times$ total growth rate $\left.{ }_{(1995 \text { to }}\right)$ where (peak factor) $)_{M L J P}$ and (directionality) ${ }_{M L J P}$ are decided by hour $J$ in month $P$

$$
(\text { peak factor })_{M L P P}=\left\{\begin{array}{c}
(A M \text {-peak period factor })_{M L} J \in\left\{I A M_{M L}, I A M_{M L}+1, I A M_{M L}+2\right\} \\
(\text { PM-peak period factor })_{M L}, J \in\left\{I P M_{M L} I P M_{M L}+1, I P M_{M L}+2\right\} \\
(\text { Off-peak period factor })_{M L}, J \in\left\{0, I, \ldots, I A M_{M L}-1, I A M_{M L}+3, I A M_{M L}+4, \ldots,\right. \\
\left.I P M_{M L}-1, I P M_{M L}+3, I P M_{M L}+4, \ldots, 23\right\}
\end{array}\right.
$$

in which (AM-peak period factor $)_{M L},(P M \text {-peak period factor })_{M L}$, and (Off-peak period actor) $)_{M L}$ are defined in "Average peak period factors estimation" earlier this section.

$$
(\text { directionality })_{M L P P}=\left\{\begin{array}{r}
(A M \text {-peak directionality })_{M L} J \in\left\{I A M_{M L}, I A M_{M L}+1, I A M_{M L}+2\right\} \\
(P M \text {-peak directionality })_{M L} J \in\left\{I P M_{M L} I P M_{M L}+1, I P M_{M L}+2\right\} \\
(\text { Off-peak directionality })_{M L} J \in\left\{0,1, \ldots, I A M_{M L}-1, I A M_{M L}+3, I A M_{M L}+4,\right. \\
\left.\ldots, I P M_{M L}-1, I P M_{M L}+3, I P M_{M L}+4, \ldots, 23\right\}
\end{array}\right.
$$

in which (AM-peak directionality) $)_{M L},(P M-\text {-peak directionality) })_{M L}$, and (Off-peak

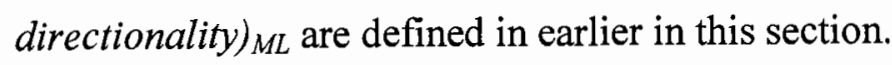


For example, the ADT on a particular rural interstate highway in the base year of 1995 is 30,000 . One wants to estimate the hourly volume in either direction on the link from 9:00AM to 10:00AM on a weekday in May 1999. The AM-peak period for weekday and road class "rural interstate highway" in May is between 9:00AM and 12:00PM. Suppose the AM-peak factor for weekday and on rural interstate highways in May is 0.046 , the AM-peak directionality for the same group of data is $60 \%$, and the total growth rate for rural interstate highways from 1995 to 1999 is $1.169(=1.021 \times 1.070 \times$ $1.036 \times 1.033$ ), the hourly volume in either direction of the link from 9:00AM to 10:00AM on a weekday in May 1999 is:

$$
\text { Vol }_{L=\text { weekday, }, X \in \text { rural interstate, } J=9,5 / 1999}=30,000 \times 0.046 \times 60 \% \times 1.169=968(\mathrm{vph})
$$

\subsection{O-D Estimation}

The O-D estimation process is one of the major processes in the "complete information" analysis (Figure 5.1). The function of O-D estimation in the HTIS is to obtain the demands for each O-D pair during the period of time being forecasted. The estimated O-D matrix will be loaded onto the state highway network to simulate the traffic conditions that represent the "complete information" scenario. These are the lower bound of link volumes. As explained at the beginning of this chapter, drivers are assumed to keep their normal departure time, despite the existence of workzones. Under the assumption that highway users may only change their routes and not their departure times, the demands for each O-D pair at a specific time are assumed to be the same for both the "no information" and the "complete information" scenarios. Therefore, the O-D table for the "complete information" scenario is assumed to be the same as for the "no 
information" scenario. The O-D table for the "no information" scenario can be estimated from O-D estimation based on the "no information" link volumes. The estimated O-D table is then assigned to the network with workzone constraints to produce a flow pattern under the "complete information" scenario.

The O-D estimation model used in the HTIS is the built-in function in TransCAD based on Nielsen's study (1993). Nielsen proposed a new method of estimating trip matrices called Single Path Matrix Estimation (SPME) to solve the problem of data inconsistency among traffic counts. The SPME model treats traffic counts as stochastic variables, that is, traffic counts that are not error-free. Because traffic counts are not fixed in the SPME model, they can be adjusted to meet the conservation of flow principle. The problems caused by data inconsistency can be reduced. In addition, the SPME model converges faster than the entropy maximizing (EM) and information minimizing (IM) models. The SPME model uses a bi-level programming algorithm that can separate matrix estimation from traffic assignment. It is only necessary to perform a traffic assignment each time all elements in the O-D matrix have been modified. The estimation process stops when the squared error between traffic counts and estimated flows is reduced to an acceptable limit.

The inputs for performing O-D estimation using TransCAD include observed link counts, an a priori matrix and user-specified traffic assignment methods (Caliper Inc., 1999).

\section{A. Observed link volumes}

The O-D estimation process in TransCAD uses observed link counts as constraints to produce an O-D matrix that is consistent with the observed link counts. In the HTIS, the 
"observed link counts" are the "no information" link volumes obtained from the traffic volume forecasting done in Section 5.2. As explained at the beginning of this section, the demands for each O-D pair for the "no information" and the "complete information" scenarios remain the same under the assumption that travelers will have the same trip demands at the same time period, whether they are aware of workzones or not. Therefore, the "no information" link volumes, which simulate the traffic conditions for the "no information" scenario, are used to perform O-D estimation to obtain the demands for each O-D pair for the "no information" scenario. The demands for each O-D pair for the "complete information" scenario are assumed to be the same as for the "no information" scenario.

By using the "no information" link volumes for different time periods and day types in a specific month as input, the demands for each O-D pair (O-D table) for the corresponding time periods and day types in the month can be obtained.

B. A priori matrix

The priori matrix provides initial trip matrix values for O-D estimation. The time periods for a priori matrix should be consistent with all other inputs for O-D estimation. Therefore, the a priori matrix for the HTIS should be in hourly format, as are the observed link volumes, which are the "no information" link volumes. The initial O-D tables available from INDOT are the daily, AM-peak, PM-peak and off-peak O-D tables (see Section 3.3). However, none of these O-D tables are in hourly format. In addition, the AM-peak, PM-peak and off-peak O-D tables contain only internal-internal trips. The only available initial O-D table that contains all internal-internal, internal-external and 
external-external trips is the daily O-D table. The daily O-D table needs to be converted to hourly O-D table before it can be used for O-D estimation in the HTIS.

A more detailed way to convert INDOT's daily O-D table into hourly format is to take trip purposes of trips into consideration. Cambridge Systematics Inc. (1998) used time-of-day factors for different trip purposes obtained from the household travel survey data to adjust the daily O-D table to the AM-peak, PM-peak and off-peak O-D tables. However, the household travel survey data do not provide enough information for estimating the time-of-day factors for internal-external trips. Therefore, the approach using time-of-day factors for different trip purposes cannot be used in the HTIS.

The HTIS adopts a simplified approach to estimate the initial hourly O-D tables for different day types in a specific month. First, the average growth factor of all road classes from the base year of INDOT's daily O-D table (1995) to the forecast year is applied to INDOT's daily O-D table to obtain a daily O-D table for the forecast year. Then, the average peak period factors of all road classes for different day types in the specific month are applied to all cells in the daily O-D table for the forecast year. The initial hourly O-D tables for each day type in the specific month of the forecast year can therefore be roughly estimated. The calculations for the growth factors of different road classes and the peak period factors of different road classes and day types are shown in Section 5.2.

C. Traffic assignment methods

Traffic assignment methods are required to perform the O-D estimation process in TransCAD. As explained previously in this section, the O-D estimation process in TransCAD is based on Nielsen's study (1993), which is a bi-level process that switches 
back and forth between traffic assignment and O-D estimation. The choice of traffic assignment method can affect the results of the O-D estimation. The available options for traffic assignment in TransCAD are "user equilibrium", "stochastic user equilibrium", "system optimization", "capacity restraint", "incremental" and "all or nothing." Among these options, the options "user equilibrium" and "stochastic user equilibrium" are suggested in the TransCAD users' manual if no special requirements are needed. The evaluation of these two options is shown in Section 5.4. According to the evaluation results, the option "user equilibrium" for traffic assignment is used in the HTIS.

The output from each O-D estimation process is an estimated O-D table, which represents the demands for each O-D pair during the time corresponding to that of the inputs for O-D estimation. For example, if the "no information" link volumes for the AM-peak period during a typical weekday in May 2000 are used for O-D estimation, the output O-D table is the demand for each O-D pair for the same time. The output O-D table is then assigned to the network with workzone constraints to obtain the "complete information" link volumes, the lower bounds of link volumes. The assignment process is explained in Section 5.4 .

The O-D estimation process is repeated for different sets of inputs. The observed link volumes vary for each peak period and day type, while the a priori matrices vary for each day type. Therefore, the O-D estimation process is performed for each peak period and day type. The number of combinations required for performing O-D estimation for a specific month is the same as the number of different sets of observed link volumes (the "no information" link volumes) in the month. 


\section{$\underline{5.4 \text { Traffic Assignment }}$}

The function of traffic assignment in the HTIS is to estimate the "complete information" link volumes, which form the lower bounds for link volumes. In the "complete information" scenario, it is assumed that all travelers have complete knowledge about workzone locations. Travelers will make decisions based on the complete information and choose the best route on which the expected delay is minimized. The route choice behavior in the "complete information" scenario can be simulated by selecting an appropriate traffic assignment method and assigning the O-D tables obtained from O-D estimation in Section 5.3 to the network with workzone constraints. The resulting link volumes are the "complete information" link volumes for the same time period as the input O-D tables.

Traffic assignment methods can be divided into static and dynamic models, depending on the considerations of time frames. Static assignment models use larger time frames, such as a day or a few hours, in which flow rates and travel times can be considered as constant over time. Dynamic assignment models, on the other hand, assign traffic demands to much smaller time frames. The trips may not be completed within the time frame. Therefore, vehicles travel time needs to be considered. Dynamic assignment models are generally more realistic in simulating route choices behavior than static assignment models. Reviews of static and dynamic assignment models can be seen in Ortuzar and Willumsen's book (1996) and Pavlis and Papageorgiou's paper (1999).

Static assignment models are chosen for use in the HTIS for the following reason. The HTIS is a pre-trip travel information system providing information about the impacts of scheduled events such as road construction on traffic conditions. The durations of 
scheduled events (such as road construction) are normally much longer than real-time events (such as incidents). Because of these longer durations, the traffic at workzones after an initial transition period reaches an equilibrium condition for each time period that permits use of a static traffic assignment method.

The most commonly used static models include all-or-nothing (AON), stochastic, user equilibrium (UE), stochastic user equilibrium (SUE) and system optimum (SO) (Ortuzar and Willumsen, 1996). Among these static models, UE and SUE are the most suitable for use in the HTIS, because the traffic conditions depicted in these two methods are similar to the "complete information" scenario, in which each traveler chooses the route with minimum cost. Both UE and SUE assume each traveler chooses the route with minimum cost. The major difference between UE and SUE is that in SUE each traveler defines "travel costs" individually instead of using a single definition for all travelers. These two models are further evaluated in the following paragraphs.

The evaluation of UE and SUE assignment models was performed using TransCAD. The required inputs for both assignment models in TransCAD include an O-D matrix and a network file, which contains the data about link travel time and link capacity (Caliper, 1999). The model settings for UE include:

- Number of iterations: maximum number of iterations to be performed. The default value is 20 .

- Convergence criterion: If the maximum absolute change in all the link flows between consecutive iterations is less than this value, the assignment process stops. The default value is 0.5 . 
- $\alpha$ and $\beta$ : the parameters in the BPR functions. The default values are 0.15 and 4 , respectively.

SUE requires two more settings in addition to the settings for UE:

- Error term distribution function: The default distribution is Normal.

- Percentage error for the error term: The default value is 5 .

To determine a suitable assignment model for use in the HTIS, the stability of the assignment results were evaluated by varying the number of iterations used in addition to the closeness of the assigned traffic to the observed link volumes. Whichever converges faster and assigns traffic closer to the observed link volumes is preferable.

The O-D matrix used for evaluation is the daily O-D table obtained from INDOT. Although the time frame in the HTIS is set to be an hour, the hourly O-D matrices are not used because they are estimated from the daily O-D table and the errors caused by the additional estimation process might lead to biased results. As explained in Section 3.3, the daily O-D table was generated from O-D estimation using the seed table and ADTs in the network file applied with directional distribution as "traffic counts" (Cambridge Systematics Inc, 1998). A directional distribution of 50\% was used to obtain directional link volumes from the ADTs in the network file. Hypothetically, in an error-free scenario, when the daily O-D table is assigned back to the network, the assigned link volumes should match the ADTs in the network file applied with directional distribution, which are 0.5 ADTs. However, the directional link volumes obtained by applying $50 \%$ directionality are not the "real" traffic counts. If these values are compared with the assigned link volumes, the errors caused by the hypothetical directionality may bias the results. Therefore, the sum of assigned link volumes in two directions is compared with 
the sum of "traffic counts" in two directions, which are the standard ADTs. By assuming that the errors caused in the daily table generated from O-D estimation have the same effects on different assignment models, the better assignment model is the one that produces the lower total difference between the sum of assigned link volumes in both directions and the ADTs in the network file.

Define " $A$ " as the ADTs and " $B$ " as the sum of assigned link volumes in two directions. Three fitting criteria are defined for comparison of the sum of assigned link volumes in both directions and the ADTs:

(1) $\Sigma|\mathrm{A}-\mathrm{B}|$;

(2) $\Sigma\left(|\mathrm{A}-\mathrm{B}|^{2} / \mathrm{A}\right)$;

(3) Number of zero-flow links.

The first criterion gives equal weight to all links, while the second criterion known as Chi-squared form, gives greater weight to links with lower ADTs because the ADTs are used as the denominator in this criterion (Ortuzar and Willumsen, 1996). The third criterion verifies if the stochastic characteristic in SUE helps to assign traffic in a more realistic way. Because different weighting principles may result in different conclusions, all three criteria are used in the HTIS. The lower values of the criteria, the better the assignment model is.

The results of evaluation are shown in Table 5.14. In Table 5.14, the values of the first two criteria for UE are the same for 20 and 25 iterations, which means the assigned results from UE are stable after 20 iterations. The values of the first two criteria for SUE, however, gradually decrease as the number of iterations increases. The gradually decreasing trend still remains at 200 iterations, which means the assigned results for SUE 
are still not stable at 200 iterations. The values of the first two criteria for UE are lower than for SUE, which indicates UE is more preferable. The third criterion shows SUE produces less number of zero-flow links than UE. Although this criterion indicates that SUE assigns traffic in a more realistic way than UE, the problems of stability and less close to ADTs using SUE make it less preferable. The UE model is chosen for use in the HTIS.

The output of the traffic assignment process is the "complete information" link flow pattern for the same period as the input O-D table. The "complete information" link volumes and the "no information" link volumes, which are from traffic volume forecasting, form the lower bound and the upper bound for link volumes. These two volumes are used as inputs for delay estimation, which is explained in Section 5.5.

The traffic assignment process needs to be repeated for different sets of inputs. The input O-D table changes for each peak period and day type, while the link capacities in the input network file change whenever the lane closure status (close or reopen) of any projects changes. The number of combinations required for repeating the assignment process is the combination of different input O-D tables and different lane closure statuses during the same periods of time as the input O-D tables.

\section{$\underline{5.5 \text { Delay Estimation }}$}

The objective of delay estimation is to predict queueing delays as a function of link demands. As explained in Section 5.1, link demands may vary as the workzone ages. At the beginning of construction, few drivers may know about any lane closures that may exist. As a result, most drivers will attempt to follow the routes they normally travel. 
This situation is simulated as the "no information" scenario. As the construction continues, more drivers become aware of the reduced roadway capacity. Some of the informed drivers may divert to alternate routes to reduce their delays. After a certain period of time, the number of drivers who know about the road construction reaches a maximum value. This value depends on the proportion of drivers who use this route on a regular basis and on the extent to which less frequent users can be informed by a road information service. Most of these informed drivers make route choice decisions to minimize delays. The situation in which all drivers know about the construction zone is simulated as the "complete information" scenario. The challenge is to find the "equilibrium" point between the "no information" and "complete information" scenarios.

The HTIS uses two steps to estimate queueing delays to account for the variation of link volumes: estimating upper and lower bounds for delay and refining delay estimates. Estimation of delay boundary determine queueing delays under the "no information" and "complete information" scenarios. The two values form the upper bound and lower bound on possible delays due to construction. (See Section 5.5.1.)

Workzone Delay Equilibrium Estimation (WDEE) attempts to find a new "steady state" for delay based on the level of traffic that will use the workzone link after its existence has become widely known. This is done by applying a relationship between link volumes and the age of the construction zone to the "no information" and the "complete information" link volumes. Even after the workzone has been in place for a long time, there will be non-local drivers who do not know about it. In addition, some local drivers may be willing to tolerate some extra delay on a known route that includes the workzone, rather than seeking new, unfamiliar routes. For these two reasons, 
workzone traffic flows will never reach the lower bound represented by the "complete information" scenario. The link volumes estimated by WDEE are then used to determine the queueing delays during construction periods. (See Section 5.5.2.)

\subsubsection{Estimation of Delay Boundary}

The boundary values for queueing delays caused by construction zones are determined by the link volumes found under the "no information" and the "complete information" scenarios. The delays under the "no information" scenario form the upper bound and the delays under the "complete information" scenario form the lower bound. The calculation of queueing delays is based on a queueing diagram approach. A typical queueing diagram is shown in Figure 5.12. In Figure 5.12, "A" represents the total queueing delay for all vehicles that enter the queue between time $T_{1}$ and time $T_{2}$. The average queueing delay per vehicle during that time period is the area "A" in Figure 5.12 divided by the number of vehicles entering the system during that time period. The unit for the average queueing delay per vehicle is "minutes." The average queueing length during a certain period of time is defined as the area " $\mathrm{B}$ " in Figure 5.12 divided by the time period. The unit for the average queueing length is "vehicles."

The basic input requirements for queueing analysis are the service rate and arrival rate.

\section{Service rate}

Service rates are the hourly capacities of the links under construction. Dudek and Richards (1981) and Jiang (1999) measured workzone capacities. However, the measured workzone capacities provided by these two studies do not agree. For a four- 
lane highway with two lanes closed, the workzone capacity based on Dudek and Richards' study is $1,340 \mathrm{vphpl}$, and the one based on Jiang's study is $1,466 \mathrm{vphpl}$. Memmott and Dudek (1982) introduced the concept of a "capacity estimate risk factor" (CERF) to reflect the variations of workzone capacities at different locations. The CERF is the probability that the estimated capacity will be less than or equal to the actual capacity (Figure 5.13). Memmott and Dudek used linear approximation to obtain the cumulative distributions of actual workzone capacity based on the survey results in Dudek and Richards' study. Because the cumulative distribution of actual workzone capacity is approximately linear, the probability distribution is uniform. When CERF equals $0 \%$, the probability that the estimated capacity is less than or equal to the actual capacity is 0 . When CERF equals $100 \%$, the probability that the estimated capacity is less than or equal to the actual capacity is $100 \%$. For the same lane closure type as in the above example (a four-lane highway with two lanes closed), the estimated capacity is $1460-(2.13 \times \mathrm{CERF})$, which is the result of linear regression using the survey data of Memmott and Dudek's study. The measured workzone capacity provided by Jiang's study $(1,466 \mathrm{vphpl})$ can be approximated using CERF $=0 \%$, and the one provided by Dudek and Richards' study (1,340 vphpl) can be approximated using CERF $=56 \%$. The default value is $\mathrm{CERF}=60 \%$, which can fit the average value of the survey data in Dudek and Richards' study.

The advantages of Jiang's study are that is more up-to-date, it is based on sampling from Indiana highways, and it provides more detailed information, such as the lane closure types (crossover or partial closure). Jiang analyzed 12 studies based on only four workzones, and all of which were the 2-to-1 type of lane closure. Because the lane 
closure types in Indiana are not limited to the 2-to-1 type, the measured workzone capacities for other types of lane closure are also needed for use in the HTIS. Memmott and Dudek's study, which is based on Dudek and Richards' study, included the 3-to-1, 2to-1, 5-to 2, 4-to-2, 3-to-2, and 4-to-3 lane closure types. In addition, their study allows planners to set the workzone capacities according to their needs by specifying different CERFs. The workzone capacity formula in Memmott and Dudek's study is adopted in the HTIS (Table 5.15).

Arrival rate

Arrival rates are expressed as hourly link volumes. When the "no information" link volumes are provided as the arrival rates, the resulting delay forms the upper bound of the expected delay at the workzone. When the "complete information" link volumes are provided as the arrival rates, the resulting delay forms the lower bound of the expected delay.

The concept of a queueing model is explained using the typical queueing diagram with two different arrival rates shown earlier (Figure 5.12). The first arrival rate $D_{l}$ is greater than the link service rate, so a queue builds. The second arrival rate $D_{2}$ is less than the link service rate, so the queue dissipates. This queueing model simulates a simple situation, in which a queue builds during the first time period and dissipates during the second period.

There are several possible combinations of arrival rates that result in different queueing scenarios. These scenarios are described below as they apply to the HTIS. Define $D_{1}, D_{2}$ and $A$ as the arrival rates during the AM-peak, PM-peak and off-peak periods, $T_{b 1}$ and $T_{e 1}$ as the beginning and the end of AM-peak periods, $T_{b 2}$ and $T_{e 2}$ as the 
beginning and the end of PM-peak periods, and $S$ as the service rate. The durations of AM-peak and PM-peak periods are equal to $\left(T_{e 1}-T_{b 1}\right)$ and $\left(T_{e 2}-T_{b 2}\right)$ respectively. The duration of AM-peak and PM-peak periods is set at three hours in the HTIS. However, the following queueing models derived in the HTIS can be applied to the other combinations of arrival rates and peak period durations, as long as the durations are expressed in integer hours. Figure A.8 shows how the user may make this specification. Scenario A: When the AM-peak period is not immediately followed by the PM-peak period and the first arrival rate is greater than the service rate

The queueing diagram for the situation when the AM-peak period is not immediately followed by the PM-peak period is shown in Figure 5.14.

Define $T_{q l}$ as the time when the queues that formed during an AM-peak period dissipate completely, and do so before the subsequent PM-peak period starts. $T_{q l}$ is calculated using the following equation.

cumulative number of vehicles in the queue that has just dissipated

$=$ cumulative number of vehicles served

$\Rightarrow D_{I}\left(T_{e l}-T_{b l}\right)+A\left(T_{q I}-T_{e l}\right)=S\left(T_{q I}-T_{b l}\right)$

$\Rightarrow T_{q 1}=\frac{\left(D_{1}-S\right)\left(T_{e 1}-T_{b 1}\right)}{(S-A)}+T_{e 1} \quad$ if $D_{l}>S$

The average queueing delay between period $t$ and $t+1$ for a queue forming in the AMpeak period is calculated as follows.

(1) $T_{b 1} \leq t<T_{e l}$

The total delay is the area of the shaded trapezoid area in Figure 5.14. 
Total delay $=\frac{1}{2}($ bottom length + top length $) \times$ height

$$
\begin{aligned}
& =\frac{1}{2}\left\{\left[\frac{D_{1}\left(t-T_{b 1}\right)}{S}-\left(t-T_{b 1}\right)\right]+\left[\frac{D_{1}\left(t+1-T_{b 1}\right)}{S}-\left(t+1-T_{b 1}\right)\right]\right\} \times D_{1} \\
& =\frac{1}{2} \frac{\left(D_{1}-S\right)\left(2 t+1-2 T_{b 1}\right)}{S} \times D_{1} \text { (veh-hr) }
\end{aligned}
$$

Average delay per vehicle $=\frac{\text { Total delay }}{\text { Number of vehicles arriving during the analysis period }}$

$$
\begin{aligned}
& =\frac{\frac{1}{2} \frac{\left(D_{1}-S\right)\left(2 t+1-2 T_{b 1}\right)}{S} \times D_{1}}{D_{1}} \times 60(\mathrm{~min}) \\
& =\frac{30 \cdot\left(D_{1}-S\right)\left(2 t+1-2 T_{b 1}\right)}{S}(\mathrm{~min})
\end{aligned}
$$

(2) $T_{e l} \leq t<T_{q l}$ and $t+1<T_{q l}$

The total delay is the area of the shaded triangle area in Figure 5.15.

Total delay $=\frac{1}{2}($ bottom length + top length $) \times$ height

$$
\begin{aligned}
& =\frac{1}{2}\left\{\left[\frac{D\left(T_{e 1}-T_{b 1}\right)+A\left(t-T_{e l}\right)}{S}-\left(t-T_{b 1}\right)\right]+\left[\frac{D\left(T_{e 1}-T_{b 1}\right)+A\left(t+1-T_{e l}\right)}{S}-\left(t+1-T_{b 1}\right)\right]\right\} \times A \\
& =\frac{1}{2} \frac{\left[2 D_{1}\left(T_{e 1}-T_{b 1}\right)+A\left(2 t+1-2 T_{e 1}\right)-S\left(2 t+1-2 T_{b 1}\right)\right]}{S} \times A(\text { veh-hr })
\end{aligned}
$$

Average delay per vehicle $=\frac{\text { Total delay }}{\text { Number of vehicles arriving during the analysis period }}$

$$
=\frac{\frac{1}{2} \frac{\left[2 D_{1}\left(T_{e 1}-T_{b 1}\right)+A\left(2 t+1-2 T_{e 1}\right)-S\left(2 t+1-2 T_{b 1}\right)\right]}{S} \times A}{A} \times 60(\mathrm{~min})
$$




$$
=\frac{30 \cdot\left[2 D_{1}\left(T_{e 1}-T_{b 1}\right)+A\left(2 t+1-2 T_{e 1}\right)-S\left(2 t+1-2 T_{b 1}\right)\right]}{S}(\min )
$$

(3) $T_{e l} \leq t<T_{q I}$ and $t+1 \geq T_{q I}$

Because the queue dissipates completely at $T_{q 1}$, there is no queueing delay between $T_{q l}$ and $(t+1)$. The total delay is the area of the shaded triangle area in Figure 5.16.

Total delay $=\frac{1}{2}($ bottom length $\times$ height $)$

$$
\begin{aligned}
& =\frac{1}{2}\left[\frac{D_{1}\left(T_{e 1}-T_{b 1}\right)+A\left(t-T_{e 1}\right)}{S}-\left(t-T_{b 1}\right)\right] \times \frac{D_{1}\left(T_{e 1}-T_{b 1}\right)+A\left(T_{q 1}-T_{e 1}\right)}{S} \\
& =\frac{1}{2} \frac{\left[D_{1}\left(T_{e 1}-T_{b 1}\right)+A\left(t-T_{e 1}\right)-S\left(t-T_{b 1}\right)\right]^{2}}{S(S-A)} \times A(\text { veh-hr })
\end{aligned}
$$

Average delay per vehicle $=\frac{\text { Total delay }}{\text { Number of vehicles arriving during the analysis period }}$

$$
\begin{aligned}
& =\frac{\frac{1}{2} \frac{\left[D_{1}\left(T_{e 1}-T_{b 1}\right)+A\left(t-T_{e 1}\right)-S\left(t-T_{b 1}\right)\right]^{2}}{S(S-A)} \times A}{A} \times 60(\mathrm{~min}) \\
& =\frac{30 \cdot\left[D_{1}\left(T_{e 1}-T_{b 1}\right)+A\left(t-T_{e 1}\right)-S\left(t-T_{b 1}\right)\right]^{2}}{S(S-A)}(\mathrm{min})
\end{aligned}
$$

The average queueing delay between period $t$ and $t+1$ for a queue forming in the PMpeak period is calculated in a similar way to that during the AM-peak period illustrated above. Therefore, only the final results are shown below.

Define $T_{q 2}$ as the time when the queues formed during a PM-peak period dissipate completely.

$$
T_{q 2}=\frac{\left(D_{2}-S\right)\left(T_{e 2}-T_{b 2}\right)}{(S-A)}+T_{e 2} \quad \text { if } D_{2}>S
$$


(1) $T_{b 2} \leq t<T_{e 2}$

Average delay per vehicle $=\frac{30 \cdot\left(D_{2}-S\right)\left(2 t+1-2 T_{b 2}\right)}{S}(\min )$

(2) $T_{e 2} \leq t<T_{q 2}$ and $t+1<T_{q 2}$

Average delay per vehicle $=\frac{30 \cdot\left[2 D_{2}\left(T_{e 2}-T_{b 2}\right)+A\left(2 t+1-2 T_{e 2}\right)-S\left(2 t+1-2 T_{b 2}\right)\right]}{S}(\min )$

(3) $T_{e 2} \leq t<T_{q 2}$ and $t+1 \geq T_{q 2}$

Average delay per vehicle $=\frac{30 \cdot\left[D_{2}\left(T_{e 2}-T_{b 2}\right)+A\left(t-T_{e 2}\right)-S\left(t-T_{b 2}\right)\right]^{2}}{S(S-A)}(\mathrm{min})$

Scenario B: When the AM-peak period is immediately followed by the PM-peak period and the first arrival rate is greater than the service rate

If the PM-peak period begins immediately after the AM-peak period and a queue forms in the AM-peak period, the queue may not be dissipated completely by the beginning of PM-peak period. Figure 5.17 shows two types of queueing diagrams for situations when the PM-peak period begins immediately after the AM-peak period. By applying the same calculation process as in the previous case, the queueing delays are obtained. The following paragraphs list the final results of the calculation processes.

Define $T_{q 3}$ as the time when the queue dissipates completely after the PM-peak period, and $T_{q^{4}}$ as the time when the queue dissipates completely after the AM-peak period but before the PM-peak period ends.

$$
\begin{aligned}
& T_{q 3}=\frac{D_{1}\left(T_{e 1}-T_{b 1}\right)+D_{2}\left(T_{e 2}-T_{e 1}\right)-A T_{e 2}+S T_{b 1}}{S-A} \\
& T_{q 4}=\frac{\left(D_{1}-S\right)\left(T_{e 1}-T_{b 1}\right)}{\left(S-D_{2}\right)}+T_{e 1}
\end{aligned}
$$


a. When the queue does not dissipate completely until after the PM-peak period ends

(1) $T_{b 1} \leq t<T_{e l}$

Average delay per vehicle $=\frac{30 \cdot\left(D_{1}-S\right)\left(2 t+1-2 T_{b 1}\right)}{S}(\mathrm{~min})$

(2) $T_{e l}\left(=T_{b 2}\right) \leq t<T_{e 2}$

Average delay per vehicle

$=\frac{30 \cdot\left[2 D_{1}\left(T_{e 1}-T_{b 1}\right)+D_{2}\left(2 t+1-2 T_{e l}\right)-S\left(2 t+1-2 T_{b 1}\right)\right]}{S}(\min )$

(3) $T_{e 2} \leq t<T_{q 3}$ and $t+1<T_{q 3}$

Average delay per vehicle

$=\frac{30 \cdot\left[2 D_{1}\left(T_{e 1}-T_{b 1}\right)+2 D_{2}\left(T_{e 2}-T_{e 1}\right)+A\left(2 t+1-2 T_{e 2}\right)-S\left(2 t+1-2 T_{b 1}\right)\right]}{S}(\min )$

(4) $T_{e 2} \leq t<T_{q 3}$ and $t+1 \geq T_{q 3}$

Average delay per vehicle

$=\frac{30 \cdot\left[D_{1}\left(T_{e 1}-T_{b 1}\right)+D_{2}\left(T_{e 2}-T_{e 1}\right)+A\left(t-T_{e 2}\right)-S\left(t-T_{b 1}\right)\right]^{2}}{S(S-A)}(\mathrm{min})$

b. When the queue dissipates completely after the AM-peak period but during the PMpeak period

(1) $T_{b 1} \leq t<T_{e l}$

Average delay per vehicle $=\frac{30 \cdot\left(D_{1}-S\right)\left(2 t+1-2 T_{b 1}\right)}{S}(\mathrm{~min})$

(2) $T_{e 1} \leq t<T_{q 4}$ and $t+1<T_{q 4}$

Average delay per vehicle 


$$
=\frac{30 \cdot\left[2 D_{1}\left(T_{e 1}-T_{b 1}\right)+D_{2}\left(2 t+1-2 T_{e 1}\right)-S\left(2 t+1-2 T_{b 1}\right)\right]}{S}(\min ),
$$

(3) $T_{e l} \leq t<T_{q^{4}}$ and $t+1 \geq T_{q 4}$

$$
\text { Average delay per vehicle }=\frac{30 \cdot\left[D_{1}\left(T_{e 1}-T_{b 1}\right)+D_{2}\left(t-T_{e 1}\right)-S\left(t-T_{b 1}\right)\right]^{2}}{S\left(S-D_{2}\right)}(\mathrm{min})
$$

The above formulas were verified by comparing their results with experimental data from traffic surveys. As explained at the beginning of Section 5.5, two basic inputs hourly volumes and capacities - are required for queueing analysis. The workzone capacity represents the service rate of the link that is under construction. The workzone capacities are based on Memmott and Dudek's study (1982) (Table 5.15). The default value of $60 \%$ for $\mathrm{CERF}$ is used. Hourly volumes represent the arrival rates of the link. The demands on each link vary, not only for different days, but also for different times of day. Therefore, field measurements are required to obtain hourly volumes. Because each hourly volume requires an hour of survey data, using the data from existing ATR stations near the workzone to be analyzed was considered as an alternative. If an ATR station is located immediately upstream of the workzone link, the data recorded at the ATR station can be used as the demand on the workzone link.

One workzone with an ATR station near it was on I-65 between SR43 and SR18. ATR station 5450 is located just south of SR43. Figure 5.18 and 5.19 show where traffic surveys were conducted. There were four lanes on I-65 between SR 43 and SR 18 before construction. The construction was done in two phases. During the first phase, the two southbound lanes were closed. Southbound traffic was diverted to one lane on the northbound side of the median. The southbound ramps at the SR18 interchange were 
closed, too. An alternate route via SR43 northbound to SR18 and westbound back to I-65 for northbound traffic was available to bypass the workzone.

During phase two, the two northbound lanes were closed. Northbound traffic was diverted to one lane on the southbound side of the median. The northbound ramps at SR18 interchange were closed. An alternate route via SR43 northbound to SR18, eastbound to SR231 and northbound back to I-65 for northbound traffic was available to bypass the workzone.

In order for queues to form queueing situations, hourly volumes must be greater than the workzone capacity. The workzone capacity for the 2-to-1 type of lane closure in each direction, based on Memmott and Dudek's study (1982) using the default value of 60\% for CERF is 1,332 vphpl. Judging from the historical hourly volumes of station 5450 , the northbound traffic on Sunday afternoon has a high probability of exceeding workzone capacity. Therefore, two surveys were conducted - both on the northbound traffic on Sunday afternoon.

\section{A. First traffic survey}

The first traffic survey was conducted between 4:00PM and 6:00PM on 9 May 1999 during construction phase one. (See Figure 5.18.) The survey items included the average travel time through the I- 65 workzone between SR 43 and SR18, the average travel time via the alternate route, and northbound on-ramp and off-ramp counts at the SR43 interchange.

(1) Average measured travel time through the I-65 workzone between SR43 and SR18 The average measured travel time through the I-65 workzone between SR 43 and SR18 consists two parts -SR43 to CR600N and CR600N to SR18. The average travel 
time between SR43 and CR600N was based on the average speed observed at CR600N. The average speed measured at CR600N is $14.59 \mathrm{mph}$ and the distance between SR43 and CR600N of 1.29 mile. Hence, the travel time is $(1.29 / 14.59) \times 60=5.3$ minutes.

The average travel time between CR600N and SR18 was obtained by tracing the travel time of sampled vehicles from the videotapes of two synchronized video cameras at $\mathrm{CR} 600 \mathrm{~N}$ and at SR18. A total number of 109 vehicles were sampled. The average travel time for the sampled vehicles is 13.8 minutes.

The average observed travel time through the I- 65 workzone from SR43 to SR18 is the sum of the two values above (the average travel time between SR43 and CR600N and the average travel time between CR600N and SR18), which is 19.1 minutes. This value was compared with the estimated travel time from the sum of queueing delay and freeflow travel time to verify the accuracy of the delay calculation.

(2) Average travel time via the alternate route

The travel time via the alternate route was obtained from the average travel time of repeated runs by a probe vehicle. The resulting average travel time through the alternate route was 16.0 minutes. This value was compared with the average measured travel time through the I-65 workzone to see if drivers can save time by traveling over the alternate route.

(3) Northbound on-ramp and off-ramp counts at the SR43 interchange

The northbound on-ramp and off-ramp counts at the SR43 interchange are also required because ATR station 5450 is located south of SR43 and some vehicles may get on and off at the SR 43 interchange. The northbound demands on I-65 between SR43 and SR18 were determined from the northbound hourly volumes recorded at station 5450 
minus the northbound off-ramp count, plus the northbound on-ramp count at the SR43 interchange. Because of limited time and resources, the northbound on- and off-ramp counts were observed during the time period when a queue is most likely to build (5:00PM and 6:00PM). The northbound on- and off-ramp counts during other periods are of less concern to the HTIS, because queues are less likely to build then. The on- and off-ramp counts during these "other periods" were estimated based on the ratio of the onand off-ramp traffic to the hourly volume between 5:00PM and 6:00PM.

The estimated travel time through the I-65 workzone from SR43 to SR 18 is the sum of the free-flow travel time and the queueing delay caused by the I-65 workzone.

(1) Free-flow travel time

The free-flow travel time is obtained by dividing the free-flow speed on I-65 (70 $\mathrm{mph}$ ) by the distance on I- 65 between SR43 and SR18 ( 8 miles). The free-flow travel time on I- 65 from SR 43 to SR 18 is 6.86 minutes.

(2) Queueing delay caused by the I-65 workzone

A queue forms when the arrival rate is greater than the service rate. The arrival rates at the I-65 workzone are the hourly volumes obtained from the data at ATR station 5450, adjusted by the northbound ramp counts at the SR 43 interchange. The arrival rates between 3:00PM and 8:00PM $(1357,1469,1564,1579$ and1373 vph) are greater than the service rate (1332 vph). Although the queue was observed to grow, dissipate, and grow again several times during this 5-hour period, the 5 hourly arrival rates were used in a queueing diagram analysis that began at 3:00PM. This simplification provided results that were conservative, but close to observed delay values. The queueing diagram for this survey is presented in Figure 5.20. 
The total queueing delay and average delay per vehicle between 5:00PM and 6:00PM are calculated as follows:

$$
\begin{aligned}
\text { Total delay } & =\frac{1}{2}(\text { bottom length }+ \text { top length }) \times \text { height } \\
& =\frac{1}{2}\left\{\left[\frac{1357+1436}{1332}-(17-15)\right]+\left[\frac{1357+1436+1564}{1332}-(18-15)\right]\right\} \times 1564 \\
& \approx 287.67(\text { veh-hr })
\end{aligned}
$$

Average delay per vehicle $=\frac{\text { Total delay }}{\text { Number of vehicles arriving during the analysis period }}$

$$
\begin{aligned}
& =\frac{287.67}{1564} \times 60 \text { minutes } \\
& =11.04 \text { minutes }
\end{aligned}
$$

The estimated travel time is the sum of the free-flow travel time and the average queueing delay per vehicle, which is 17.9 minutes. The comparison of measured and estimated travel time is presented in Table 5.16. A discussion is given after the descriptions of the second traffic survey.

B. Second traffic survey

The second traffic survey was conducted between 4:00PM and 6:00PM on 20 June 1999 during construction phase two when two northbound lanes were closed and the southbound traffic was diverted to one lane on the northbound side of the median. A map is presented in Figure 5.19. Because the northbound ramps were closed, the alternate route for northbound traffic in the first stage was modified to be northbound SR43 to SR18, westbound SR18 to US231, and northbound SR231 back to I-65. The survey data items include: (1) average travel time through workzone between SR43 and US231; (2) average travel time via the alternative route; and (3) northbound ramp counts at SR43. The methods applied to obtain the second and third items are the same as in the first traffic survey. The first item was obtained from the average travel time from repeated 
runs of a probe vehicle, instead of using two video cameras to track vehicles between two ends, because video taping equipment was not available at the time of the second traffic survey.

The calculation process for the second survey is similar to the first one. The results of the first two survey items in the second survey are 20.35 minutes for the average travel time through workzone between SR43 and US231 and 22.43 minutes for the average travel time via the alternative route. The average queueing delay per vehicle caused by the I-65 workzone is 9.39 minutes and the free-flow travel time on I-65 from SR43 to US231 is 10.8 minutes. The estimated travel time through workzone between SR43 and US231 is the free-flow travel time plus the queueing delay, which is 20.19 minutes.

The comparison of the measured travel time and estimated travel time is presented in Table 5.16. The percentage difference is defined as the difference between the measured travel time and the estimated travel time, divided by the measured travel time. The percentage of difference for the first traffic survey is $6.3 \%$, and $0.8 \%$ for the second traffic survey. The estimated travel times from both traffic surveys are close to the measured travel time. Although two traffic surveys may not be sufficient to draw a strong conclusion as to the accuracy of the estimated queueing delays, these survey results can justify the use of the queueing analysis in the HTIS.

\subsubsection{Delay Refinement}

Delay refinement plays an important role in the HTIS. The function of delay refinement is to estimate delays during the transition time when the existence of workzones is known only to a small portion of travelers. As construction continues, more 
travelers gain knowledge of the existence of workzones through various kinds of media. Some of the informed travelers may change their choices of routes and departure time in order to reduce delays. The aggregate effect on travelers' behavior may result in changes in expected delays before the system reaches an equilibrium status. By understanding how traffic demands change as awareness about the workzone spreads, ...

- Modelers may be able to determine how faster and more extensive dissemination of the workzone information can reduce the transition time to the new equilibrium flow pattern.

- Project schedulers may consider the "no information" traffic conditions, the transition time, and the new equilibrium in determining a project scheduling strategy that avoids inducing excessive delays.

- Analysts can decide whether a more extensive HTIS is justified to speed up the transition to a new equilibrium.

There are two possible traffic conditions during the transition time: (1) the demand on the workzone link remains higher than the workzone capacity; (2) the demand on the workzone link reduces to less than the workzone capacity. In the first traffic condition, the delay on the workzone link will continue even after a new equilibrium flow pattern is established. The delay in this traffic condition falls in between the upper and lower bounds estimated in Section 5.5.1. In the second traffic condition, delay on the workzone link decreases to zero before the traffic reaches the equilibrium flow pattern. However, the volumes on the workzone link in the above two traffic conditions show a similar decreasing trend over the age of construction. Therefore, by establishing a relationship between the workzone volumes and the age of construction, the volumes on the workzone 
link during the transition time can be estimated. The delays on the workzone link during the transition time can then be estimated based on the estimated link volumes.

Several approaches were attempted in order to establish the relationship between workzone volumes and the age of construction. The first approach would be to use the historical data for ATR stations located upstream of a workzone link. The flow at the upstream ATR station can be used to represent the demand on the workzone link when the following two criteria are met: (1) the flow is not affected by the queue downstream; and (2) no ramps exist between the ATR station and the workzone. This approach could not be used because no ATR stations and workzones could be found that met both criteria mentioned above. Some cases failed because the ATR stations were too close to the workzones to be considered unaffected by the queue downstream. Some cases failed because ramps existed between the ATR stations and the workzones. In the few cases where the above two criteria were met, incomplete ATR data prevented the development of any relationship between the workzone volumes and the age of construction.

The second approach to derive a relationship between workzone volumes and age of construction is to conduct traffic surveys to observe the flow upstream of a workzone as it continues. A two-week traffic survey between 4:00PM and 5:00PM from 26 April 2000 to 9 May 2000 was conducted on the northbound approach to a workzone on I-65 between SR26 and SR43. (See Figure 5.21.) The road project just south of ATR Station 5450 started on 24 April 2000 and closed the two southbound lanes. ATR Station 5450 is located downstream of the workzone. Because workzone queues would reduce traffic flow rates, data from a downstream ATR cannot be used to measure the demand on the workzone link. The demand on the workzone link was estimated using the northbound 
flow observed upstream of SR26, minus the observed off-ramp flow at the SR26 interchange, plus the observed on-ramp flow.

The plot of the traffic demands on the workzone link from the survey versus the age of construction is shown in Figure 5.22. The demands from the April-May 2000 survey were compared with adjusted historical ATR volumes taken when no construction was present nearby. The adjustments were made with annual growth factors defined in Section 5.2. The most recent historical volumes at ATR Station 5450 without construction projects in the months of April and May were taken in 1996. The plot of the volumes at ATR Station 5450 in Figure 5.22 begins on the last Wednesday in April 1996 and 2000. The linear regression line for the demands from the April-May 2000 survey shows a downward trend; and the one for the growth-adjusted 1996 volumes shows a slightly increasing trend. The downward trend for the demands from the survey is more obvious when the demands on weekends are excluded (Figure 5.23). Although the data from a single survey cannot be used to determine the mathematical function of the downward trend, they display the influence of the workzone on driver choice during the transition period. Therefore, the hypothesis that volumes on the workzone link decrease as construction continues is applied to the "no information" and the "complete information" volumes to estimate the volumes on weekdays during the transition period. These estimated volumes are then used to estimate the delays on weekdays during the transition period.

The termination of the downward trend for the demands from the survey is estimated by comparing the data from the first week of the survey with the second week (Figure 5.24). A negative value of the difference between the demands in the second week and 
the demands in the first week indicates that demand is decreasing; and a positive value represents the demand is increasing. In Figure 5.24, the value becomes positive on Friday, which is the tenth weekday of construction. Therefore, it is assumed (for now) that the system reaches equilibrium at the tenth weekday. Of course, as further data become available, this value can be revised.

The demands from the survey on weekends do not show a great difference from the volumes in 1996 (Figure 5.22). A possible explanation is that the weekend trips are longer and involve non-local travelers. It is assumed that the weekend patterns are not influenced by construction. Long-distance tripmakers may be infrequent users of the road under construction and may not find out about the wokrzone until it has been in place several weeks. Even if aware of the workzone, long-distance drivers may prefer to wait in the queue rather than trying to find a detour route in unfamiliar territory. Consequently, "no information" volumes are applied to the delay estimation for weekends during the transition period.

The processes for estimating the delays on the workzone link on weekdays during the transition time applied in the HTIS are divided into two parts: estimation of the volumes on the workzone link and estimation of delay on the workzone link during the transition time.

A. Estimation of the volumes on the workzone link

The volume on the workzone link for any given day and hour(s) during the transition time is estimated by applying a hypothesized relationship with the age of construction, constrained by the upper bound and lower bound volumes estimated from the "no information" and the "complete information" analyses. 
The hypothesized relationship is a logistic function. A logistic function has been widely used for estimating stochastic situations such as modal split in transportation planning. A typical 'decreasing' logistic function decreases exponentially at the beginning and flattens toward an asymptote (Figure 5.21), which simulates the changes of demand on the workzone link as the construction continues. Most frequent travelers may obtain information about the new or upcoming workzone either from various news media or by traveling through it at the beginning of the construction project. These frequent travelers may make informed decisions to divert to alternate routes or to start their trips at a different time. The volume on the workzone link can be expected to decrease quickly from the "no information" value at the beginning of the construction project. As the construction continues, more travelers become aware of the workzone and make informed decisions on route choices and departure times. The rate at which the volume decreases becomes smaller. The traffic through the workzone eventually reaches an equilibrium. Because there are always certain number of uninformed travelers who are not aware of the workzone, the "complete information" condition cannot be reached. The percentage of these uninformed travelers is represented as the gap between the "complete information" value and the asymptote in Figure 5.25.

Define $y$ as the volume on the workzone link, $x$ as the age of the workzone, $U$ as the "no information" link volume, $L$ as the "complete information" link volume, $E$ as the volume at the steady state, while the percentage of uninformed travelers on the workzone link is $p_{u}$. The difference between the volume at the steady state and the "complete information" link volume equals the number of uninformed travelers on the workzone link. That is, ... 


$$
\begin{aligned}
& (E-L)=p_{u} \times(U-L) \\
& \Rightarrow E=p_{u}(U-L)+L
\end{aligned}
$$

The difference between the 'no information' link volume and the volume at steady state $(U-E)$ is then $\left(1-p_{u}\right)(U-L)$.

The relationship between the volume on the workzone link and the age of the construction zone using a three-parameter logistic function is as follows (Thatcher, Kannisto and Vaupel, 1998).

$$
\begin{aligned}
y & =\left(1-\frac{1}{1+c e^{-k x}}\right) \times(U-E) \\
& =\left(1-\frac{1}{1+c e^{-k x}}\right) \times\left(\left(1-p_{u}\right)(U-L)\right)
\end{aligned}
$$

where $c$ and $k$ are positive coefficients, and $0<p_{u}<1$

The $k$ in the above formula determines the decreasing rate of the workzone volumes approaching the asymptote; and the $c$ affects the intercept on axis $\mathrm{Y}$.

The intercept on the Y axis in the HTIS is the difference between the 'no information' link volume and the volume at steady state.

$$
\begin{aligned}
& y=\left(1-\frac{1}{1+c e^{-k \cdot 0}}\right) \times\left(\left(1-p_{u}\right)(U-L)\right)=\left(\left(1-p_{u}\right)(U-L)\right) \\
& \Rightarrow c>>0
\end{aligned}
$$

The $c$ is set as 100 under the assumption that the error percentage for the link volume is less than $5 \%$. Therefore, the logistic function can be expressed as follows.

$$
y=\left(1-\frac{1}{1+100 \cdot e^{-k x}}\right) \times\left(\left(1-p_{u}\right)(U-L)\right)
$$


Define that the system reaches a steady state when the total percentage of changes on the link volume to the difference between the 'no information' link volume and the volume at steady state at time $T$ is equal to $p_{e}(S S$ in Figure 5.25). That is, there are (1$\left.p_{e}\right)\left(\left(1-p_{u}\right)(U-L)\right)$ frequent travelers to be diverted before the system reaches the "true" equilibrium.

$$
\begin{aligned}
& \left(1-p_{e}\right)\left(\left(1-p_{u}\right)(U-L)\right)=\left(1-\frac{1}{1+100 \cdot e^{-k T}}\right) \times\left(\left(1-p_{u}\right)(U-L)\right) \\
& \Rightarrow 1-p_{e}=1-\frac{1}{1+100 \cdot e^{-k T}} \\
& \Rightarrow k=\frac{1}{T} \ln \frac{100 \cdot p_{e}}{1-p_{e}}
\end{aligned}
$$

The $S S$ point in Figure 5.25 defined by the values of $T$ and $p_{e}$ determines the parameter $k$, which is related to the decreasing rate of the workzone volumes approaching the asymptote. For example, if the workzone flow rate reaches a steady state that is 95 percent of the way from the $\mathrm{U}$ level to the $\mathrm{L}$ value after 10 days, then $T=10$ and $p_{e}=$ 0.95 .

$$
k=\frac{1}{10} \ln \frac{100 \times .95}{1-.95}=0.755
$$

The relationship between the volume on the workzone link and the age of construction can be defined as follows.

$$
y=\left(1-\frac{1}{1+100 \cdot e^{-0.755 x}}\right) \times\left(\left(1-p_{u}\right)(U-L)\right)
$$

By defining the percentage of uninformed travelers, the link volumes during the transition time can be estimated as follows. 
Link volume $=y+E$

$$
\begin{aligned}
& =y+p_{u}(U-L)+L \\
& =\left(1-\frac{1}{1+100 \cdot e^{-0.755 x}}\right) \times\left(\left(1-p_{u}\right)(U-L)\right)+\left(p_{u}(U-L)+L\right)
\end{aligned}
$$

For example, if the upper bound and lower bound volumes of a link are 3,000 vph and $1,000 \mathrm{vph}$, respectively, the link volume for the cases when the percentage of uninformed travelers $p_{u}$ is $10 \%$ and $70 \%$ can be estimated using the following formula.

$$
\begin{aligned}
& \text { Link volume }_{p u=10 \%}=\left(1-\frac{1}{1+100 \cdot e^{-0.755 x}}\right) \times((1-0.1) U-L)+(0.1 \cdot(U-L)+L) \\
& \text { Link volume }{ }_{p u=70 \%}=\left(1-\frac{1}{1+100 \cdot e^{-0.755 x}}\right) \times((1-0.7) U-L)+(0.7 \cdot(U-L)+L)
\end{aligned}
$$

The logistic curves for the above two cases are shown in Figure 5.26. The higher percentage of uninformed travelers, the larger gap exists between the steady state and the lower bound volumes.

B. Estimation of delay on the workzone link during the transition time

The delay on the workzone link during the transition time is estimated by applying queueing theory to the estimated link volume. The application of queueing theory to calculate the queueing delays caused by construction is explained in Section 5.5.1. By defining the arrival rate as the volume for a specified day and hour(s) during the transition time and the service rate as the workzone capacity, the delay on the workzone link during the transition time can be obtained. 
Table $5.1 r_{s}$ of Spearman Rank Correlation Test on ATR Station 2400 (November $3 \sim$ November 9, 1997)

\begin{tabular}{|c|c|c|c|c|c|c|c|}
\hline Date & $\begin{array}{c}11 / 3 \\
\text { Mon }\end{array}$ & $\begin{array}{c}11 / 4 \\
\text { Tue }\end{array}$ & $\begin{array}{c}11 / 5 \\
\text { Wed }\end{array}$ & $\begin{array}{c}11 / 6 \\
\text { Thu }\end{array}$ & $\begin{array}{c}11 / 7 \\
\text { Fri }\end{array}$ & $\begin{array}{c}11 / 8 \\
\text { Sat }\end{array}$ & $\begin{array}{c}11 / 9 \\
\text { Sun }\end{array}$ \\
\hline $11 / 3$ Mon & 1.00 & 0.97 & 0.98 & 0.98 & 0.93 & 0.69 & 0.53 \\
\hline $11 / 4$ Tue & 0.97 & 1.00 & 0.98 & 0.98 & 0.90 & 0.71 & 0.51 \\
\hline $11 / 5$ Wed & 0.98 & 0.98 & 1.00 & 0.99 & 0.92 & 0.71 & 0.47 \\
\hline $11 / 6$ Thu & 0.98 & 0.98 & 0.99 & 1.00 & 0.95 & 0.74 & 0.52 \\
\hline $11 / 7$ Fri & 0.93 & 0.90 & 0.92 & 0.95 & 1.00 & 0.77 & 0.62 \\
\hline $11 / 8$ Sat & 0.69 & 0.71 & 0.71 & 0.74 & 0.77 & 1.00 & 0.80 \\
\hline $11 / 9$ Sun & 0.53 & 0.51 & 0.47 & 0.52 & 0.62 & 0.80 & 1.00 \\
\hline
\end{tabular}

(Note) The shaded area indicates that the traffic patterns for weekdays are similar. 
Table 5.248 periods ahead forecasts at base period 160 and 24 periods ahead updated forecasts at base period 184 (November 1997)

\begin{tabular}{|c|c|c|c|c|c|}
\hline Time & $\begin{array}{c}95 \% \text { lower } \\
\text { confidence limit }\end{array}$ & $\begin{array}{c}\text { Forecast value } \\
\text { (base } 160 \text { ) }\end{array}$ & $\begin{array}{c}95 \% \text { upper } \\
\text { confidence limit }\end{array}$ & $\begin{array}{l}\text { Actual value } \\
\text { if known }\end{array}$ & $\begin{array}{c}\text { Forecast value } \\
\text { (base 184) }\end{array}$ \\
\hline 161 & 898.55 & 971.66 & 1044.78 & 1058 & \\
\hline 162 & 920.71 & 1005.77 & 1090.82 & 1051 & \\
\hline 163 & 724.90 & 813.79 & 902.68 & 879 & \\
\hline 164 & 512.00 & 602.20 & 692.41 & 617 & \\
\hline 165 & 390.19 & 480.86 & 571.53 & 522 & \\
\hline 166 & 358.04 & 448.87 & 539.70 & 422 & \\
\hline 167 & 306.66 & 397.54 & 488.43 & 378 & \\
\hline 168 & 269.45 & 360.35 & 451.26 & 337 & \\
\hline 169 & 203.35 & 294.27 & 385.18 & 305 & \\
\hline 170 & 147.91 & 238.83 & 329.75 & 257 & \\
\hline 171 & 122.57 & 213.49 & 304.41 & 227 & \\
\hline 172 & 122.00 & 212.92 & 303.84 & 256 & \\
\hline 173 & 148.59 & 239.50 & 330.42 & 269 & \\
\hline 174 & 320.94 & 411.86 & 502.77 & 435 & \\
\hline 175 & 837.93 & 928.85 & 1019.77 & 996 & \\
\hline 176 & 1313.31 & 1404.22 & 1495.14 & 1463 & \\
\hline 177 & 944.19 & 1035.11 & 1126.02 & 942 & \\
\hline 178 & 806.00 & 896.92 & 987.84 & 941 & \\
\hline 179 & 709.52 & 800.44 & 891.35 & 861 & \\
\hline 180 & 676.23 & 767.15 & 858.07 & 784 & \\
\hline 181 & 660.21 & 751.13 & 842.05 & 753 & \\
\hline 182 & 714.59 & 805.51 & 896.42 & 844 & \\
\hline 183 & 781.21 & 872.12 & 963.04 & 838 & \\
\hline 184 & 866.40 & 957.31 & 1048.23 & 1148 & \\
\hline 185 & 904.07 & 996.04 & 1088.00 & 1060 & 1125.69 \\
\hline 186 & 918.77 & 1011.26 & 1103.76 & 1082 & 1065.78 \\
\hline 187 & 724.55 & 817.23 & 909.91 & 779 & 858.38 \\
\hline 188 & 520.69 & 613.44 & 706.18 & 526 & 623.87 \\
\hline 189 & 392.89 & 485.66 & 578.43 & 471 & 503.91 \\
\hline 190 & 351.95 & 444.73 & 537.51 & 372 & 437.86 \\
\hline 191 & 312.28 & 405.06 & 497.84 & 457 & 413.03 \\
\hline 192 & 277.68 & 370.46 & 463.24 & 365 & 373.86 \\
\hline 193 & 197.27 & 290.05 & 382.83 & 211 & 299.63 \\
\hline 194 & 140.64 & 233.42 & 326.21 & 165 & 235.25 \\
\hline 195 & 118.45 & 211.24 & 304.02 & 125 & 209.91 \\
\hline 196 & 120.76 & 213.54 & 306.32 & 127 & 218.70 \\
\hline 197 & 150.43 & 243.21 & 336.00 & 166 & 238.33 \\
\hline 198 & 323.91 & 416.70 & 509.48 & 374 & 413.89 \\
\hline 199 & 831.01 & 923.79 & 1016.58 & 836 & 930.82 \\
\hline 200 & 1306.10 & 1398.88 & 1491.66 & 1360 & 1393.39 \\
\hline 201 & 959.24 & 1052.02 & 1144.81 & 1024 & 1019.89 \\
\hline 202 & 810.45 & 903.23 & 996.01 & 784 & 934.65 \\
\hline 203 & 710.85 & 803.63 & 896.42 & 769 & 804.16 \\
\hline 204 & 670.33 & 763.11 & 855.89 & 675 & 751.29 \\
\hline 205 & 659.07 & 751.85 & 844.64 & 689 & 748.04 \\
\hline 206 & 721.24 & 814.02 & 906.80 & 715 & 820.83 \\
\hline 207 & 771.47 & 864.25 & 957.03 & 793 & 848.26 \\
\hline 208 & 858.42 & 951.21 & 1043.99 & 850 & 995.70 \\
\hline
\end{tabular}




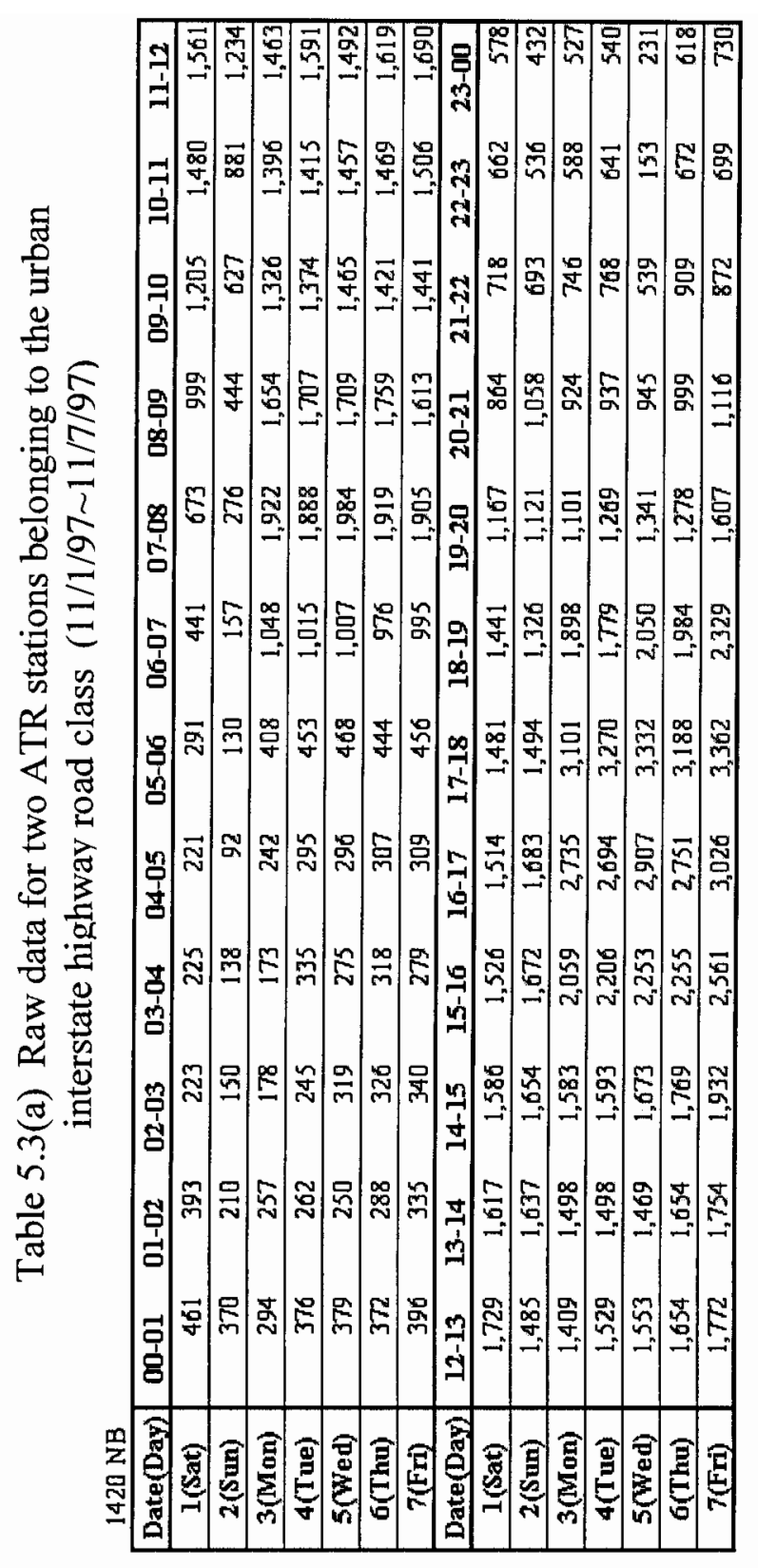

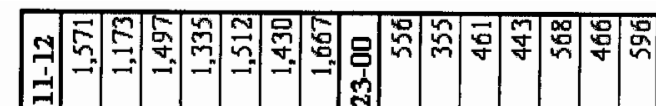

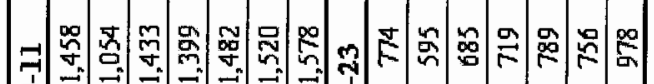
$\Rightarrow-1-1-2$

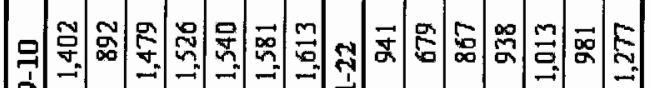
음

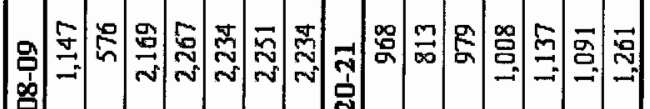
80 (1) m m

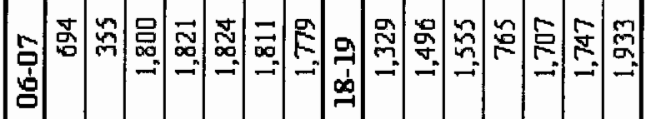

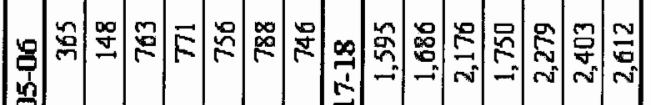
.

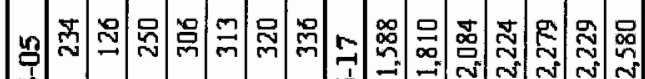
I

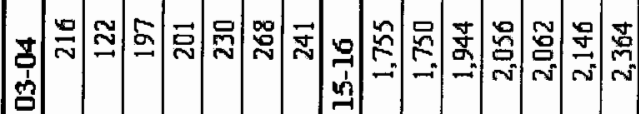

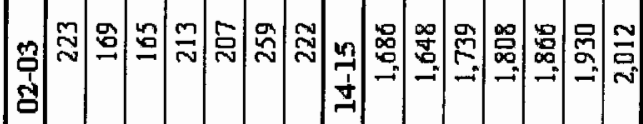

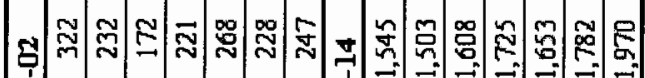
용

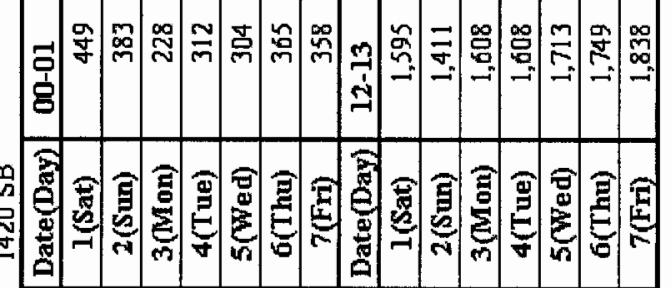


Table 5.3(b) Raw data for two ATR stations belonging to the urban interstate highway road class (11/1/97 11/7/97)

$2400 \mathrm{NB}$

\begin{tabular}{|c|c|c|c|c|c|c|c|c|c|c|c|c|}
\hline Date(Day) & 00-01 & 01-02 & $02-03$ & 03-04 & 04-05 & 05-06 & $06-07$ & $07-08$ & 08-09 & 09-10 & $10-11$ & $11-12$ \\
\hline l(Sat) & 253 & 185 & 177 & 168 & 158 & 213 & 306 & 421 & 551 & 629 & 736 & 827 \\
\hline 2 (Sun) & 249 & 170 & 133 & 140 & 109 & 100 & 154 & 244 & 359 & 501 & 713 & 946 \\
\hline 3(Mon) & 328 & 225 & 178 & 200 & 197 & 304 & 565 & 694 & 690 & 669 & 709 & 827 \\
\hline 4(Tue) & 258 & 211 & 191 & 213 & 215 & 314 & 527 & 695 & 681 & 670 & 688 & 733 \\
\hline S(Wed) & 278 & 211 & 207 & 198 & 231 & 306 & 527 & 658 & 682 & 701 & 684 & 780 \\
\hline 6(Thu) & 273 & 256 & 213 & 264 & 242 & 285 & 592 & 724 & 743 & 742 & 723 & 774 \\
\hline 7(Fri) & 316 & 250 & 237 & 234 & 270 & 360 & 580 & 703 & 710 & 795 & 916 & 954 \\
\hline Date(Day) & $12-13$ & $13-14$ & $14-15$ & $15-16$ & $16-17$ & $17-18$ & $18-19$ & $19-20$ & $20-21$ & $21-22$ & $22-23$ & 23-00 \\
\hline 1 (Sat) & 814 & 825 & 979 & 1,042 & 1,009 & 961 & 846 & 649 & 565 & 592 & 500 & 405 \\
\hline 2(Sun) & 1,093 & 1,173 & 1,225 & 1,356 & 1,647 & 1,653 & 1,367 & 1,057 & 801 & 620 & 481 & 381 \\
\hline 3(Mon) & 818 & 720 & 853 & 1,062 & 1,309 & 1,391 & 880 & 610 & 611 & 470 & 363 & 283 \\
\hline 4(Tue) & 742 & 739 & 791 & 1,084 & 1,339 & 1,373 & 939 & 697 & 586 & 483 & 406 & 312 \\
\hline 5(Wed) & 817 & 750 & 846 & 1,081 & 1,327 & 1,434 & 929 & 734 & 635 & 526 & 437 & 372 \\
\hline 6(Thu) & 808 & 792 & 945 & 1,170 & 1,414 & 1,481 & 1,016 & 761 & 707 & 627 & 445 & 472 \\
\hline 7 (Fri) & 991 & 954 & 1,189 & 1,341 & 1,641 & 1,798 & 1,229 & 948 & 740 & 670 & 552 & 384 \\
\hline
\end{tabular}

$2400 \mathrm{SB}$

\begin{tabular}{|c|rrrrrrrrrrrrr|}
\hline Date(Day) & 00-01 & $\mathbf{0 1 - 0 2}$ & $\mathbf{0 2 - 0 3}$ & $\mathbf{0 3 - 0 4}$ & $\mathbf{0 4 - 0 5}$ & $\mathbf{0 5 - 0 6}$ & $\mathbf{0 6 - 0 7}$ & $\mathbf{0 7 - 0 8}$ & $\mathbf{0 8 - 0 9}$ & $\mathbf{0 9 - 1 0}$ & $\mathbf{1 0 - 1 1}$ & $\mathbf{1 1 - 1 2}$ \\
\hline $\mathbf{1 ( \text { Sat) }}$ & 328 & 262 & 205 & 192 & $\mathbf{2 2 5}$ & 293 & 424 & 527 & 774 & 957 & 1,224 & 1,280 \\
\hline 2(Sun) & 225 & 161 & 132 & 87 & 111 & 106 & 178 & 206 & 310 & 487 & 809 & 1,211 \\
\hline 3(Mon) & 260 & 191 & 143 & 156 & 150 & 342 & 895 & 1,364 & 1,020 & 855 & 753 & 767 \\
\hline 4(Tue) & 296 & 259 & 251 & 220 & 277 & 456 & 944 & 1,396 & 1,070 & 946 & 818 & 770 \\
\hline 5(Wed) & 295 & 236 & 218 & 242 & 256 & 457 & 960 & 1,458 & 1,062 & 868 & 796 & 765 \\
\hline 6(Thu) & 305 & 247 & 220 & 232 & 272 & 435 & 941 & 1,453 & 1,085 & 887 & 851 & 778 \\
\hline 7(Fri) & 292 & 284 & 247 & 236 & 269 & 464 & 975 & 1,388 & 1,127 & 879 & 871 & 860 \\
\hline Date(Day) & $12-13$ & $13-14$ & $14-15$ & $15-16$ & $16-17$ & $17-18$ & $18-19$ & $19-20$ & $20-21$ & $21-22$ & $22-23$ & $23-00$ \\
\hline 1(Sat) & 1,078 & 1,001 & 973 & 878 & 993 & 903 & 799 & 627 & 540 & 454 & 417 & 286 \\
\hline 2(Sun) & 1,209 & 1,068 & 947 & 1,022 & 999 & 1,067 & 1,106 & 906 & 781 & 670 & 526 & 334 \\
\hline 3(Mon) & 709 & 818 & 871 & 948 & 927 & 918 & 820 & 614 & 447 & 451 & 416 & 410 \\
\hline 4(Tue) & 756 & 826 & 879 & 939 & 1,006 & 1,037 & 759 & 592 & 475 & 422 & 377 & 377 \\
\hline 5(Wed) & 753 & 786 & 840 & 935 & 1,056 & 1,031 & 850 & 644 & 529 & 435 & 353 & 332 \\
\hline 6(Thu) & 762 & 836 & 876 & 977 & 1,081 & 1,104 & 908 & 676 & 586 & 474 & 459 & 419 \\
\hline 7(Fri) & 895 & 1,072 & 1,201 & 1,275 & 1,358 & 1,470 & 1,327 & 1,016 & 833 & 626 & 637 & 445 \\
\hline
\end{tabular}


Table 5.4 Sum of hourly volumes for both directions and both stations

\begin{tabular}{|c|c|c|c|c|c|c|c|c|c|c|c|c|}
\hline Date(Day) & 00-01 & $01-02$ & $02-03$ & $03-04$ & 04-05 & $05-06$ & $06-07$ & $07-08$ & 08-09 & $09-10$ & $10-11$ & $11-12$ \\
\hline 1 (Sat) & 1,491 & 1,162 & 828 & 801 & 838 & 1,162 & 1,865 & 2,595 & 3,471 & 4,193 & 4,898 & 5,239 \\
\hline 2 (Sun) & 1,227 & 773 & 584 & 487 & 438 & 484 & 844 & 1,081 & 1,689 & 2,507 & 3,457 & 4,564 \\
\hline 3(Mon) & 1,110 & 845 & 664 & 726 & 839 & 1,817 & 4,308 & 7,046 & 5,533 & 4,329 & 4291 & 4,554 \\
\hline 4(Tue) & 1,242 & 953 & 900 & 969 & 1,093 & 1,994 & 4,307 & 6,978 & 5,725 & 4,516 & 4,320 & 4,429 \\
\hline 5(Wed) & 1,256 & 965 & 951 & 945 & 1,096 & 1,987 & 4,318 & 7,165 & 5,687 & 4,574 & 4,419 & 4,549 \\
\hline 6(Thu) & 1,315 & 1,019 & 1,018 & 1,082 & 1,141 & 1,952 & 4,320 & 7,205 & 5,838 & 4,631 & 4,563 & 4,601 \\
\hline $7(\mathrm{Fri})$ & 1,362 & 1,116 & 1,046 & 990 & 1,184 & 2,026 & 4,329 & 6,868 & 5,684 & 4,728 & 4,871 & 5,171 \\
\hline Date(Day) & $12-13$ & $13-14$ & 14-15 & $15-16$ & $16-17$ & $17-18$ & $18-19$ & $19-20$ & $20-21$ & $21-22$ & $22-23$ & $23-00$ \\
\hline 1(Sat) & 5,216 & 4,988 & 5,224 & 5,201 & 5,104 & 4,940 & 4,415 & 3,539 & 2,937 & 2,705 & 2,353 & 1,825 \\
\hline 2 (Sun) & 5,198 & 5,381 & 5,474 & 5,800 & 6,139 & 5,900 & 5,295 & 4,194 & 3,453 & 2,662 & 2,138 & 1,502 \\
\hline 3(Mon) & 4,544 & 4,644 & 5,046 & 6,013 & 7,055 & 7,586 & 5,153 & 3,538 & 2,961 & 2,534 & 2,052 & 1,681 \\
\hline 4(Tue) & 4,635 & 4,788 & 5,071 & 6,285 & 7,263 & 7,430 & 4,242 & 3,085 & 3,006 & 2,611 & 2,143 & 1,672 \\
\hline 5(Wed) & 4,836 & 4,658 & 5,225 & 6,331 & 7,569 & 8,076 & 5,536 & 3,964 & 3,250 & 2,513 & 1,732 & 1,503 \\
\hline 6(Thu) & 4,973 & 5,064 & 5,520 & 6,548 & 7,475 & 8,176 & 5,655 & 4,027 & 3,383 & 2991 & 2,332 & 1,975 \\
\hline 7 (Fri) & 5,496 & 5,750 & 6,334 & 7,541 & 8,605 & 9,242 & 6,818 & 5,086 & 3,950 & 3,445 & 2,866 & 2,155 \\
\hline
\end{tabular}


Table 5.5 Peak period definitions and peak period factor calculations

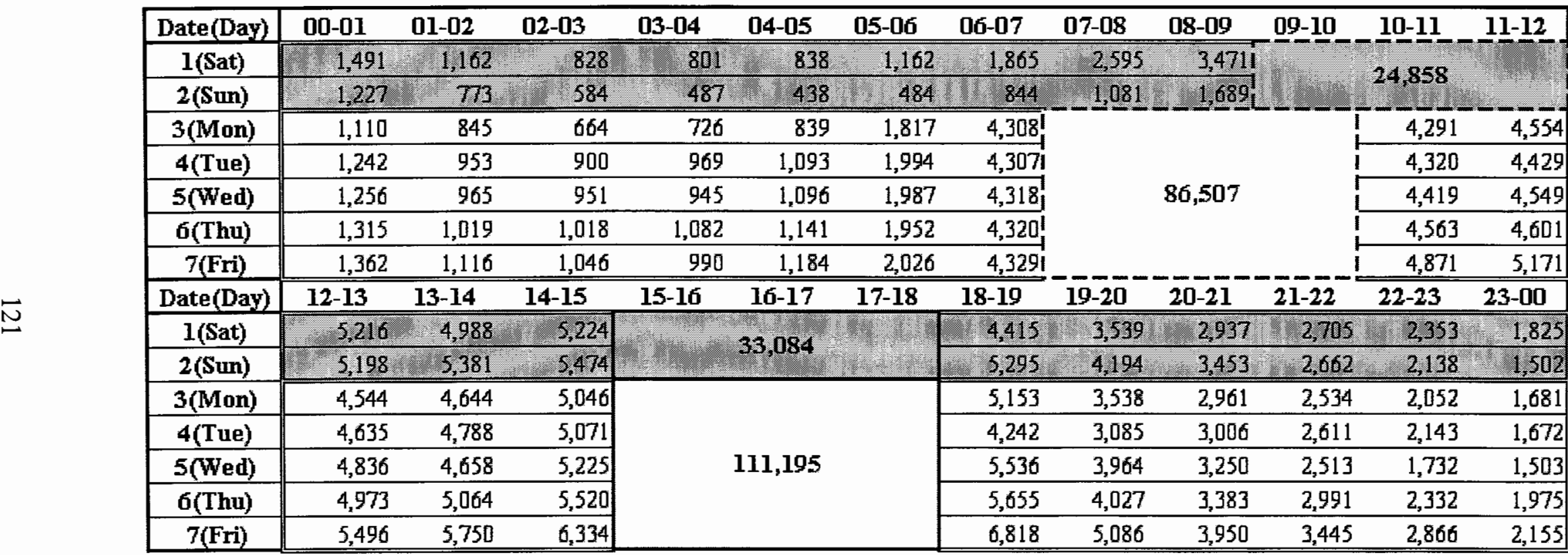

Sum of volumes

\begin{tabular}{|c|c|c|c|c|}
\hline daytye & AM-peak & PM-peak & Off-peak & Total \\
\hline weekend & 24,858 & $\mathbf{3 3 , 0 8 4}$ & $\mathbf{9 0 , 3 1 9}$ & 148,261 \\
\hline wreekday & $\mathbf{8 6}, 507$ & $\mathbf{1 1 1 , 1 9 5}$ & $\mathbf{2 7 7 , 3 9 6}$ & 475,098 \\
\hline
\end{tabular}

peak factor

\begin{tabular}{|c|c|c|c|}
\hline daytype & AM-peak & PM-peak & Off-peak \\
\hline weekend & 0.056 & 0.074 & 0.034 \\
\hline weekday & 0.061 & 0.078 & 0.032 \\
\hline
\end{tabular}


Table 5.6 Peak period definitions based on a single ATR station

Sum of valumes for both directions (Station 1420)

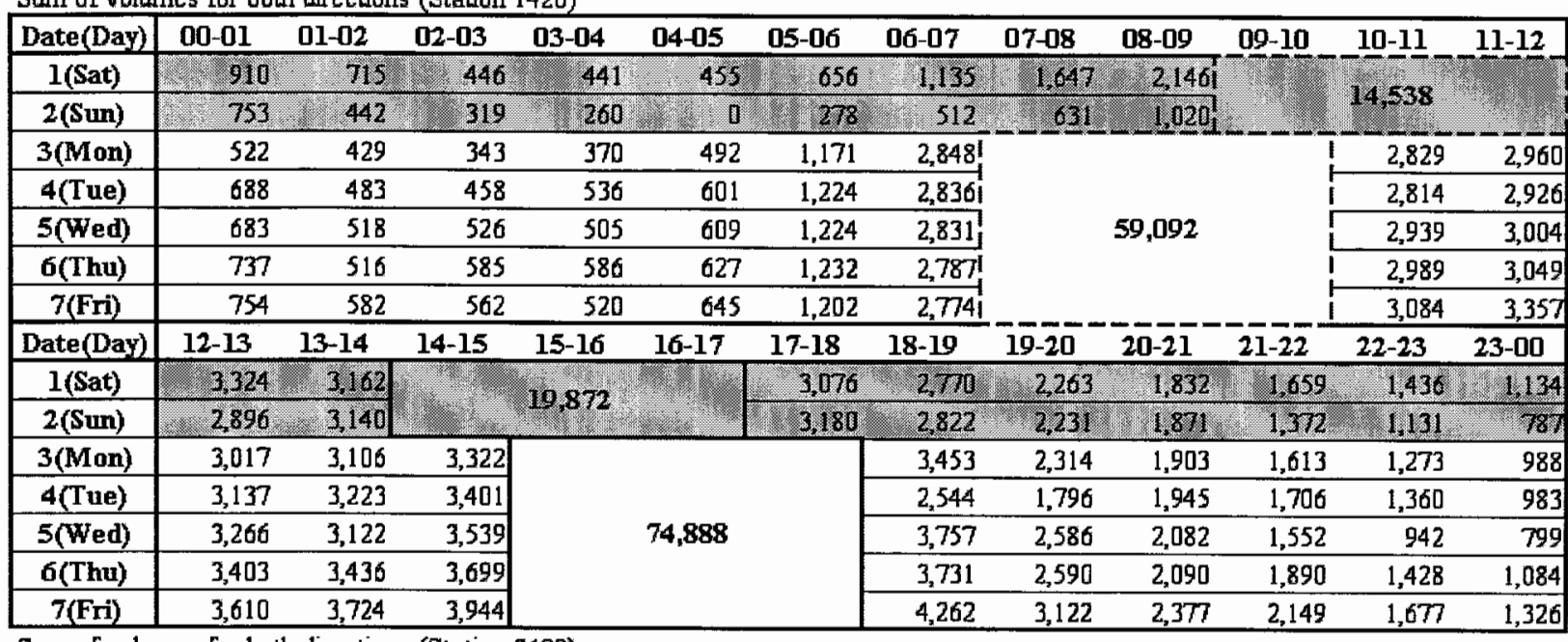

Sum af volumes for both directions (Station 2400)

\begin{tabular}{|c|c|c|c|c|c|c|c|c|c|c|c|c|}
\hline Date(Day) & $00-01$ & $01-02$ & $02-03$ & 03-04 & $04-05$ & $05-06$ & $06-07$ & $07-08$ & 08-09 & $09-10$ & $10-11$ & $11-12$ \\
\hline l(Sat) & 581 & 1.447 & 382 & $=360$ & 383 & 8.506 & 730 & 948 & 1,3251 & & \multirow{2}{*}{19,320} & \\
\hline 2 (Sun) & 474. & 1331 & $\sqrt{265}$ & .22 & 20 & 206 & 32 & 450 & & & & \\
\hline 3 (Mon) & 588 & 416 & 321 & 356 & 347 & 646 & 1,460 & & \multirow{5}{*}{27,415} & & 1,462 & 1,594 \\
\hline 4(Tue) & 554 & 470 & 442 & 433 & 492 & 770 & 1,471 & & & & 1,506 & 1,503 \\
\hline 5(Wed) & 573 & 447 & 425 & 440 & 487 & 763 & 1,487 & & & & 1,480 & 1,545 \\
\hline 6(Thu) & 578 & 503 & 433 & 496 & 514 & 720 & 1,533 & & & & 1,574 & 1,552 \\
\hline 7 (Fri) & 608 & 534 & 484 & 470 & 539 & 824 & 1,555 & & & & 1,787 & 1,814 \\
\hline Date(Day) & $12-13$ & $13-14$ & $14-15$ & $15-16$ & $16-17$ & $17-18$ & $18-19$ & $19-20$ & $20-21$ & $21-22$ & $22-23$ & 23-00 \\
\hline 1(Sat) & 1.892 & 1,826 & 1,932 & \multirow{2}{*}{13580} & \multirow{2}{*}{18.580} & & $=1.645$ & 1.276 & 1.105 & 1046 & 917 & $=691$ \\
\hline 2 (Sun) & 2,302 & 2,241 & 2172 & & & & 2473 & 1963 & 1,582 & 1.290 & 1.007 & 715 \\
\hline 3(Mon) & 1,527 & 1,538 & 1,724 & & \multirow{5}{*}{36,307} & & 1,700 & 1,224 & 1,058 & 921 & 779 & 693 \\
\hline 4(Tue) & 1,498 & 1,565 & 1,670 & & & & 1,698 & 1,289 & 1,061 & 905 & 783 & 689 \\
\hline 5(Wed) & 1,570 & 1,536 & 1,686 & & & & 1,779 & 1,378 & 1,168 & 961 & 790 & 704 \\
\hline 6(Thu) & 1,570 & 1,628 & 1,821 & & & & 1,924 & 1,437 & 1,293 & 1,101 & 904 & 891 \\
\hline 7 (Fri) & 1,886 & 2,026 & 2,390 & & & & 2,556 & 1,964 & 1,573 & 1,296 & 1,189 & 829 \\
\hline
\end{tabular}


Table 5.7(a) Sum of volumes for each direction for davs helonging to the same dav tvne

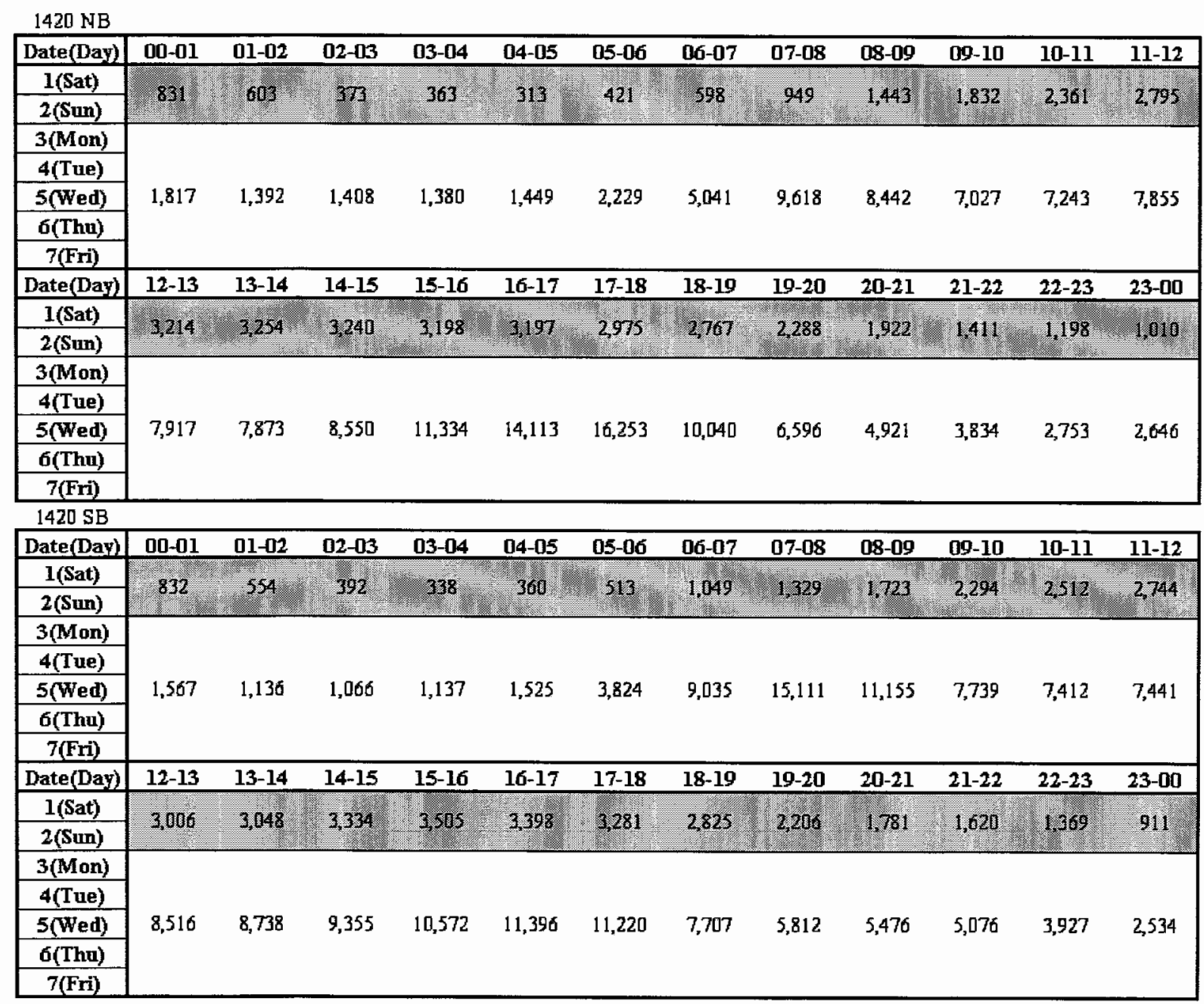

2. weekends $\square$ weekdays 
Table 5.7(b) Sum of volumes for each direction for days belonging to the same day type

\begin{tabular}{|c|c|c|c|c|c|c|c|c|c|c|c|c|}
\hline Date(Day) & 00-01 & $01-02$ & $02-03$ & 03-04 & $04-05$ & $05-06$ & $06-07$ & 07-08 & $08-09$ & 09-10 & $10-11$ & 11-12 \\
\hline \begin{tabular}{l|} 
(Sat) \\
2 (Sun)
\end{tabular} & $\sin 2$ & 355 & 310 & 308 & 267 & 313 & 460 & 665 & 910 & 1,130 & 1,49 & 1,773 \\
\hline 3(Mon) & \multirow{4}{*}{1,453} & \multirow{4}{*}{1,153} & \multirow{4}{*}{1,026} & \multirow{4}{*}{1,109} & \multirow{4}{*}{1,155} & \multirow{4}{*}{1,569} & \multirow{4}{*}{2,791} & \multirow{4}{*}{3,474} & \multirow{4}{*}{3,506} & \multirow{4}{*}{3,577} & \multirow{4}{*}{3,720} & \multirow{4}{*}{4,068} \\
\hline 4(Tue) & & & & & & & & & & & & \\
\hline 5(Wed) & & & & & & & & & & & & \\
\hline $\begin{array}{c}6 \text { (Thu) } \\
7 \text { (Fri) } \\
\end{array}$ & & & & & & & & & & & & \\
\hline Date(Day) & $12-13$ & $13-14$ & 14-15 & 15-16 & $16-17$ & $17-18$ & $18-19$ & $19-20$ & $20-21$ & $21-22$ & $22-23$ & 23-00 \\
\hline $\begin{array}{l}\text { (Sat) } \\
2 \text { (Sun) } \\
\end{array}$ & 1,907 & 1998 & 2284 & 2,398 & 2,656 & 2,614 & 2,213 & 1,706 & 1,360 & 1.212 & 981 & 786 \\
\hline 3(Mon) & & & & & & & & & & & & \\
\hline 4(Tue) & & & & & & & & & & & & \\
\hline 5(Wed) & 4,176 & 3,955 & 4,624 & 5,738 & 7,030 & 7,477 & 4,993 & 3,750 & 3,283 & 2,776 & 2,203 & 1,823 \\
\hline $\begin{array}{c}6 \text { (Thu) } \\
7 \text { (Fri) } \\
\end{array}$ & & & & & & & & & & & & \\
\hline $2400 \mathrm{SB}$ & & & & & & & & & & & & \\
\hline Date(Day) & 00-01 & $01-02$ & $02-03$ & 03-04 & 04-05 & 05-06 & 06-07 & 07-08 & 08-09 & 09-10 & $10-11$ & $11-12$ \\
\hline \begin{tabular}{|l|}
1 (Sat) \\
2 (Sun)
\end{tabular} & 583 & 423 & 337 & 279 & 336 & 3998 & 6112 & T33 & 1,084 & 1,444 & 2087 & 2,491 \\
\hline 3(Mon) & & & & & & & & & & & & \\
\hline 4(Tue) & & & & & & & & & & & & \\
\hline 5(Wed) & 1,448 & 1,217 & 1,079 & 1,086 & 1,224 & 2,154 & 4,715 & 7,059 & 5,364 & 4,435 & 4,089 & 3,940 \\
\hline $\begin{array}{c}\text { 6(Thu) } \\
7 \text { (Fri) } \\
\end{array}$ & & & & & & & & & & & & \\
\hline Date(Day) & $12-13$ & $13-14$ & $14-15$ & $15-16$ & $16-17$ & $17-18$ & $18-19$ & $19-20$ & $20-21$ & $21-22$ & $22-23$ & $23-00$ \\
\hline $\begin{array}{l}1 \text { (Sat) } \\
2(\text { Sun) }\end{array}$ & 2287 & 2869 & 1,920 & 1,900 & 9.992 & 1,970 & 1,905 & 1,53 & 1,321 & 1.124 & 94 & 620 \\
\hline 3(Mon) & & & & & & & & & & & & \\
\hline 4(Tue) & & & & & & & & & & & & \\
\hline 5(Wed) & 3,875 & 4,338 & 4,667 & 5,074 & 5,428 & 5,560 & 4,664 & 3,542 & 2,870 & 2,408 & 2,242 & 1,983 \\
\hline G(Thu) & & & & & & & & & & & & \\
\hline 7(Fri) & & & & & & & & & & & & \\
\hline
\end{tabular}

Weekends $\square$ weekdays 
Table 5.8 Larger sum of volumes between two directions for days belonging to the same day type

\begin{tabular}{|c|c|c|c|c|c|c|c|c|c|c|c|c|}
\hline Date(Day) & $00-01$ & $01-02$ & $02-03$ & 03-04 & $04-05$ & 05-06 & 06-07 & $07-08$ & 08-09 & 09-10 & $10-11$ & 11-12 \\
\hline $\begin{array}{l}1 \text { (Sat) } \\
2 \text { (Sun) }\end{array}$ & 832 & 603. & 392 & 363 & 360 & 513 & 1,049 & 1,329 & 1,723 & 2,294 & 23512 & 2,795 \\
\hline 3(Mon) & \multirow{3}{*}{1,817} & \multirow{3}{*}{1,392} & \multirow{3}{*}{1,408} & \multirow{3}{*}{1,380} & \multirow{3}{*}{1,525} & \multirow{3}{*}{3,824} & \multirow{3}{*}{9,035} & \multirow{3}{*}{15,111} & \multirow{3}{*}{11,155} & \multirow{3}{*}{7,739} & \multirow{3}{*}{7,412} & \multirow{3}{*}{7,855} \\
\hline $\begin{array}{l}4 \text { (Tue) } \\
5 \text { (Wed) }\end{array}$ & & & & & & & & & & & & \\
\hline$\frac{6 \text { (Thu) }}{7(\mathrm{Fri})}$ & & & & & & & & & & & & \\
\hline Date(Day) & $12-13$ & 13-14 & $14-15$ & 15-16 & $16-17$ & $17-18$ & 18-19 & $19-20$ & $20-21$ & $21-22$ & $22-23$ & $23-00$ \\
\hline$\frac{1 \text { (Sat) }}{2 \text { (Sun) }}$ & 3,214 & 3.254 & 3334 & 3.505 & 3,398 & 1281 & 2,825 & 2,288 & 1,922 & 1,620 & 1,369 & 1010 \\
\hline 3(Mon) & \multirow{5}{*}{8,516} & \multirow{5}{*}{8,738} & \multirow{5}{*}{9,355} & \multirow{5}{*}{11,334} & \multirow{5}{*}{14,113} & \multirow{5}{*}{16,253} & \multirow{5}{*}{10,040} & \multirow{5}{*}{6,596} & \multirow{5}{*}{5,476} & \multirow{5}{*}{5,076} & \multirow{5}{*}{3,927} & \multirow{5}{*}{2,646} \\
\hline 4(Tue) & & & & & & & & & & & & \\
\hline 5 (Wed) & & & & & & & & & & & & \\
\hline 6(Thu) & & & & & & & & & & & & \\
\hline$\frac{7 \text { (Fri) }}{2400}$ & & & & & & & & & & & & \\
\hline \multicolumn{13}{|l|}{2400} \\
\hline Date(Day) & 00-01 & 01-02 & $02-03$ & $03-04$ & 04-05 & $05-06$ & 06-07 & $07-08$ & 08-09 & 09-10 & 10-11 & 11-12 \\
\hline $\begin{array}{l}\text { (Sat) } \\
2 \text { (Sun) }\end{array}$ & 553 & 423 & 337 & 308 & 336 & 399 & 602 & 733 & 1.084 & 1,444 & 2,033 & 2,491 \\
\hline 3(Mon) & \multirow{4}{*}{1,453} & \multirow{4}{*}{1,217} & \multirow{4}{*}{1,079} & \multirow{4}{*}{1,109} & \multirow{4}{*}{1,224} & & & & & & & \\
\hline 4(Tue) & & & & & & & & & & & & 4068 \\
\hline$\frac{5 \text { (Wed) }}{6 \text { (Thu) }}$ & & & & & & 2,154 & 4,715 & 7,059 & 5,364 & 4,435 & 4,089 & 4,068 \\
\hline 7 (Fri) & & & & & & & & & & & & \\
\hline Date(Day) & $12-13$ & 13-14 & 14-15 & $15-16$ & $16-17$ & 17-18 & $18-19$ & $19-20$ & 20-21 & $21-22$ & $22-23$ & $23-00$ \\
\hline $\begin{array}{l}1 \text { (Sat) } \\
2 \text { (Sun) }\end{array}$ & 2,287 & 2,069 & 2,204 & 2,398 & 2,656 & 2614 & 2,213 & 1.706 & 1,366 & 1,212 & 981 & 786 \\
\hline 3(Mon) & & & & & & & & & & & & \\
\hline 4(Tue) & & & & & & & & & & & & \\
\hline 5(Wed) & 4,176 & 4,338 & 4,667 & 5,738 & 7,030 & 7,477 & 4,993 & 3,750 & 3,283 & 2,776 & 2,242 & 1,983 \\
\hline 6(Thu) & & & & & & & & & & & & \\
\hline 7 (Fri) & & & & & & & & & & & & \\
\hline
\end{tabular}

weekends weekdays 
Table 5.9 Sum of larger sum of volumes between two directions for different day types for all stations belonging to the same road class

\begin{tabular}{|c|c|c|c|c|c|c|c|c|c|c|c|c|}
\hline Date(Day) & 00-01 & $01-02$ & $02-03$ & 03-04 & 04-05 & $05-06$ & $06-07$ & $07-08$ & 08-09 & $09-10$ & 10-11 & 11-12 \\
\hline 1 (Sat) & 1,385 & 1,026 & 729 & 671 & 696 & 912 & 1,651 & 2,062 & 2,807 & 3,738 & 4,545 & 5,286 \\
\hline 2 (Sun) & 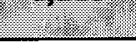 & 32 & & & & & & & & & & \\
\hline 3(Mon) & \multirow{5}{*}{3,270} & \multirow{5}{*}{2,609} & \multirow{5}{*}{2,487} & \multirow{5}{*}{2,489} & \multirow{5}{*}{2,749} & \multirow{5}{*}{5,978} & \multirow{5}{*}{13,750} & \multirow{5}{*}{22,170} & \multirow{5}{*}{16,519} & \multirow{5}{*}{12,174} & \multirow{5}{*}{11,501} & \multirow{5}{*}{11,923} \\
\hline 4(Tue) & & & & & & & & & & & & \\
\hline 5(Wed) & & & & & & & & & & & & \\
\hline 6(Thu) & & & & & & & & & & & & \\
\hline 7 (Fri) & & & & & & & & & & & & \\
\hline Date(Day) & $12-13$ & $13-14$ & 14-15 & 15-16 & $16-17$ & $17-18$ & $18-19$ & $19-20$ & $20-21$ & $21-22$ & $22-23$ & 23-00 \\
\hline l(Sat) & \multirow{2}{*}{5,501} & \multirow{2}{*}{5,323} & \multirow{2}{*}{5,538} & \multirow{2}{*}{5,903} & \multirow{2}{*}{6,054} & \multirow{2}{*}{5,895} & \multirow{2}{*}{5,038} & \multirow{2}{*}{3,994} & \multirow{2}{*}{3,288} & \multirow{2}{*}{2,832} & \multirow{2}{*}{2,350} & \multirow{2}{*}{1,796} \\
\hline 2(Sun) & & & & & & & & & & & & \\
\hline 3(Mon) & \multirow{5}{*}{12,692} & \multirow{5}{*}{13,076} & \multirow{5}{*}{14,022} & \multirow{5}{*}{17,072} & \multirow{5}{*}{21,143} & \multirow{5}{*}{23,730} & \multirow{5}{*}{15,033} & \multirow{5}{*}{10,346} & \multirow{5}{*}{8,759} & \multirow{5}{*}{7,852} & \multirow{5}{*}{6,169} & \\
\hline 4(Tue) & & & & & & & & & & & & \\
\hline 5(Wed) & & & & & & & & & & & & 4,629 \\
\hline 6(Thu) & & & & & & & & & & & & \\
\hline 7 (Fri) & & & & & & & & & & & & \\
\hline
\end{tabular}

प weekends $\quad$ weekdays


Table 5.10 Directionality calculation

\begin{tabular}{|c|c|c|c|c|c|c|c|c|c|c|c|c|}
\hline Date(Day) & 00-01 & 01-02 & $02-03$ & $03-04$ & 04-05 & 05-06 & $06-07$ & $07-08$ & 08-09 & 09-10 & 10-11 & $11-12$ \\
\hline l(Sat) & 1,385 & 1.026 & 729 & 671 & 696 & 912 & 1,65 & 2,062 & 2,807 & & 13,569 & \\
\hline 2 (Sun) & & & & & & & & & 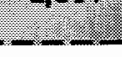 & & & \\
\hline 3(Mon) & \multirow{5}{*}{3,270} & \multirow{5}{*}{2,609} & \multirow{5}{*}{2,487} & \multirow{5}{*}{2,489} & \multirow{5}{*}{2,749} & \multirow{5}{*}{5,978} & \multirow{5}{*}{13,750} & & \multirow{5}{*}{50,863} & & \multirow{5}{*}{11,501} & \multirow{5}{*}{11,923} \\
\hline 4(Tue) & & & & & & & & & & & & \\
\hline 5(Wed) & & & & & & & & & & & & \\
\hline 6(Thu) & & & & & & & & & & & & \\
\hline 7 (Fri) & & & & & & & & & & & & \\
\hline Date(Day) & $12-13$ & $13-14$ & $14-15$ & $15-16$ & $16-17$ & $17-18$ & $18-19$ & $19-20$ & $20-21$ & $21-22$ & $22-23$ & 23-00 \\
\hline 1 (Sat) & \multirow{2}{*}{5,501} & \multirow{2}{*}{5,323} & \multirow{2}{*}{5,538} & \multirow{2}{*}{\multicolumn{3}{|c|}{17,852}} & \multirow{2}{*}{5,038} & \multirow{2}{*}{3,994} & \multirow{2}{*}{3,288} & \multirow{2}{*}{2.832} & \multirow{2}{*}{$\begin{array}{r}2,350 \\
\end{array}$} & \multirow{2}{*}{1,796} \\
\hline 2(Sun) & & & & & & & & & & & & \\
\hline 3(Mon) & \multirow{5}{*}{12,692} & \multirow{5}{*}{13,076} & \multirow{5}{*}{14,022} & \multirow{5}{*}{\multicolumn{3}{|c|}{61,945}} & \multirow{5}{*}{15,033} & \multirow{5}{*}{10,346} & \multirow{5}{*}{8,759} & \multirow{5}{*}{7,852} & \multirow{5}{*}{6,169} & \multirow{5}{*}{4,629} \\
\hline 4(Tue) & & & & & & & & & & & & \\
\hline 5(Wed) & & & & & & & & & & & & \\
\hline 6(Thu) & & & & & & & & & & & & \\
\hline 7 (Fri) & & & & & & & & & & & & \\
\hline
\end{tabular}

Sum of larger volumes between two directions

\begin{tabular}{|c|c|c|c|}
\hline daytype & AM-peak & PM-peak & Off-peak \\
\hline weekend & 13,569 & 17,852 & 47,599 \\
\hline weekday & 50,863 & 61,945 & 149,334 \\
\hline
\end{tabular}

Directionality
\begin{tabular}{|c|c|c|c|}
\hline daytye & AM-peak & PM-peak & Off-peak \\
\hline weekend & $54.6 \%$ & $54.0 \%$ & $52.7 \%$ \\
\hline weekday & $\mathbf{5 8 . 8 \%}$ & $\mathbf{5 5 . 7 \%}$ & $\mathbf{5 3 . 8 \%}$ \\
\hline
\end{tabular}


Table 5.11 Annual growth factors by functional class from 1990 to 1999 in Indiana State

\begin{tabular}{|l|c|c|c|c|c|c|c|c|c|}
\hline Year bases & 1990 & 1991 & 1992 & 1993 & 1994 & 1995 & 1996 & 1997 & 1998 \\
to & to & to & to & to & to & $\begin{array}{c}\text { to } \\
\text { to }\end{array}$ & $\begin{array}{c}19 \\
\text { to }\end{array}$ \\
\hline Urban interstate & 0.993 & 1.074 & 1.072 & 1.032 & 1.103 & 1.047 & 1.066 & 1.038 & 1.038 \\
\hline Urban others & 1.032 & 1.036 & 1.011 & 0.982 & 1.034 & 1.014 & 1.082 & 1.023 & 1.016 \\
\hline Rural interstate & 1.024 & 1.051 & 1.021 & 1.078 & 1.004 & 1.021 & 1.070 & 1.036 & 1.033 \\
\hline Rural arterials & 0.997 & 1.045 & 1.019 & 1.028 & 1.035 & 0.998 & 1.063 & 1.047 & 1.002 \\
\hline Rural collectors & 1.034 & 1.045 & 1.015 & 1.025 & 1.094 & 1.052 & 1.038 & 0.999 & 1.031 \\
and locals & & & & & & & & & \\
\hline
\end{tabular}

Source: Indiana Department of Transportation, Roadway Management Division 
Table 5.12 Seasonal Adjustment Factors by Functional Class: 1994-1998

Urban Interstates, Freeways and Expressways

\begin{tabular}{ccccccccccccc}
\hline & Jan & Feb & Mar & Apr & May & Jun & Jul & Aug & Sep & Oct & Nov & Dec \\
\hline 1998 & 1.190 & 1.109 & 1.100 & 1.007 & 1.013 & 0.980 & 0.957 & 0.935 & 0.959 & 0.940 & 0.958 & 0.973 \\
1997 & 1.177 & 1.081 & 1.026 & 0.959 & 0.981 & 0.952 & 0.926 & 0.939 & 1.002 & 1.010 & 1.034 & 1.073 \\
1996 & 1.207 & 1.035 & 1.079 & 0.987 & 1.016 & 0.958 & 0.937 & 0.911 & 0.974 & 0.952 & 0.980 & 1.041 \\
1995 & 1.108 & 1.108 & 1.012 & 0.982 & 0.995 & 0.941 & 0.933 & 0.982 & 0.999 & 0.971 & 0.973 & 1.029 \\
1994 & 1.218 & 1.090 & 1.015 & 0.975 & 0.941 & 0.920 & 0.933 & 0.901 & 0.946 & 0.923 & 0.989 & 0.937 \\
\hline AVG & $\mathbf{1 . 1 8 0}$ & $\mathbf{1 . 0 8 5}$ & $\mathbf{1 . 0 4 6}$ & $\mathbf{0 . 9 8 2}$ & $\mathbf{0 . 9 8 9}$ & $\mathbf{0 . 9 5 0}$ & $\mathbf{0 . 9 3 7}$ & $\mathbf{0 . 9 3 3}$ & $\mathbf{0 . 9 7 6}$ & $\mathbf{0 . 9 5 9}$ & $\mathbf{0 . 9 8 7}$ & $\mathbf{1 . 0 1 1}$ \\
\hline
\end{tabular}

Urban - Principal Arterials, Minor Arterials, Collectors and Locals

\begin{tabular}{ccccccccccccc}
\hline & Jan & Feb & Mar & Apr & May & Jun & Jul & Aug & Sep & Oct & Nov & Dec \\
\hline 1998 & 1.093 & 1.035 & 1.018 & 1.029 & 0.963 & 0.946 & 0.947 & 0.975 & 1.008 & 0.997 & 1.041 & 1.019 \\
1997 & 1.143 & 1.045 & 0.997 & 1.049 & 0.980 & 0.941 & 0.991 & 0.968 & 1.022 & 0.986 & 0.994 & 1.003 \\
1996 & 1.110 & 0.983 & 1.055 & 0.989 & 0.975 & 0.958 & 0.935 & 1.077 & 1.043 & 0.973 & 1.016 & 1.029 \\
1995 & 1.110 & 1.080 & 1.038 & 1.020 & 0.965 & 0.953 & 0.976 & 0.971 & 0.989 & 0.974 & 0.907 & 1.055 \\
1994 & 1.104 & 1.008 & 0.977 & 0.944 & 0.928 & 0.932 & 0.955 & 0.934 & 0.939 & 0.920 & 0.958 & 0.955 \\
\hline AVG & $\mathbf{1 . 1 1 2}$ & $\mathbf{1 . 0 3 0}$ & $\mathbf{1 . 0 1 7}$ & $\mathbf{1 . 0 0 6}$ & $\mathbf{0 . 9 6 2}$ & $\mathbf{0 . 9 4 6}$ & $\mathbf{0 . 9 6 1}$ & $\mathbf{0 . 9 8 5}$ & $\mathbf{1 . 0 0 0}$ & $\mathbf{0 . 9 7 0}$ & $\mathbf{0 . 9 8 3}$ & $\mathbf{1 . 0 1 2}$ \\
\hline
\end{tabular}

Rural Interstates

\begin{tabular}{ccccccccccccc}
\hline & Jan & Feb & Mar & Apr & May & Jun & Jul & Aug & Sep & Oct & Nov & Dec \\
\hline 1998 & 1.203 & 1.139 & 1.074 & 0.986 & 0.968 & 0.953 & 0.895 & 0.907 & 0.983 & 0.987 & 0.993 & 1.028 \\
1997 & 1.128 & 1.042 & 0.951 & 1.117 & 1.014 & 1.007 & 0.922 & 0.938 & 1.036 & 1.011 & 1.032 & 1.124 \\
1996 & 1.301 & 1.115 & 1.103 & 1.025 & 0.992 & 0.921 & 0.833 & 0.854 & 0.952 & 0.985 & 1.020 & 1.047 \\
1995 & 1.141 & 1.173 & 1.021 & 0.965 & 0.975 & 0.930 & 0.930 & 0.948 & 0.980 & 0.966 & 0.987 & 1.083 \\
1994 & 1.423 & 1.234 & 1.115 & 1.079 & 1.016 & 0.985 & 0.922 & 0.915 & 1.016 & 1.023 & 1.018 & 0.991 \\
\hline AVG & $\mathbf{1 . 2 3 9}$ & $\mathbf{1 . 1 4 1}$ & $\mathbf{1 . 0 5 3}$ & $\mathbf{1 . 0 3 4}$ & $\mathbf{0 . 9 9 3}$ & $\mathbf{0 . 9 5 9}$ & $\mathbf{0 . 9 0 0}$ & $\mathbf{0 . 9 1 2}$ & $\mathbf{0 . 9 9 3}$ & $\mathbf{0 . 9 9 4}$ & $\mathbf{1 . 0 1 0}$ & $\mathbf{1 . 0 5 5}$ \\
\hline
\end{tabular}

Rural - Principal Arterials and Minor Arterials

\begin{tabular}{ccccccccccccc}
\hline & Jan & Feb & Mar & Apr & May & Jun & Jul & Aug & Sep & Oct & Nov & Dec \\
\hline 1998 & 1.161 & 1.095 & 1.091 & 1.014 & 0.983 & 0.943 & 0.919 & 0.934 & 0.962 & 0.958 & 1.001 & 1.019 \\
1997 & 1.140 & 1.031 & 0.985 & 1.018 & 1.022 & 0.991 & 0.944 & 0.958 & 1.002 & 0.996 & 1.036 & 1.087 \\
1996 & 1.254 & 1.045 & 1.154 & 1.057 & 0.985 & 0.943 & 0.891 & 0.901 & 0.943 & 0.937 & 1.007 & 1.073 \\
1995 & 1.138 & 1.107 & 1.056 & 0.988 & 0.961 & 0.918 & 0.902 & 0.920 & 0.942 & 0.973 & 1.034 & 1.139 \\
1994 & 1.197 & 1.136 & 1.081 & 1.002 & 0.953 & 0.900 & 0.908 & 0.890 & 0.941 & 0.963 & 1.028 & 0.994 \\
\hline AVG & $\mathbf{1 . 1 7 8}$ & $\mathbf{1 . 0 8 3}$ & $\mathbf{1 . 0 7 3}$ & $\mathbf{1 . 0 1 6}$ & $\mathbf{0 . 9 8 1}$ & $\mathbf{0 . 9 3 9}$ & $\mathbf{0 . 9 1 3}$ & $\mathbf{0 . 9 2 1}$ & $\mathbf{0 . 9 5 8}$ & $\mathbf{0 . 9 6 5}$ & $\mathbf{1 . 0 2 1}$ & $\mathbf{1 . 0 6 2}$ \\
\hline
\end{tabular}

Rural - Major Collectors, Minor Collectors and Locals

\begin{tabular}{ccccccccccccc}
\hline & Jan & Feb & Mar & Apr & May & Jun & Jul & Aug & Sep & Oct & Nov & Dec \\
\hline 1998 & 1.104 & 1.072 & 1.070 & 0.988 & 0.961 & 0.935 & 0.943 & 0.978 & 0.992 & 0.962 & 1.019 & 1.050 \\
1997 & 1.187 & 1.079 & 1.030 & 0.961 & 0.974 & 0.935 & 0.916 & 0.959 & 0.997 & 0.980 & 1.026 & 1.071 \\
1996 & 1.179 & 1.019 & 1.109 & 0.992 & 0.990 & 0.956 & 0.943 & 0.996 & 0.976 & 0.959 & 1.009 & 1.072 \\
1995 & 1.154 & 1.126 & 1.073 & 1.010 & 0.971 & 0.914 & 0.906 & 0.923 & 0.976 & 0.949 & 0.961 & 1.119 \\
1994 & 1.210 & 1.082 & 1.009 & 0.966 & 0.929 & 0.906 & 0.915 & 0.945 & 0.958 & 0.951 & 0.982 & 0.977 \\
\hline AVG & $\mathbf{1 . 1 6 7}$ & $\mathbf{1 . 0 7 6}$ & $\mathbf{1 . 0 5 8}$ & $\mathbf{0 . 9 8 3}$ & $\mathbf{0 . 9 6 5}$ & $\mathbf{0 . 9 2 9}$ & $\mathbf{0 . 9 2 5}$ & $\mathbf{0 . 9 6 0}$ & $\mathbf{0 . 9 8 0}$ & $\mathbf{0 . 9 6 0}$ & $\mathbf{0 . 9 9 9}$ & $\mathbf{1 . 0 5 8}$ \\
\hline
\end{tabular}

Source: Indiana Department of Transportation, Roadway Management Division 
Table 5.13 Comparisons of annual growth factors based on the TMG with the ones adopted by INDOT

\begin{tabular}{|c|c|c|}
\hline Road class & $\begin{array}{c}\text { Growth factor } \\
\text { based on TMG }\end{array}$ & $\begin{array}{c}\text { Growth factor } \\
\text { adopted by INDOT }\end{array}$ \\
\hline Urban Interstate & 1.11 & 1.038 \\
\hline Urban others & 1.04 & 1.023 \\
\hline Rural interstate & 1.06 & 1.036 \\
\hline Rural others & 1.06 & $1.023 *$ \\
\hline
\end{tabular}

* from the average of 1.047 for rural arterials, and 0.999 for rural collectors and locals 
Table 5.14 Stability analysis on the number of iterations in UE and SUE assignment models

\begin{tabular}{|c|c|c|c|c|c|c|}
\hline \multirow[b]{2}{*}{$\begin{array}{l}\text { Number } \\
\text { of } \\
\text { iterations }\end{array}$} & \multicolumn{3}{|c|}{ UE } & \multicolumn{3}{|c|}{ SUE } \\
\hline & $\Sigma|\mathrm{A}-\mathrm{B}|$ & $\Sigma(\mathrm{A}-\mathrm{B})^{2} / \mathrm{A}$ & $\begin{array}{c}\text { Number of } \\
\text { zero-flow } \\
\text { links }\end{array}$ & $\Sigma|\mathrm{A}-\mathrm{B}|$ & $\Sigma(\mathrm{A}-\mathrm{B})^{2} / \mathrm{A}$ & \begin{tabular}{|c|} 
Number of \\
zero-flow \\
links
\end{tabular} \\
\hline 20 & $46,238,172$ & $28,137,863$ & 535 & $54,917,538$ & $42,787,190$ & 132 \\
\hline 25 & $46,238,172$ & $28,137,863$ & 535 & $54,545,112$ & $42,573,536$ & 132 \\
\hline 30 & - & - & - & $54,127,092$ & $42,500,197$ & 132 \\
\hline 35 & - & - & - & $54,140,713$ & $42,286,770$ & 132 \\
\hline 40 & - & - & - & $54,065,832$ & $42,171,622$ & 132 \\
\hline 45 & - & - & - & $53,919,335$ & $42,018,432$ & 132 \\
\hline 50 & - & - & - & $53,912,598$ & $42,163,093$ & 132 \\
\hline 60 & - & - & - & $53,632,699$ & $41,362,841$ & 132 \\
\hline 70 & - & - & - & $53,556,660$ & $41,217,510$ & 132 \\
\hline 80 & - & - & - & $53,527,476$ & $41,234,556$ & 132 \\
\hline 150 & - & - & - & $53,503,385$ & $40,989,211$ & 123 \\
\hline 200 & - & - & - & $53,462,769$ & $40,759,482$ & 123 \\
\hline
\end{tabular}


Table 5.15 Workzone capacity formula

\begin{tabular}{|c|c|c|c|c|c|}
\hline \multirow{2}{*}{\begin{tabular}{c} 
Normal number of $\begin{array}{c}\text { Ines in one } \\
\text { direction }\end{array}$ \\
\cline { 2 - 6 }
\end{tabular}} & \multicolumn{5}{|c|}{$\begin{array}{c}\text { Open lanes through work zone in } \\
\text { one direction }\end{array}$} \\
\hline & 1 & 2 & 3 & 4 & 5 \\
\hline 2 & 1460 & & & & \\
\hline 3 & 1370 & 1600 & & & \\
\hline 4 & 1200 & 1580 & 1560 & & \\
\hline 5 & 1200 & 1460 & 1500 & 1550 & \\
\hline 6 & 1200 & 1400 & 1500 & 1550 & 1580 \\
\hline & & Slope Term (b) & \\
\hline & 1 & 2 & 3 & 4 & 5 \\
\hline 2 & 2.13 & & & & \\
\hline 3 & 4.05 & 1.81 & & & \\
\hline 4 & 0.00 & 1.60 & 0.57 & & \\
\hline 5 & 0.00 & 1.46 & 0.00 & 0.00 & \\
\hline 6 & 0.00 & 0.00 & 0.00 & 0.00 & 0.00 \\
\hline
\end{tabular}

Workzone Capacity $=\mathrm{a}-(\mathrm{b} \times \mathrm{CERF})$

where CERF is the capacity estimate risk factor.

Source: Memmott and Dudek (1982) 
Table 5.16 Comparison of measured travel time with calculated values

\begin{tabular}{|c|c|c|c|c|c|c|}
\hline \multirow{3}{*}{$\begin{array}{l}\text { Method of } \\
\text { Calculation }\end{array}$} & \multicolumn{3}{|c|}{$5 / 9 / 1999$} & \multicolumn{3}{|c|}{$6 / 20 / 1999$} \\
\hline & \multicolumn{2}{|c|}{$\begin{array}{c}\text { I-65 } \\
\text { (Major route) }\end{array}$} & \multirow{2}{*}{$\begin{array}{c}\begin{array}{c}\text { Alternate } \\
\text { route }\end{array} \\
\begin{array}{c}\text { Travel } \\
\text { Time }\end{array} \\
\end{array}$} & \multicolumn{2}{|c|}{$\begin{array}{c}\text { I-65 } \\
\text { (Major route) }\end{array}$} & \multirow{2}{*}{$\begin{array}{c}\begin{array}{c}\text { Alternate } \\
\text { route }\end{array} \\
\begin{array}{c}\text { Travel } \\
\text { Time }\end{array} \\
\end{array}$} \\
\hline & $\begin{array}{c}\text { Queueing } \\
\text { Delay } \\
\end{array}$ & $\begin{array}{c}\text { Travel } \\
\text { Time } \\
\end{array}$ & & $\begin{array}{c}\text { Queueing } \\
\text { Delay }\end{array}$ & $\begin{array}{c}\text { Travel } \\
\text { Time }\end{array}$ & \\
\hline $\begin{array}{l}\text { Field } \\
\text { Measurement }\end{array}$ & -- & 19.1 & \multirow{3}{*}{16.0} & -- & 20.35 & \multirow{3}{*}{22.43} \\
\hline $\begin{array}{l}\text { Calculated values } \\
\text { using hour-by- } \\
\text { hour volumes }\end{array}$ & 11.04 & 17.9 & & 9.39 & 20.19 & \\
\hline $\begin{array}{l}\text { Percentage of } \\
\text { difference }\end{array}$ & -- & $6.3 \%$ & & -- & $0.8 \%$ & \\
\hline
\end{tabular}




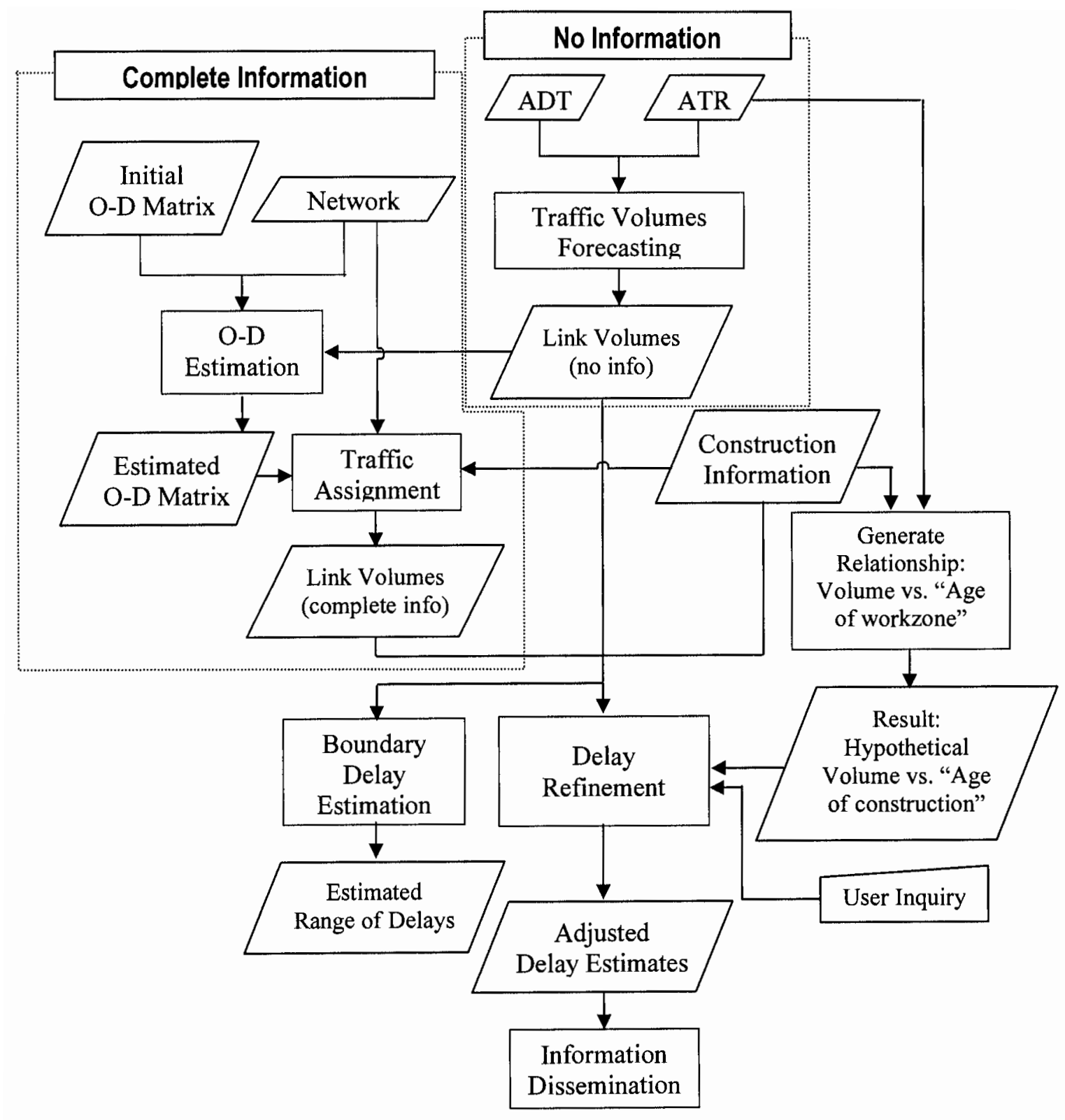

Figure 5.1 Research framework 


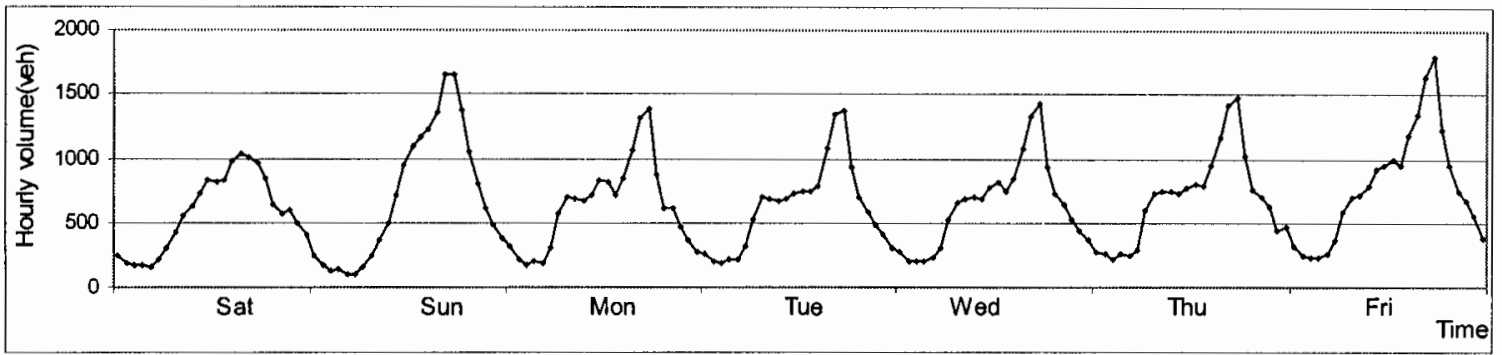

Figure 5.2(a) Northbound Traffic Patterns for Normal Days at Station 2400

(November 1997)

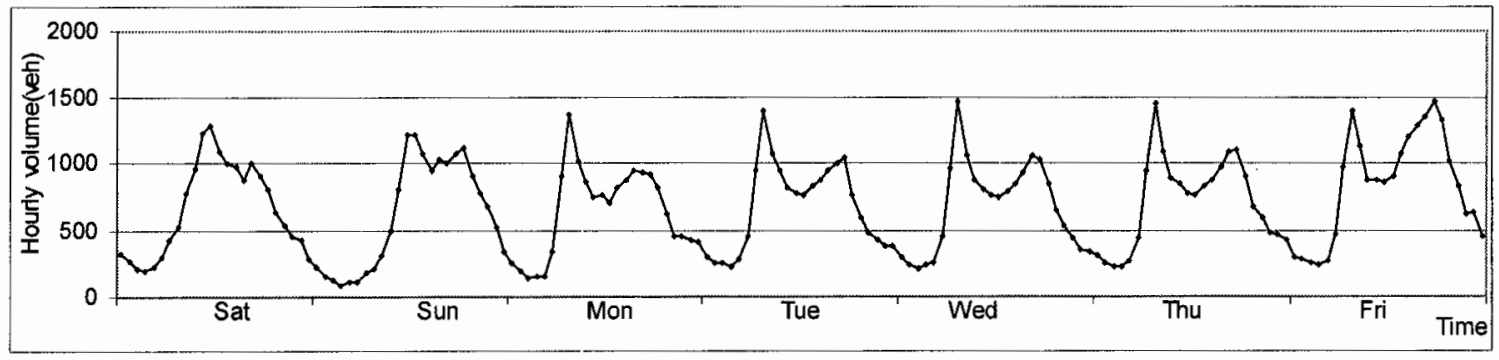

Figure 5.2(b) Southbound Traffic Patterns for Normal Days at Station 2400 (November 1997) 
Hourly Volumes (vph)
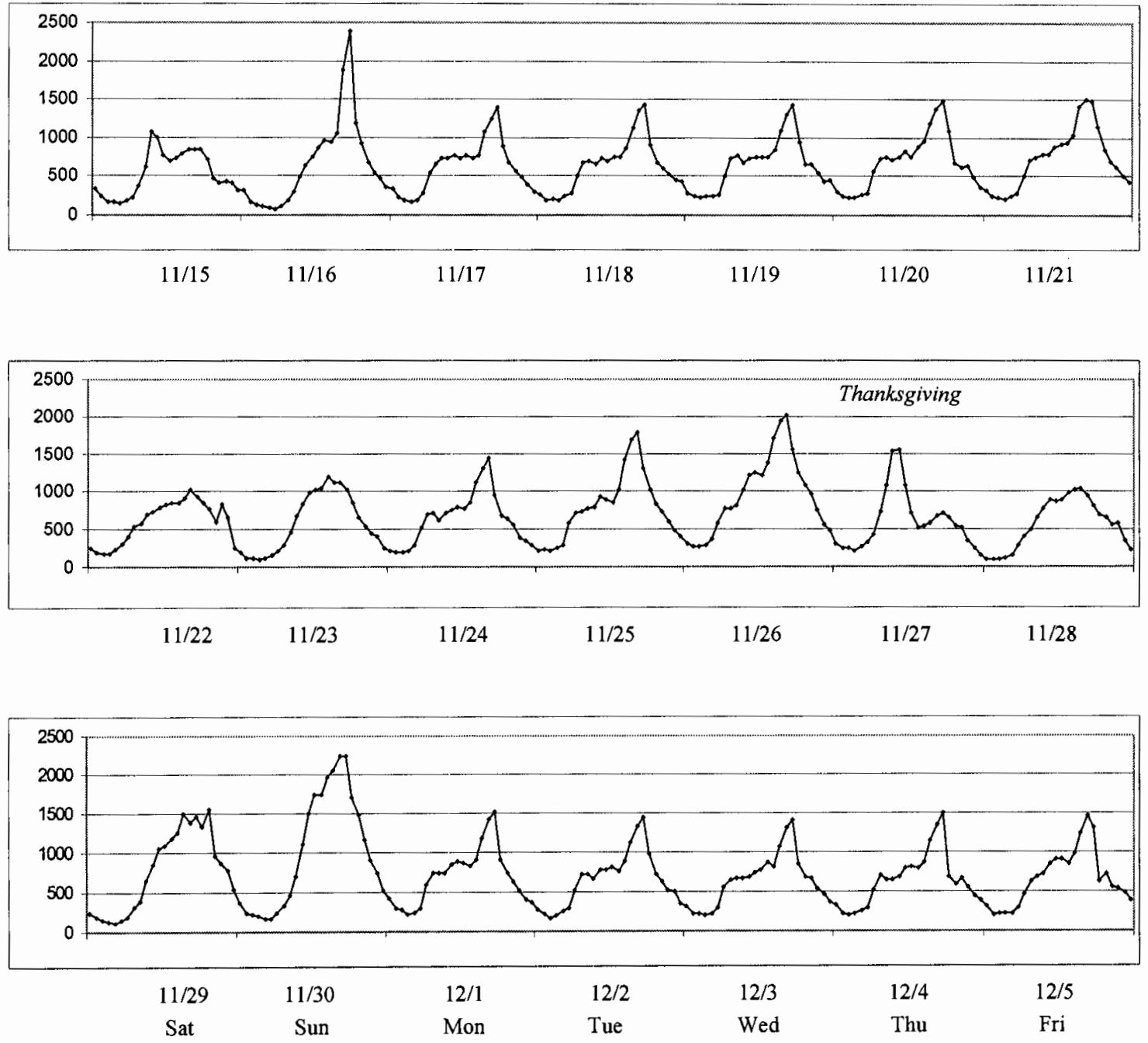

Figure 5.3(a) Northbound Traffic Patterns for the weeks before, during, and after Thanksgiving Holiday at Station 2400 (November 1997) 
Hourly Volumes (vph)
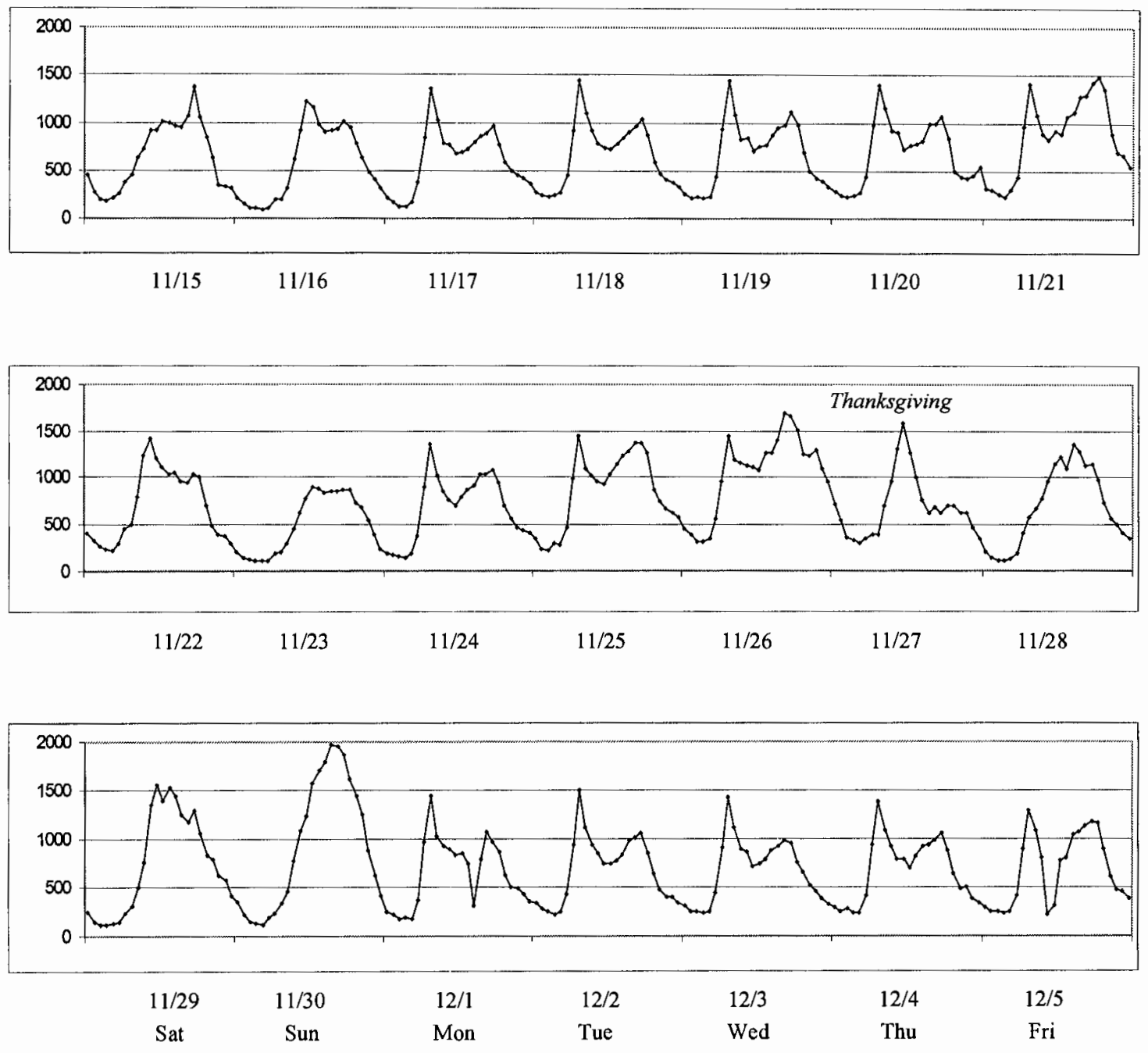

Figure 5.3(b) Southbound Traffic Patterns for the weeks before, during, and after Thanksgiving Holiday at Station 2400 (November 1997) 
Hourly Volumes (vph)
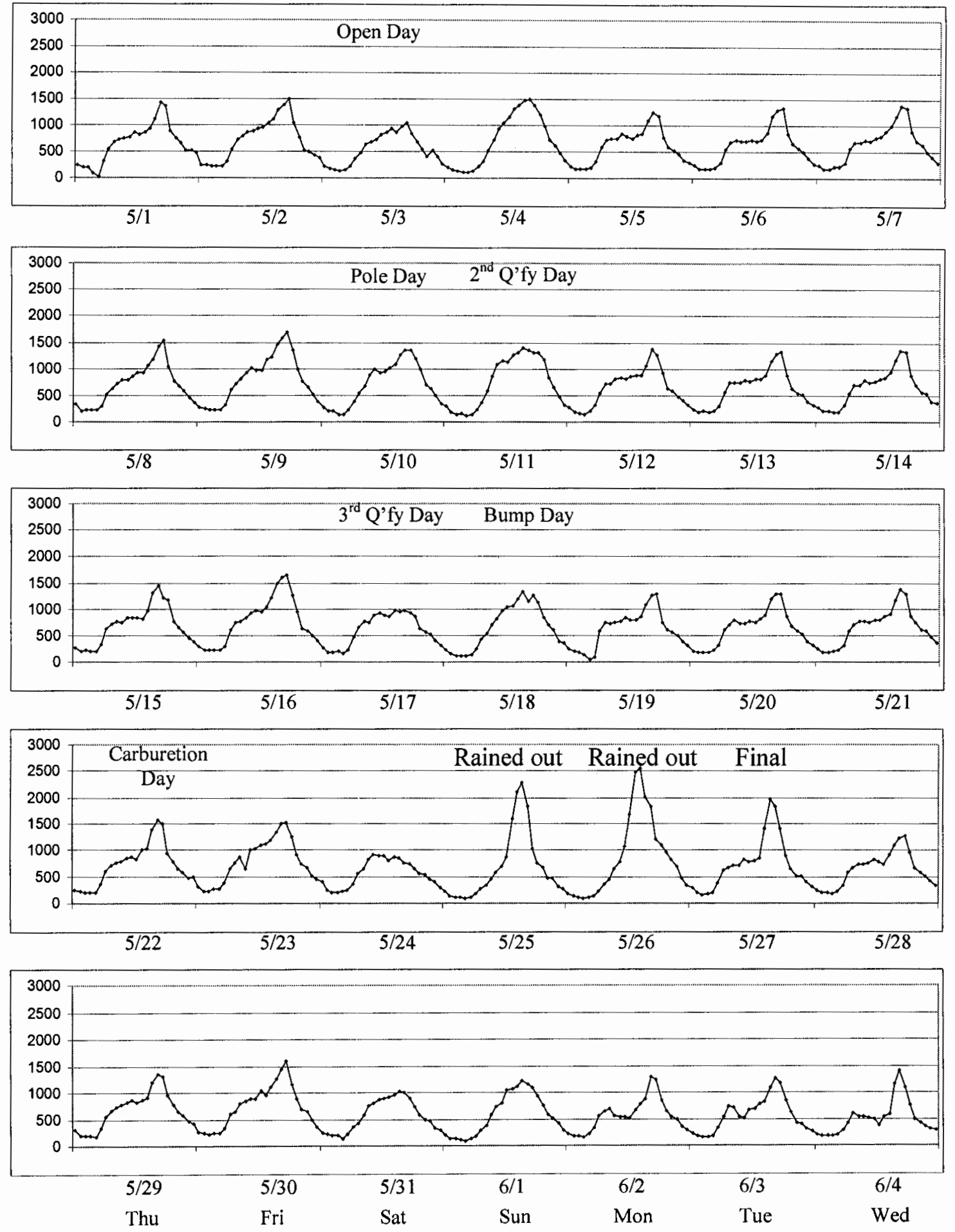

Figure 5.4 Northbound Traffic Patterns for Indy500 Car Racing Event at Station 2400 (May 1997) 


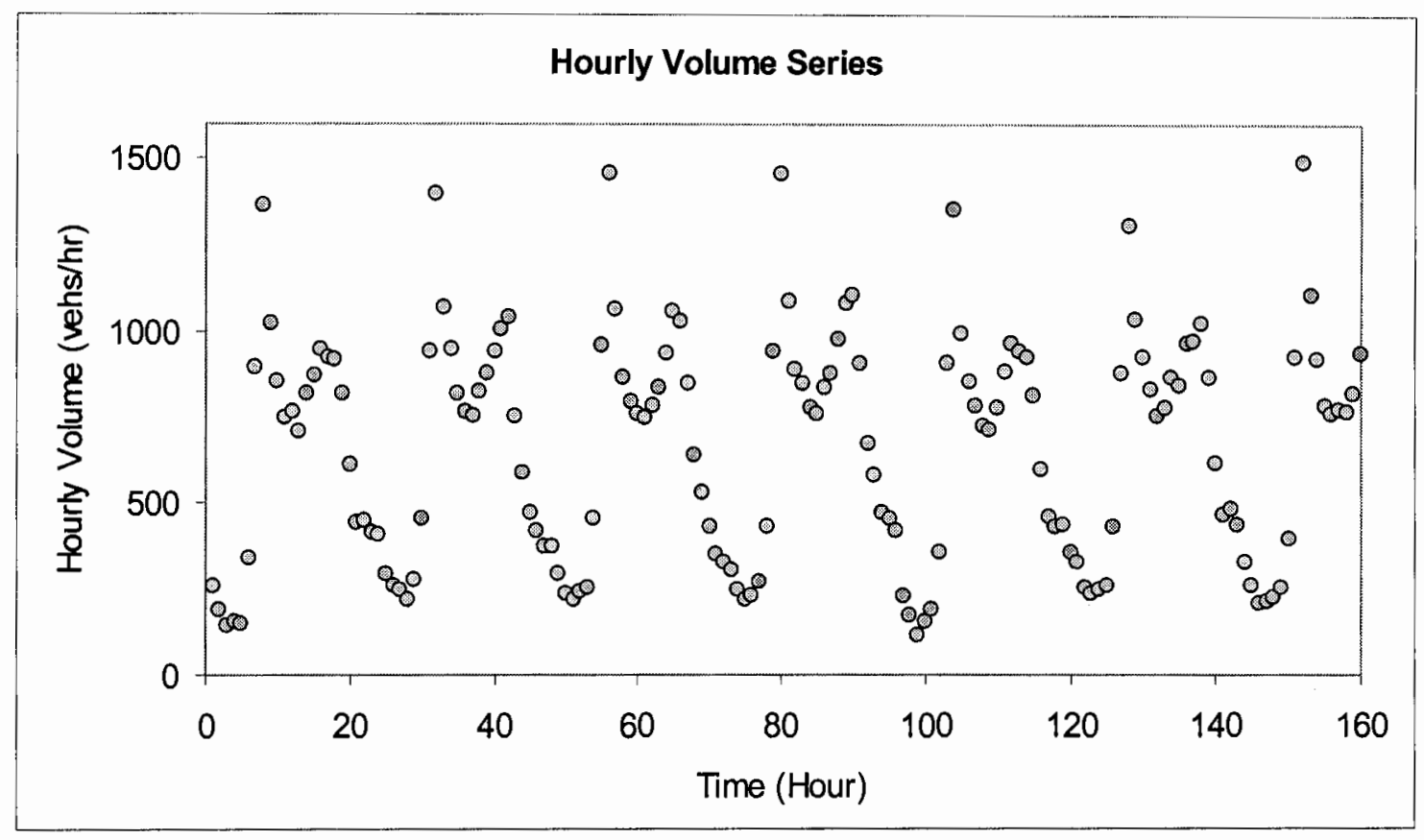

Figure 5.5 The first 160 weekday hourly volumes at ATR station 2400 in November 1997 


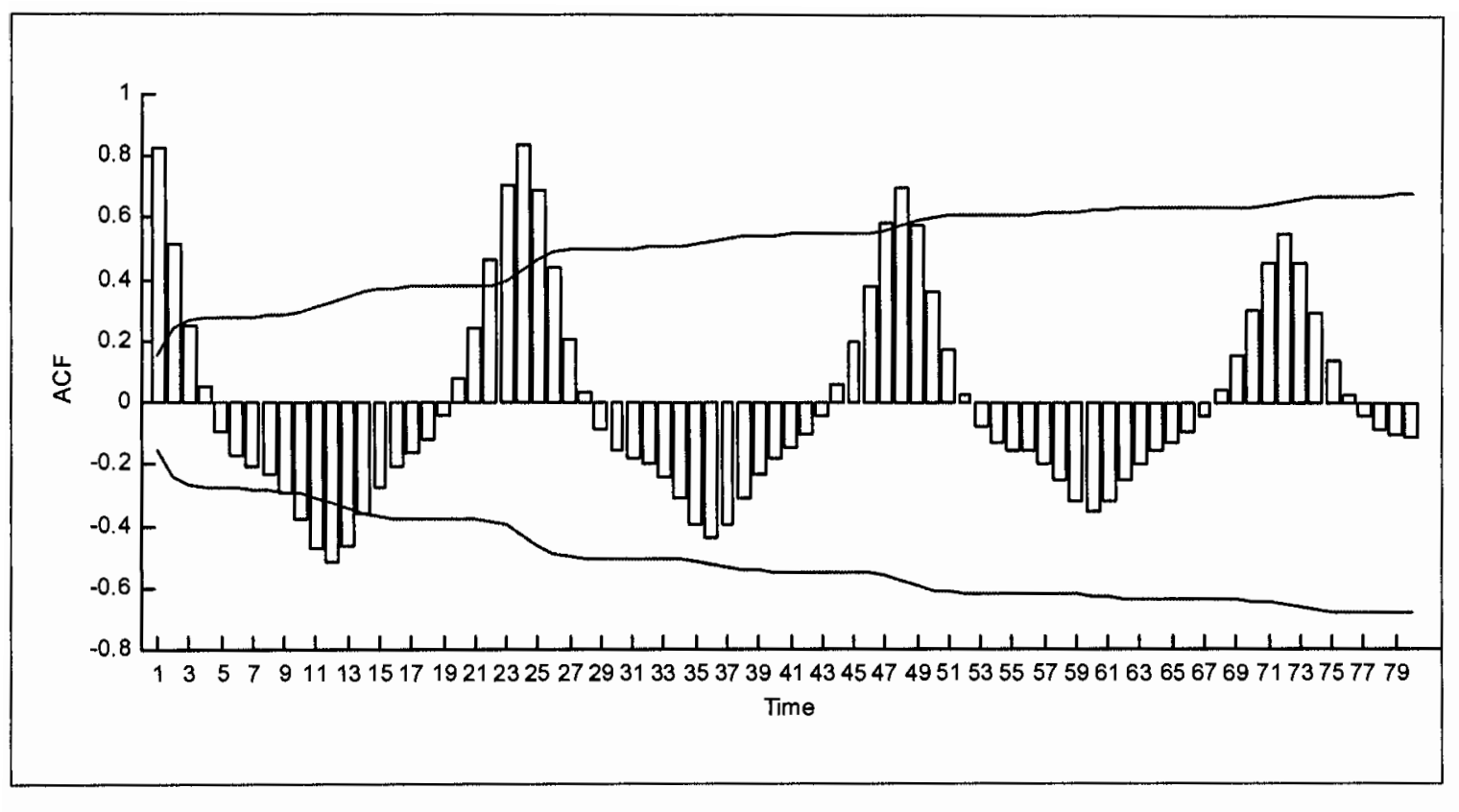

Figure 5.6 Autocorrelation functions of the original series 


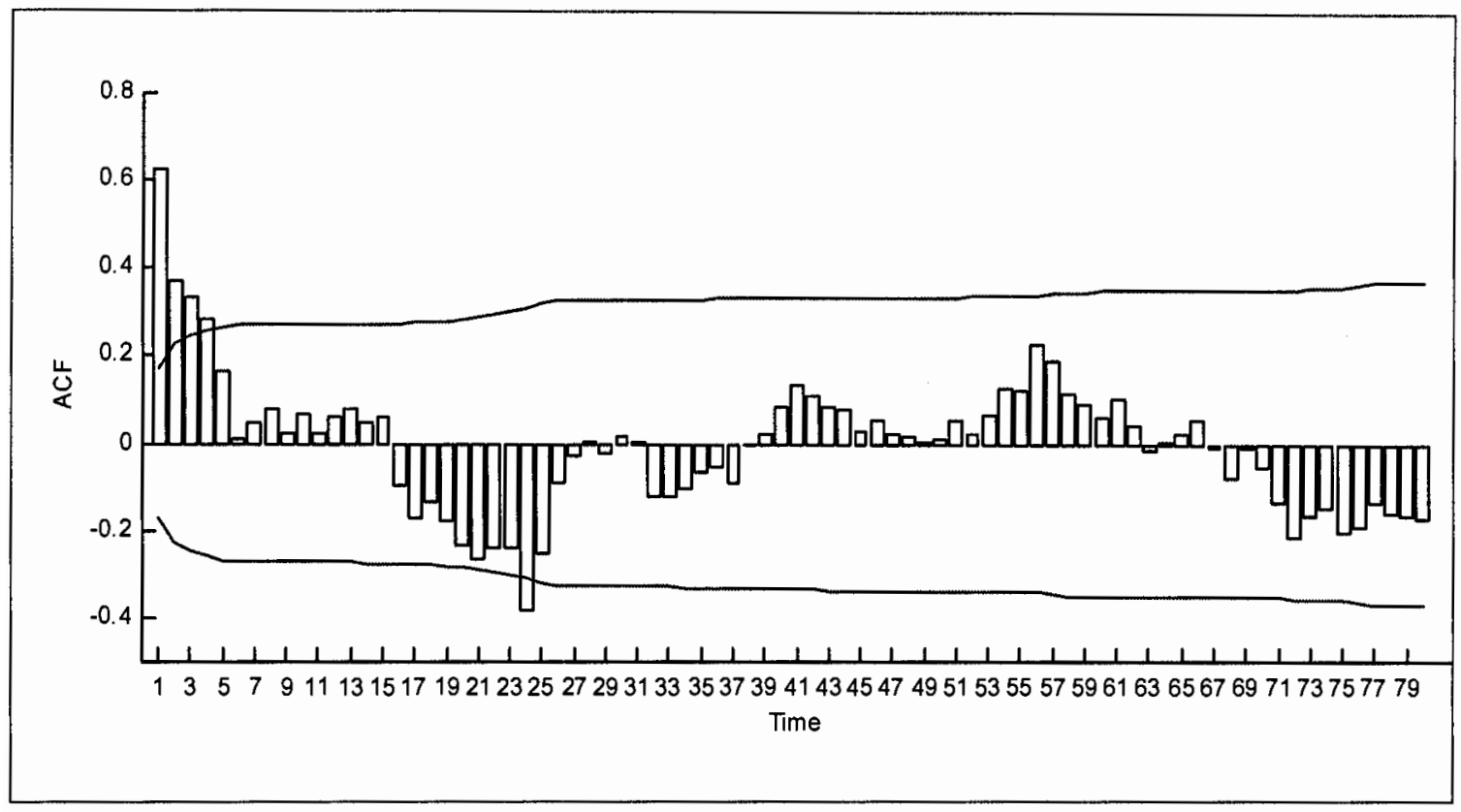

Figure 5.7 Autocorrelation functions of $\left(1-B^{24}\right) Z_{t}$ 


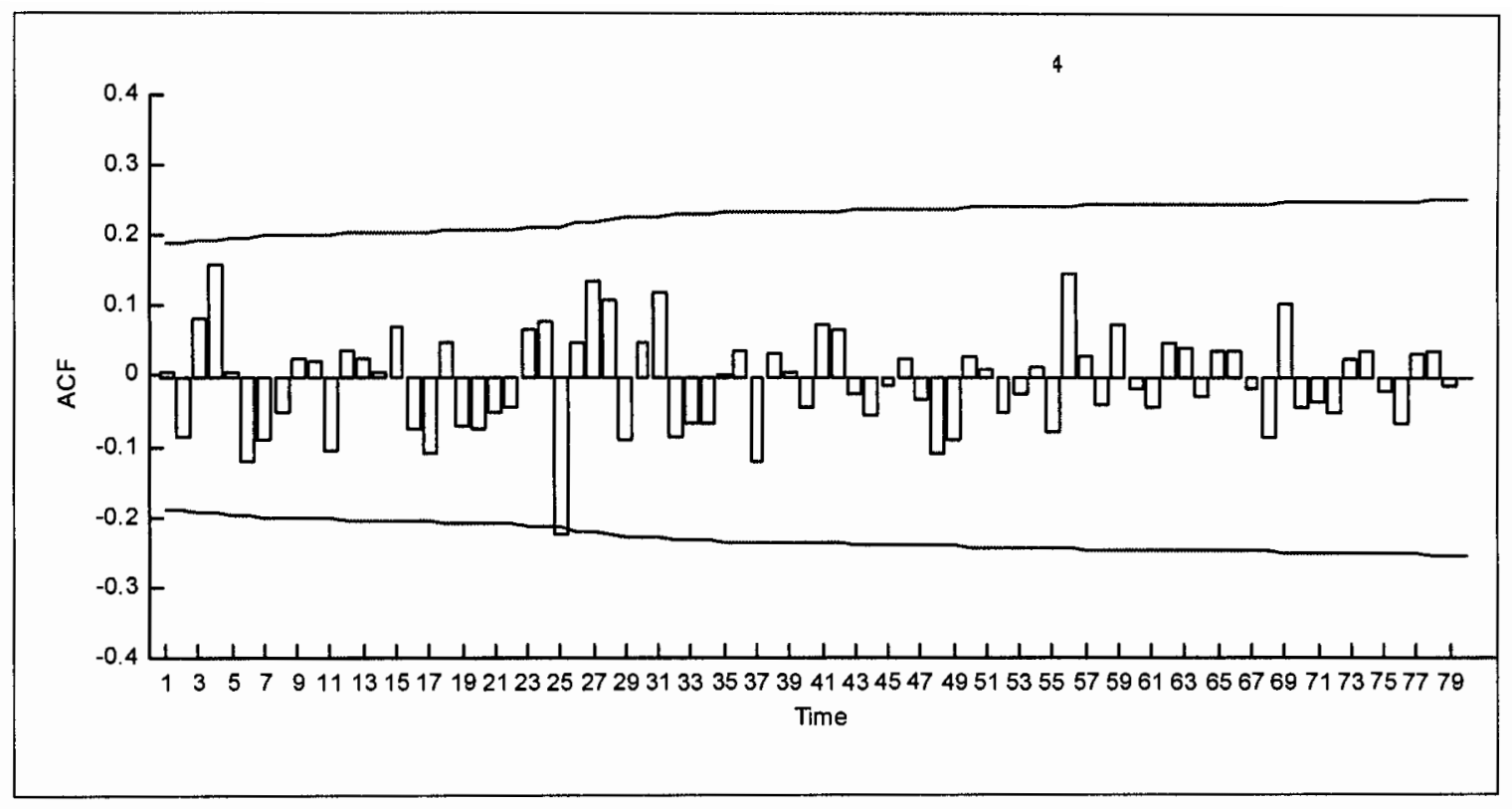

Figure 5.8 ACFs of the residuals of model $(1,0,0)(1,1,1)_{24}$ 


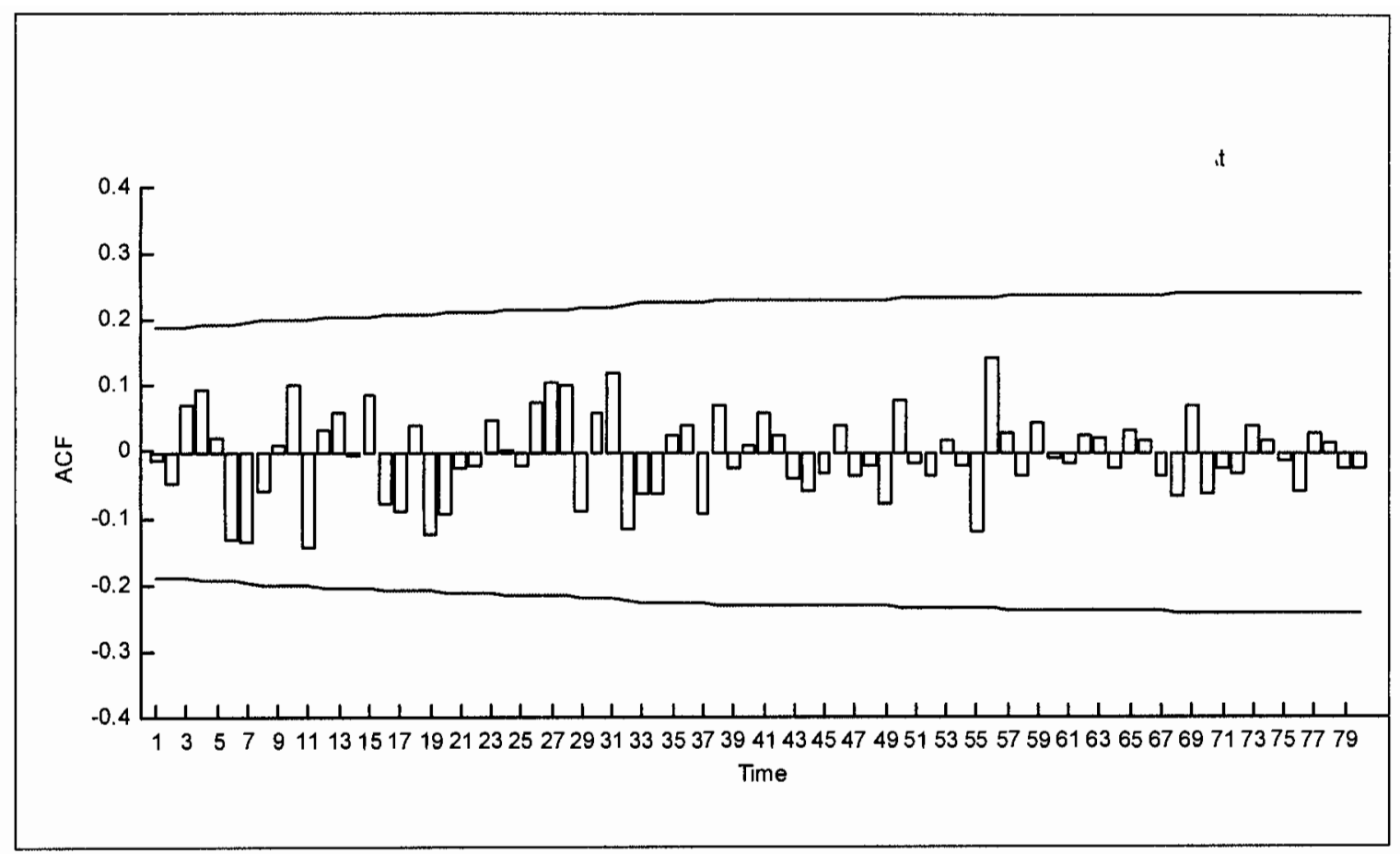

Figure 5.9 ACFs of the residuals of model $\left(1,0,1_{25}\right)(1,1,2)_{24}$ 


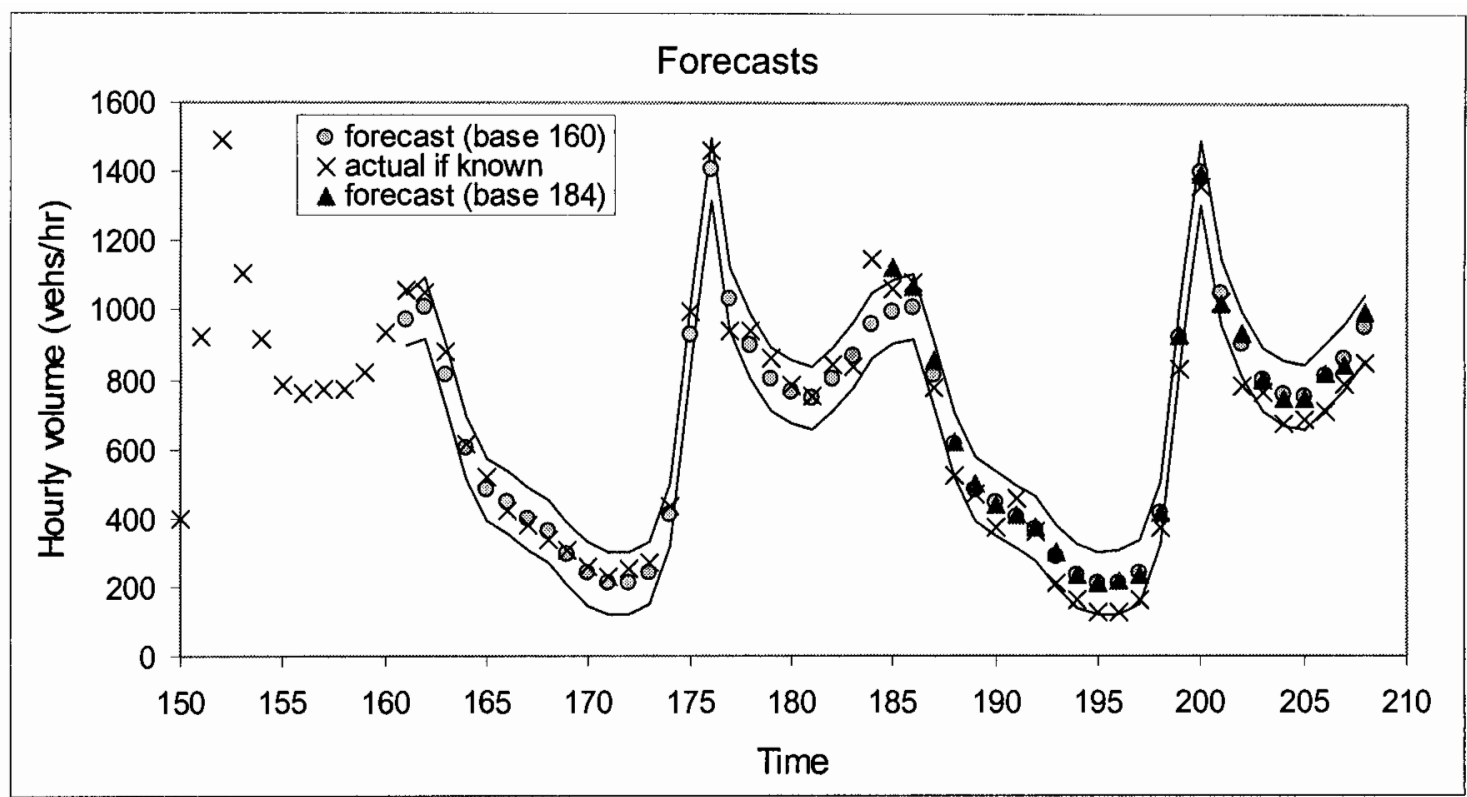

(note) The lines in the figure show 95\% upper and lower confidence limits.

Figure 5.10 48-period-ahead forecasts of hourly traffic volumes at base period 160 and 24-periods-ahead updated forecasts of hourly traffic volumes at base period 184 (November 1997) 


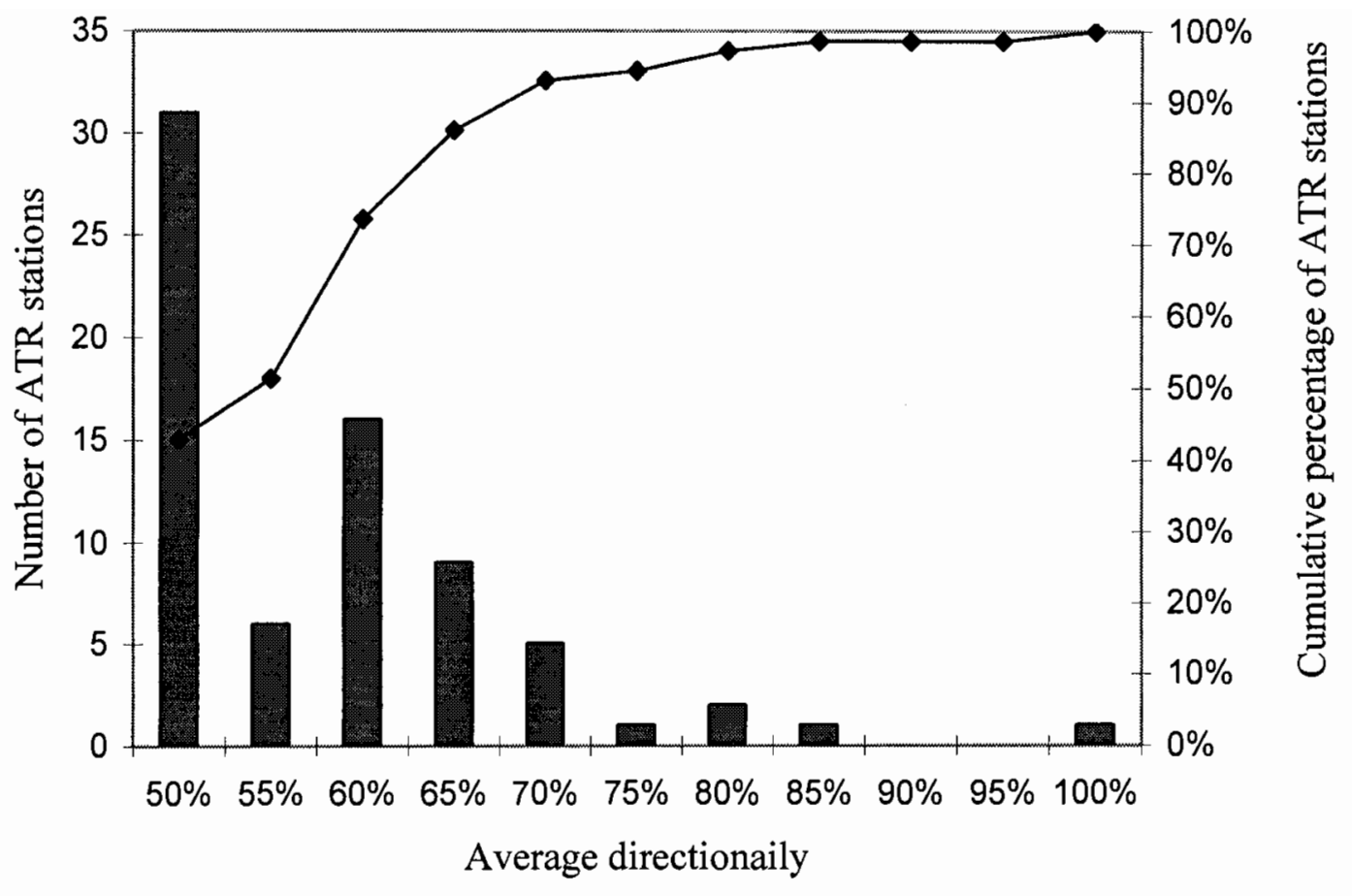

Figure 5.11 Definition of bad data of high directionality 


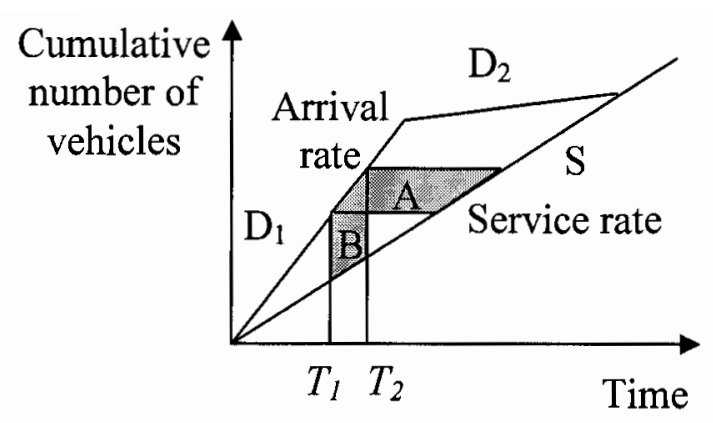

Area A: total queueing delays of vehicles arriving the system between $T_{1}$ and $T_{2}$

Area B: total queueing delays of vehicles in the system between $T_{1}$ and $T_{2}$

Figure 5.12 A typical queueing diagram 


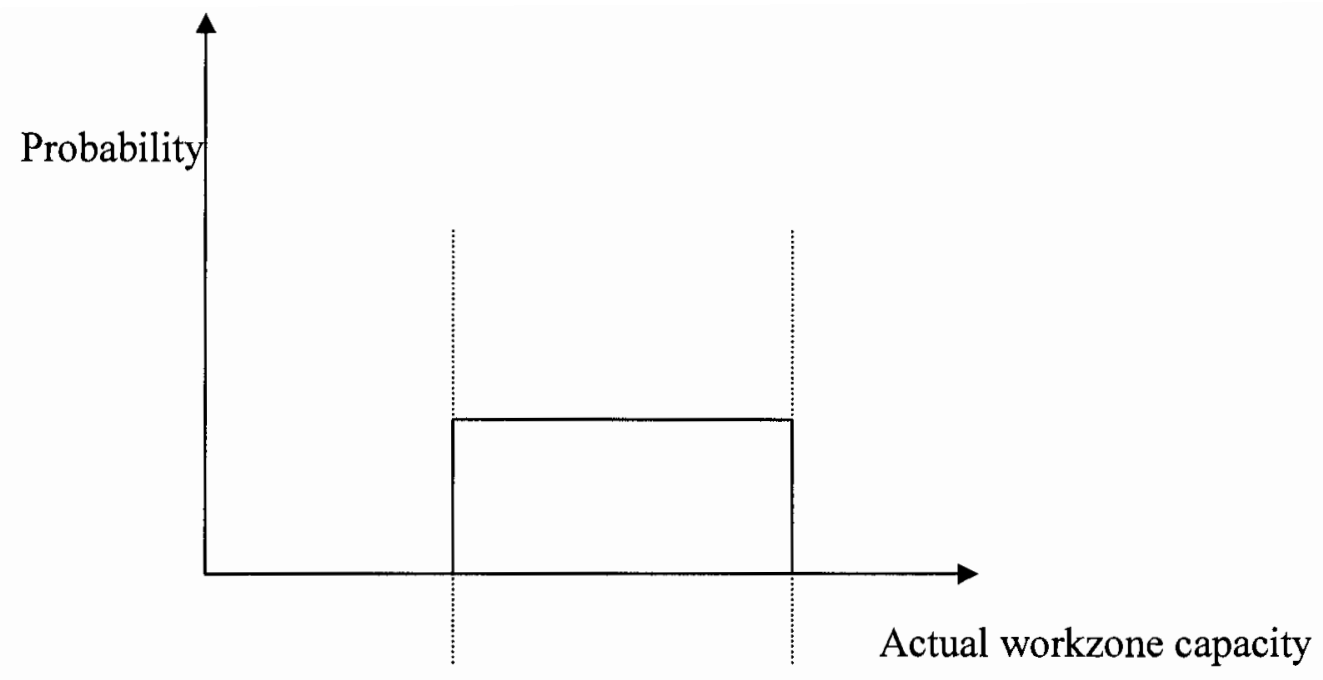

$\begin{array}{cc}\begin{array}{c}\text { Estimated } \\ \text { capacity when }\end{array} & \begin{array}{c}\text { Estimated } \\ \text { capacity when } \\ \text { CERF }=100\end{array} \\ \text { CERF }=0\end{array}$

Figure 5.13 Probability distribution of actual workzone capacity 


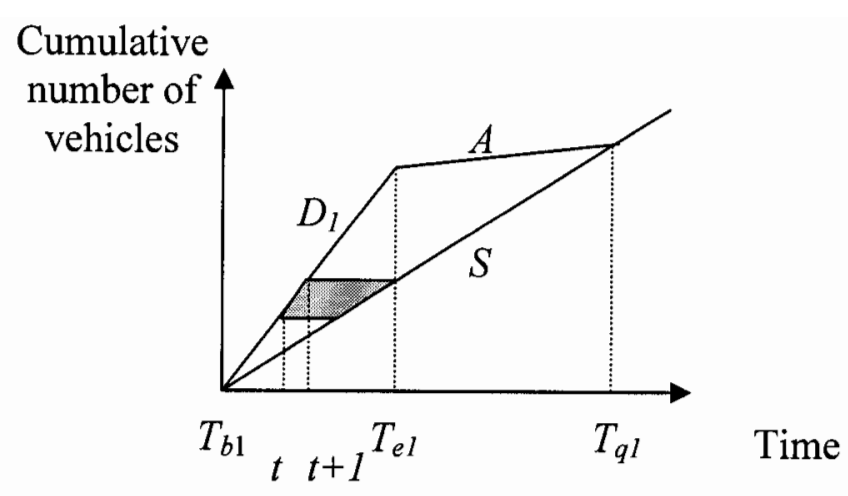

AM-peak periods

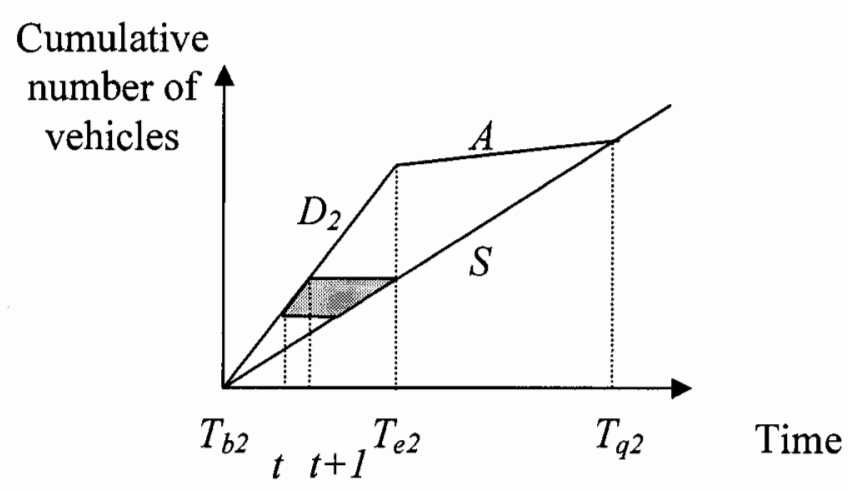

PM-peak periods

Figure 5.14 A queueing diagram for situations when the AM-peak period is not followed immediately by the PM-peak period $\left(T_{b l} \leq t<T_{e l}\right)$ 


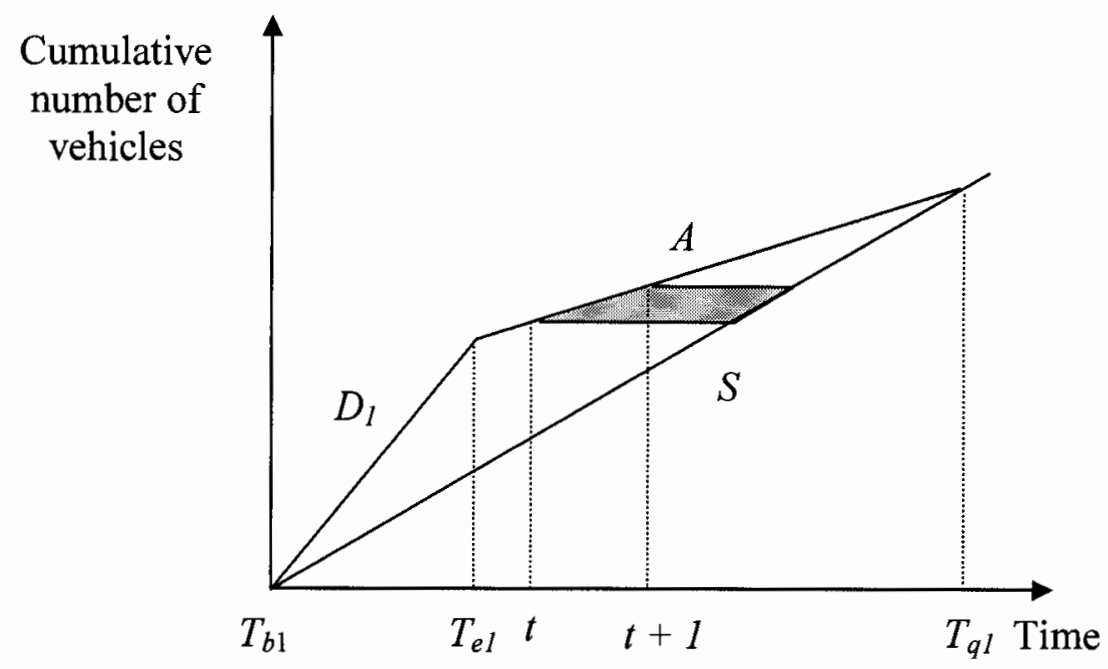

Figure 5.15 A queueing diagram for situations when the AM-peak period is not followed immediately by the PM-peak period $\left(T_{e l} \leq t<T_{q l}\right.$ and $\left.t+l<T_{q l}\right)$ 


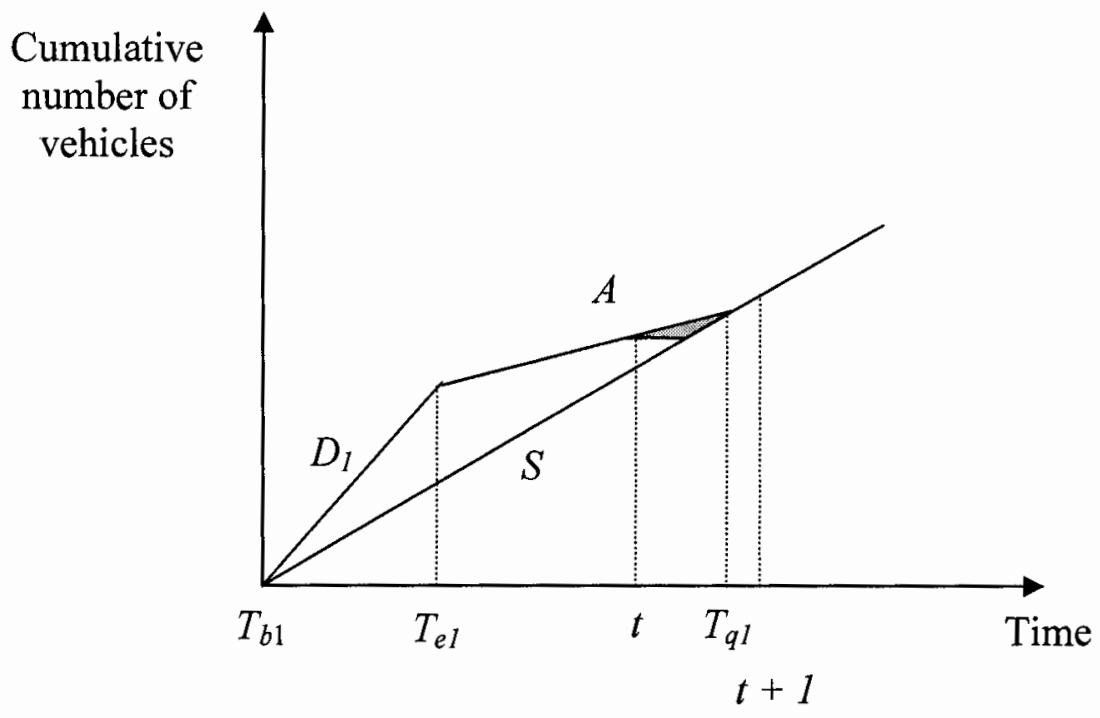

Figure 5.16 A queueing diagram for situations when the AM-peak period is not followed immediately by the PM-peak period $\left(T_{e l} \leq t<T_{q 1}\right.$ and $\left.t+1 \geq T_{q 1}\right)$ 


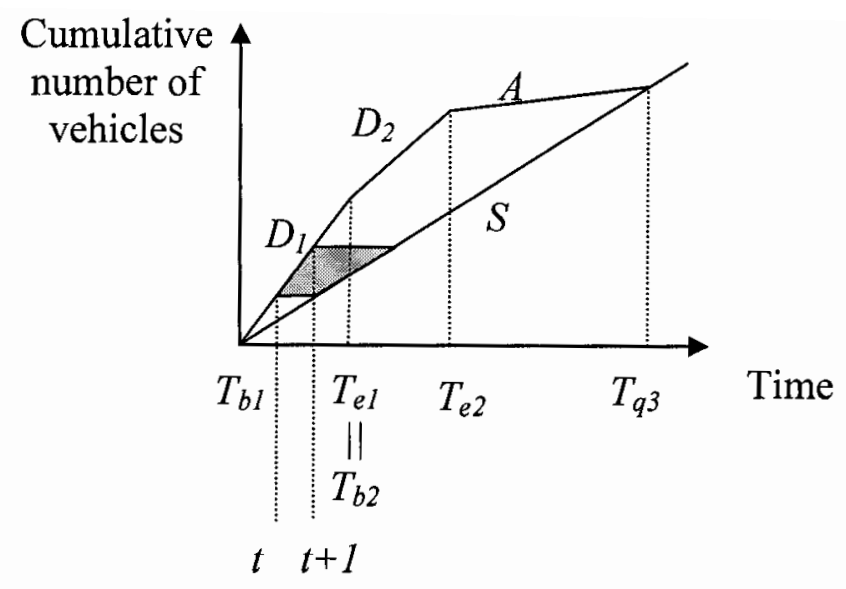

For situations when the queue does not dissipate completely until after the PM-peak period ends

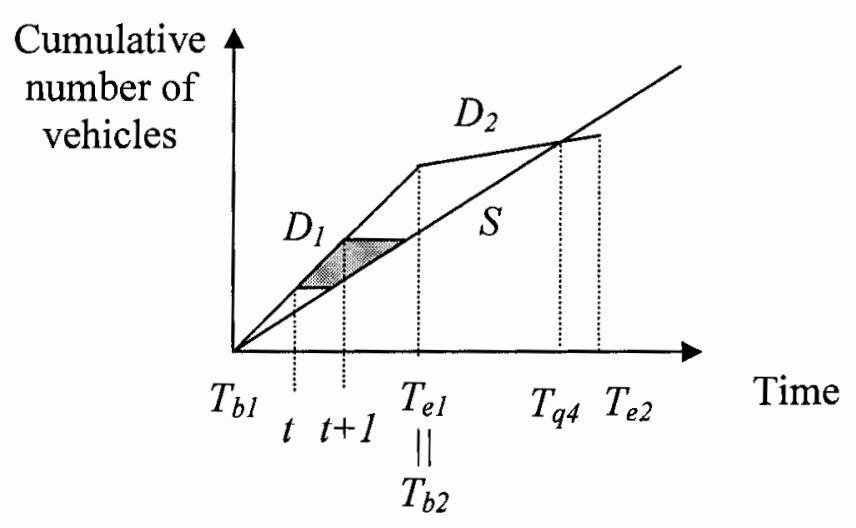

For situations when the queue dissipates completely after the AM-peak period ends but before the PM-peak periods ends

Figure 5.17 Queueing diagrams for situations when the PM-peak period comes immediately after the AM-peak period 


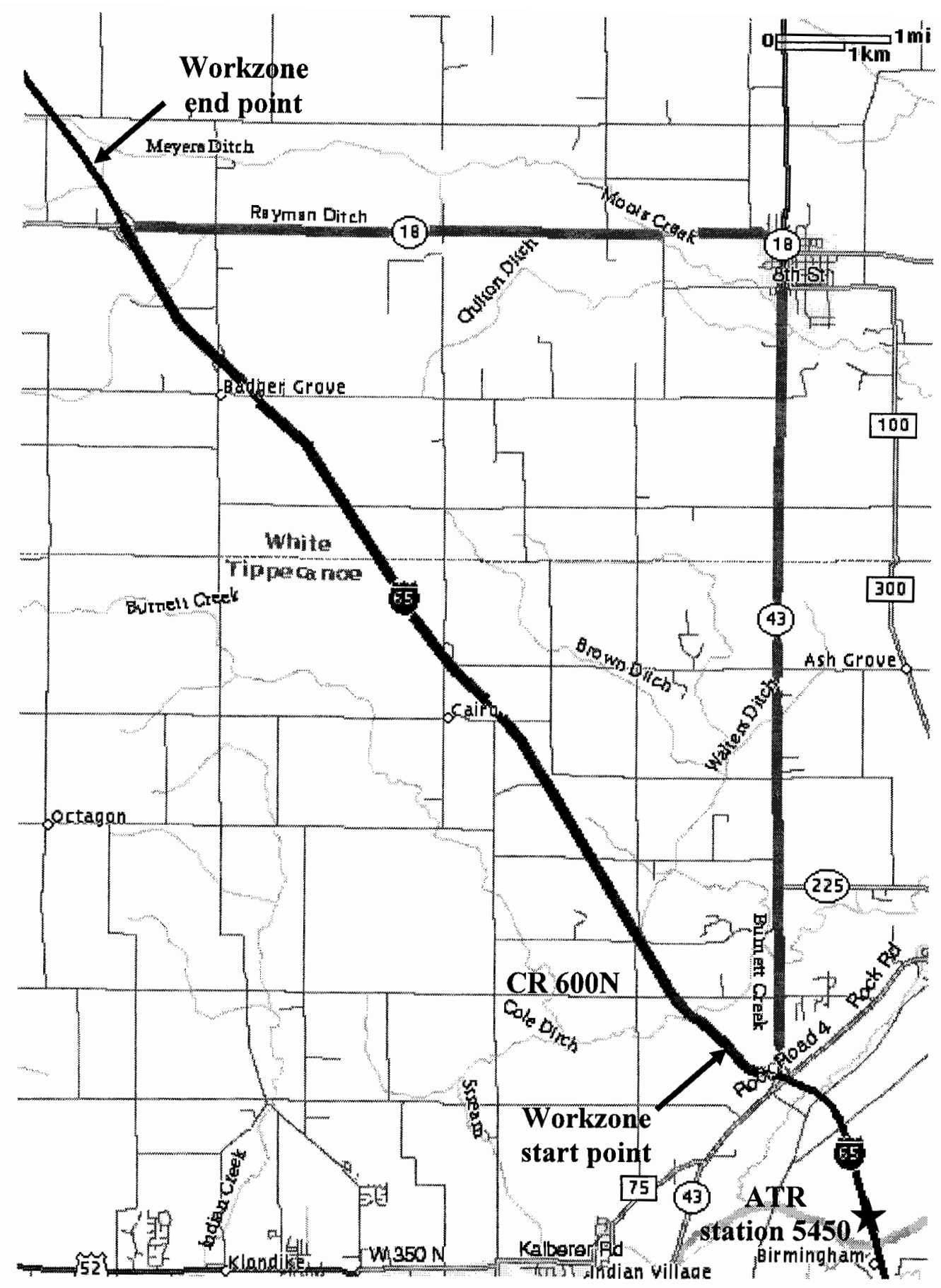

Major route

Alternate route

Figure 5.18 Field map of traffic survey on 5/9/99 


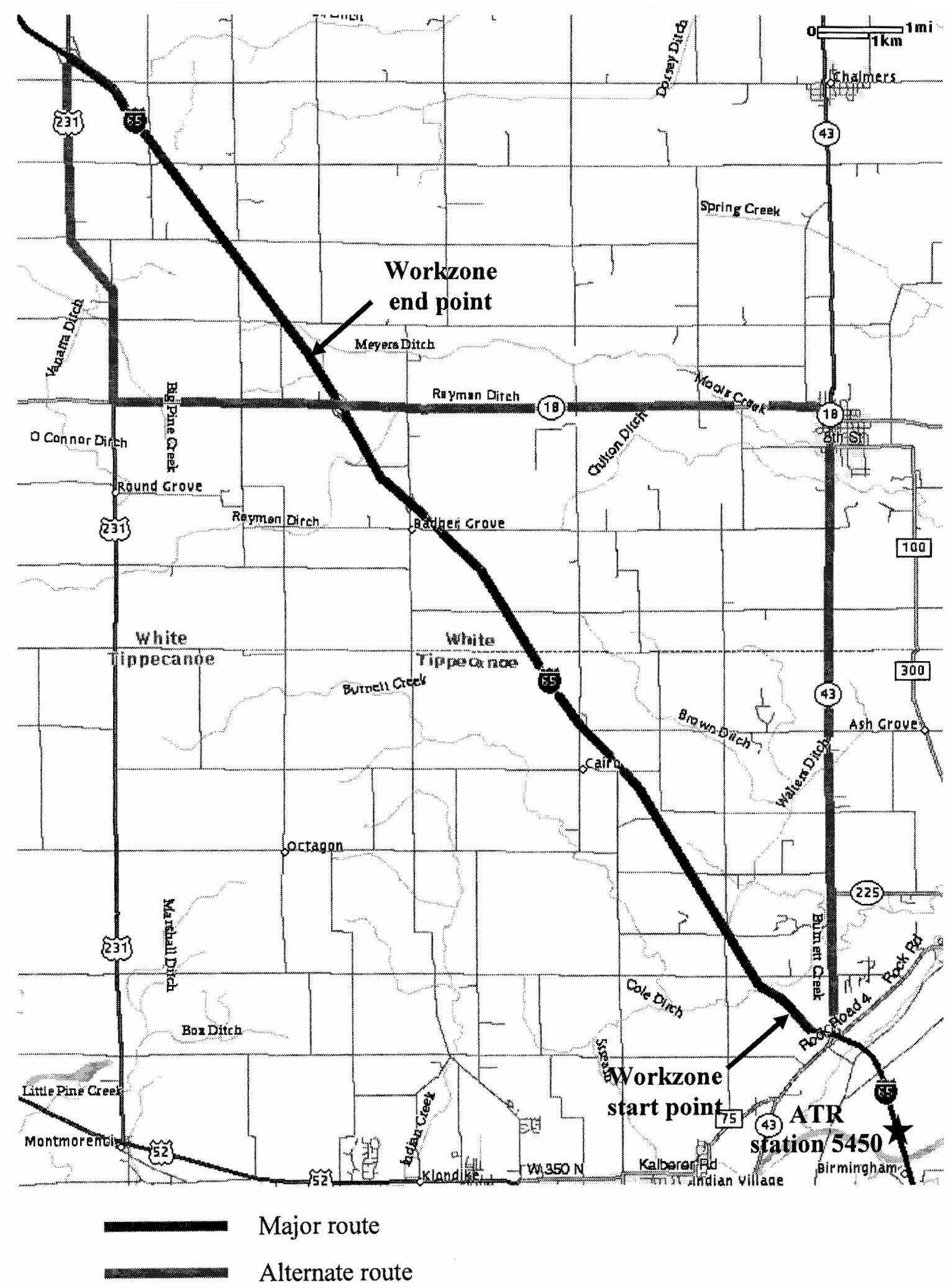

Figure 5.19 Field map of traffic survey on 6/20/99 


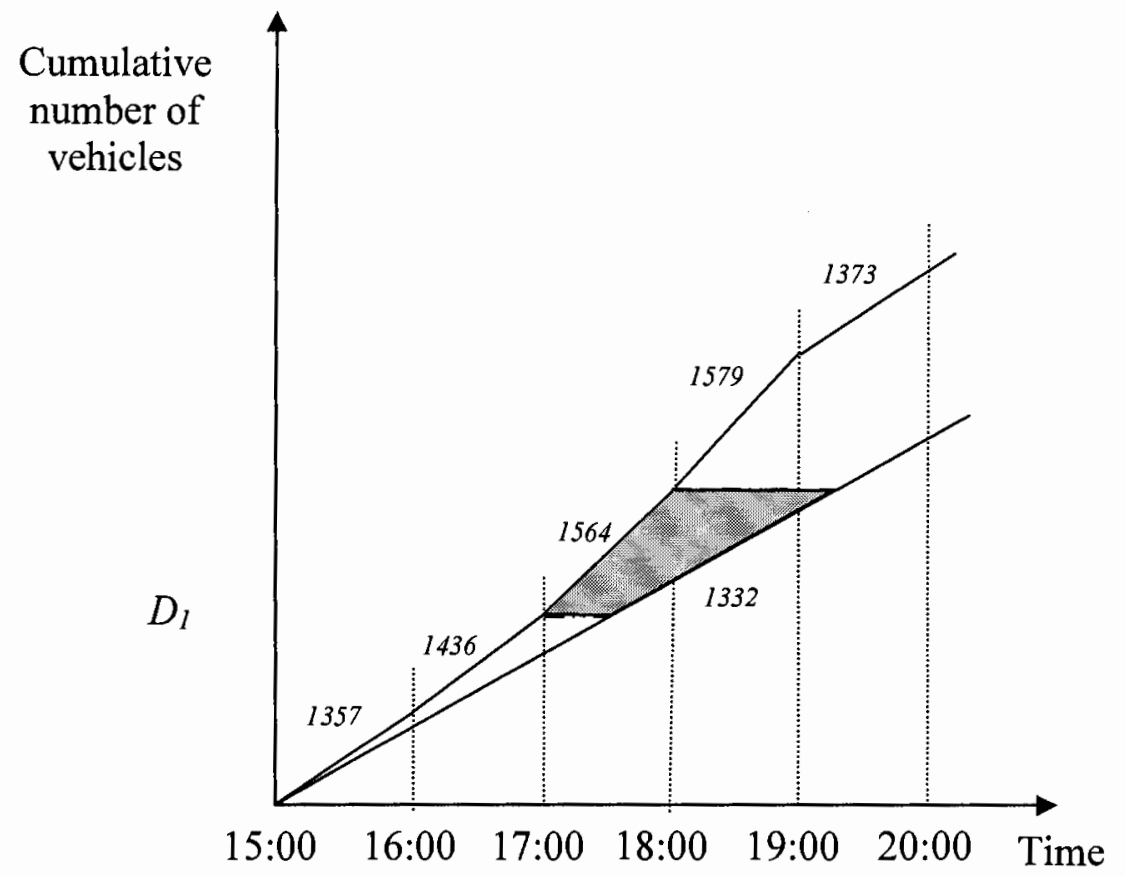

Figure 5.20 Queueing diagram for the first survey*

* Graph is not drawn to scale. 


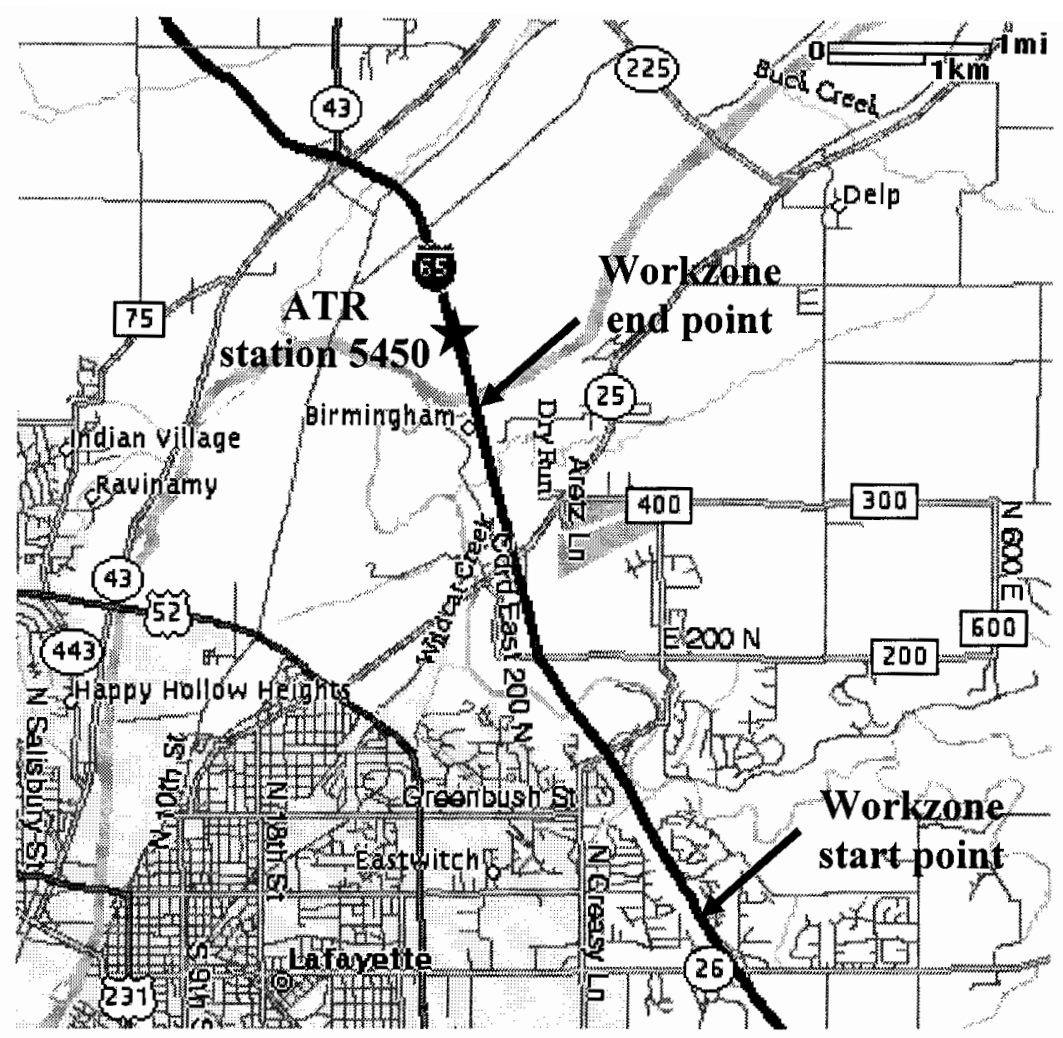

Figure 5.21 Field map of traffic survey on 4/26/2000 through 5/9/2000 


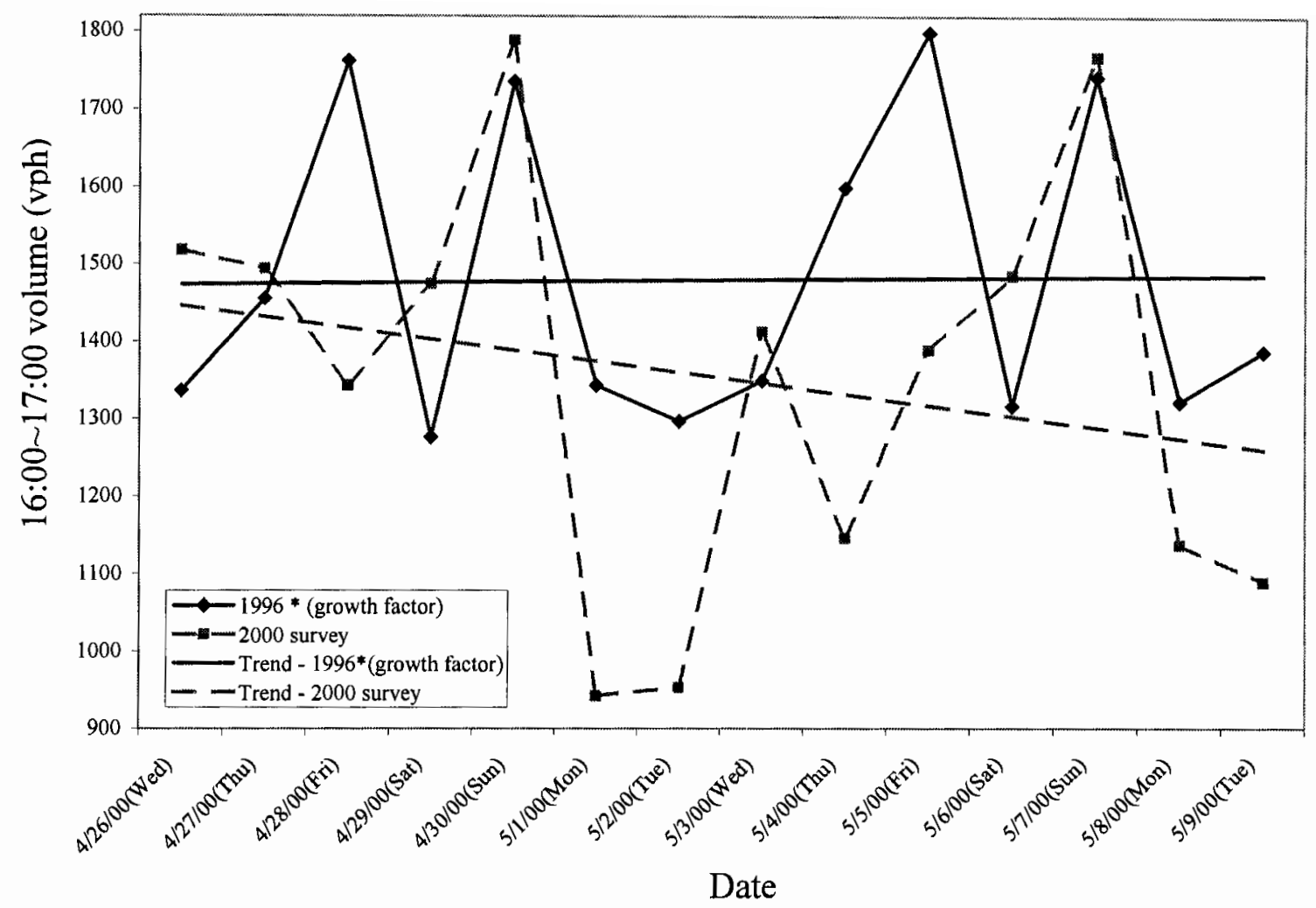

Figure 5.22 Comparison of the demands from survey in 2000 and the historical volumes in 1996 


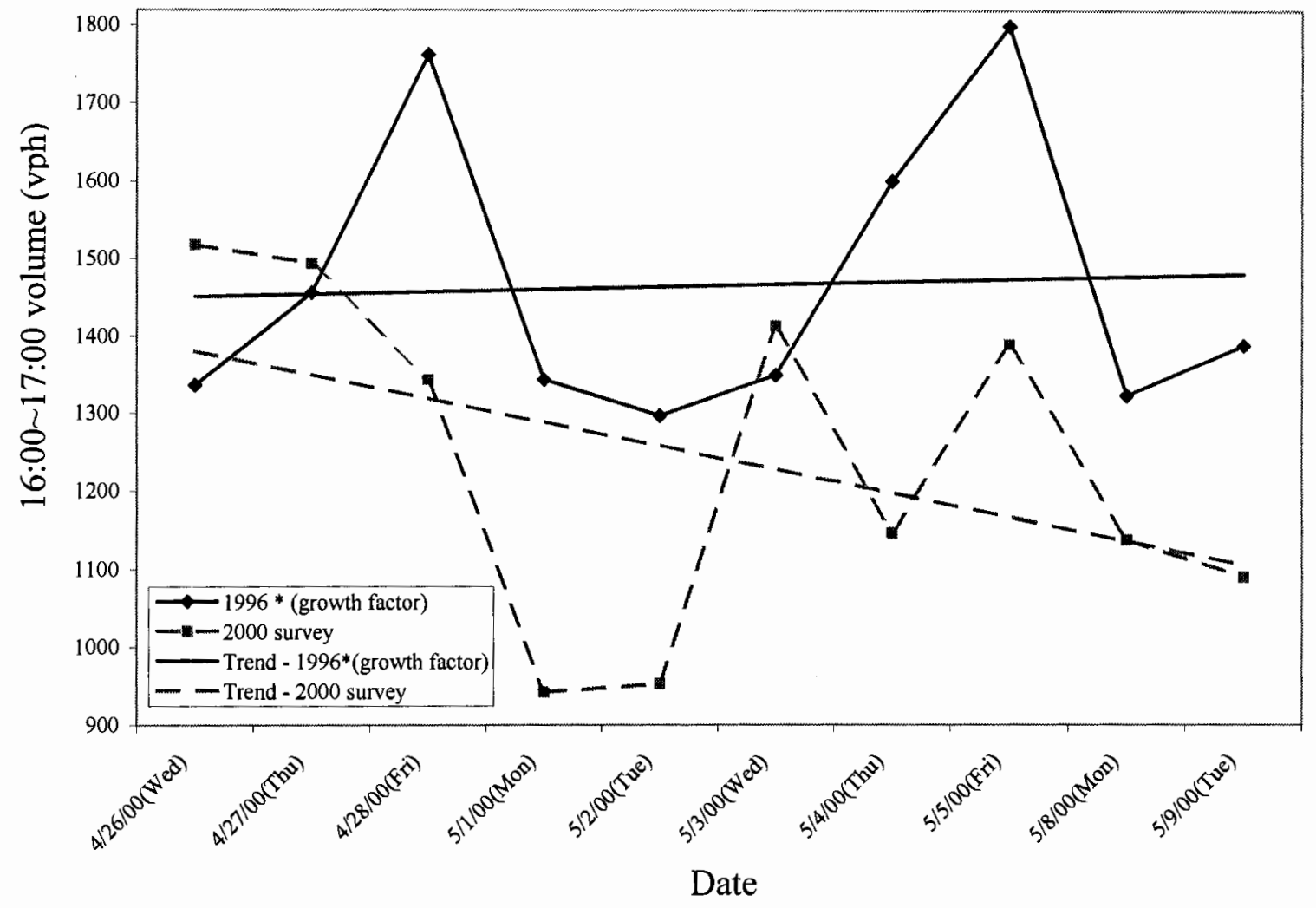

Figure 5.23 Comparison of the demands from survey in 2000 and the historical volumes in 1996 (excluding weekedns) 


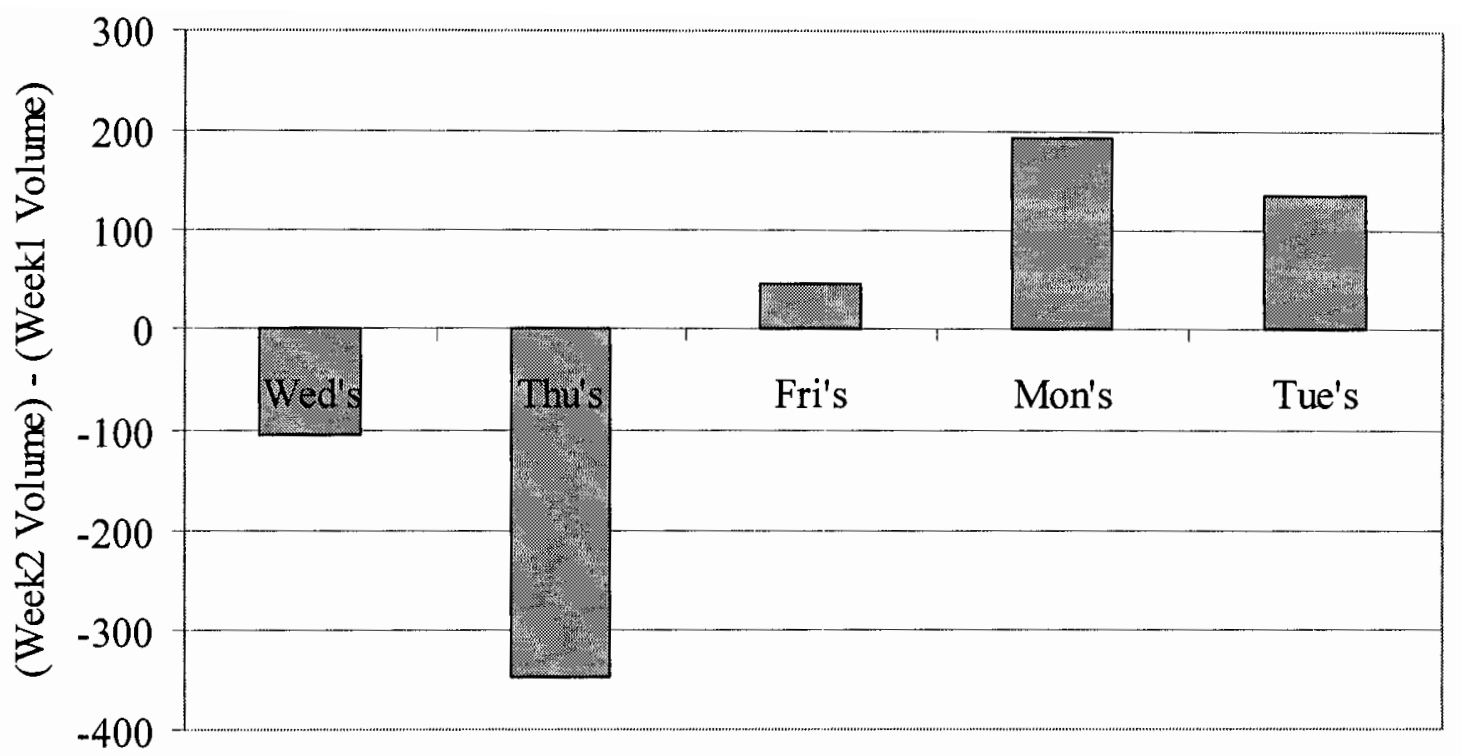

Figure 5.24 Difference between the demands from survey on the second week and on the first week 


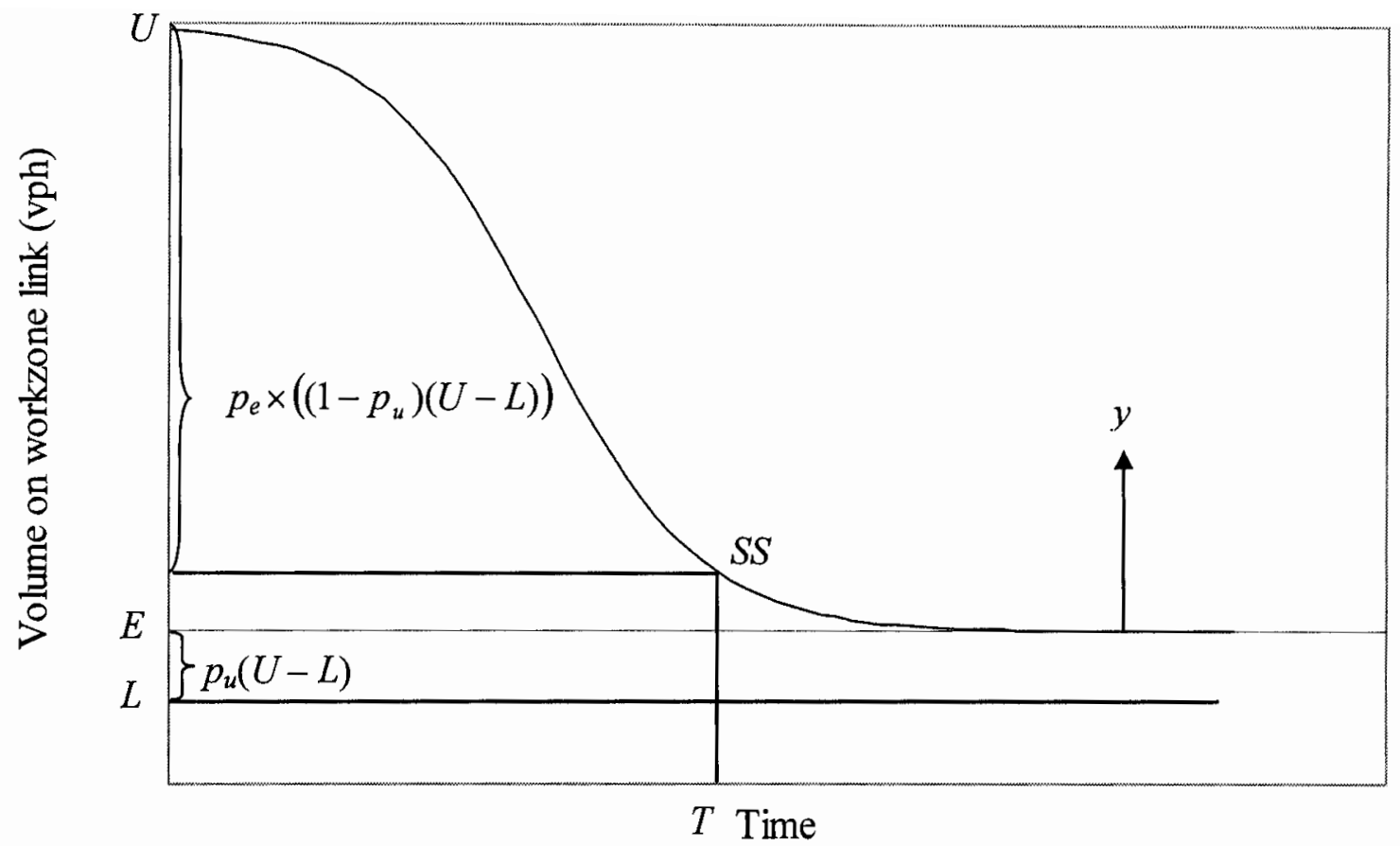

Figure 5.25 A hypothesized relationship between the volume on workzone link and the age of construction using a logistic function 


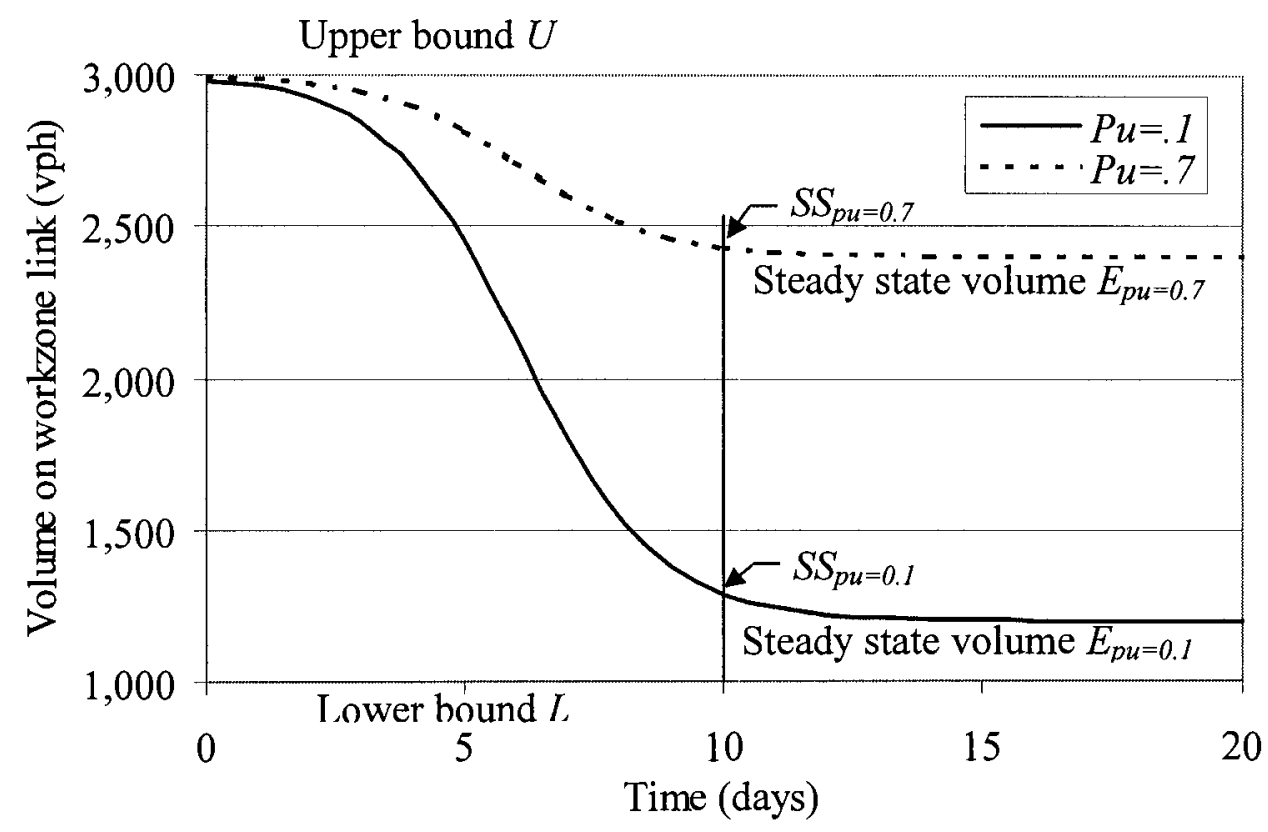

Figure 5.26 Logistic curves for two different percentages of uninformed travelers 


\section{SYSTEM DEVELOPMENT}

The functions of this Highway Travel Information System (HTIS) are not only to provide INDOT a system to maintain travel information and assist project scheduling, but also to provide road users a means to plan their trips in advance. The personnel who are in charge of maintaining travel information system need to build and update travel information with a minimum of effort. The personnel who are in charge of project scheduling need to determine a scheduling strategy that reduces total delays with reasonable construction costs. These needs are different from those of travelers who would like to do pre-trip planning. Because of these different needs, different travel information subsystems are necessary. Therefore, the HTIS is divided into three subsystems: modeling, project scheduling and web access.

The modeling and project scheduling subsystems are regarding system maintenance. The major function of modeling subsystem is to predict traffic conditions and produce travel information. The function of the project scheduling subsystem is to maintain and review road construction information. These two subsystems are dependent on each other. The modeling subsystem requires information on initial construction schedules from the project scheduling subsystem to allow the evaluation of traffic conditions in workzones. In turn, the project scheduling subsystem requires inputs on the traffic conditions at workzones from the modeling subsystem, to allow scheduling adjustments. 
The iterations between these two subsystems end when both the project schedule and its effects on traffic are acceptable.

The web access subsystem is for use of highway travelers. The function of the web access subsystem is to provide drivers with predicted traffic conditions based on their departure time. Highway users may perform pre-trip planning to minimize possible delays by repeatedly querying the web access subsystem for the traffic conditions provided by the modeling subsystem.

The relationship of the three subsystems is shown in Figure 6.1.

The HTIS is based on multiple software packages, because of the need for accessibility by the three groups of users and the capabilities of each software package. The tasks required for this system include traffic volume forecasting, O-D estimation, traffic assignment, delay estimation, road construction scheduling, and web publishing. Although these functions can be incorporated in TransCAD using its built-in macro language, GIS Developer's Kit (GISDK), the system built solely on TransCAD will be limited to only those who have access to TransCAD. In considering access by most users, some parts of the system that can be separated from the main system. Those parts with limited inputs and outputs $(\mathrm{I} / \mathrm{O})$ and that do not require the support of TransCAD are built with software packages that are more accessible, such as Microsoft Excel and Microsoft Access.

The remaining part of this chapter is organized as follows. Section 6.1 explained the development of the modeling subsystem, Section 6.2 the development of the project scheduling subsystem, Section 6.3 the development of the web access subsystem, and 
Section 6.4 the issues related to system maintenance. For more information regarding the use of each subsystem, please refer to Appendix A.

\subsection{Modeling Subsystem}

The function of the modeling subsystem is to predict traffic conditions and to produce travel information. This subsystem involves most of the analysis procedures including traffic volume forecasting, O-D estimation, traffic assignment, and delay estimation.

Among the procedures required in the modeling subsystem, the traffic volume forecasting meets the criterion of being separated from the main system, which includes limited I/O (input of ATR file and output of peak period factors) and not requiring the use of 'TransCAD. Therefore, the traffic volume forecasting process is separated from the main system and built on Microsoft Excel using Visual Basic Application (VBA) language for the purpose of increasing users' accessibility. All other analysis procedures are incorporated in TransCAD using its built-in macro language, GIS Developer's Kit (GISDK).

The framework of the modeling subsystem, showing inputs, process and outputs, is displayed in Figure 6.2.

A. Excel VBA interface

The Excel VBA interface calculates the factors required for traffic volume forecasting, including growth factors, directionality and peak period factors. One of the inputs is historical monthly ATR files, which include the road class of each ATR station and hourly volumes of both directions within the month. The other input, day type 
definition, is optional. If the day type definition is not specified, the default day type definition, weekdays and weekends, is used.

According to the methodology mentioned in Section 5.2, the ATR data are first screened for any apparent errors caused by malfunction of equipment. Then the data are grouped in three different ways for the purpose of calculating three different factors. The first grouping method is to group data by day types and road classes for calculating peak period factors. For each subset of the data, the peak periods are defined based on the maximum total volumes for three consecutive hours. The peak period factors are then calculated.

The second grouping method is to group data by day types, road classes and directions for calculating directionality. In accordance with the peak periods defined in the first grouping method, the directionality for the defined peak periods is calculated. After the peak period factors and directionality are obtained, the two values are combined as the adjusted peak period factors.

The third grouping method is to group data by day types and stations for calculating growth factors. First, the monthly average daily traffic (MADT) is calculated for each subset of data for each monthly ATR file. The process of MADT calculation is repeated for the remaining 11 months of the same year. Then, the annual average daily traffic (AADT) is calculated. The process of MADT and AADT calculations is then repeated for the adjacent year. Next, the growth factor for each subset is calculated. Finally, the growth factors are further grouped by road classes and calculate the average growth factors for each road class. If the calculation of growth factors is not performed, the 
latest available growth factors from 1998 to 1999 provided by the of Roadway Management Division of INDOT (Table 5.9) are used as the default values.

The flowchart of the Excel VBA interface is shown in Figure 6.3.

\section{B. TransCAD GISDK interface}

The TransCAD GISDK interface is used to verify the predicted delays caused by road construction and to generate a delay database for the use in travel information access subsystem. The required inputs of this subsystem include a network database, a peak factor file from the Excel VBA interface, a construction schedule database and an existing hourly volume file or a file name for storing hourly volumes. The network database should contain road classifications, ADTs and normal capacities. The peak factor file should contain peak period factors, directionality, growth factors and day type definitions. The construction schedule file should contain information on construction schedules and work zone capacities. An existing hourly volume file may contain the "no information" hourly volumes, or both the "no information" and the "complete information" hourly volumes. If the hourly volume file contains only the "no information" hourly volumes, the "complete information" hourly volumes will be generated when an initial O-D table is provided. The initial O-D table is an optional input that is used to perform lower bound delay analysis and delay refinements according to the age of the workzone. If an O-D table is not given, only the "no information" analysis may be performed and the upper bound of link delays will be displayed. If both the "no information" and the "complete information" hourly volumes exist in the hourly volume file, a complete delay analysis, including upper bound delays from the "no information" analysis, lower bound delays from the "complete information" analysis, and 
adjusted delays, can be performed, and a delay database can be generated. Users may also choose to overwrite the hourly volumes based on current inputs.

The TransCAD GISDK interface first takes the outputs of the Excel VBA interface and the network database to calculate the "no information" link volumes, the upper bound on hourly volumes. The values of the "no information" link volumes are to simulate the traffic conditions of the "no information" scenario when no highway users know that the workzone exists.

Next, the "no information" link volumes obtained from the above process are used with an initial hourly O-D table to estimate the O-D tables for different time periods. Each cell in the estimated O-D tables represents the demand for that O-D pair. Then the estimated O-D tables and road construction schedules obtained from the project scheduling subsystem are used to generate the "complete information" link volumes (the lower bound on hourly volumes) by performing traffic assignment. The "complete information" link volumes are to simulate the "complete information" scenario, in which all highway users know about the existence of the construction zones.

The "no information" and the "complete information" link volumes are used as the inputs for estimating delays under the "no information" and "complete information" scenarios, and for obtaining adjusted link volumes based on the age of the construction zone. The adjusted link volumes are used to obtain the estimated delays in related to the age of the construction zone.

The flowchart of the TransCAD GISDK interface is shown in Figure 6.4. The process before "Generate delay database" represents a complete cycle for a single day type and a single set of construction projects working at the same time. Therefore, both 
the "no information" and the "complete information" volume analyses need to be repeated for each day type, once a set of construction projects active at the same time has been identified.

The number of times the "no information" volume analysis needs to be repeated is the product of the number of day types and the number of peak periods. Because the "no information" volume analysis is to simulate the "no information" scenario, travelers make decisions without knowing that workzones exist. Therefore, the interactive effects of multiple projects working at the same time does not need to be considered. The day types include the two default day types (weekdays and weekends) and special days defined by modeling personnel. The peak periods include the three periods of AM-peak, PM-peak and off-peak.

The "complete information" volume analysis involves two processes: O-D estimation and traffic assignment, as shown in Figure 5.1. The number of times to repeat O-D estimation is the number of times the "no information" volume analysis is repeated for all day types subtracted by the number of times for weekends, because O-D estimation generates demands for different day types from the "no information" link volumes except weekends. The traffic patterns for weekends are estimated from the "no information" analysis.

The number of times traffic assignment needs to be repeated depends on the number of day types except weekends, the number of time periods in a day and the number of different sets of construction projects active at the same time. Because traffic assignment simulates drivers" choices under the "complete information" scenario, the existence of different sets of projects working simultaneously may have different effects if some of 
the projects are close to each other. Figure 6.5 shows how different time spans are defined by the different sets of construction projects that are active at any given time. Whenever a construction project starts or ends, the number of construction projects active simultaneously changes. The number of time spans in a specific month is the number of times different projects start or end within the month plus 1 . The first time span always starts at the first day of the month. For example, Figure 6.5 shows five time spans. The time span $T_{1}$ involves two projects, $X$ and $Z$. The time span $T_{3}$ involves four projects, $W$, $\mathrm{X}, \mathrm{Y}$ and $\mathrm{Z}$. The number of applications of traffic assignment in each time span is determined by the number of different day types within the time span. For example, the number of applications of traffic assignment in the time span $T_{1}$ is 1 (for weekdays). The number of applications of traffic assignment is to be repeated for time span $T_{3}$ is 2 - one for weekdays, and the other for the special day within the time span. The number of times traffic assignment is to be repeated is the sum of two products. The first product is from the number of special day types and the three periods (AM-peak, PM-peak and offpeak). The second is the product of the three periods (AM-peak, PM-peak and off-peak) for weekdays and the number of different dates whenever a project starts or ends plus 1 (the first day of the specific month).

After finishing the "no information" and the "complete information" volume analyses, a relationship between hourly volumes and the age of construction found in historical data is applied to obtain adjusted link volumes according to the age of construction. The delays caused by workzones during the transition time are estimated. These delays provide the project scheduling personnel an idea of the traffic conditions under the existing project strategy. As explained at the beginning of this chapter, there 
exists an interactive effect between the modeling subsystem and the project scheduling subsystem. The construction schedule can be adjusted based on the estimated delays, and the delays are re-estimated according to the modified construction schedule. The iterative process between the modeling subsystem and the project scheduling subsystem is repeated until both the project schedule and its causing delays are considered to be acceptable. The final estimated delays are stored in a database in dBaseIV format.

Before the dBaseIV delay database can be provided for public access, the database needs to be converted into a format that is accepted by the web server of the travel information access subsystem. Currently, the temporary web server of the access subsystem only supports the database in Microsoft Access format. The dBaseIV delay database needs to be converted into the format of Microsoft Access before being uploaded to the web server.

\subsection{Project Scheduling Subsystem}

The project scheduling subsystem is a graphic users' interface database management system implemented in the TransCAD GISDK interface. The function of this subsystem is to manage the database of construction schedules. The required inputs are a network file and an existing construction schedule file or a file name for storing construction schedules. A network file should include node coordinates. An existing construction schedule file should include information on workzone schedules and work zone capacities. The optional inputs are an hourly volume file, a peak factor file and an O-D matrix file. These files are for use of checking the effects of current construction scheduling strategy on the traffic conditions of the corresponding highway sections. 
The project scheduling subsystem provides some basic database manipulation functions such as "Add", "Delete" and "Save edited", and evaluation of the current scheduling strategy such as "Link delay" for checking single link delay and "Evaluate schedule" for checking system performance.

With the database manipulation functions, the project scheduling personnel may add, delete and edit records by clicking on a link on the map. Each record contains information on construction: project prefix, project number, beginning date, end date, number of lane closures and a brief description about the construction. When an existing construction schedule file is retrieved, both the records and the locations will be displayed in a table and on the map.

The functions for evaluating current scheduling strategy allow the project scheduling personnel to check its effects on traffic and to adjust schedules accordingly. When the function of "Link delay" is selected, this subsystem is linked back to the main menu of the modeling subsystem for volume and delay analyses according to the current scheduling strategy. When the function of "Evaluate schedule" is selected, the project scheduling personnel are asked to input the evaluation period before directed back to the modeling subsystem. These two functions are disabled if the inputs for the "no information" and/or the "complete information" volume analyses are not given.

When the objective is to evaluate current scheduling strategy, the system determines the total delay for the current scheduling strategy. If the traffic delay is unacceptable, users of project scheduling may go back to the main menu of the project scheduling subsystem to modify the construction schedule. This function of schedule evaluation is 
also disabled if the inputs for the "no information" and/or the "complete information" volume analyses are not given.

\subsection{Web Access Subsystem}

The function of the web access subsystem is to provide highway travelers a userfriendly and easily accessible way to retrieve travel information for trip planning. This subsystem is built on Hyper Text Markup Language (HTML), Javascript language, and Microsoft Active Server Pages (ASP) scripting using VB-script language. HTML is the standard language for web page editing. Javascript language is used to dynamically generate options of the departure date on the web page to avoid users' input errors and to dynamically show detailed information about a specific construction project upon users' requests. Currently, this subsystem is designed to provide travel information to drivers between the current date and the end of next month. If construction schedules can be set more than two months in advance, more months of travel information can be provided.

ASP is a server-side scripting, which re-generates documents each time they are accessed. With ASP, the server gets a chance to alter the file before sending it to the user. Each time a users submits a different departure time, the corresponding delay information from the database of estimated delays is translated into HTML pages and then sent back to the user. Therefore, this access subsystem does not require any platform-dependent software to operate on. Any users with access to the Internet and a browser can retrieve the travel information from this access subsystem.

The web access subsystem allows users to submit their time of departure input with a web browser to ASP, which retrieves the corresponding delay information from the 
database of estimated delay stored on the server and sends the information back to users on their browsers.

One important characteristic of the web access subsystem is its instant response to a user's query. The delay information is generated in the modeling subsystem and stored in a database. The process time for the web access subsystem is the time of data transmission on the Internet. The response time to a user's query with a $56.6 \mathrm{Kbps}$ modem is within seconds. Users may perform several queries within to consider alternate trip plans.

\section{$\underline{6.4}$ System Maintenance}

The purpose of system maintenance for the HTIS is to keep the travel information updated through routine operation of the system. As explained at the beginning of this chapter, the HTIS is divided into three subsystems: modeling, project scheduling, and web access. These three subsystems operate separately, yet are bonded with one another. Some of the outputs for one subsystem are the inputs for another. Therefore, the changes in one subsystem may result in the changes to others. The interactions among the three subsystems should be considered in the system maintenance program.

The following paragraphs explain the tasks involved in the maintenance for each subsystem, the personnel who take charge of those tasks, the update frequency and the operation time for each update process. 
A. Maintenance of the modeling subsystem

Two maintenance tasks are involved in the modeling subsystem - maintenance of the Excel VBA interface and maintenance of the modeling task in the TransCAD GISDK interface.

(1) Maintenance of the Excel VBA interface

The task of maintaining the Excel VBA interface is to generate the files of peak period factors. Each file of peak period factors contains the definitions of day types for a specific month, the definitions of peak periods and the values of peak period factors for different day types and road classes for the month, and the values of annual growth factors for different road classes for the month. All of the items, except the annual growth factors, should be generated through the Excel VBA interface. The annual growth factors can be obtained from the Roadway Management Division of INDOT. The Excel VBA interface is part of the modeling process. The personnel familiar with the use of the statewide model should be expected routinely operate the Excel VBA interface.

The frequency of maintaining the Excel VBA interface depends on the characteristics of the inputs for the interface. The required inputs for the Excel VBA interface are the files of ATR data, which can be obtained from the Roadway Management Division of INDOT. Each ATR data file stores hourly volumes of each ATR station for a specific month in a specific year. Based on the assumption that the traffic patterns of a specific month in different years are similar, a historical ATR data file of a specific month can be used to generate the peak period factors for the same month, which can be used to represent the traffic patterns of the same month in any future year. Although the peak period factors of a specific month based on an old ATR data file can serve the same 
purpose of reflecting the traffic patterns of the same month, the ones based on a more recent ATR data file can depict more up-to-date traffic patterns. Therefore, the peak period factors for a specific month are suggested to be updated based on the latest available ATR data file of the same month.

The update frequency for the Excel VBA interface is determined by the time of obtaining the latest available ATR data files, the needs of updated peak period factors and the preferences of the personnel who are in charge of the updates. For example, the latest available ATR data file for December 1999 is obtained in July 2000. According to the current settings of the HTIS, the system should provide travel information for the remainder of current month and the entire next month. The peak period factors should be generated at least two months in advance, which in the above example is by October 2000. Assume the first preference of the personnel is to update the peak period factors for a specific month whenever the new latest ATR data file of the same month is available, and the second preference of the personnel is to update peak period factors of multiple months at the time when the peak period factors of the first month among the multiple months are needed. In the above example, the time of updating the peak period factors of December is in July 2000, based on the first preference and in October 2000, based on the second preference, because the peak period factors for December 2000 are needed by October 2000. Assume three more ATR data files - the files of January, February and March 2000 - will be available by October 2000. The peak period factors for the four months will be updated in October 2000. The average update frequencies, according to the two preferences of the personnel are once a month and three times a year (12 months / 4 months of data per time). The maintenance personnel may choose any 
frequency between once a month for updating peak period factors of a month and three times a year for updating peak period factors of four months in the above example.

The run time for each ATR file on a PC with a Pentium III - 266 CPU is less than 2 minutes.

(2) Maintenance of the modeling task in TransCAD GISDK interface

The update frequency for the modeling task in the TransCAD GISDK interface depends on the number of updates required for its inputs and the preference of the personnel who are in charge of maintaining the modeling task of this interface. There are four required inputs and one optional input for this task. The required inputs include peak period factors, a network file, project schedules and a file name of hourly volumes. The optional input is an initial O-D table, which is for the analysis of the "complete information" scenario. Among these inputs, the file name of the link hourly volumes is for the storage of the intermediate results, therefore, it does not affect the update frequency of this task. The network file and the initial O-D table, which are available from the INDOT, change only at a major update for the entire system. The two inputs changing frequently are peak period factors and project schedules, both of which are the required inputs for this task. The peak period factors differ for each month under the assumption that the traffic patterns of each month can be represented by a unique set of peak period factors that are stored in a separate file. Each file of peak period factors contains several subsets, each of which represents the traffic patterns for a specific day type in a specific month. The number of updates required for each set of peak period factors equals the number of day types in the month. The project schedules, on the other hand, do not change on a regular basis. Each project schedule is considered individually 
and the start date adjusted to reduce the impacts the project on the traffic, if other factors permit. Whenever a project schedule is changed, the modeling task needs to be performed to update the traffic conditions based on the new schedule. The number of updates required for the modeling task is the product of the numbers of updates for the peak period factors and the project schedules. For example, the number of updates required for the peak period factors in a specific month is two, the number of day types in the month is two and there is only one project involved. The schedule of the project is adjusted seven times including the first time it is added into the schedule database. Three of the seven adjustments are to reschedule to the specific month. The number of updates required for the project schedule is three. Therefore, the number of updates required for the modeling task in the specific month is six $(=2 \times 3)$. The highest update frequency for this task is six times per month, which means one update per each time. Whenever the final update for the month is finished, the delay database for the month can be generated. The personnel who are in charge of this task may choose a lower update frequency as long as the delay database for the month can be generated in time for public access. The time needed to generate delay databases is mentioned in "Maintenance of the web access subsystem" later in this section.

The run time for the modeling task depends on the number of updates required for the task, the provision of the optional input and the choice of generating a delay database. The number of updates required for the task with respect to the modeling task has been explained in the previous paragraph. The input of an initial O-D table is for the analysis for the "complete information" scenario. If an initial O-D table is not provided, only the "no information" analysis is performed. The run time for the "no information" analysis 
per each update on a PC with a Pentium III - 266 CPU is about three minutes. If an initial O-D table is provided, the "no information" and the "complete information" scenarios are both analyzed. The run time for the "complete information" analysis per each update on the same PC is about fifty minutes. The run time for generating a delay database on the same $\mathrm{PC}$ is about thirty minutes. The total run time for all updates is the run time per each update multiplied by the number of updates required plus the run time for generating a delay database.

B. Maintenance of the project scheduling subsystem

The task of project scheduling is implemented in the TransCAD GISDK interface. The update frequency for the project scheduling task depends on the number of updates required for its inputs and the preference of the personnel who are in charge of this task. There are two required inputs and three optional inputs for this task. The required inputs include a network file and project schedules. The optional inputs include peak period factors, a file name of hourly volumes and an initial O-D table, which are for delay analyses based on the current scheduling strategy. As explained in "Maintenance of the modeling task in TransCAD GISDK interface" previously in this section, the network file and the initial O-D table change only at a major update for the entire system. The file name of the link hourly volumes does not affect the update frequency for this task because it is for the storage of the intermediate results. The only inputs affecting the update frequency for this task are peak period factors and project schedules. Both the numbers of updates for these two inputs have been explained in "Maintenance of the modeling task in TransCAD GISDK interface" previously in this section. The update frequency for the project scheduling task is similar to that for the modeling task. The 
difference between these two tasks is that the peak period factors are an optional input for the project scheduling task. If the peak period factors are not provided, the project scheduling task is not linked to the modeling task and the only thing involved in the project scheduling task is the database manipulation for the schedule database. The update frequency for the project scheduling task in this case depends only on the number of updates required for the project schedules. For the example in "Maintenance of the modeling task in TransCAD GISDK interface" previously in this section, the number of updates for the project scheduling task is three. The highest update frequency for the project scheduling task is three times per month, with one update each time. If the peak period factors are provided, the modeling and project scheduling tasks are linked together and the update frequency for the project scheduling task can be determined in the same way as for the modeling task in TransCAD GISDK interface, the higher frequency between the changing frequencies for the peak period factors and the project schedules.

The run time for the project scheduling task also depends on the number of updates required for this task and the provision of the optional inputs. The optional inputs, peak period factors, a filename for storing hourly volumes and an initial O-D table, are for delay analyses based on the current project scheduling strategy. If no optional inputs are provided, only the database manipulation for the schedule database is performed. The run time for each database manipulation is within one second. If some or all of the optional inputs are provided, the project scheduling task is linked to the modeling task and both the database manipulation and delay analyses for evaluating the current scheduling strategy are performed. The run time for the delay analyses is the same as that for the modeling task. When all three optional inputs are provided, the run time for 
the delay analyses is the same as the sum of the run times for the "no information" and "complete information" analyses. When the peak period factors and the file name for storing hourly volumes are provided, but the initial O-D table is not provided, the run time for the delay analyses is the same as that for the "no information" analysis.

C. Maintenance of the web access subsystem

The task for maintaining the web access subsystem is to keep travel information updated by uploading the update delay database on the Internet. The personnel in charge of web publishing should routinely update the delay database.

The update frequency for the web access subsystem is determined by the frequency of generating the updated delay database and the preference of the personnel who are in charge of the maintenance of this subsystem. The delay database is one of the outputs from the TransCAD GISDK interface. To provide continuous access to the public, the delay database is divided into multiple files. Each file contains the delay information for a specific month. In this way, the delay database for a specific month can still be accessed while the file of the delay database for the next month is being uploaded. As explained in the update frequency for the modeling task in TransCAD GISDK interface, the delay database for a specific month can be generated when the final update in the month for the modeling task is finished. The personnel in charge of the maintenance of the web access subsystem may upload the updated delay database any time after it is generated. However, in order to provide sufficient travel information in advance, the upload process for the delay database of a specific month should be no later than two months before the specific month, if the adjustment of the project schedules for the month is finished at least two months in advance. Travel information with a greater 
"horizon" can be provided if the project schedules for a specific month can be determined more than two months before the specific month.

The run time for uploading a delay database depends on the transmission speed used to access the Internet. The time for uploading a delay database on a $56.6 \mathrm{~K}$ modem is about ten minutes. 


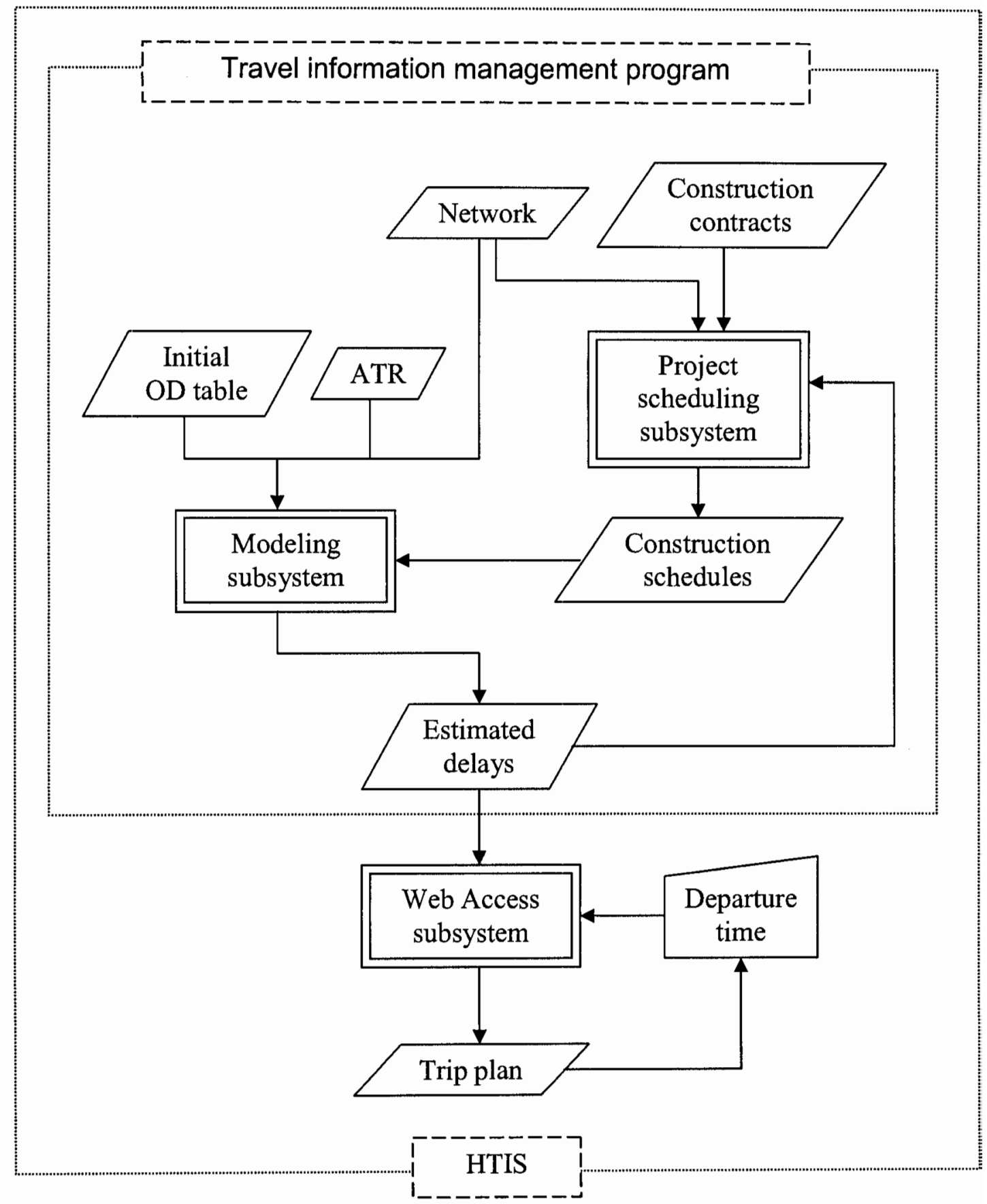

Figure 6.1 Relationships among three subsystems of HTIS 


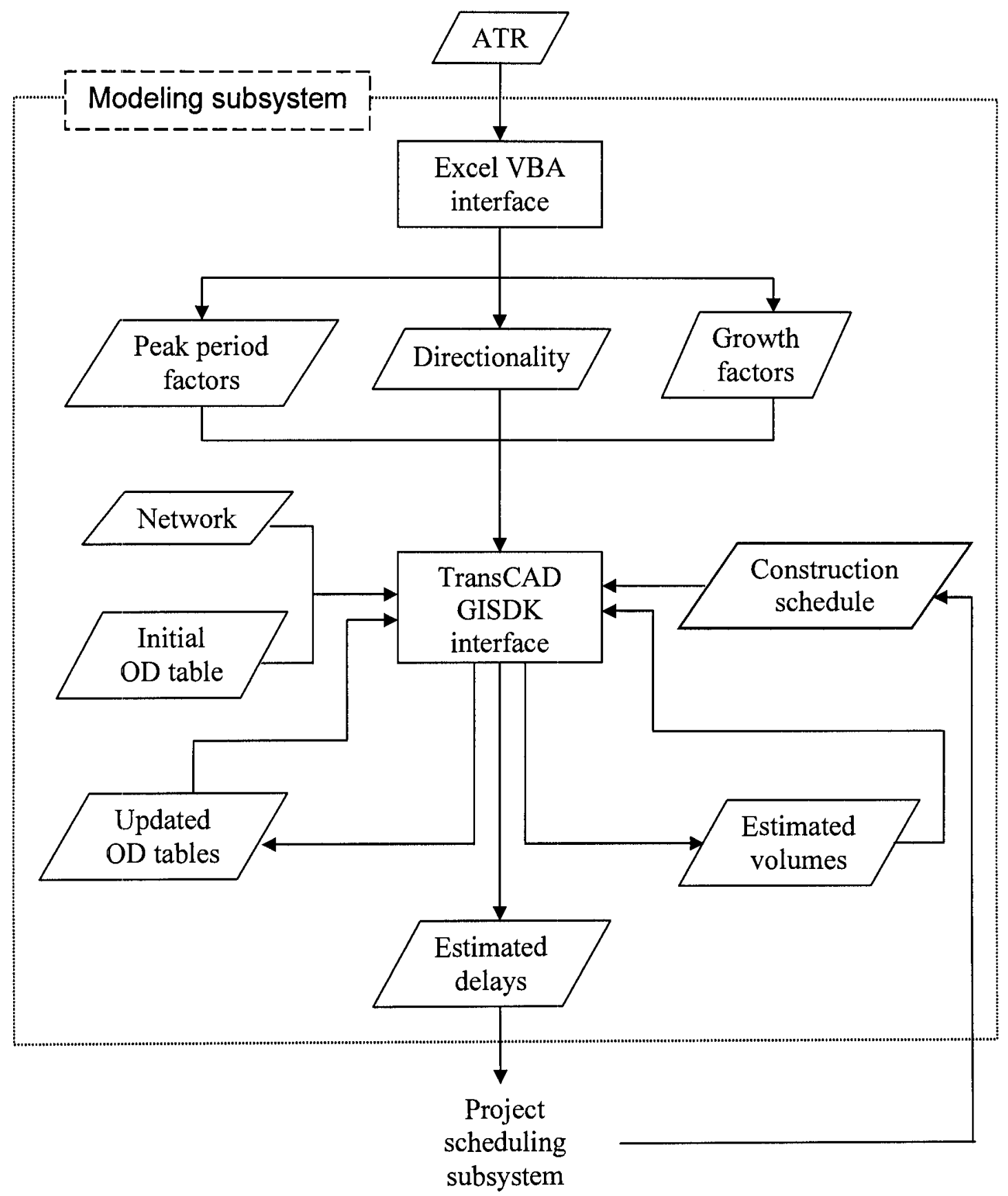

Figure 6.2 Framework of the modeling subsystem 


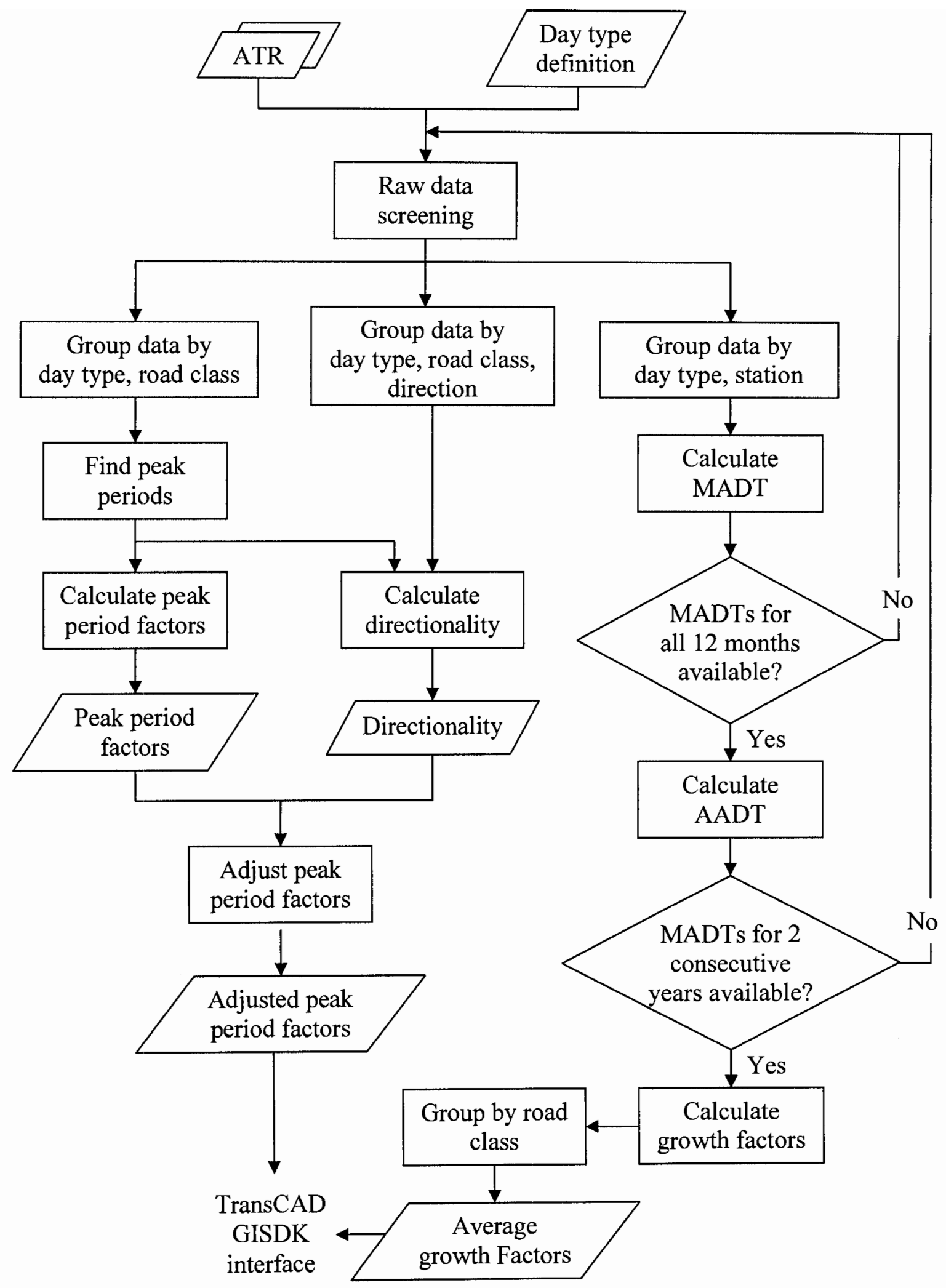

Figure 6.3 Flowchart of the Excel VBA interface 


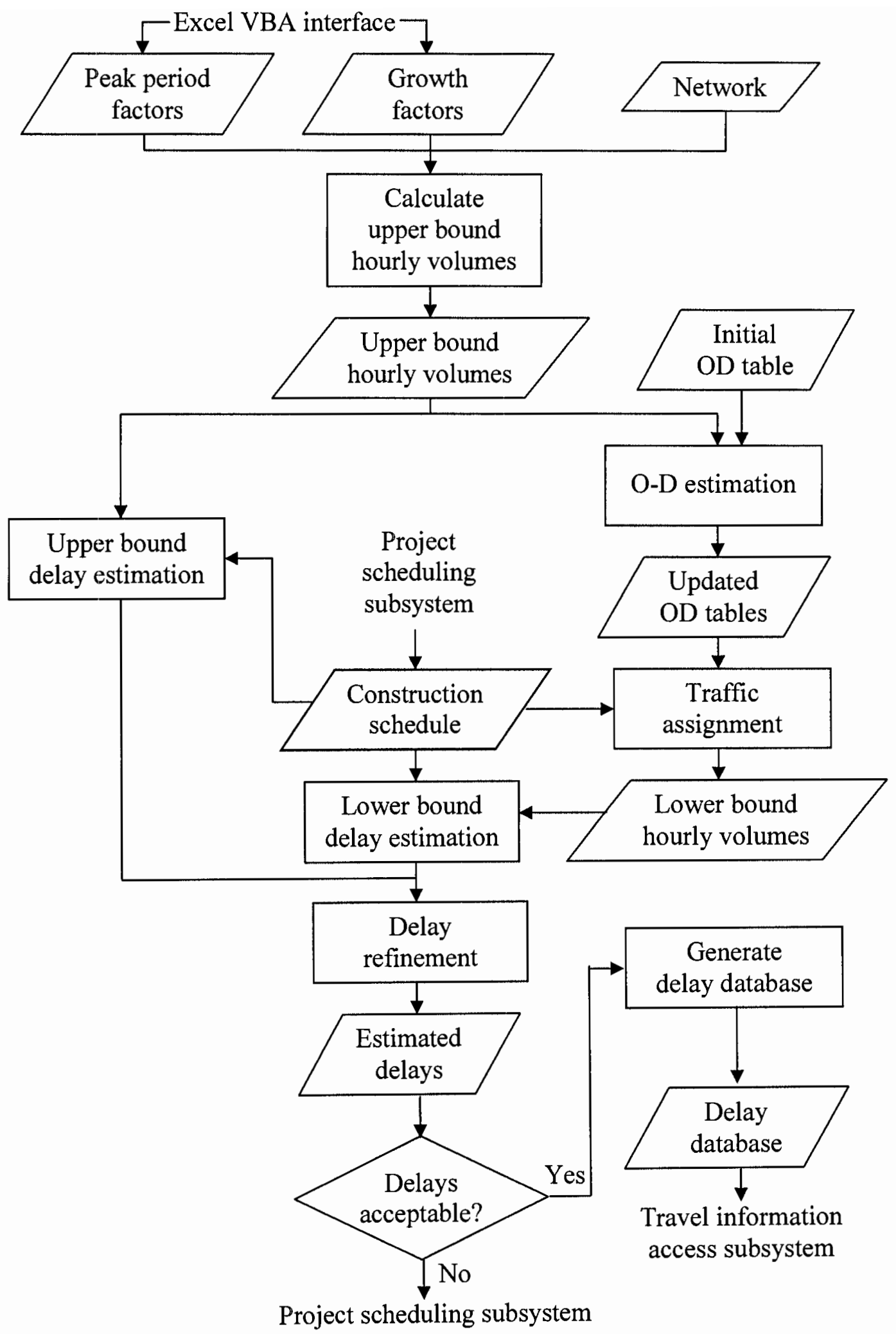

Figure 6.4 Flowchart of the modeling subsystem's TransCAD GISDK interface 


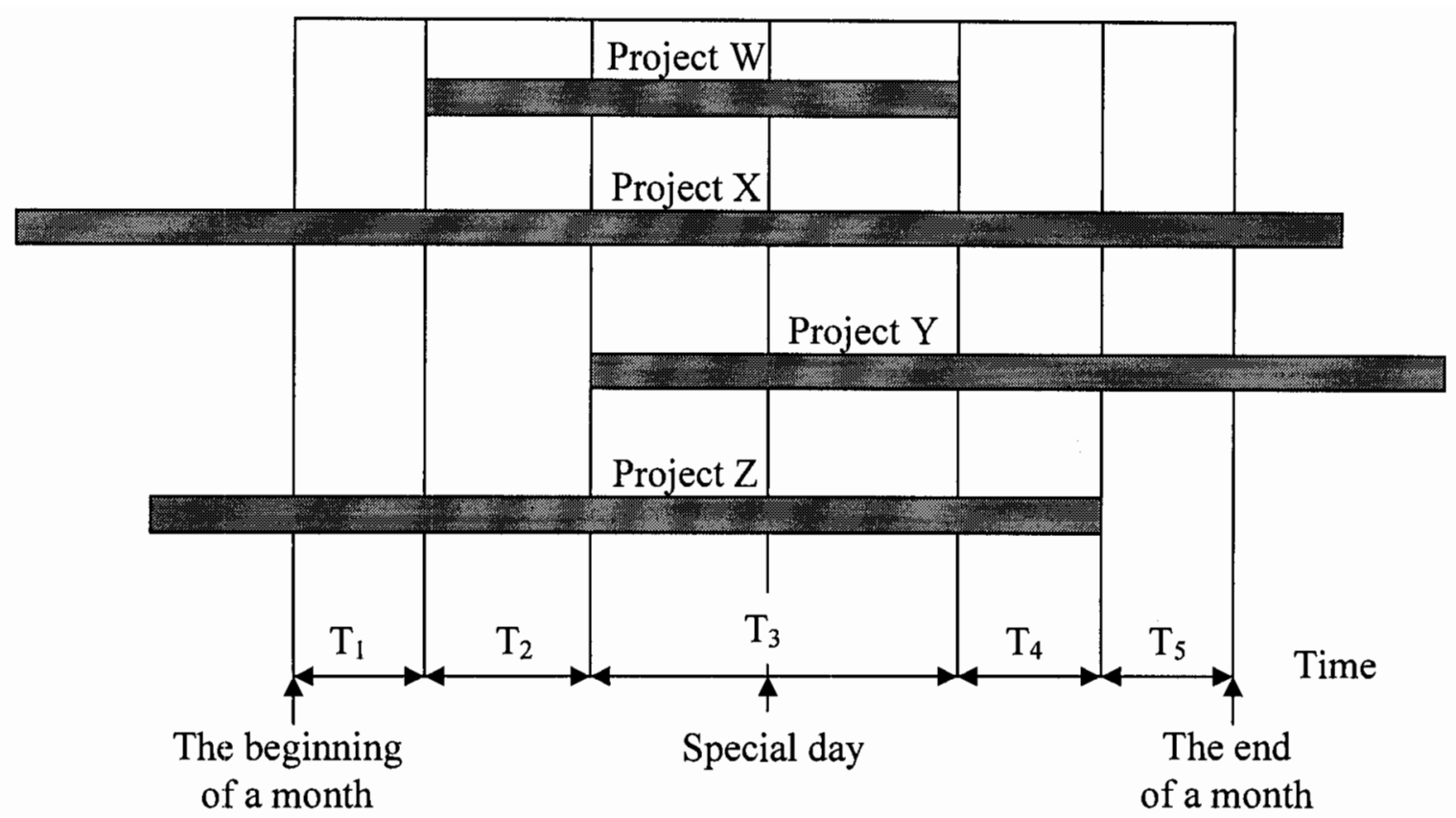

(Note) Project duration

$\mathrm{T}_{1} \sim \mathrm{T}_{5}$ : Time spans involving different sets of projects

Figure 6.5 Time span definitions for traffic assignment 


\section{SUMMARY AND FINAL COMMENTS}

\subsection{Summary}

Travel information systems can be divided into three groups - real-time, short-term pre-trip and long-term pre-trip. The real-time information systems provide drivers information during their trips, short-term pre-trip information systems shortly before departure (normally within one day), and long-term pre-trip information systems one day or more before departure. The real-time and short-term pre-trip information systems are suitable for providing information about unexpected or nonrecurring events such as incidents, while the long-term pre-trip information systems are designed to provide information about expected events such as road construction. Previous research about travel information systems has focused on analyzing and publicizing the effects of unexpected events on traffic. Little attention has been given to long-term pre-trip information systems because the real-time and short-term pre-trip information systems can model both unexpected and expected events. However, because of the complicated attributes of unexpected events, real-time and short-term pre-trip information systems need further development to be effective in serving motorists. Long-term pre-trip information systems can coexist with real-time and short-term pre-trip information systems by meeting travelers' needs at different times. Travelers with flexible schedules may check with the long-term pre-trip information systems may schedule their trips that 
require be set several days in advance and adjust trip plans at the time departure and during driving according to the real-time and short-term-re-trip information systems for the purpose of minimizing possible delays.

This study developed a long-term pre-trip information system called the Highway Travel Information System (HTIS) by using an approach called Workzone Delay Equilibrium Estimation (WDEE). WDEE attempts to find a new "steady state" for delay based on the level of traffic that will use the workzone link after its existence has become widely known. WDEE involves three steps - analysis for the "no information" scenario, analysis for the "complete information" scenario and estimation of the delays during the construction period. The WDEE process also allows the HTIS to estimate workzone traffic flows (and therefore delays) for any time during the transition to the new steady state.

\section{A. Analysis for the "no information" scenario}

The "no information" scenario simulates the condition at the beginning of a road project when no drivers know that the new construction zone exists. Drivers will follow the same routes they normally use during the same time frame, unaware of any newly constrained capacities on some links on their routes. In the "no information" scenario, the traffic flows on each link for a specific time frame at the beginning of road construction are treated the same as when there was no construction. By applying proper traffic volume forecasting techniques, the volumes on over 16,000 links in the Indiana highway network can be estimated. Traditional traffic volume forecasting techniques are not suitable for use in the HTIS because they require detailed data and long execution time. A simplified traffic volume forecasting technique was developed for the purpose of 
estimating flows on a large number of links. The main advantages of the simplified traffic volume forecasting technique are that it can be applied to links without detailed data and it can be applied to large network such as the Indiana statewide network. B. Analysis for the "complete information” scenario

The "complete information" scenario simulates the case in which road construction has been proceeding long enough that all drivers using that road know about it. Drivers make informed decisions about whether to stay on the original route or divert to alternate routes, based on their knowledge of the workzone and the surrounding network. Under the assumption that drivers will only divert to alternate routes (rather than change their departure time) to minimize delays, the travel demand between each O-D pair for a specific time period will be the same for both the "complete information" and "no information" scenarios. The demand for trips between each O-D pair for a specific time frame can be estimated through the O-D estimation process using the "no information" link volumes and the a priori matrix as inputs. This demand for trips between each O-D pair is then assigned to a network having work zone capacity constraints to obtain the link flows for the "complete information” scenario.

C. Estimation of the delays during the construction period

The actual traffic conditions during construction periods are in between the "no information" and the "complete information" scenarios. The volumes at a workzone are expected to decrease as construction proceeds and more drivers become aware of the construction. The volumes stop decreasing as the system reaches an equilibrium state. Because there are always a certain percentage of infrequent travelers that are not aware of the construction, the volumes at the equilibrium state are higher than the "complete 
information" link volumes. The relationship between the historical data and the age of construction is hypothesized using a logistic function. This hypothetical relationship is applied to the "no information" volumes and the volumes at the equilibrium state. The link volumes at any time during the construction period can be, therefore, estimated.

The delays during the construction period are estimated using queueing analysis. The service rate is the hourly workzone capacity based on the study of Dudek and Richards (1981) and the arrival rate is the estimated link volumes obtained by applying the hypothetical relationship to the two extreme link volumes (the "no information" and “complete information” volumes). Knowing how the arrival rate is likely to change the early stages of construction, the HTIS can estimate average delay per vehicle as more drivers become aware of it.

The HTIS is divided into three subsystems - modeling, project scheduling, and web access. The modeling and project scheduling subsystems are for management purposes and the web access subsystem is for use by the traveling public. The modeling subsystem is built with the Excel VBA and the TransCAD GISDK interfaces. The Excel VBA interface implements the simplified traffic volume forecasting technique developed for the HTIS. The TransCAD GISDK interface performs WDEE, which includes the analysis of the "no information" scenario, the "complete information" scenario, the estimation of link volumes during the transition construction period, delay estimation and delay database generation. The project scheduling subsystem is also built on the TransCAD GISDK interface. There are two major tasks for the project scheduling subsystem. The first one is to manage project schedule databases. The second task is to assist project scheduling personnel in determining proper scheduling strategies to avoid 
excessive traffic delays by providing functions to adjust project starting date, end date, and lane closure strategies in terms of workzone capacities. The web access subsystem is built on several scripting languages, including Hyper Text Markup Language (HTML), JavaScript, VB-script, and Active Server Pagers (ASP). The JavaScript and VB-script provide an interactive environment. The ASP is a server-side-include scripting language, which can generate personalized travel information upon receiving users' requests. Travelers may input the proposed departure time to retrieve information about expected delays at workzones that are active at the departure time and use the information to plan their trips up to two months in advance.

The HTIS requires routine maintenance in order to provide updated travel information. Most of the maintenance tasks are accomplished during routine use of the subsystems.

\section{$\underline{7.2 \text { Final Comments }}$}

The HTIS can be further refined to improve the accuracy of workzone delay estimates and to enhance the function of assisting project scheduling in the following five ways.

(1) New techniques in traffic volume forecasting, traffic assignment, O-D estimation, and workzone delay estimation may be applied in the HTIS. Because the architecture of the HTIS is highly modularized, each part of the analysis in the modeling subsystem of the HTIS is independent from others. New techniques for any of the parts can be plugged into the HTIS easily without changing an other part of the HTIS. However, the application of new analysis techniques needs to consider the availability of required data and the feasibility of applying it on a large network. 
(2) The accuracy of workzone delay estimates can be further verified. Only two surveys were conducted to validate workzone delay estimates (both on I65 between SR43 and SR18). The results were satisfactory, but the accuracy of the delay information generated by the HTIS would benefit from further verification.

(3) The effects of information available via the World Wide Web (WWW) on travelers' behavior require further study. Currently the WWW has not been used to disseminate information about workzone delays. Travelers make decisions based on the information provided via traditional media such as TV, radio and variable message signs (VMS). The information provided by the HTIS via the WWW may affect travelers' behavior to different degrees. By understanding the aggregate effects of the WWW on the traffic, the HTIS may provide more accurate delay estimates.

(4) The function of assisting project scheduling can be further extended. The information provided in the HTIS for assisting project scheduling is the total delay based on current scheduling strategy. A sensitivity analysis of project starting dates, end dates and lane closure strategies on system performance may be added.

(5) Observed data about hourly volumes during the construction period can be used to analyze the relationship between hourly workzone approach volumes and the age of construction. The plan was to take advantage of the continuous data provided by the Automated Traffic Recorder (ATR) stations by finding construction sites located downstream of the ATR sites. However, no suitable data for such cases have been obtained so far. In the rare cases in which an ATR site was immediately upstream from a workzone, equipment malfunctions prevented a detailed analysis of traffic volume trends. An approximate relationship between hourly volumes and the age of 
construction has been hypothesized. The relationship between hourly volumes and the age of construction based on observed data may depict the aggregate effects of travelers' behavior during the construction period more accurately. 


\section{LIST OF REFERENCES}

Abraham B. and Ledolter J. (1983) Statistical methods for forecasting. John Wiley \& Son Ltd., New York.

Adler J. and Blue V. (1998) Toward the design of intelligent traveler information systems. Transportation Research Part C 6, pp.157-172.

Allen, Ziedman, Rosenthal, Stein, Torres and Halati (1991) Laboratory assessment of driver route diversion in response to in-vehicle navigation and motorist information systems. Transportation Research Record 1306, pp.82-91.

Arnott R., de Palma A. Lindsey R. (1991) Does providing information to drivers reduce traffic congestion? Transportation Research Part A, Vol. 25A, No. 5, pp.309-318.

Barfield W., Haselkorn M., Spyridakis J. and Conquest L. (1991) Integrating commuter information needs in the design of a motorist information system. Transportation Research Part A, Vol. 25A, Nos. 2/3, pp.71-78.

BGC (1993) FMC documentation, v. 2.10, Bureau Goudappel Coffeng bv, Deventer, The Netherlands.

Boruff D. (1994) An investigation into the effects of highway construction on driver behavior. Indiana Department of Transportation. Indianapolis, IN.

Caliper Inc. (1999) Travel Demand Modeling with TransCAD 3.5. Newton, MA.

Cambridge Systematics, Inc. (1998) Major Corridor Investment-Benefit Analysis System: Model Documentation Part I: Indiana Statewide Travel Model.

Cassidy M. and Han L. (1993) Proposed model for predicting motorist delays at two-lane highway work zones. Journal of Transportation Engineering, Vol. 119, No. 1, pp27-42.

Dixon K., Hummer J. and Rouphail N. (1998) Comparison of rural freeway work zone queue length estimation techniques: a case study. Transportation Research Board, $77^{\text {th }}$ Annual Meeting. Washington, D.C. 
Dougherty M. and Cobbett M. (1997) Short-term inter-urban traffic forecasts using neural networks. International Journal of Forecasting, Vol. 13, No. 1, pp.21-31.

Dudek C. and Richards S. (1981) Traffic capacity through work zones on urban freeways. Texas Transportation Institute. Research Report 228-6. College Station, Texas.

Dudek C., Richards S. and Buffington J. (1986) Transportation Research Record 1086, pp.20-30.

FHWA (1995) Traffic Monitoring Guide. FHWA-PL-95-031. Federal Highway Administration.

Hamerslag R. and Immers B. (1988) Estimation of trip matrices: shortcomings and possibilities for improvement. Transportation Research Record 1203, pp.27-39.

HCM (1997) Highway Capacity Manual, Special Report 209, $3^{\text {rd }}$ edition. Transportation Research Board. Chapters 1 and 3.

Jha M. and Sinha K. (1996) Effective scheduling of road and bridge closures: phase II. Joint Highway Research Project. FHWA/IN/JHRP-96/7. Purdue University.

Jiang Y. (1999) Traffic characteristics and estimation of traffic delays and user costs at Indiana freeway work zones. Indiana Department of Transportation, Division of Research. FHWA/INDOT/SPR-2121. West Lafayette, IN.

Khattak A., Schofer J. and Koppelman F. (1995) Effect of traffic information on commuters' propensity to change route and departure time. Journal of Advanced Transportation, Vol. 29, No. 2, pp.193-212.

Khattak A., Yim Y. and Stalker L. (1999) Does travel information influence commuter and noncommuter behavior? Transportation Research Record 1694, pp.48-58.

Kirby H., Watson S. and Dougherty M. (1997) Should we use neural networks or statistical models for short-term motorway traffic forecasting? International Journal of Forecasting, Vol. 13, No. 1, pp.43-50.

Lam W. and Lo H. (1990) Accuracy of O-D estimation from traffic counts, Traffic Engineering Control, Vol. 30, No. 6, pp.358-367.

Memmott J. and Dudek C. (1982) A model to calculate the road costs at work zones. Texas Transportation Institute. Research Report 292-1. College Station, Texas.

Ortuzar J. and Willumsen L. (1996) Modelling Transport, 2nd Edition. John Wiley \& Son Ltd., New York. Section 10.2 10.5, 10.7 and 12.4. 
Pavlis Y. and Papageorgiou M. (1999) Simple decentralized feedback strategies for route guidance in traffic networks. Transportation Science, Vol. 30, No. 3, pp.264-278.

Polydoropoulou A., Ben-Akiva M. and Kaysi I. (1994) Influence of traffic information on driver' route choice behavior. Transportation Research Record 1453, pp.56-65.

Nemeth Z. and Rouphail N. (1982) Lane closures at freeway work zones: simulations study. Transportation Research Record 869, pp.19-25.

Nielsen O. (1993) A new method for estimating trip matrices from traffic counts. Paper 1993-3, Institute of Roads, Traffic and Town Planning, the Technical University of Denmark.

Robertson D., Palumbo N. and Rice R. (1995) Impact of expressway reconstruction on adjacent traffic operations. ITE Journal, Vol. 65, No. 8, pp.43-53.

Scheaffer R. and McClave J. (1990) Probability and statistics for engineers, 3rd Edition. PWS-KENT Publishing Company. pp.585-592, 668.

Sefton D. (2000) Daily web surfing now the norm. Retrieved August 24, 2000 from the World Wide Web: http://www.usatoday.com/life/cyber/tech/cth591.htm

Smith B. and Demetsky M (1997) Traffic flow forecasting: comparison of modeling approaches. Journal of Transportation Engineering, Vol. 123, No. 4, pp.261-266.

Soares R. and Najafi F. (1998) User costs at the work zone. Transportation Research Board, $77^{\text {th }}$ Annual Meeting. Washington, D.C.

Thatcher A, Kannisto V, and Vaupel J (1998) The Force of Mortality at Ages 80 to 120. Odense University Press, Denmark. Chapter 2.

The CQ Researcher (1999) Congestion Is Increasing. The amount of severe congestion more than double from 1982 to 1996. The CQ Researcher, Vol. 9 No. 32, pp.735-744.

Yun S., Namkoong S., Rho J., Shin S. and Choi J. (1998) A performance evaluation of neural network models in traffic volume forecasting. Mathematical and Computer Modelling, Vol. 27, No. 9-11, pp.293-310.

Voort M., Dougherty M. and Watson S. (1996) Combining Kohonen maps with ARIMA time series models to forecast traffic flow. Transportation Research Part C, Vol. 4, No. 5, pp.307-318.

Wang K. and Elliott R. (1995) Design considerations for distributed, multimedia-based highway information system. Transportation Research Record 1505, pp.95-102. 
Whitford R. and Opsuth J. (1996) Congestion estimates for Indiana state highway system. Joint Highway Research Project. FHWA/IN/JHRP-96/8. Purdue University.

Yang J. and Fricker J. (1996) Estimating statewide trip tables from vehicle classification counts. Joint Highway Research Project. FHWA/IN/JHRP-95/15. Purdue University.

Zhang J., Leiman L and May A. (1989) Evaluation of operational effects of freeway reconstruction activities. Transportation Research Record 1232, pp.27-39. 


\section{APPENDIX \\ HIGHWAY TRAVEL INFORMATION SYSTEM USER'S GUIDE}

The application of the Highway Travel Information System (HTIS) is explained in three parts: the Excel VBA interface, the TransCAD GISDK interface, and the web access interface. The Excel VBA interface is for use by the modeling personnel. The TransCAD GISDK interface is for use by both the modeling and project scheduling personnel, and the web access interface is for use of public users (travelers). The relationship of these three users' interfaces is shown in Figure A.1. Through the Excel VBA interface, the peak period factors are generated and passed to the TransCAD GISDK interface. The delay database is then generated by the TransCAD GISDK interface and passed to the web access interface. Finally, public users may access the delay database through the web access interface.

Refer to Section 6.1 for development considerations of the Excel VBA interface and the modeling task in the TransCAD GISDK interface, to Section 6.2 for development considerations of the project scheduling task in the TransCAD GISDK interface, and to Section 6.3 for development considerations of web access interface. 


\section{A.1 Excel VBA Interface}

The Excel VBA interface has for two purposes: (1) To generate peak period factors, which will be stored in a dBase IV file. (2) To calculate monthly average daily traffic (MADT) for each ATR station. The framework of the Excel VBA interface is shown in Figure A.2.

Step 1: Enter the Excel VBA interface Enter the Excel VBA interface by double clicking on the icon "atrmacro.xls.” A message warning that the file contains macros will appear. Enable the macros, so that this program works properly. After this program has been entered, the introduction menu that briefly explains the functions of this program will appear on the screen (Figure A.3). By pressing the "Start" button, users can see the main menu for this program (Figure A.4).

Step 2: Inputs for the Excel VBA interface

The “Option” in the main menu allows the user to specify which function to execute: "generate peak period factors” or “calculate MADT.” The "generate peak period factors” function calculates peak period factors based on a file of Automated Traffic Recorder (ATR) historical data. The “calculate MADT” function obtains the monthly average daily traffic (MADT) for each ATR station. The inputs for these two functions are listed as follows:

(1) Inputs for the "generate peak period factors" function

- An ATR file, whose file name will be shown as the "Current ATR file";

- Additional inputs for generating peak period factors. (See Figure A.4.)

(2) Input for the "calculate MADT function” 
- A set of ATR files for up to 12 files: The file name of the ATR file that is being processed will be shown as the "Current ATR file."

Each input is explained below.

(1) Current ATR file:

The "Current ATR file” shows the ATR file that is being processed. By clicking the “Open ATR file(s)”, a dialog box will appear (Figure A.5). This program can handle two file types: the raw data in federal format (*.fed) and in Excel format (*.xls). The ATR files in federal format are obtained from the INDOT’s Roadway Management Division. These files contain a lot of rows of continuous text, whose entries are distinguished by column numbers (Figure A.6). Each row of data represents hourly volumes in a specific direction for a specific ATR station during a specific hour on a specific day.

If the ATR files in federal format are chosen, this program will transfer the files into the Excel format and save using the same name. For example, if the file "maytr98.fed" is chosen, it is transferred and saved as "maytr98.xls."

Users may select one ATR file if the option "Generate peak period factors" is chosen. If the option "Calculate MADT” is chosen, users may select up to 12 ATR files to process by pressing the "Control" key on the keyboard and selecting multiple files from the list of file names. When multiple files are chosen to be processed, the year and month of the ATR data in the file that is currently being processed are shown in the two textboxes next to the "Open ATR file(s)" button (Figure A.4). 
(2) Additional inputs for generating peak period factors:

This group of inputs is only required if the option is to generate peak period factors. If the option "Calculate MADT" is selected, this group of inputs is disabled. The inputs in this group include "Year of Departure", "Base Year of ADT", "Month of Departure”, “Enter Special Days” and “Annual Growth Factors.” They are discussed below.

- Year of Departure

The "Year of Departure" is for the calculation of the total growth factors, which are used to update the ADTs in the network database to the year of a trip's departure. An example input is like "2000.”

- Base Year of ADT

The "Base Year of ADT" is also for the calculation of the total growth factors of ADTs. Currently the ADTs in the network database are for the year 1995, the default value of the "Base Year of ADT." If the ADTs in the network database are updated, the default value of the "Base Year of ADT" should be updated accordingly.

- Month of Departure

The "Month of Departure" can not be changed by the user. The "Month of Departure" is automatically set to be the same as the month of the ATR data, based on the assumption that each month has its unique traffic patterns. For example, the ATR file for May 1998 can only represent the traffic patterns for May. 
- Enter Special Days

The “Enter Special Days” button allows the user to define day types in addition to the two default day types, weekdays and weekends. The special days are referred to as holidays and days with special events whose traffic conditions are different from normal weekdays and weekends. For example, special days can be Thanksgiving and days affected by the Indianapolis 500 car race. When clicking the “Enter Special Days” button, the special days input menu will appear (Figure A.7). Users can choose to specify special days by date or by day of week. Two examples of specifying special days are shown in Figure A.7. The first special day is specified by date, May 13. The second special day is specified by day of week, the third Sunday in May. This program allows entry of up to 10 different special days.

- Annual Growth Factors

The “Annual Growth Factors” input is required to calculate the total growth factor between the year of departure and the base year of ADT. The default values are the annual growth factors between 1998 and 1999 that are the latest available from the Roadway Management Division of INDOT (Table 5.9) as this project is completed. Users may edit these values if desired.

Step 3: Execute the Excel VBA interface

By clicking the "Execute” button in Figure A.4, this program will execute the function selected in the "Options” input. The two options available are "generate peak period factors” and “Calculate MADT.” Each function is explained as follows. 
(1) Generate peak period factors

The program will first define the peak periods for different road classes and day types based on the current ATR file. A pop-up window will show the definitions of peak periods based on the current ATR file, providing users a chance to modify the definitions (Figure A.8). The final results of estimated peak period factors are stored in a database named “peakfac.dbf.” An example output file of peak period factors is shown in Figure A.9. The total growth factors are shown only in the first four rows of Figure A.9, because their values depend only on road class and not on day type.

The ROADCLASS values of 0 through 3 in Figure A.9 represent "rural interstate highway”, "rural other highway”, “urban interstate highway” and "urban other highway.” The DAYTYPE values of 0 through 2 represent “weekday”, “weekend” and the special day specified by the user. The special day in Figure A.9 is the second Sunday in May. AMPEAK, PMPEAK and OFFPEAK represent the peak period factors for AM-peak, PM-peak and off-peak periods. These values are the adjusted peak period factors, which combine peak period factors and directionality.

AMBEGIN, AMEND, PMBEGIN and PMEND represent the beginning and end of AM-peak and PM-peak periods. TGROWTH represents the total growth factors, which are the growth factors from the base year of ADTs to the year of departure. REMARK provides definitions of the day types.

\section{(2) Calculate MADT}

The program will process one ATR file at a time and calculate the MADT for each ATR station. The resulting MADTs are stored in a file with the name of the ATR file and “ADT” appended to it. For example, the results of MADTs for the 
ATR file "maytr98.xls" are stored in a file named "maytr98ADT.xls." An example output file is shown in Figure A.10. The "Station" represents the ATR station ID. "RoadClass" and "DayType" have the same definitions as in the file of peak period factors (Figure A.9). MADT represents the monthly average daily traffic at the station for the specific road class and day type.

\section{A.2 TransCAD GISDK Interface}

The framework of the TransCAD GISDK interface is shown in Figure A.11. There are two groups who need to use the TransCAD GISDK interface: the modeling group and the project scheduling group. By entering the TransCAD, a pop-up window will show for choosing an application (Figure A.12). By selecting the "HTIS" application, the TransCAD GISDK interface will show the main menu (Figure A.13), which allows the user to select the proper user group. The user will be guided to the "modeling menu" and the user of the project scheduling group to the "project scheduling" menu. The functions provided in the modeling and project scheduling subsystems are listed as follows.

(1) The functions for the modeling subsystem provided in the TransCAD GISDK interface in the modeling subsystem include:

- Link volume forecasting;

- Delay verification;

- Delay database generation and conversion.

(2) The functions provided for the project scheduling subsystem include:

- Maintaining schedule database;

- Evaluating current scheduling strategy. 


\section{A.2.1 Modeling subsystem}

The three functions provided in the modeling subsystem - link volume forecasting, delay verification and delay database generation - are sequential processes. The link volume forecasting needs to be processed first, the delay verification second, and the delay database generation last.

\section{A.2.1.1 Link volume forecasting}

The modeling input menu (Figure A.14) will appear if the option "Modeling” is chosen in the main menu.

Step 1: Inputs for link volume forecasting

Users may provide different combinations of input files to perform two different sets of link volume forecasting analyses:

(1) Inputs for the "no information” link volume forecasting

- A map file in TransCAD map format (*.map) (Figure A.15);

- $\quad$ A peak factor file in dBaseIV format (*.dbf) (Figure A.9);

- A construction schedule file in dBaseIV format (*.dbf) (see Step 2 in Section

$$
\text { A.2.2); }
$$

- An existing hourly volume file in dBaseIV format (*.dbf) (Figure A.16) or a file name for storing hourly volumes.

(2) Inputs for both the "no information" and the "complete information" link volume forecasting

- A map file in TransCAD map format (*.map);

- A peak factor file in dBaseIV format (*.dbf); 
- A construction schedule file in dBaseIV format (*.dbf);

- An existing hourly volume file in dBaseIV format (*.dbf) or a file name for storing hourly volumes.

- An O-D matrix file in TransCAD matrix format (*.mtx) (Figure A.17).

Step 2: Execute the link volume forecasting

The forecasting process will be executed automatically after the input files are given. No manual operations are required. Users should be aware that the whole process of the "complete information" volume forecasting can take a long time depending on the number of day types and the number of combinations of different construction projects active at the same time. The execution time for a single day type and a set of construction projects active at the same time is about 40 to 50 minutes. If only a preliminary estimate of traffic conditions is needed, the "no information" link volume forecast can provide some useful trends. However, if users need to build a delay database, the "complete information” link volume forecasting is required.

Step 3: Outputs from link volume forecasting

(1) Outputs from "no information” volume forecasting

The outputs from "no information" volume forecasting are the "no information" link volumes, which are stored in the file of hourly volumes. The fields stored in the file are named as the combination of day type abbreviation, a serial number for special day types, period abbreviation and "VOL". The day type abbreviations for weekdays, weekends and special days are "WD”, "WE” and "SP." The day type of special days is distinguished by a serial number starting from 1 to the maximum allowable special day types of 10 . The period abbreviations for AM-peak, PM-peak 
and off-peak periods are "AM", "PM" and "OFF." For example, the field name of the "no information" hourly volume during AM-peak period for a typical weekday is "WDAMVOL", and the field name of the "no information" hourly volumes during off-peak period for the first special day is "SP1OFFVOL."

(2) Outputs from "complete information” volume forecasting

The outputs from "complete information" volume forecasting are the "complete information" link volumes. "Complete information" volume forecasting involves a combination of transportation planning analyses, such as O-D estimation and traffic assignment. The number of applications of O-D estimation and traffic assignment that need to be repeated are explained in Section 6.1. Both the O-D estimation and the traffic assignment processes will generate intermediate outputs before the final outputs - the "complete information" link volumes - are generated. The intermediate outputs for O-D estimation and traffic assignment, and the final outputs for the “complete information” volume forecasting, are explained as follows.

- Intermediate outputs for O-D estimation

Several intermediate files will be generated from each application of O-D estimation and traffic assignment when "complete information" link volume forecasting is performed. Each application of O-D estimation will generate two intermediate outputs.

(a) An estimated O-D table in TransCAD matrix format (*.mtx) for use by the traffic assignment process, and

(b) A binary file (*.bin) of estimated link volumes for comparison with the “observed link volumes" and for use as one of the inputs for O-D estimation. 
The "observed link volumes" are those found in the "no information" link volume forecast based on the assumption that the demand of each O-D pair before construction and during construction are the same. (See Section 5.3 for detailed explanations.)

To distinguish the outputs of each application of O-D estimation, both the estimated O-D table and estimated link volumes are named as the combination of “OD”, day type abbreviation and period abbreviation. The day type abbreviations for weekdays and special days are "WD”, and "SP1" through "SP10.” The period abbreviations for AM-peak, PM-peak and off-peak periods are "AM”, "PM”, and "OFF." For example, when generating the O-D table for the "no information" link volumes during AM-peak period in a typical weekday, the day type code for weekdays is "WD" and the period abbreviation is “AM." The estimated O-D table is named "ODWDAM.mtx" and the binary file of the estimated link volumes is named “ODWDAM.bin.”

- Intermediate outputs for traffic assignment

Each application of traffic assignment will generate an intermediate binary file (*.bin) of the "complete information" hourly volumes for all links. The binary file of the "complete information" hourly volumes is named in a different way from the output files for O-D estimation, because of the limitation of TransCAD in dealing with long file names (maximum of 8 characters). The name of the binary file of the "complete information" hourly volumes is as the combination of "TA", day type code, period code and construction starting or ending date. The day type codes are 0 for weekdays, and 2 through 12 for special days. The period codes for AM-peak, 
PM-peak and off-peak periods are 0, 1 and 2. The construction starting or ending date is the combination of month and date. For the example, the binary file of the “complete information” hourly volumes during AM-peak period (0) for a typical weekday (0) between May 2 (0502) and the next time when there is a construction project starting or ending is named "TA000502.bin.”

- Final outputs for "complete information” volume forecasting

The final outputs, the "complete information” hourly volumes from traffic assignment, will be copied to the file of hourly volumes with the field names similar to the ones with the "no information" link volumes, using the combination of "LB", day type abbreviation, period abbreviation, "VOL", and time span code. The "LB" represents the lower bound of workzone link volumes, which are the "complete information” link volumes. The definitions for day type abbreviation, period abbreviation and "VOL" are the same as the definitions for the "no information" link volumes. The time span code is a serial number to distinguish different time spans, each of which represents the time span between when a project starts or ends and the next time a project starts or ends, or a special day occurs (Figure 6.5). The first time span is always for the period between the first day of the specific month and the first time a project starts or ends in the month. The following time spans are defined as between the time when a project starts or ends and the next time a project starts or ends. The final time span is between the last time when a project starts or ends before the end of the month and the end of the month. For example, there is a special day in the first time span of a specific month, which is between the first day of the month and the time the first project starts or ends. The field name of 
the “complete information” hourly volumes during AM-peak period for a typical weekday in the first time span is "LBWDAMVOL1", and the field name of the hourly volumes during the off-peak period for the special day in the first time span is "LBSP1OFFVOL1."

\section{A.2.1.2 Delay verifications}

The main menu for verifying possible delays according to the forecasted volumes will appear after the volume forecasting process (Figure A.18).

Step 1: Inputs for delay verification

The inputs required are the day type, time of day and a selected link for evaluation.

They are addressed as follows.

(1) Day type

The day type options are read from the peak period factor file that is given in the modeling input menu (Figure A.18). The day type, together with the time of day in the following input items, determine which field of hourly volumes in the file of hourly volumes to retrieve for verifying delays.

(2) Departure time

The "Departure time” is to specify a departure time for delay estimation. The "Departure time" is in one-hour increments. The delays during the one-hour period are estimated based on the corresponding forecasted hourly volumes. 
(3) A selected link

The link for evaluation is selected by clicking on the "Select a link" button on the menu first. The pointer of the mouse will become a cross when the mouse is moved on the map. The user may select a link by clicking on it.

Step 2: Execution and the outputs for delay verification

When a link on the map is selected, the color of the link is changed to red and a pop-out message box with information of estimated delay is shown. The upper bound delay is shown if only the "no information" link volumes are forecasted (Figure A.19). The adjusted delay is shown if both the "complete information" and the "no information" link volumes are available (Figure A.20). Users can continue the delay evaluation without having to click on the "Select a link" button again if the pointer of the mouse remains a cross while moving the mouse over the map. When the link delays are not acceptable, users may either adjust the peak period factors in the peak factor file (Figure A.9) or regenerate the peak factor file by changing the "Annual Growth Factors" in Figure A.4 or the definitions of peak periods in Figure A.8.

\section{A.2.1.3 Delay database generation and conversion}

Step 1: Delay database generation

If both the "no information" link volumes and the "no information" link volumes are available and the accuracy of the estimated delays is verified by the user, the delay database can be generated by clicking on the "Generate delay database" button on the main menu (Figure A.18). The file of the delay database can be saved in dBaseIV format (*.dbf). The name of the file can be input in the pop-out dialog box (Figure 
A.21). After the delay database is generated, the tasks in the TransCAD GISDK interface are finished.

Step 2: Delay database conversion

The delay database needs to be converted into Microsoft Access format before the public can access it. The file conversion process under Microsoft Access 2000 begins by creating a new empty database. Select the option "Blank Access database” under the group "Create a new database using” (Figure A.22) or by selecting "File” and then “New” from the menu bar. By selecting “File”, "Get External Data” and then "Import” from the menu bar, a pop-up dialog box will appear, asking which file to import (Figure A.23). Users need to change the "Files of type" to "dBaseIV (*.dbf) and then select the file name of the delay database from the file lists. After importing the data, the delay database in Microsoft Access format can be uploaded to the web server for public access. An example delay database in Microsoft Access format is shown in Figure A.24.

\section{A.2.2 Project scheduling menu}

The functions provided for project scheduling personnel are to maintain the project schedule database and to evaluate current scheduling strategy. Maintaining the schedule database involves adding, updating and/or deleting construction projects including the start date, end date, number of lane closures and a brief description of the project content. Evaluating current scheduling strategy uses two performance indices: “Link delay” and “Total delay.” "Link delay” is used to check a specific link that is under construction, while “Total delay" is for checking the system performance for the current scheduling 
strategy. Because the purpose of evaluating current scheduling strategy is to calculate performance indices based on a schedule database, this function is embedded in schedule management. (See Figure A.11.)

Step 1: Inputs to the project scheduling subsystem

If the option "Project Scheduling” is chosen in the "User Group” of the HTIS main menu (Figure A.12), the project scheduling management input menu (Figure A.25) will appear. Users may provide different combinations of input files to perform two different combinations of functions.

(1) The inputs required for scheduling management include:

- A network file in TransCAD map format (*.map);

- Either an existing construction schedule file in dBaseIV format (*.dbf) or a file name for storing construction schedules.

(2) The inputs needed for evaluating current scheduling strategy depend on the purpose for evaluation. If the purpose is to get a rough idea of the impacts on traffic, only the "no information" analysis, which produces the upper bound of link delays, is needed. If the evaluation is for considering the effects of construction during transition period when the workzone is not known to most of the travelers, both the "no information" and the "complete information" analyses are required.

- Inputs for the "no information" analysis

- A map file in TransCAD map format (*.map) (see Figure A.15);

- A peak factor file in dBaseIV format (*.dbf) (see Step 3 in Section A.1 and Figure A.9); 
- An existing construction schedule file in dBaseIV format (*.dbf) (see Step 2 in this section);

- An existing hourly volume file in dBaseIV format (*.dbf) (see Step 3 in Section A.2.1.1 and Figure A.16).

- Inputs for both the "no information" and the "complete information" analyses

- A map file in TransCAD map format (*.map);

- A peak factor file in dBaseIV format (*.dbf);

- A construction schedule file in dBaseIV format (*.dbf);

- An existing hourly volume file in dBaseIV format (*.dbf) or a file name for storing hourly volumes.

- An O-D matrix file in TransCAD matrix format (*.mtx) (Figure A.17).

Step 2: Schedule management

The main menu for project scheduling management will appear after the required inputs are provided (Figure A.26). This menu is a simple database management system with the function of connecting the database to the geographic attributes. Each record in the scheduling database contains fields for the project beginning date, end date, workzone capacity, a brief description of the project, link ID as noted in TransCAD, and the coordinates of two end nodes for the link. The link ID and node coordinates are automatically linked to the inputs above.

(1) “Add”, "Save edited”, and “Delete” functions

Users must select a link by clicking on the "Select a link" button on the menu before adding, editing and deleting any records. The function of "Select a link" here is the same as it was in the delay verification menu in the modeling subsystem. Once 
the button is clicked, the pointer of the mouse will change into a cross when the mouse is moved over the map. Users may select a link on which to apply construction constraints, by clicking on the button "Add", "Save edited" or "Delete" to add, edit or delete a record in the construction schedule database. These three buttons are disabled if no link is selected, because no geographic attributes can be linked to the manual inputs. Each time a record is added, edited or deleted, the modifications are directly saved in the database. Therefore, there is no need to save the database.

(2) “Clear” function

The function of "Clear" in Figure A.26 is to empty the current manual inputs to allow more convenient entry of a different set of inputs.

(3) "Show all" function

The function of "Show all" is to show the existing records in the scheduling database. When there are records in the scheduling database, both the records and the locations will be displayed in a table and on the map when the "Show all" button is clicked. The records in a table are shown in a new window and the locations on the map are shown in thick dark green lines (Figure A.27). This function is disabled when there is no record in the scheduling database.

Step 3: Evaluation of current scheduling strategy

The function of evaluating current scheduling strategy provides two performance indices: "Link delay" and "Evaluate schedule.” These two functions are embedded in the schedule management menu (Figure A.26). 
(1) "Link delay" function

The function of "Link delay" is to display the estimated delay on any selected link, given to the current scheduling strategy. When this function is chosen, the project scheduling subsystem is linked to the "no information" and the "complete information" link volume forecasting in the modeling subsystem. After finishing the "no information" and the "complete information” link volume forecasting, the users in the project scheduling group will be led to the delay verification menu in the modeling subsystem (Figure A.18). It should be noted that this function may require a long execution time depending on the number of day types and the number of combinations of different construction projects active at the same time. The execution time for a single day type and a set of construction project active at the same time is about 40 to 50 minutes. The user should use this function only if all schedules have been updated. If not all of the inputs for evaluating current scheduling strategy are provided in the input menu for the project scheduling subsystem (Figure A.25), this function is disabled.

(2) “Evaluate schedule” function

Using the function "Evaluate schedule", the total delay on all workzone links for selected periods is calculated. The user is led to the scheduling evaluation menu (Figure A.28). This function may also take a long time to execute. As with the execution time for the function "Link delay", the total execution time depends on the number of day types and the number of combinations of different construction projects active at the same time. The execution time for a single day type and a set of construction project active at the same time is about 40 to 50 minutes. The user 
should use it only if all schedules have been updated. If not all of the inputs for evaluating current scheduling strategy are provided in the input menu for the project scheduling subsystem (Figure A.25), this function is disabled.

The "Evaluate schedule" function is completed with the following two processes.

- Determine evaluation period

Users may determine the evaluation period for total delay in two ways.

- Click the "Select a link" button in Figure A.28 and then click on a desired link that belongs to a construction project. The total system delay during the time when the specific construction project is in progress within the month specified in the peak factor file will be calculated.

- By entering the start date and the end date as evaluation period: The total delay during the evaluation period will be calculated.

- Calculate expected delay for all links

After defining the evaluation period, users are led to the modeling subsystem to calculate the expected delays of all links under construction during the specified evaluation period. The sum of the product of the expected delays and volumes for each workzone link is defined as the total statewide delay for the specific period of time. The total delay for the specific period of time is then passed back to the project scheduling subsystem and shown in a message box (Figure A.29).

The calculation of total delay may be done again in two ways.

- Change the evaluation period in the scheduling evaluation menu (Figure A.28) and calculate the total delay based on the existing scheduling strategy; 
- Press the "Back" button to the schedule management menu (Figure A.26), change scheduling strategy, and then press the "Evaluate schedule" button to return to the schedule evaluation menu for calculating total delay based on the updated scheduling strategy. Schedule strategies can be changed by inputting different project beginning dates, project end dates, or number of lanes closed that is reflected by entering different workzone capacities. The scheduling evaluation can be repeated until the project schedules have been improved as much as possible, according to the judgment of the project scheduler.

\section{A.3 Web Access Interface}

The framework of the web access interface is shown in Figure A.30. The web access subsystem is an interactive interface for highway travelers to get information about delays at workzones via web browsers. Travelers may repeatedly query the subsystem by changing the departure time.

Step 1: Enter web access interface

Currently, the web access subsystem is uploaded to a temporary web server that supports ASP scripting. The temporary web address for the web access subsystem is http://domaindlx.com/shuling/test/htis.htm. The official web address for the subsystem will be determined by INDOT.

Step 2: Inputs for the web access interface

The homepage of the web access subsystem is shown in Figure A.31. Travelers may follow the instructions on the page to obtain delay information for a specified time. Two inputs are required for the web access subsystem: 
(1) Departure date

The departure date can be any date between current date and the end of the next month. More information can be provided if the construction schedules can be set more than one month in advance. See Section 6.3 for more explanations.

(2) Departure time

The departure time is in hourly periods. If a traveler plans to departure at 7:30AM, the departure time of 7:00AM 8:00AM should be selected.

Step 3: Outputs for the web access interface

After the two required inputs are given, travelers may click on the "Submit" button to view estimated delays due to workzones at the specific time. Figure A.32 shows the response page of the query results. Because the delay database has not been established, the ATR station database is used for illustration purpose. The search criteria for the ATR station database is "County ID” in contrast to the search criteria “Departure Date” and "Departure Hour” for the delay database. Each icon in Figure A.32 is supposed to represent a construction project workzone expected to be in place during the time specified by travelers.

The web access subsystem provides two ways to view delay information for each project:

(1) By moving the mouse on top of a workzone icon

A “tip box" will appear, showing the estimated delay for that workzone. For example, the tip box in Figure A.32 shows "Site 2 on US 52: Delay = 46 minutes" when the mouse is moved on top of the workzone Site 2. 
(2) By clicking on the workzone icon

The detailed information about the corresponding project will appear in the textbox to the right of the map. For example, the detailed information in Figure A.32 shows "Site 2 on US 52: Delay $=46$ minutes. This project will be finished by 9 December 2000.” when the icon of the workzone Site 2 is clicked. Each time an icon is clicked, the contents of the textbox is automatically changed.

Travelers may input a different departure date and time right under "New search" in the same page without having to go back to the homepage (Figure A.32). Because the processing time for each query is only a few seconds, travelers can query many times in a few minutes and decide on the best departure time for their trips. 


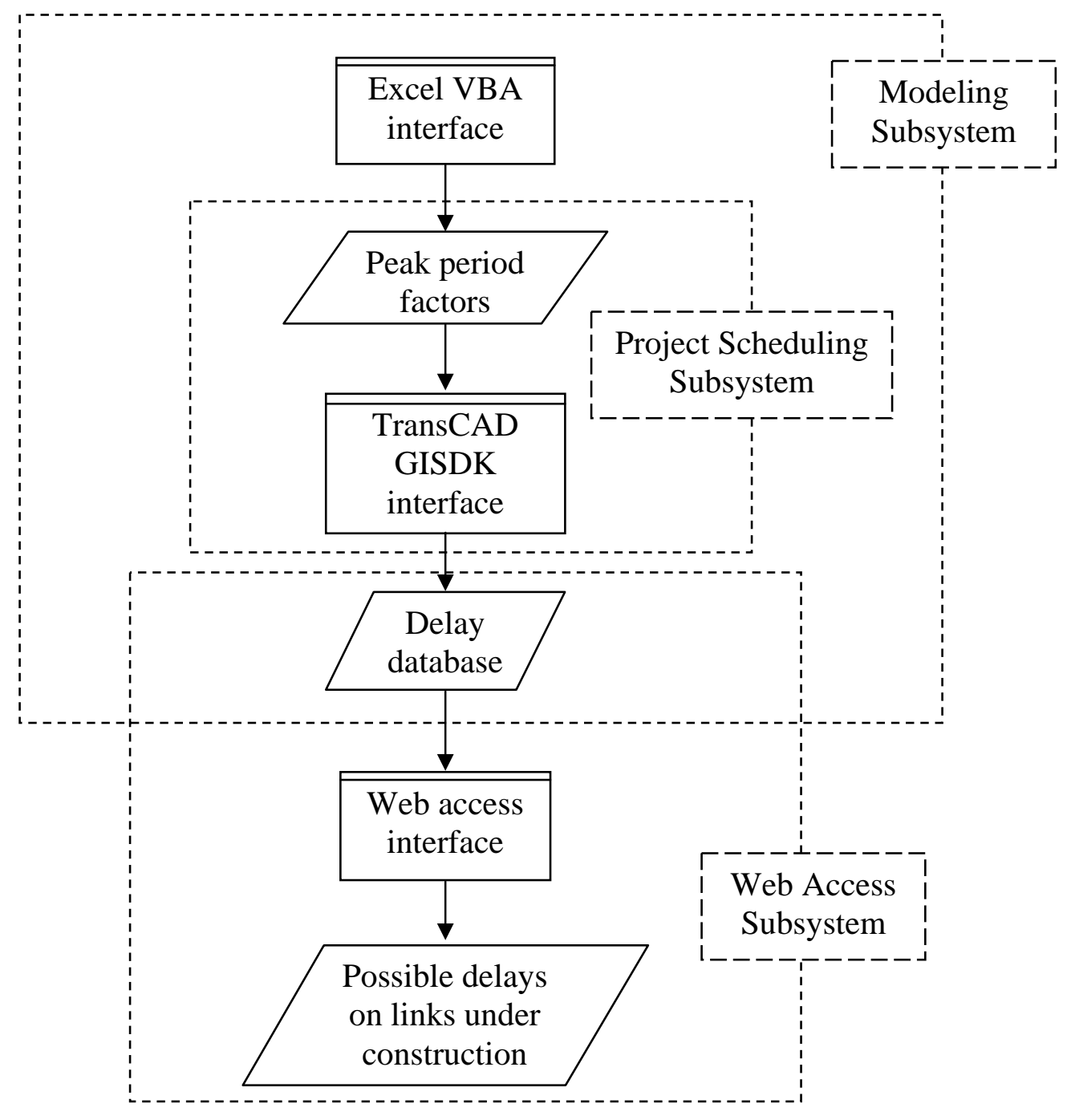

Figure A.1 Relationship of HTIS users’ interfaces 


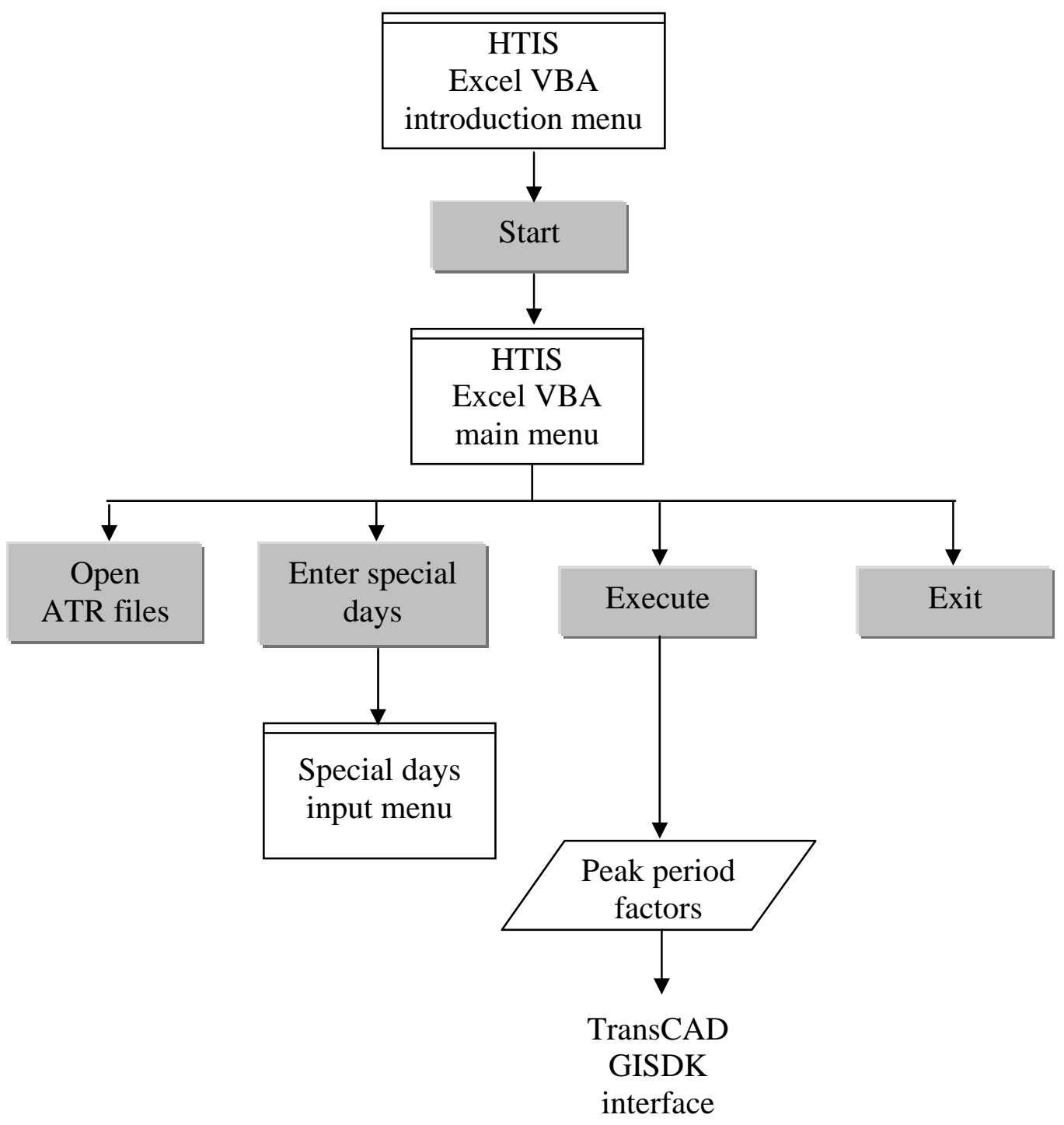

Figure A.2 Framework of the Excel VBA interface 


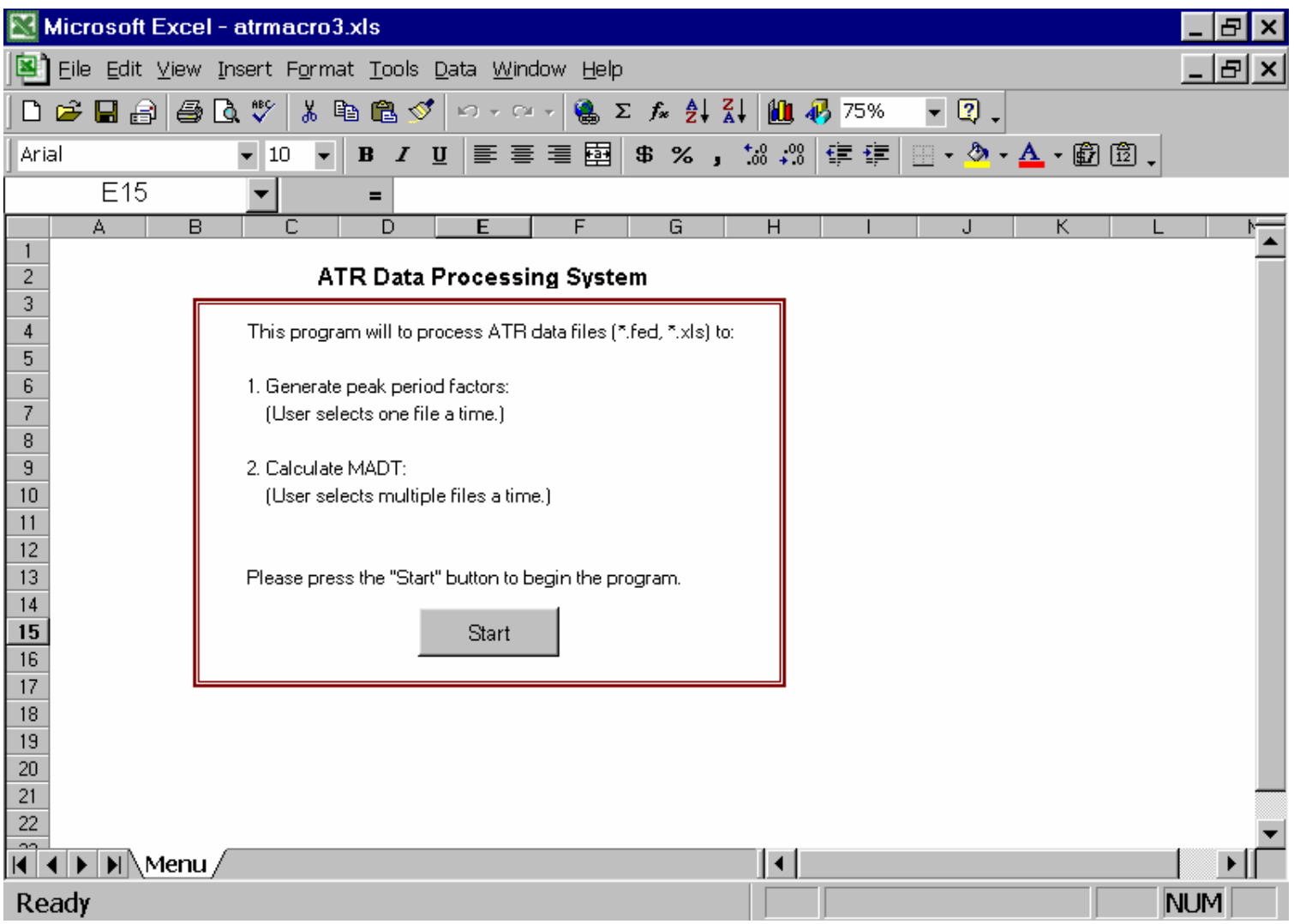

Figure A.3 Excel VBA introduction menu for the modeling subsystem 


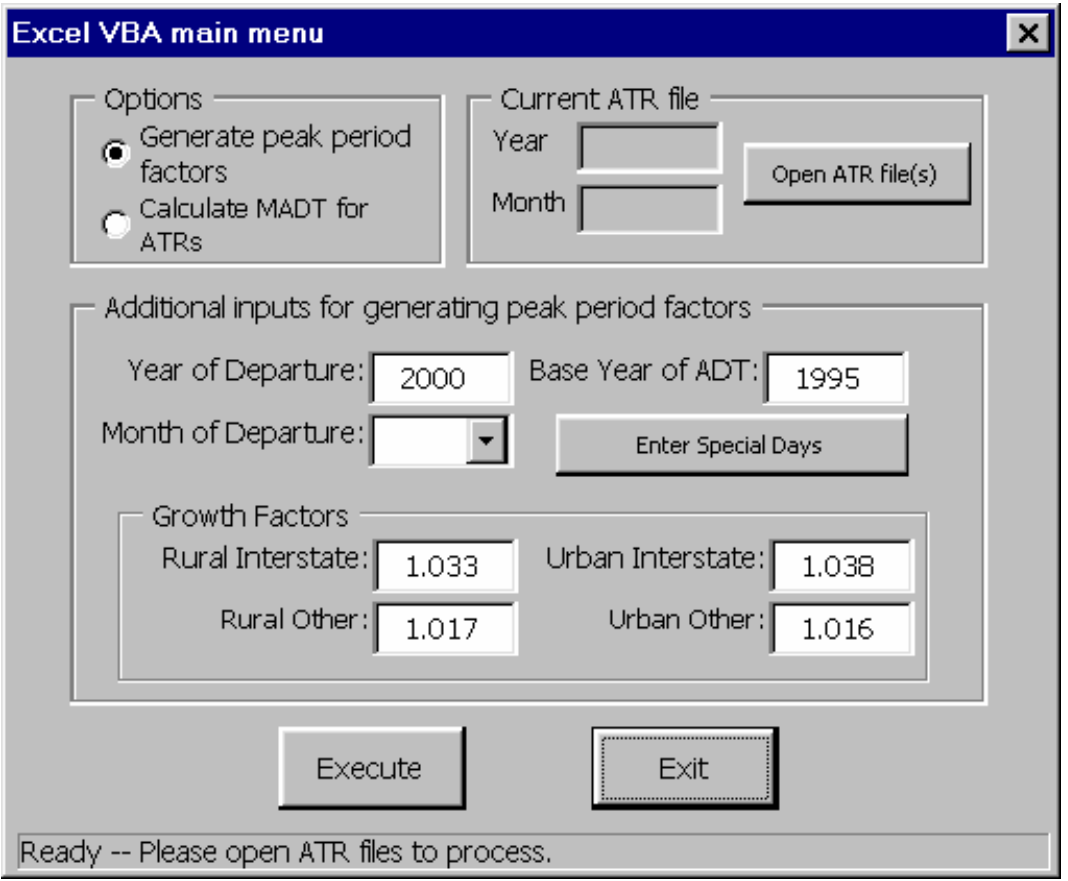

Figure A.4 Excel VBA main menu for modeling subsystem 


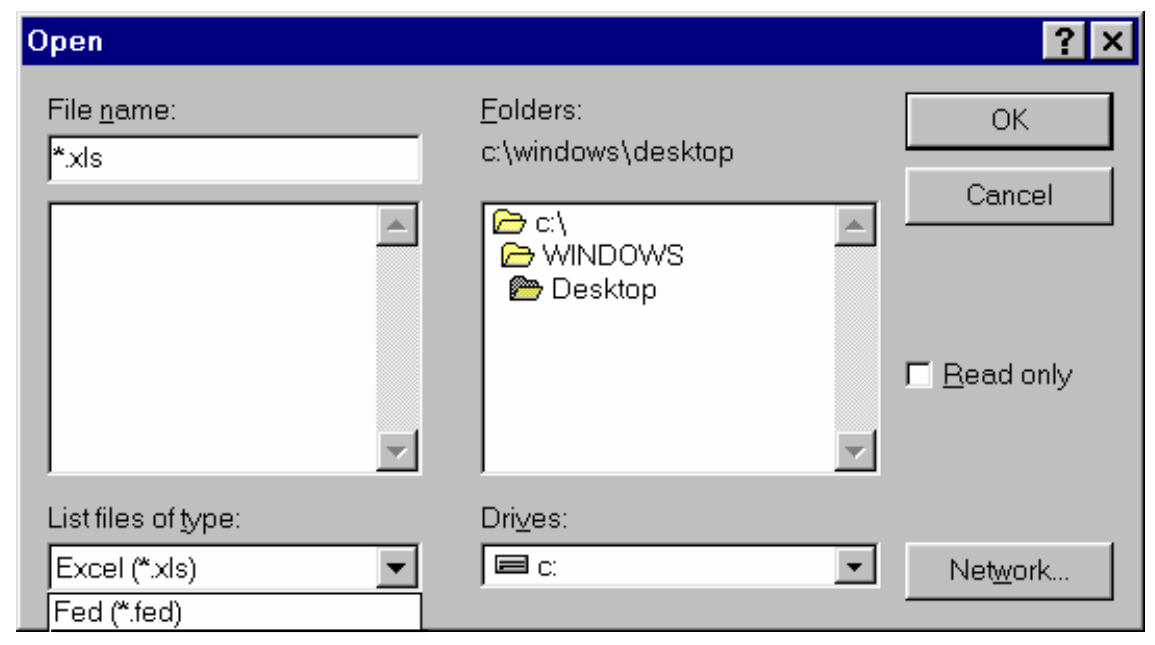

Figure A.5 Open ATR files from the Excel VBA main menu 


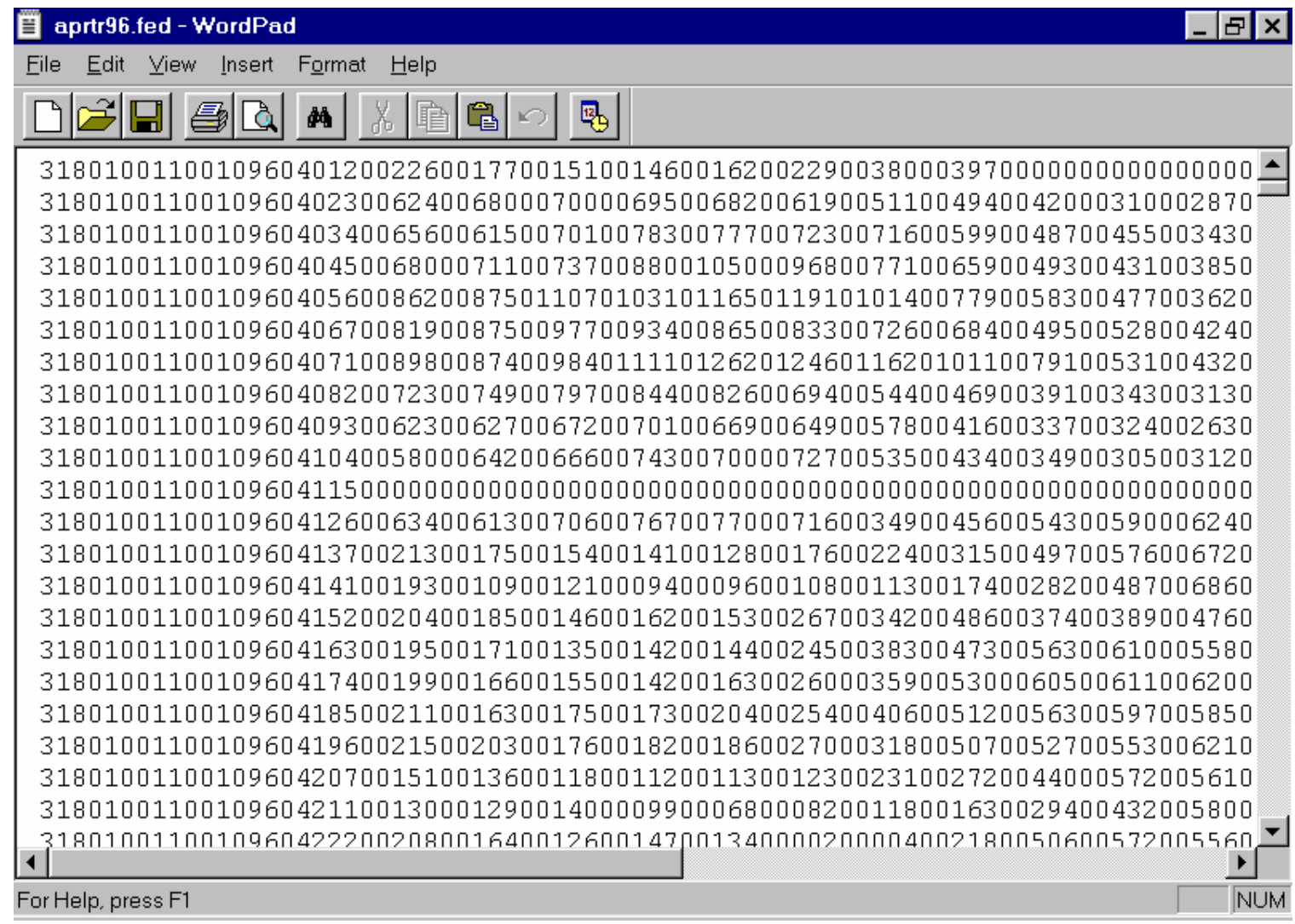

Figure A.6 An example ATR file in federal format (*.fed) 


\section{Add Special Days for May}

\begin{tabular}{|c|c|c|c|c|c|c|c|c|}
\hline 1. By date: & 13 & $\nabla$ & $C$ By day of week: & & - & & - & C None \\
\hline 2. $\bigcirc$ By date: & & $\nabla$ & (C By day of week: & 2nd & - & Sun & - & $C$ None \\
\hline 3. By date: & & $\nabla$ & $C$ By day of week: & & - & & 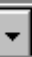 & 6 None \\
\hline 4. $\bigcirc$ By date: & & $\nabla$ & By day of week: & & - & & - & $\sigma$ None \\
\hline 5. By date: & & $\nabla$ & $C$ By day of week: & & 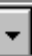 & & - & 6 None \\
\hline 6. $\bigcirc$ By date: & & $\nabla$ & $\checkmark$ By day of week: & & - & & 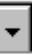 & 6 None \\
\hline 7. $C$ By date: & & $\checkmark$ & $C$ By day of week: & & - & & - & 6 None \\
\hline 8. By date: & & $\nabla$ & By day of week: & & $\checkmark$ & & - & 6 None \\
\hline 9. By date: & & $\nabla$ & By day of week: & & - & & - & 6 None \\
\hline 10. $\bigcirc$ By date: & & $\nabla$ & $C$ By day of week: & & - & & - & 6 None \\
\hline
\end{tabular}

\begin{tabular}{|c|}
\hline CK \\
\hline
\end{tabular}

Figure A.7 Special days input menu in the Excel VBA interface 


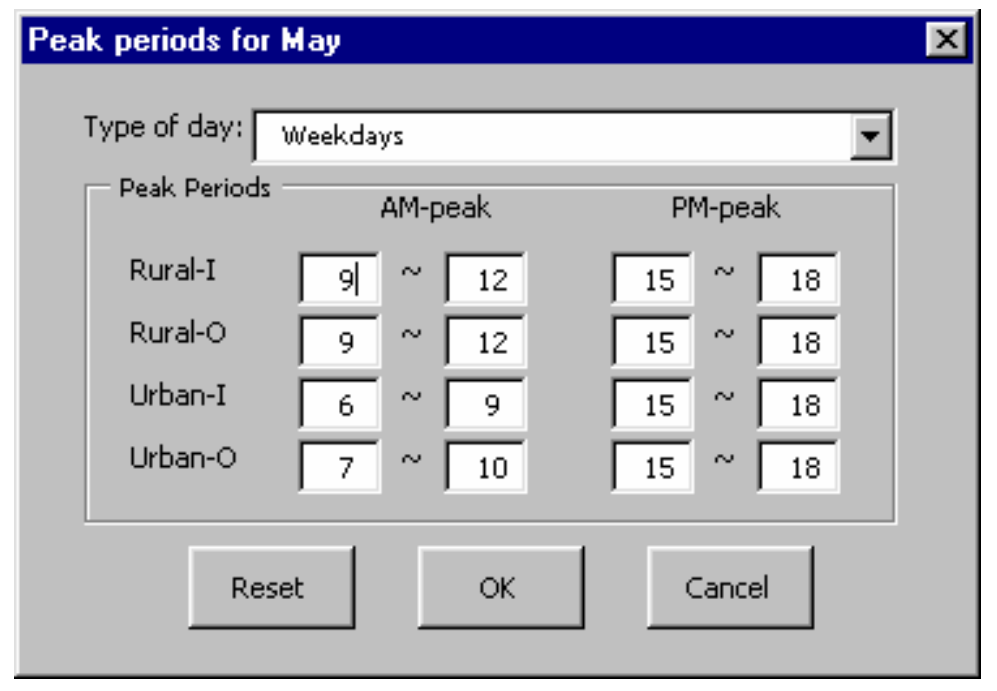

Figure A.8 Definitions of peak periods for different day types and road classes 


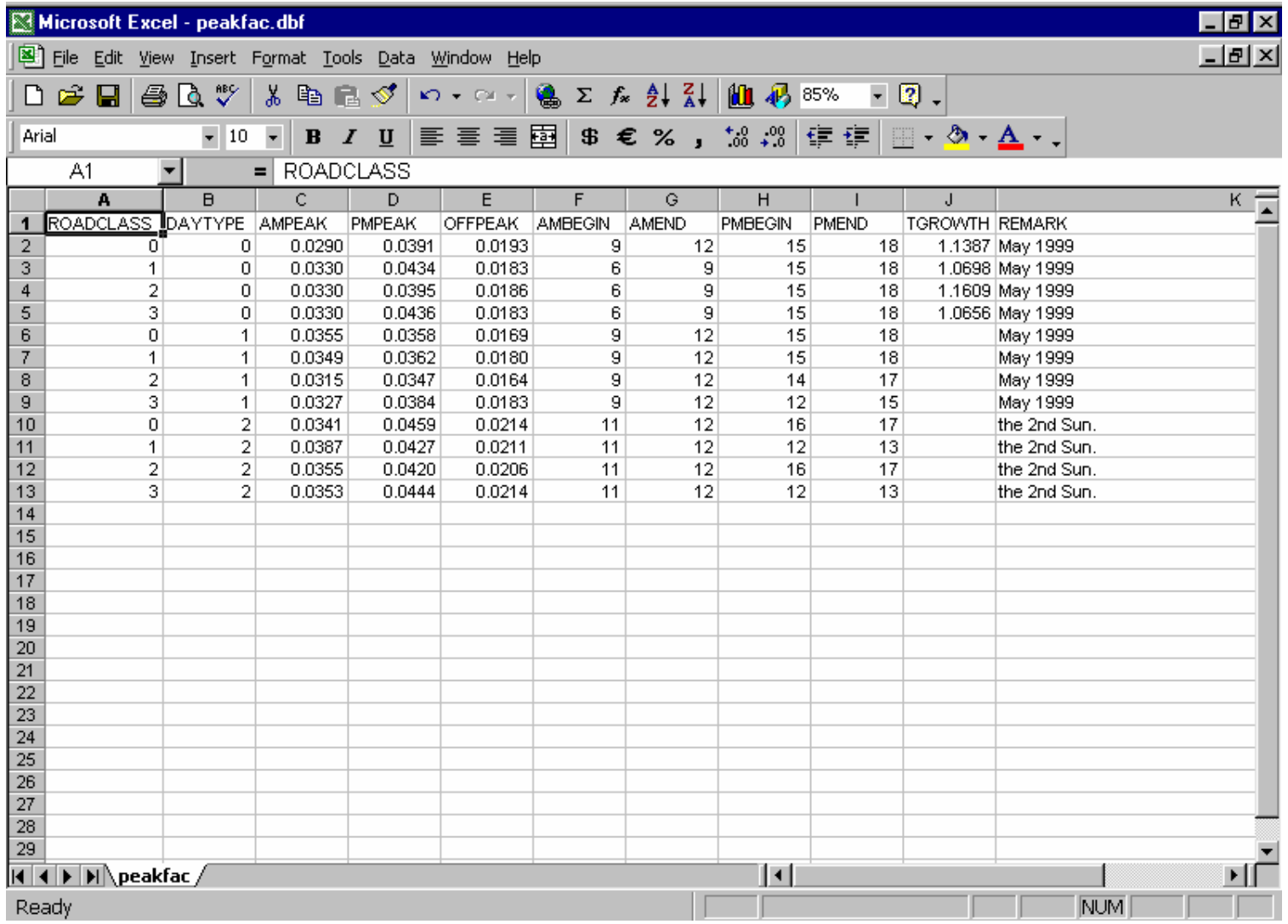

Figure A.9 The output file of the peak period factors from the Excel VBA interface 


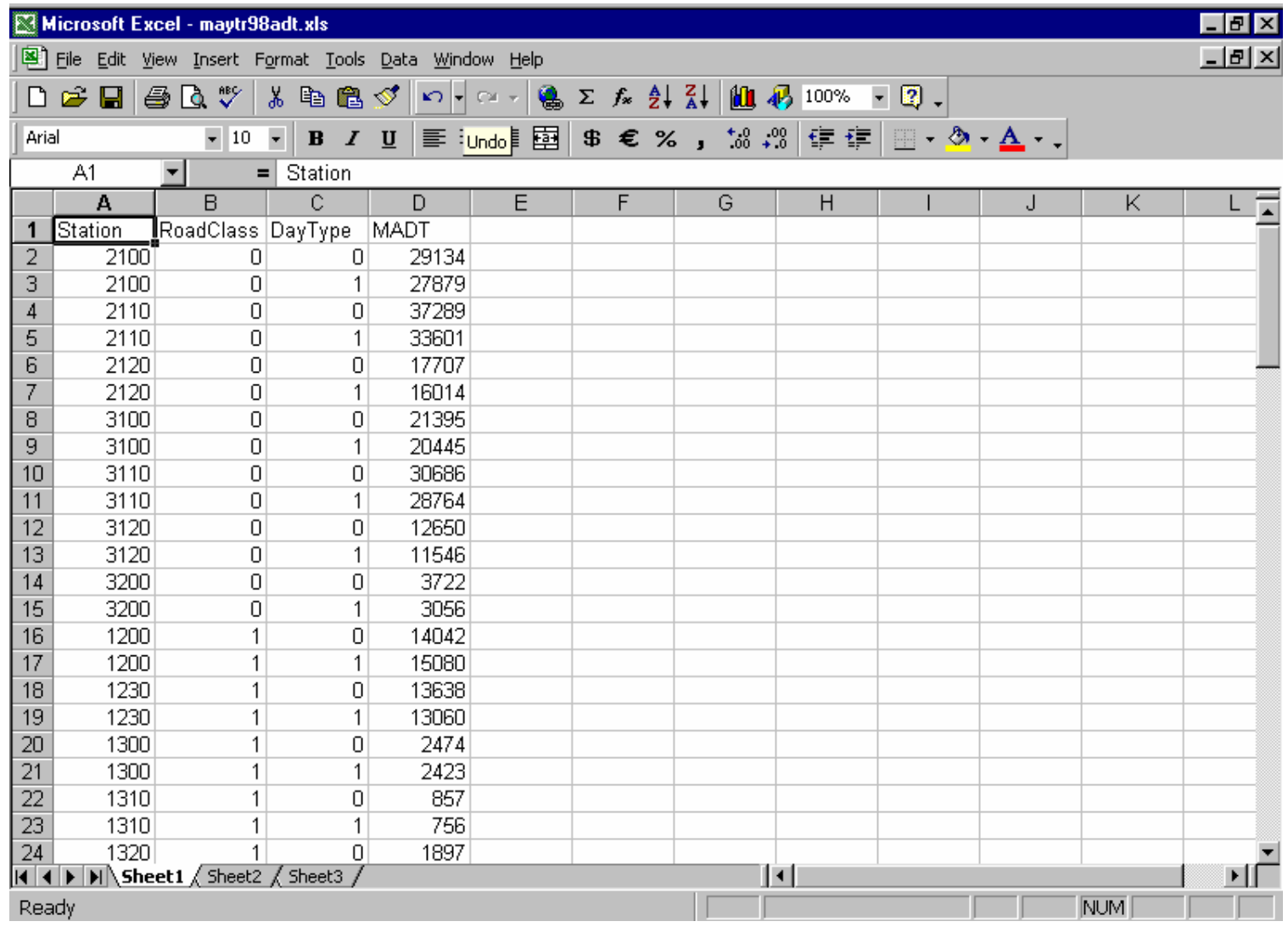

Figure A.10 The output file of MADTs from the Excel VBA interface 


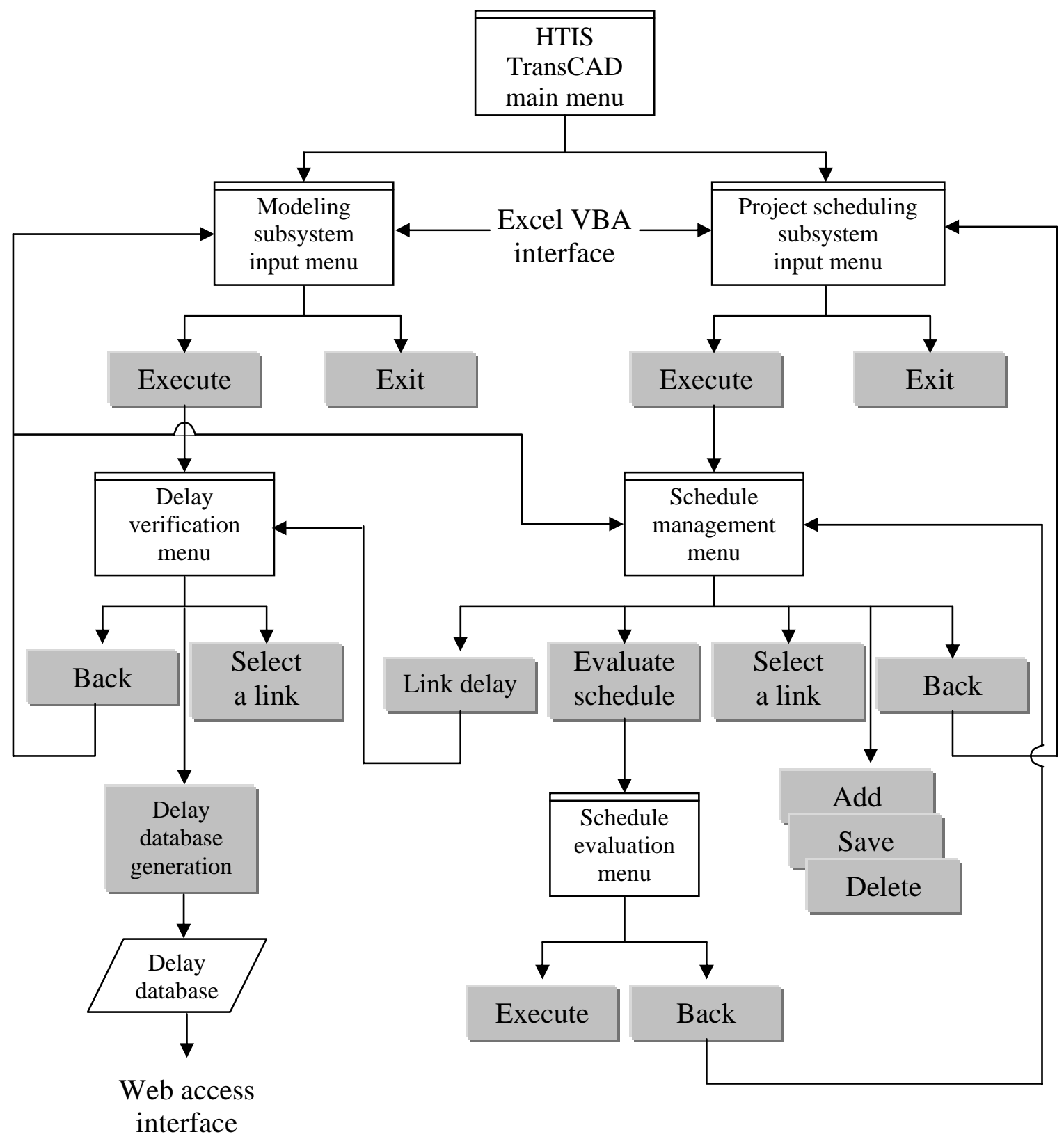

Figure A.11 Framework of the TransCAD GISDK interface 


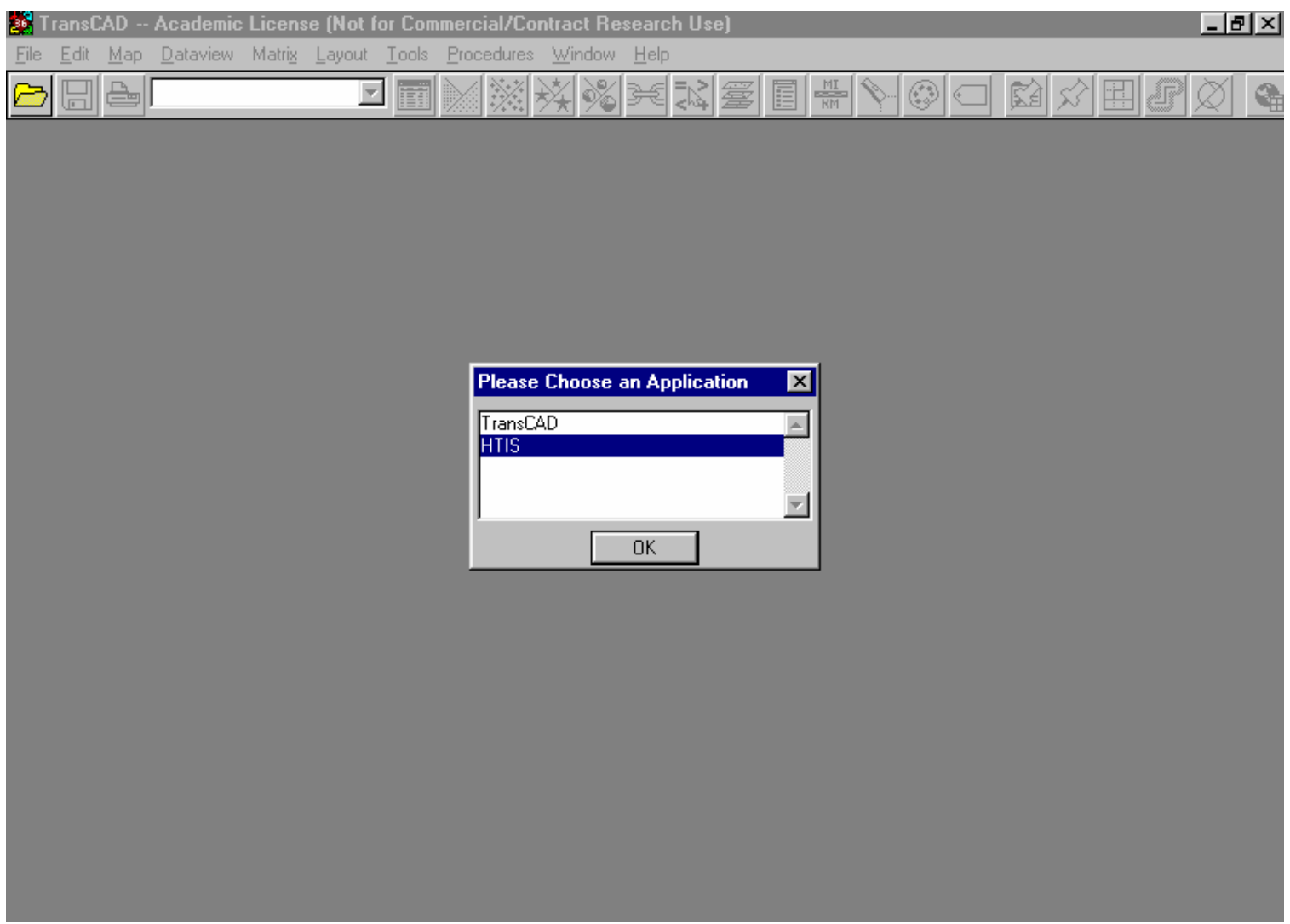

Figure A.12 Screen capture for entering the TransCAD GISDK interface for HTIS 


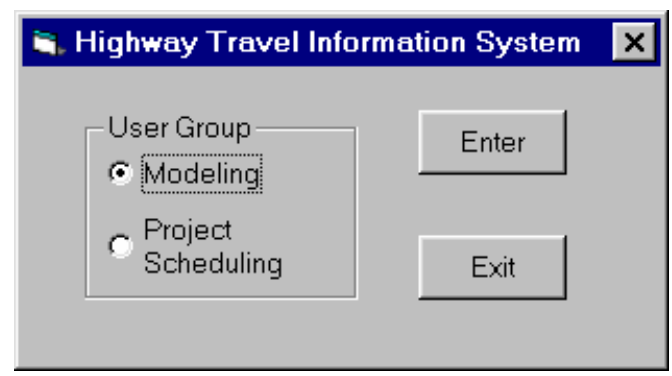

Figure A.13 Main menu of the TransCAD GISDK interface 


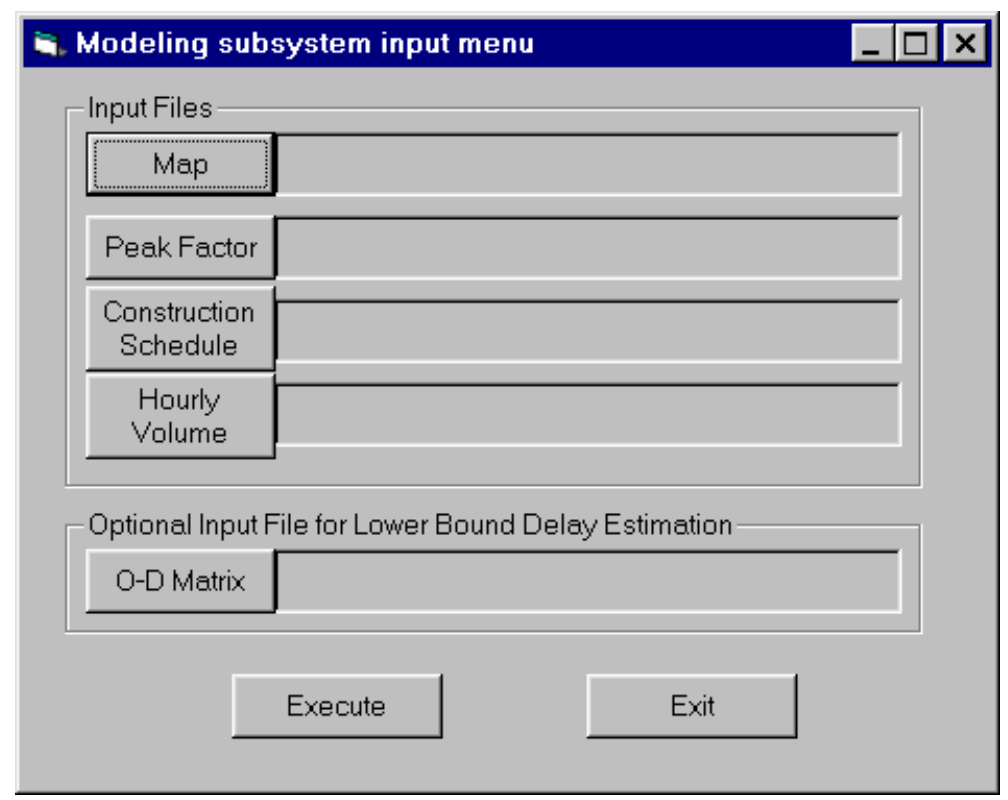

Figure A.14 Input menu for TransCAD GISDK interface in modeling subsystem 


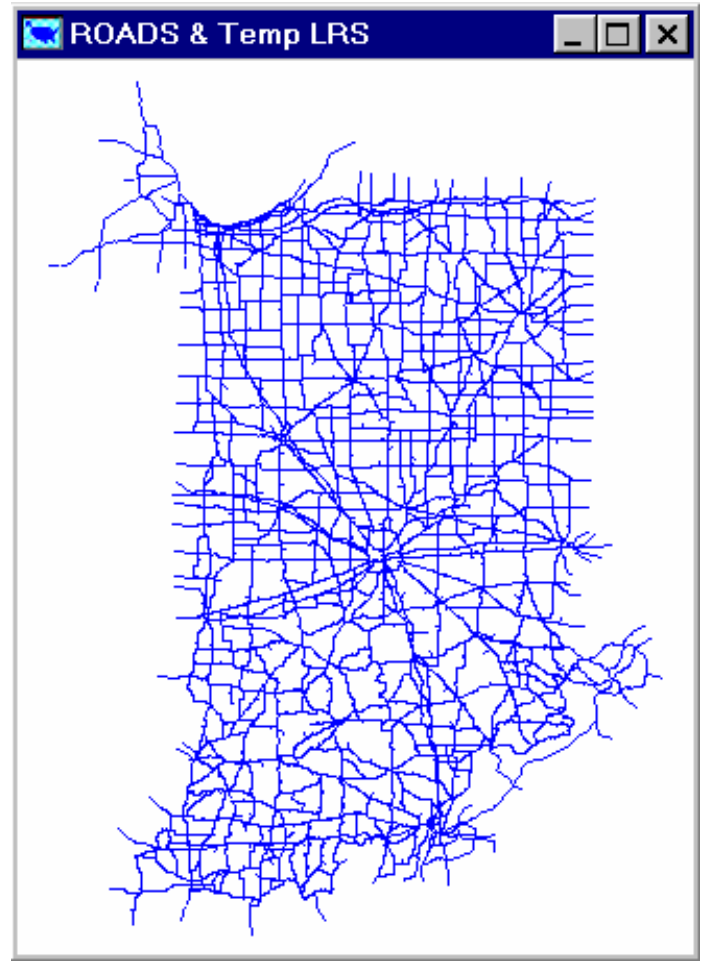

Figure A.15 A map file in TrnsCAD map format (*.map) 


\begin{tabular}{|c|c|c|c|c|c|c|c|c|c|c|c|}
\hline है? & ransCAD - & - Academic Li & icense [Not f & or Commerc & Contract & earch U: & בנ & & & & - \\
\hline 征 & File Édit & Map Dataview & Matrix Layc & out Iools $\mathrm{Pr}$ & cedures Wir & ow 브elp & & & & & - 回X \\
\hline 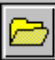 & 回鸹 & All Records & 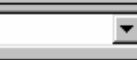 & 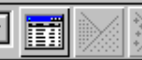 & $\left|t^{2} / *\right| 0$ & 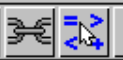 & 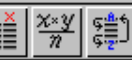 & 㗝言 & $\%$ & $\underline{E}$ & $\theta$ \\
\hline & LINKID & FHWA_FC & FROM_LAT & FROM_LON & TO_LAT & TO_LON & HRCAP & WZCAP & WDAMVO & WDPMVOL & WDOFIA \\
\hline & 19436 & 1 & 41860562 & -86615222 & 41860562 & -86615222 & 5533.00 & 5533.00 & . & -- & \\
\hline & 19475 & 11 & 41963647 & -87746992 & 41963647 & -87746992 & 6354.50 & 6354.50 & 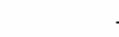 & -- & \\
\hline & 19481 & 11 & 41990766 & -87867754 & 41990766 & -87867754 & 6354.50 & 6354.50 & 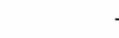 & -- & \\
\hline & 19615 & 98 & 37697157 & -87103489 & 37697157 & -87103489 & 499999.50 & 499999.50 & . & -- & \\
\hline & 19613 & 98 & 37944147 & -88125943 & 37944147 & -88125943 & 499999.50 & 499999.50 & - & -- & \\
\hline & 19465 & 11 & 41521296 & -87990430 & 41521296 & -87990430 & 6354.50 & 6354.50 & 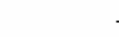 & -- & \\
\hline & 19469 & 11 & 41760206 & -87890694 & 41760206 & -87890694 & 6354.50 & 6354.50 & & -- & \\
\hline & 19467 & 11 & 41486062 & -88196539 & 41486062 & -88196539 & 6354.50 & 6354.50 & 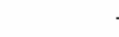 & -- & \\
\hline & 19466 & 11 & 41486062 & -88196539 & 41486062 & -88196539 & 6354.50 & 6354.50 & & -- & \\
\hline & 19589 & 98 & 41385933 & -88556974 & 41385933 & -88556974 & 499999.50 & 499999.50 & - & -- & \\
\hline & 19590 & 98 & 41237587 & -88222400 & 41237587 & -88222400 & 499999.50 & 499999.50 & & -- & \\
\hline & 19470 & 11 & 41614206 & -88162720 & 41614206 & -88162720 & 6354.50 & 6354.50 & & -- & \\
\hline & 4335 & 14 & 39844030 & -86108272 & 39844030 & -86108272 & 1183.50 & 1183.50 & 667.8 & 944.64 & 4 \\
\hline & 4357 & 11 & 39846616 & -86044208 & 39846616 & -86044208 & 7176.00 & 7176.00 & 7326.6 & 9005.66 & 411 \\
\hline & 4372 & 14 & 39845420 & -86007792 & 39845420 & -86007792 & 856.00 & 856.00 & 686.9 & 971.79 & 4: \\
\hline & 4375 & 14 & 39856493 & -85985198 & 39856493 & -85985198 & 551.00 & 551.00 & 626.8 & 886.78 & $3 t$ \\
\hline & 4429 & 6 & 40045686 & -85991029 & 40045686 & -85991029 & 591.00 & 591.00 & 421.8 & 572.50 & $2 !$ \\
\hline & 4445 & 6 & 39818871 & -87236612 & 39818871 & -87236612 & 434.50 & 434.50 & 148.4 & 201.50 & $\vdots$ \\
\hline & 4475 & 7 & 39818696 & -87104400 & 39818696 & -87104400 & 417.00 & 417.00 & 37.4 & 50.83 & : \\
\hline & 4600 & 7 & 38082545 & -86029685 & 38082545 & -86029685 & 439.50 & 439.50 & 56.9 & 77.24 & : \\
\hline & 4642 & 7 & 38241116 & -85983324 & 38241116 & -85983324 & 573.50 & 573.50 & 106.1 & 144.04 & I \\
\hline & 4648 & 1 & 38265391 & -85999853 & 38265391 & -85999853 & 3528.00 & 3528.00 & 1112.7 & 1260.37 & $7:$ \\
\hline & 4805 & 6 & 38489776 & -86003701 & 38489776 & -86003701 & 486.50 & 486.50 & 283.9 & 385.30 & 1 \\
\hline & 4870 & 7 & 38588842 & -86023578 & 38588842 & -86023578 & 394.50 & 394.50 & 29.3 & 39.82 & \\
\hline & 5013 & 7 & 39817458 & -86657007 & 39817458 & -86657007 & 417.00 & 417.00 & 33.8 & 45.93 & : \\
\hline & 5032 & 7 & 39856518 & -86637613 & 39856518 & -86637613 & 417.00 & 417.00 & 46.1 & 62.63 & $\therefore$ \\
\hline 11 & & & & & & & & & & & $\nu$ \\
\hline
\end{tabular}

Figure A.16 An existing hourly volume file in dBase IV format (*.dbf) 


\begin{tabular}{|c|c|c|c|c|c|c|c|c|}
\hline \multicolumn{7}{|c|}{ Estimated Matrix (ODME) } & \multirow{2}{*}{\begin{tabular}{|l|l|}
- & $\square$ \\
310551
\end{tabular}} & \multirow{2}{*}{$\frac{x}{4}$} \\
\hline & 310413 & 310546 & 310547 & 310548 & 310549 & 310550 & & \\
\hline 310413 & 1431.30 & 0.21 & 0.21 & 0.22 & 0.25 & 0.76 & 0.76 & \\
\hline 310546 & 0.21 & 1.16 & 1.16 & 1.16 & 1.16 & 1.16 & 1.16 & \\
\hline 310547 & 0.21 & 1.16 & 1.16 & 1.16 & 1.16 & 1.16 & 1.16 & \\
\hline 310548 & 0.21 & 1.16 & 1.16 & 1.16 & 1.16 & 1.16 & 1.16 & \\
\hline 310549 & 0.24 & 1.16 & 1.16 & 1.16 & 1.16 & 1.16 & 1.16 & \\
\hline 310550 & 0.76 & 1.29 & 1.29 & 1.29 & 1.29 & 1.29 & 1.29 & \\
\hline 310551 & 0.76 & 1.29 & 1.29 & 1.29 & 1.29 & 1.29 & 1.29 & \\
\hline 310552 & 1.65 & 1.29 & 1.29 & 1.29 & 1.29 & 1.29 & 1.29 & \\
\hline 310553 & 2.12 & 1.29 & 1.29 & 1.29 & 1.29 & 1.29 & 1.29 & \\
\hline 310554 & 0.44 & 5.26 & 5.26 & 5.26 & 5.26 & 5.26 & 5.26 & \\
\hline 310555 & 0.15 & 3.83 & 3.83 & 6.50 & 6.50 & 6.50 & 6.50 & \\
\hline 310556 & 0.30 & 1.26 & 1.26 & 2.91 & 2.91 & 2.91 & 2.91 & $\checkmark$ \\
\hline \begin{tabular}{l|l|}
1 & \\
\end{tabular} & & & & & & & $\nu$ & IIS \\
\hline
\end{tabular}

Figure A.17 An O-D matrix file in TransCAD matrix format (*.mtx) 


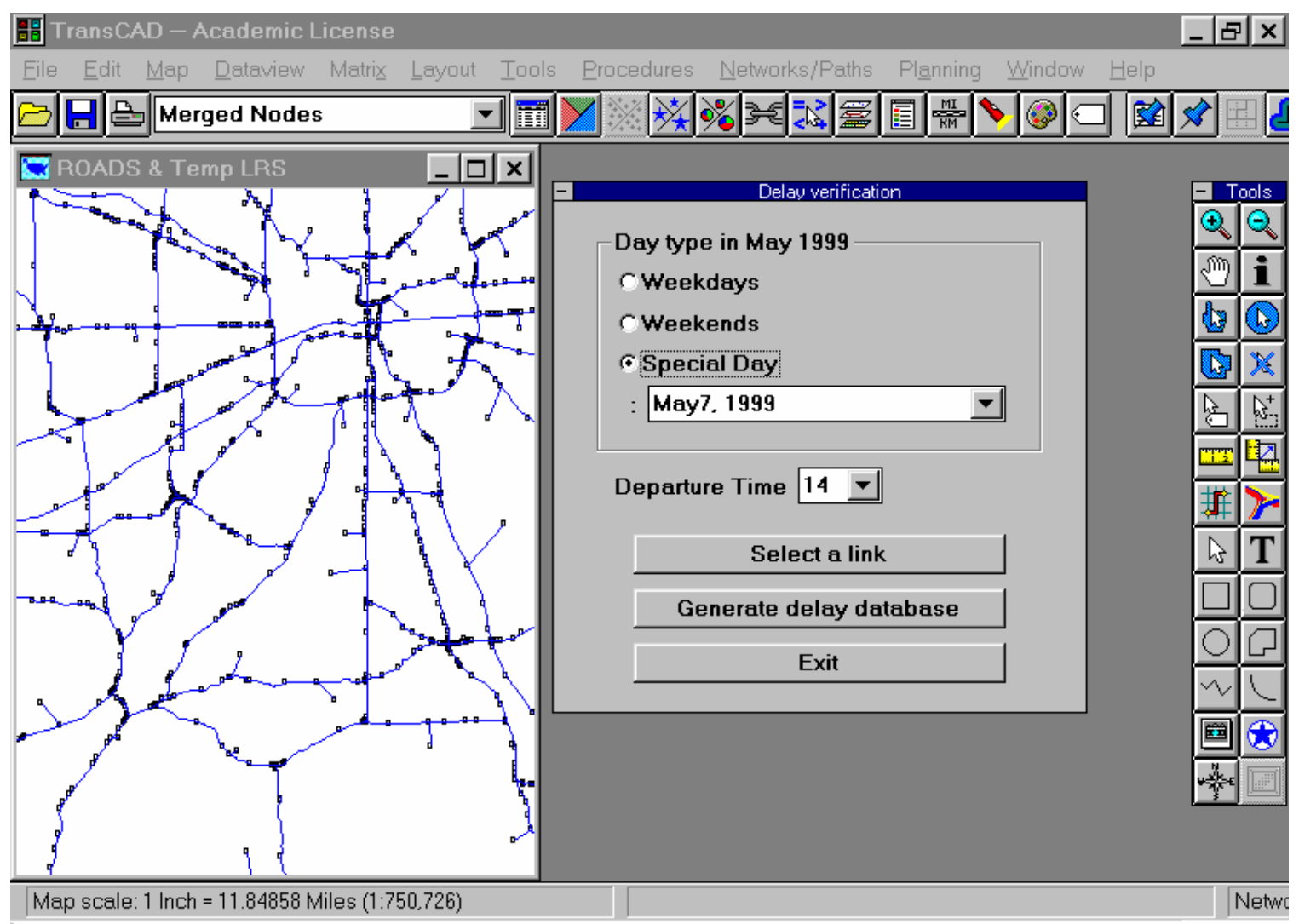

Figure A.18 Main menu for delay verification for TransCAD GISDK interface in modeling subsystem 


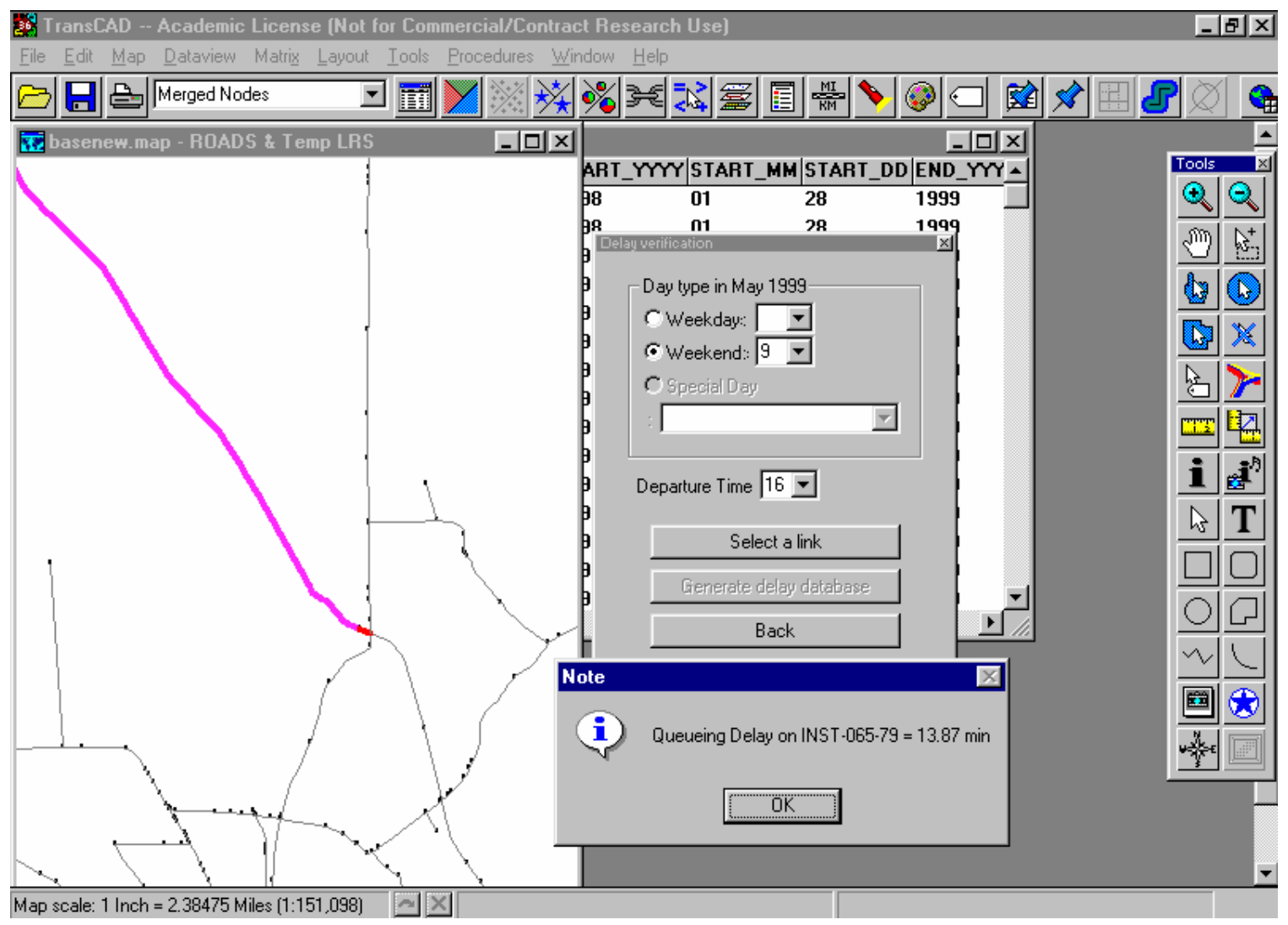

Figure A.19 Output of delay verification for upper bound analysis only 


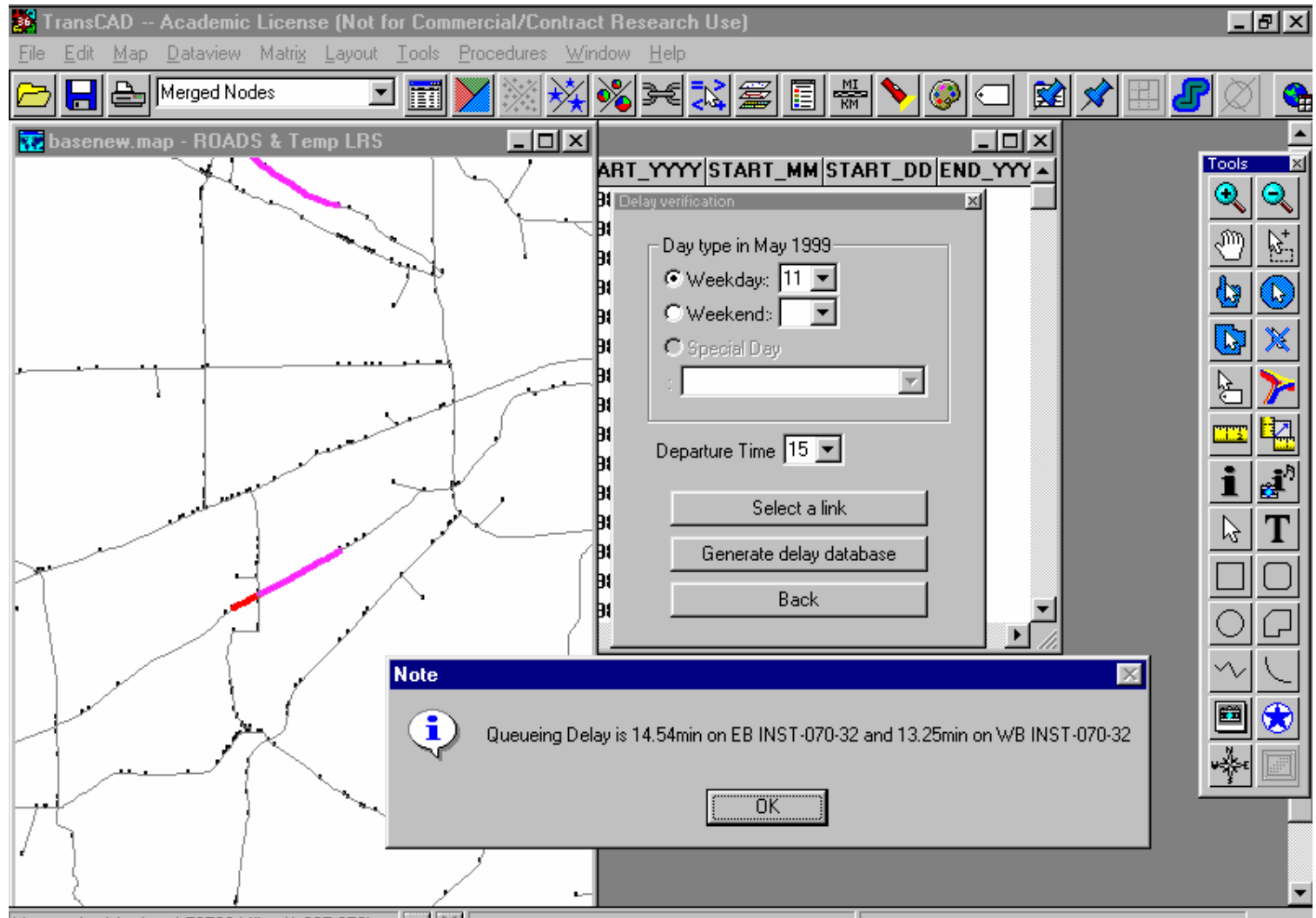

Map scale: 1 Inch $=4.53566$ Miles $(1: 287,379)$ X

Figure A.20 The output of delay verification for both upper bound and lower bound analyses 


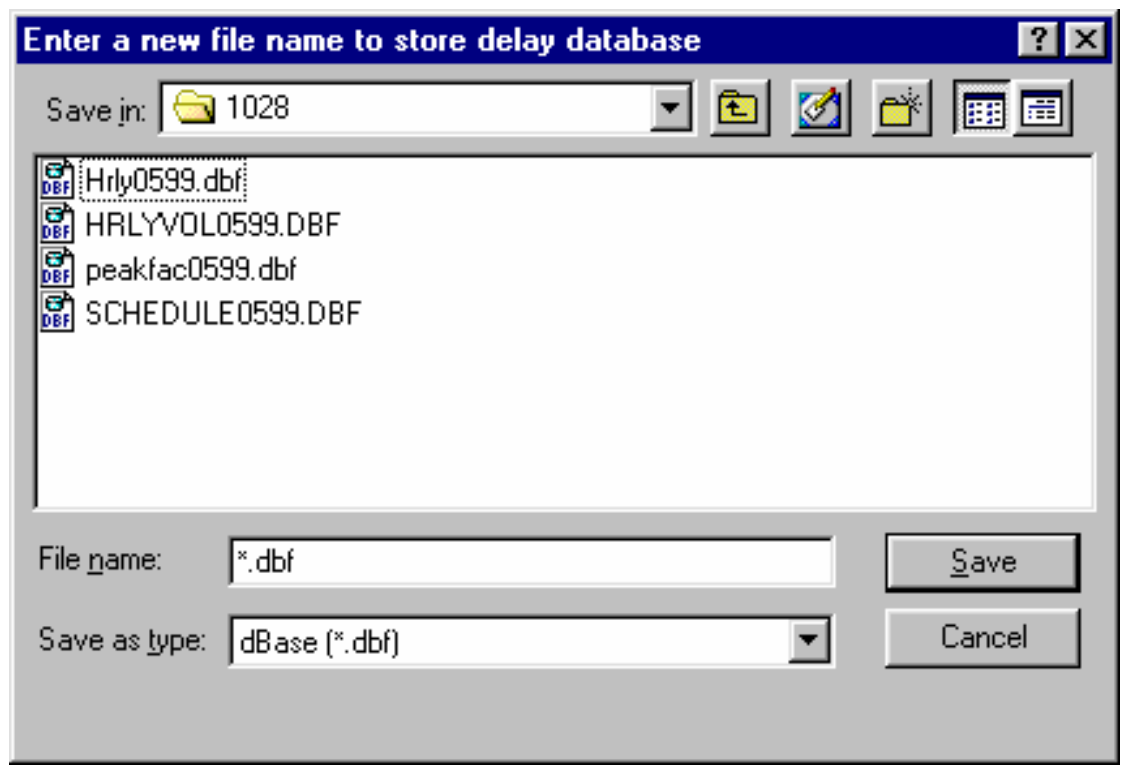

Figure A.21 The dialog box for inputting the file name for delay database 


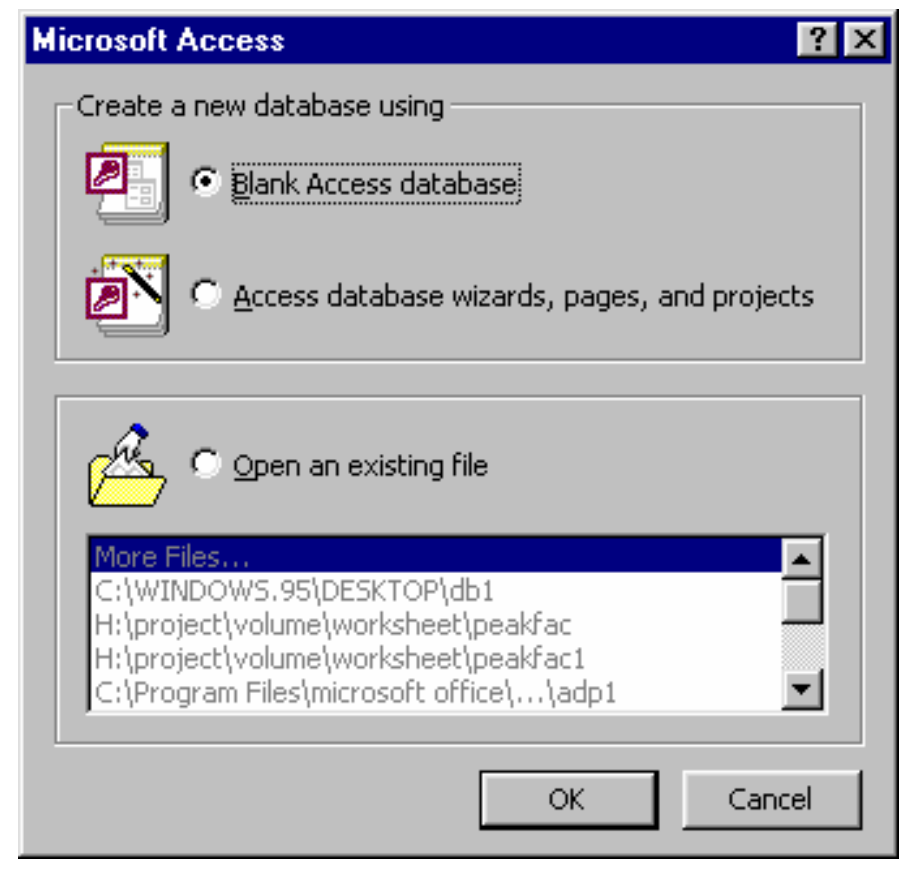

Figure A.22 Dialog box for creating a new Access database 


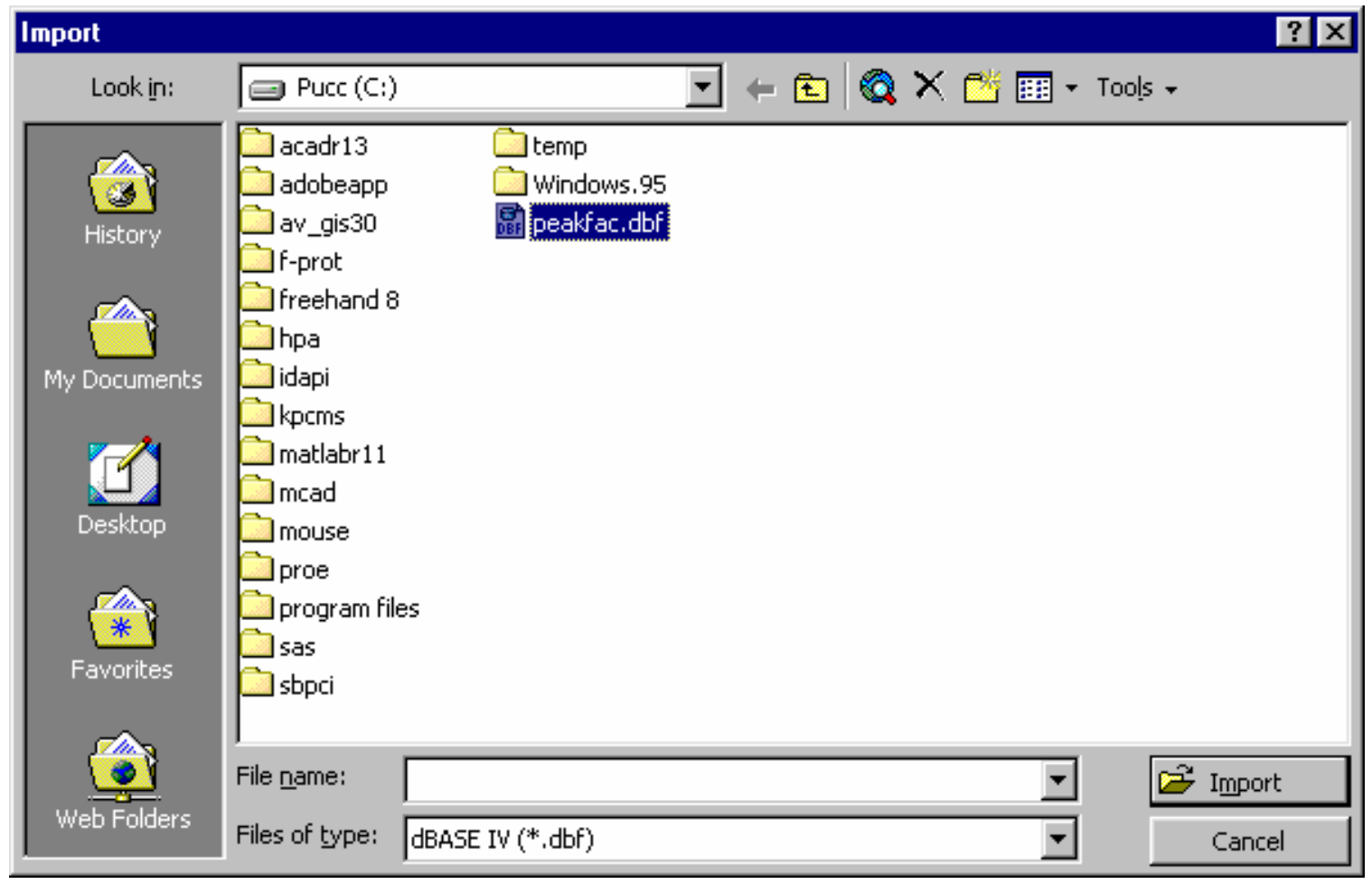

Figure A.23 Dialog box for importing a dBaseIV database 
Microsoft Access - [TEST0599 : Table]

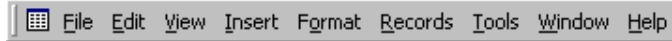

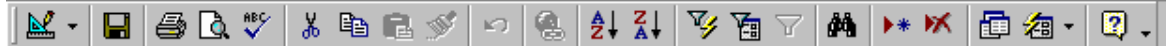

\begin{tabular}{|c|c|c|c|c|c|c|c|c|}
\hline PROJECT & TIME & NAME & AB_DELAY & BA_DELAY & LON & LAT & END_YYYY & $\Delta$ \\
\hline R23500 & 051513 & INST-065-79 & 0 & 0 & 158 & 194 & 1999 & \\
\hline R23500 & 051514 & INST-065-79 & 2.77 & 2.77 & 158 & 194 & 1999 & \\
\hline R23500 & 051515 & INST-065-79 & 8.32 & 8.32 & 158 & 194 & 1999 & \\
\hline R23500 & 051516 & INST-065-79 & 13.87 & 13.87 & 158 & 194 & 1999 & \\
\hline R23500 & 051517 & INST-065-79 & 5.26 & 5.26 & 158 & 194 & 1999 & \\
\hline R23500 & 051518 & INST-065-79 & 0 & 0 & 158 & 194 & 1999 & \\
\hline R23500 & 051519 & INST-065-79 & 0 & 0 & 158 & 194 & 1999 & \\
\hline R23500 & 051520 & INST-065-79 & 0 & 0 & 158 & 194 & 1999 & \\
\hline R23500 & 051521 & INST-065-79 & 0 & 0 & 158 & 194 & 1999 & \\
\hline R23500 & 051522 & INST-065-79 & 0 & 0 & 158 & 194 & 1999 & -1 \\
\hline R23500 & 051523 & INST-065-79 & 0 & 0 & 158 & 194 & 1999 & \\
\hline R23500 & 051600 & INST-065-79 & 0 & 0 & 158 & 194 & 1999 & \\
\hline R23500 & 051601 & INST-065-79 & 0 & 0 & 158 & 194 & 1999 & \\
\hline R23500 & 051602 & INST-065-79 & 0 & 0 & 158 & 194 & 1999 & \\
\hline R23500 & 051603 & INST-065-79 & 0 & 0 & 158 & 194 & 1999 & \\
\hline R23500 & 051604 & INST-065-79 & 0 & 0 & 158 & 194 & 1999 & \\
\hline R23500 & 051605 & INST-065-79 & 0 & 0 & 158 & 194 & 1999 & \\
\hline R23500 & 051606 & INST-065-79 & 0 & 0 & 158 & 194 & 1999 & \\
\hline R23500 & 051607 & INST-065-79 & 0 & 0 & 158 & 194 & 1999 & \\
\hline R23500 & 051608 & INST-065-79 & 0 & 0 & 158 & 194 & 1999 & \\
\hline R23500 & 051609 & INST-065-79 & 0.12 & 0.12 & 158 & 194 & 1999 & \\
\hline R23500 & 051610 & INST-065-79 & 0.35 & 0.35 & 158 & 194 & 1999 & \\
\hline R23500 & 051611 & INST-065-79 & 0.58 & 0.58 & 158 & 194 & 1999 & \\
\hline R23500 & 051612 & INST-065-79 & 0.01 & 0.01 & 158 & 194 & 1999 & \\
\hline R23500 & 051613 & INST-065-79 & 0 & 0 & 158 & 194 & 1999 & 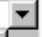 \\
\hline Record: $14|4| \begin{array}{ll} \\
\end{array}$ & $1 \mapsto \mid$ & of 10416 & 1 & & & & 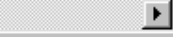 & \\
\hline \multicolumn{5}{|l|}{ Datasheet View } & & & NUM & \\
\hline
\end{tabular}

Figure A.24 An example delay database 


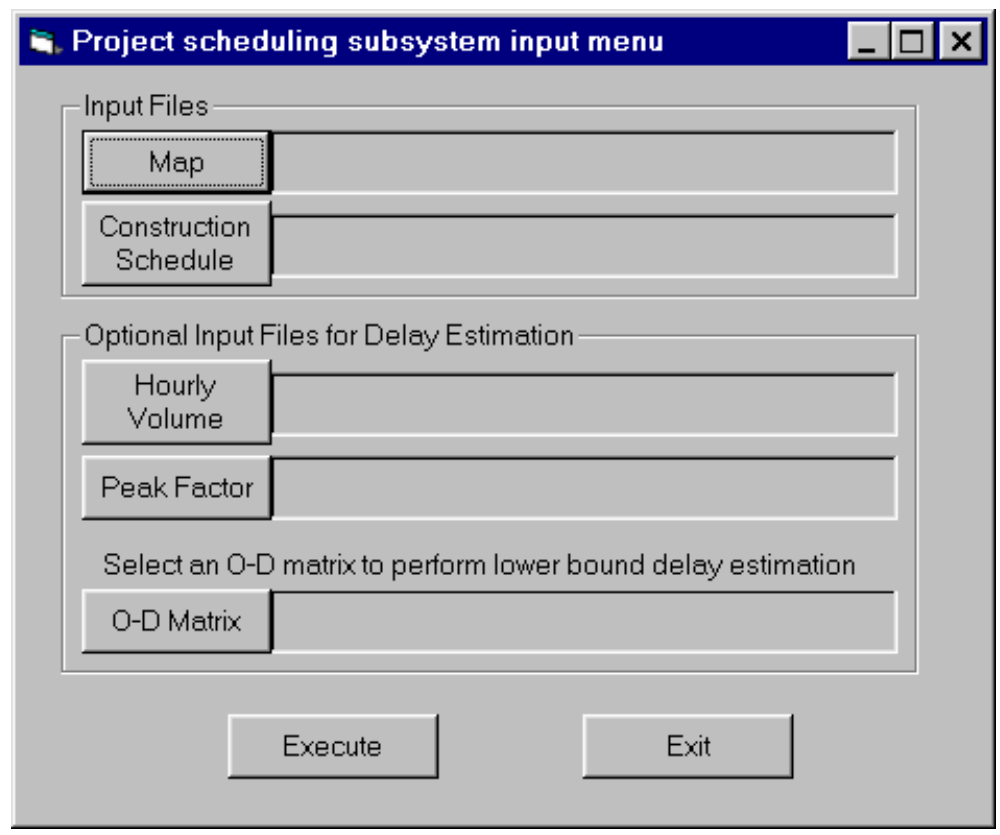

Figure A.25 Input menu for TransCAD GISDK interface in project scheduling subsystem 


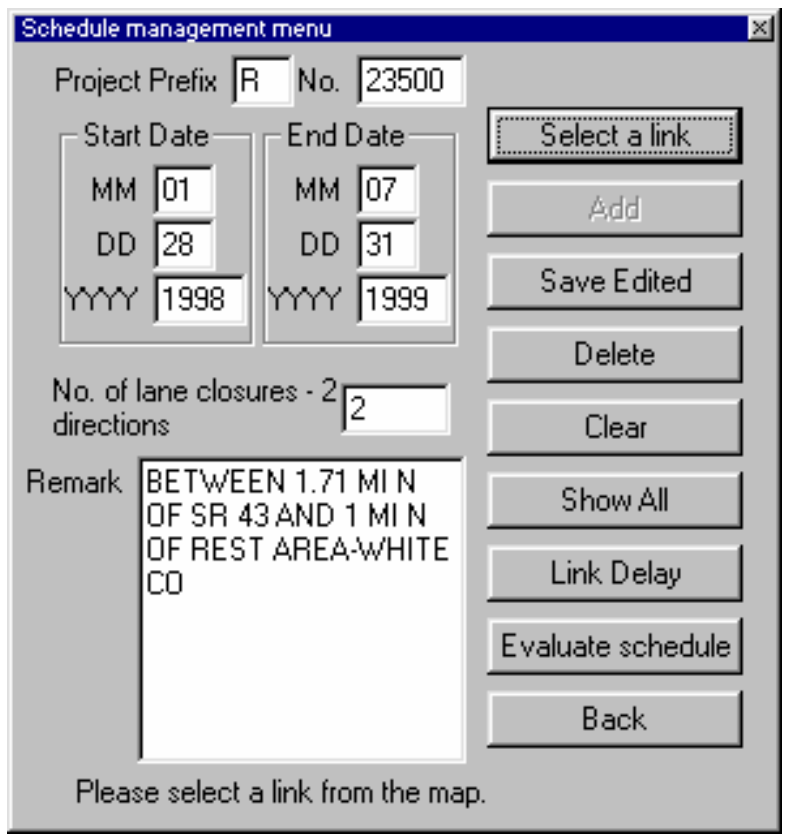

Figure A.26 Schedule management menu for TransCAD GISDK interface in project scheduling subsystem 


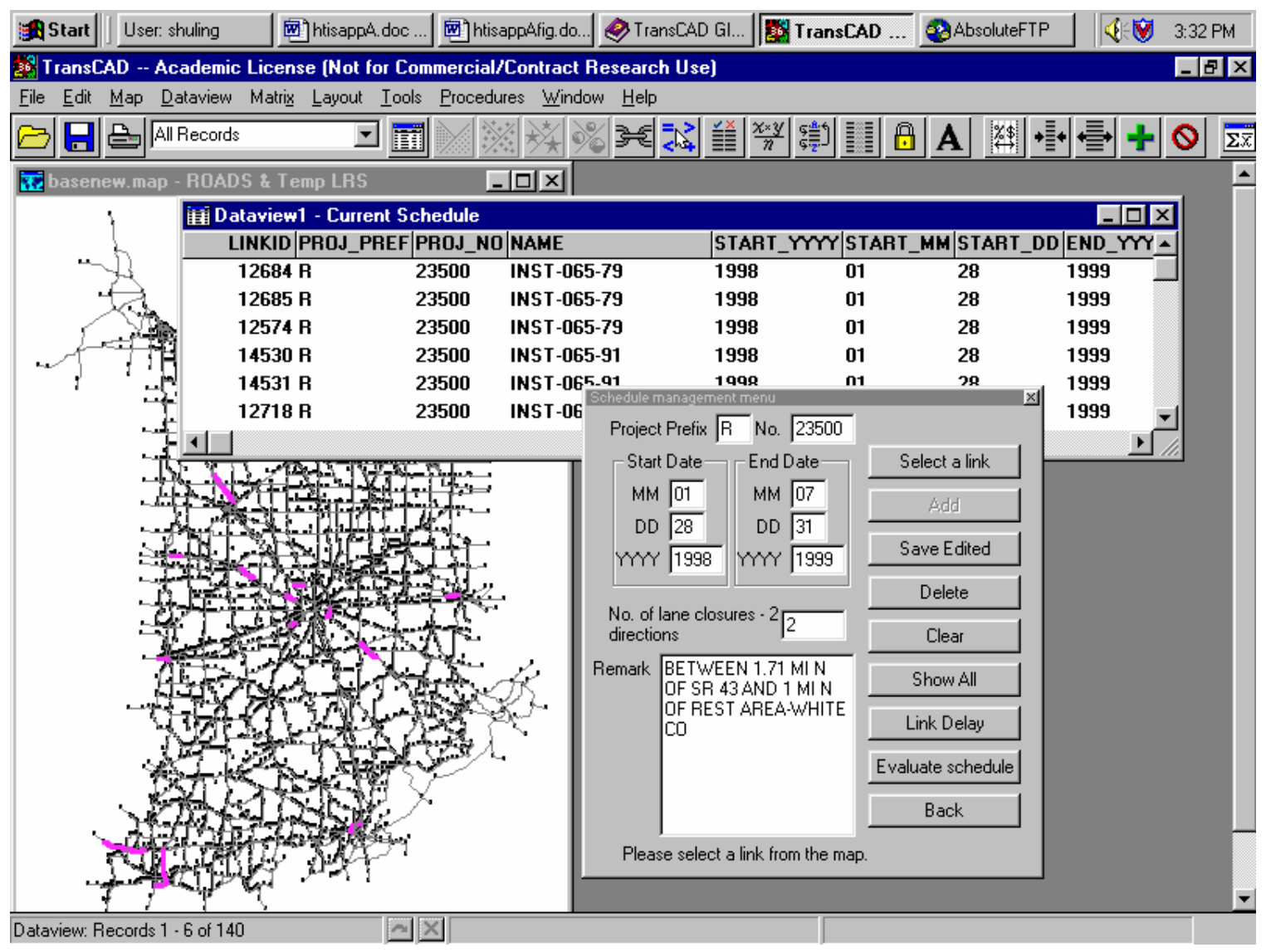

Figure A.27 The existing records in the scheduling database are shown in a new table and on the map 


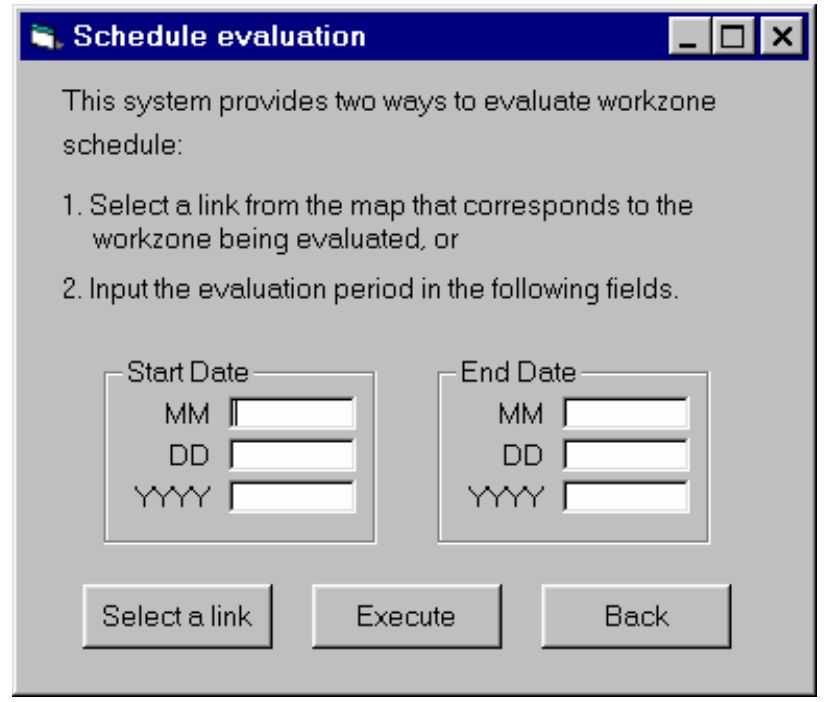

Figure A.28 Scheduling evaluation menu in project scheduling subsystem 


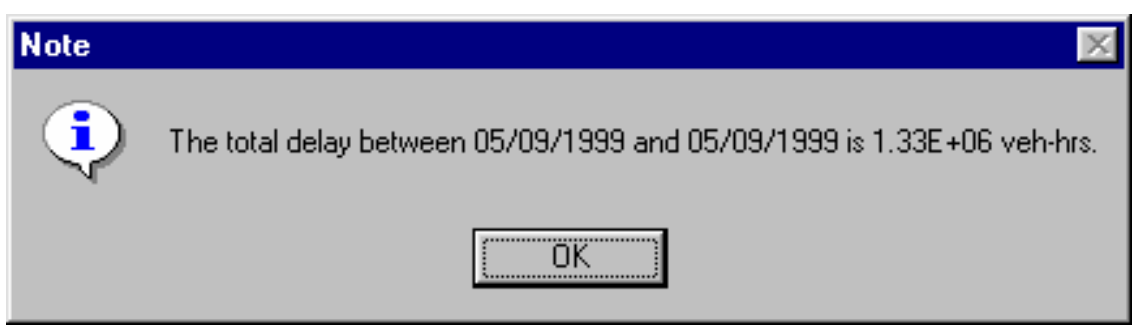

Figure A.29 The total delay of a specific period of time 


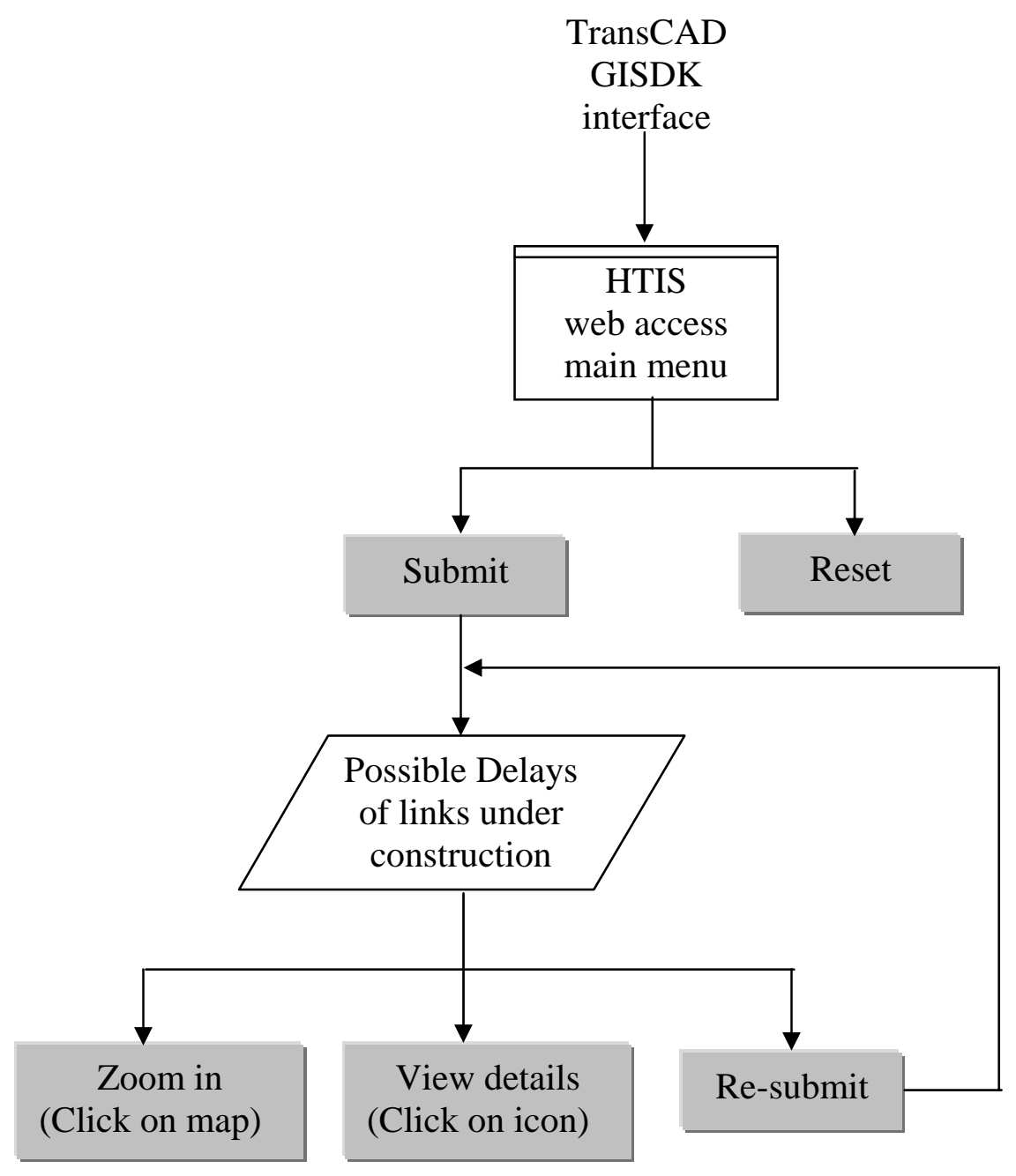

Figure A.30 Framework of the web access interface 


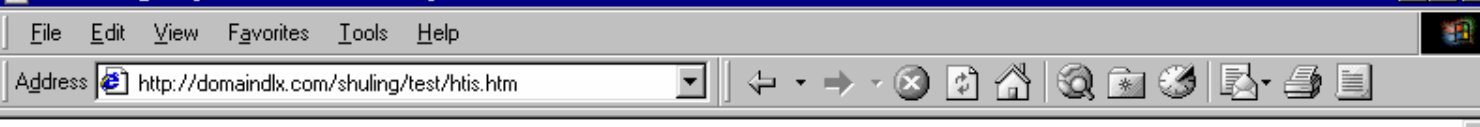

\section{Indiana Highway Travel Information System}

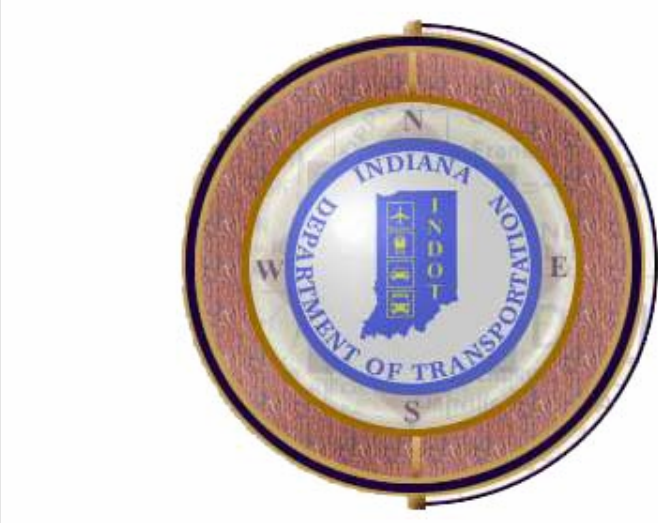

The Indiana Highway Travel Information System is offered to assist highway users in pre-trip planning. This system provides travel information on possible delays at workzones for both the remainder of the current month and the entire next month. After you select a desired departure date and time below and press "Submit", the system will provide an estimate of delays at highway construction zones. The estimated delays are based on expected traffic volumes for the date and time you choose. Because traffic patterns can vary, the delay estimates should be treated as approximations.

Departure Date: $08-24-2000($ Thu)

Departure Hour: 12:00AM 01:00AM

Submit Reset

Figure A.31 User's interface for travel information access subsystem 


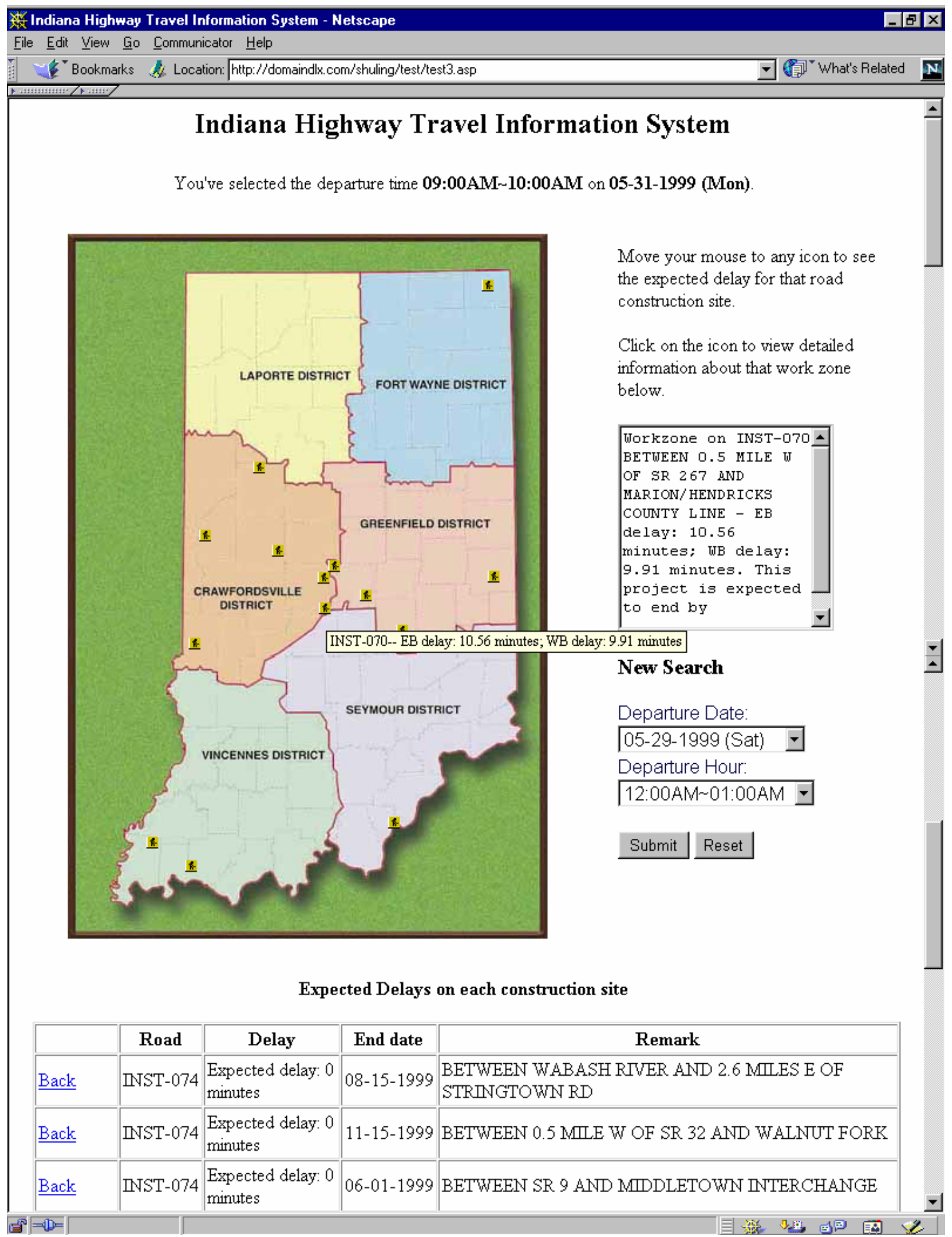

Figure A.32 Output screen for travel information access subsystem 